\title{
Characterization and Application of Bioengineered Heart Muscle as a New Tool to Study Human Heart Development and Disease
}

\author{
Doctoral Thesis \\ In partial fulfillment of the requirements for the degree \\ "Doctor rerum naturalium (Dr. rer. nat.)" \\ in the Molecular Medicine Study Program at the \\ Georg-August University Göttingen

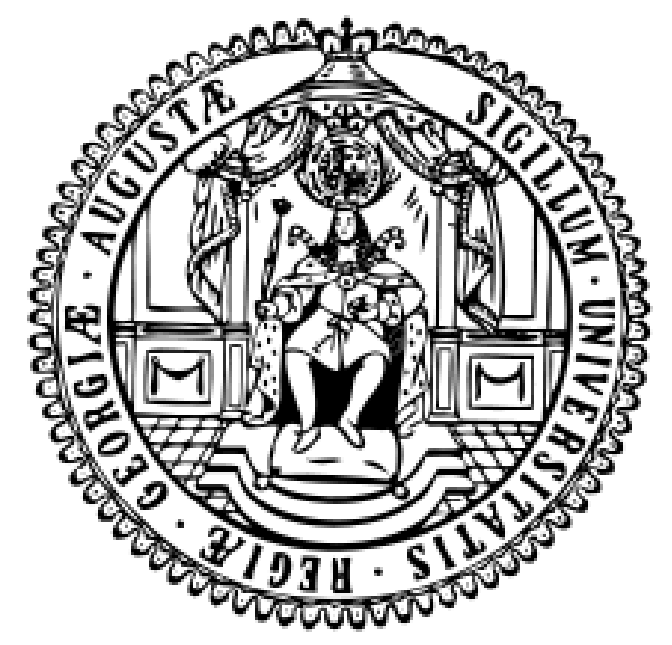

Submitted By:

Farah Safi Raad

Born in Beirut, Lebanon

Göttingen, 2016 


\title{
Thesis Committee Members
}

\section{Prof. Dr. med. Wolfram, H- Zimmermann (Supervisor)}

Email: W.zimmermann@med.uni-goettingen.de

Phone: + +49551395787

Postal Address: $\quad$ Institute of Pharmacology and Toxicology

University Medical Center Göttingen

Georg-August University Göttingen

Robert-Koch-Str. 40

37075 Götttingen

Germany

Prof. Dr. Dr. h.c. Lutz-F. Tietze (First thesis committee member)
Email: $\quad$ ltietze@gwdg.de
Phone: $\quad$ +495513933271/-72
Postal Address: $\quad$ Institute of Organic and Biomolecular Chemistry
Georg-August University Göttingen
Tammannstr. 2
37077 Göttingen
Germany

\author{
Prof. Dr. Andreas Wodarz (Second thesis committee member) \\ Email: $\quad$ andreas.wodarz@uk-koeln.de \\ Phone: $\quad$ +4922147882880 \\ Postal Address: $\quad$ Institute of Anatomy \\ University of Cologne \\ Kerpener Str. 62 \\ 50397 Cologne \\ Germany
}




\section{Affidavit}

Here I declare that my doctoral thesis entitled

"Characterization and Application of Bioengineered Heart Muscle as a New Tool to Study Human Heart Development and Disease”

has been written independently with no other sources and aids than quoted.

Raad, Farah

Göttingen, May 2016 


\section{List of Publications}

1) Zeidler S, Meckbach C, Tacke R, Raad FS, Roa A, Uchida S, Zimmermann WH, Wingender E and Gueltas M (2016). Computational Detection of Stage-Specific Transcription Factor Clusters during Heart Development. Front. Genet. 7:33

2) Tiburcy M, Hudson JE, Balfanz P, Schlick S, Meyer T, Chang Liao ML, Levent E, Raad FS, Zeidler S, Wingender E, Riegler S, Wang M, Gold JD, Kehat I, Wettwer E, Ravens U, Dierickx P, Van Laake L, Goumans MJ, Toischer K, Hasenfuss G, Couture LA, Unger A, Linke W, Araki T, Neel B, Keller G, Gepstein L, Wu J and Zimmerman WH. (2016). Directed Maturation of Engineered Human Myocardium for Applications in Heart Failure Modelling and Repair. In Revision.

3) Hudson JE, Tiburcy M, Raad FS, Roa A, Chang Liao ML, Muppala V, Doeker S, Uchida S, John D, Ortmann D, Pedersen RA, Elliot DA, Moebius W, Bartels I, Dressel R, Ziebolz D, Behr R and Zimmermann WH. (2016). Directed Assembly of Human and Non-Human Primate Heart Muscle from Embryonic and Induced Pluripotent Stem Cells. In Revision.

\section{List of Posters and Presentations}

1) Farah S Raad, Angelica Roa, James E Hudson and Wolfram H Zimmermann. (2014). Altering Tissue Functionality using Growth Factors and Small Molecules in a Bioengineered Heart Muscle. DGK Basic Science Meeting 2014, Duesseldorf, Germany. (Poster).

2) Farah S Raad, Malte Tiburcy, Angelic Roa and Wolfram H Zimmermann. (2015). Simulating Human Myocardium in vitro. Fondation Leducq Meeting 2015, Trieste, Italy. (Power point Presentation). 
3) Farah S Raad, Angelica Roa, James E Hudson, Malte Tiburcy, Shizuka Uchida and Wolfram H Zimmermann. (2016). Modeling Human Heart Development and Faulty Cardiogenesis in Bioengineered Heart Muscle. DGK-HKK Mannheim 2016. Mannheim, Germany. (Poster). 


\section{Table of Contents}

Thesis Committee Members ............................................................................................................................

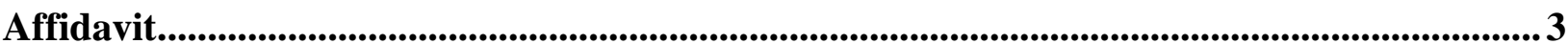

List of Publications................................................................................................................................................. 4

List of Posters and Presentations ................................................................................................... 4

Table of Contents .................................................................................................................................................. 6

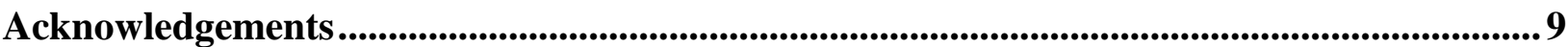

Abstract ............................................................................................................................................................. 10

List of Figures ............................................................................................................................................ 11

List of Tables........................................................................................................................................................... 13

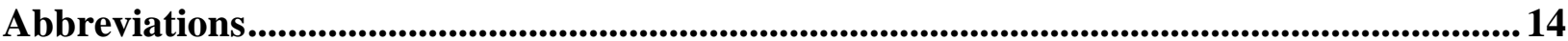

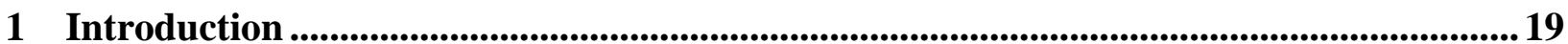

1.1 Embryonic Heart Development......................................................................................... 19

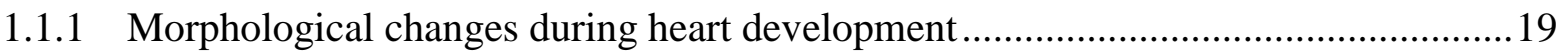

1.1.2 Molecular changes during heart development .........................................................22

1.2 Directed Cardiac Differentiation of Stem Cells ................................................................2 24

1.3 Tissue Engineering: Basis for Modeling Heart Development in a Dish ...................... 28

1.4 Bioengineered Heart Muscle: Concept and Preliminary Data...................................... 31

1.5 Bone Morphogenetic Protein Signal Transduction ........................................................ 33

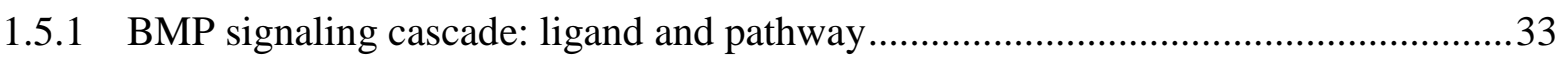

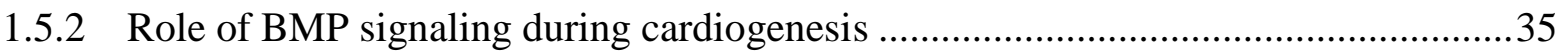

1.5.3 Chalcones: potential candidate for BMP4 replacement .............................................

1.6 Thesis Objectives and Hypotheses ............................................................................ 41

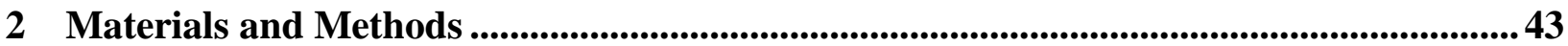

2.1 Cell Culture ......................................................................................................................... 43

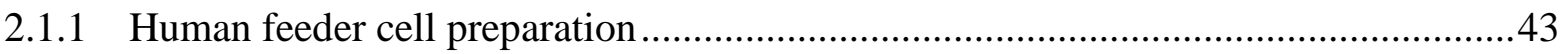

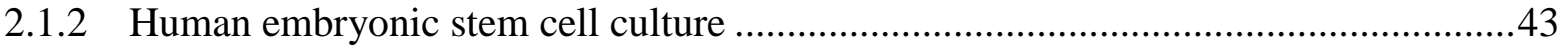

2.2 2D Cardiac Differentiation ..................................................................................4 44

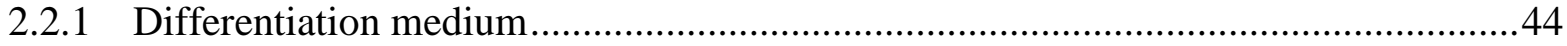

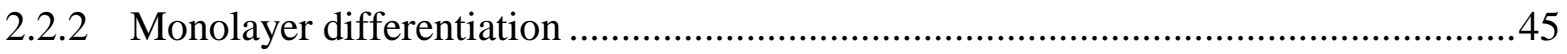

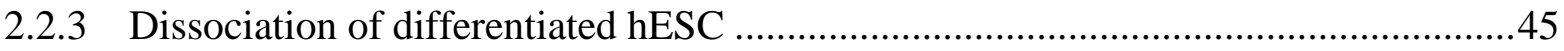

2.3 Bioengineered Heart Muscle Generation ............................................................................... 45 
2.3.1 Preparation of casting molds and dynamic stretchers ........................................45

2.3.2 Bioengineered heart muscle assembly and differentiation ......................................46

2.3.3 Bioengineered heart muscle dissociation ..............................................................4 47

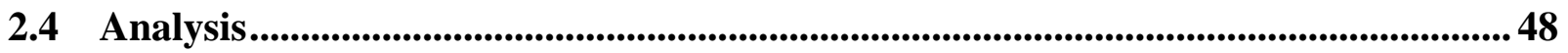

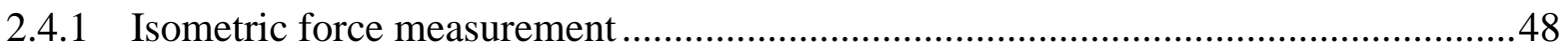

2.4.2 Action potential recordings ......................................................................... 48

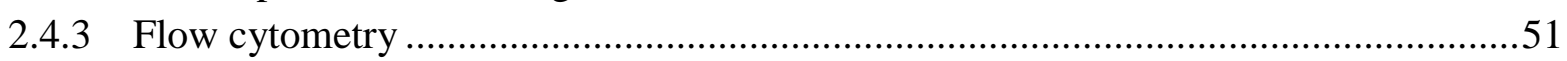

2.4.4 Quantification of cardiomyocyte size using flow cytometry ................................52

2.4.5 Whole mount staining and confocal imaging ..................................................52

2.4.6 Transmission electron microscopy ......................................................................52

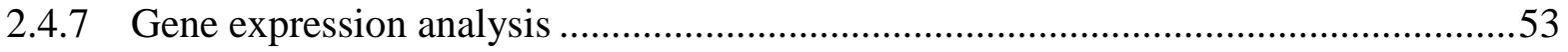

2.4.8 RNA sequencing and bioinformatics analyses.................................................54

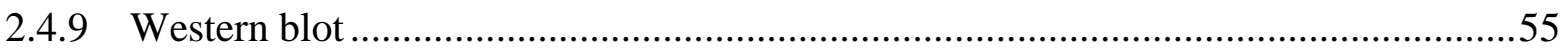

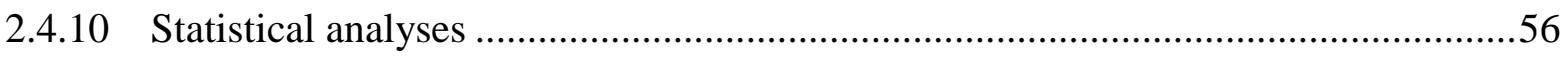

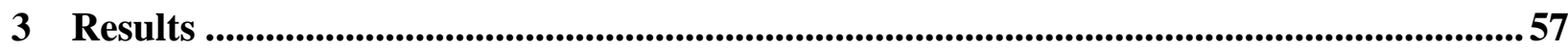

3.1 Characterization of a Bioengineered Heart Muscle ..................................................5 57

3.1.1 Directed self-assembly of bioengineered heart muscle...........................................57

3.1.2 Gene expression profiling of developing bioengineered heart muscle ......................57

3.1.3 Cell composition of bioengineered heart muscle ...................................................59

3.1.4 Functional properties of bioengineered heart muscle ...............................................61

3.1.5 Continuous maturation of bioengineered heart muscle by day 60 ..........................64

3.1.6 Evidence for cardiac-neuro crosstalk during BHM development..............................70

3.1.7 Applications of the BHM model in disease modeling and drug screening................75

3.2 Enhancing Maturation of Bioengineered Heart Muscle .......................................... 79

3.2.1 Variation of TGF $\beta-1$ stimulation during cardiac maturation ..................................79

3.2.2 Omission of insulin during cardiac maturation.................................................... 83

3.2.3 Optimization of basal medium in long-term cultures ........................................85

3.3 Replacement of BMP4 by a Small Molecule Mimic during Mesoderm Induction... 86

3.3.1 Re-evaluation of the role of BMP4 for mesoderm induction in monolayer culture ...86

3.3.2 Re-evaluation of the role of BMP4 for mesoderm induction in BHM ......................88

3.3.3 Effect of BMP4 on gene expression profiles during BHM development ..................89

3.3.4 Chalcones as potential small molecule activators of BMP signaling .......................90

3.3.5 Formation of bioengineered heart muscle with BMP mimics ...............................94

4 Discussion .........................................................................................................................96

4.1 Simulation of Human Heart Development in Bioengineered Heart Muscle ............. 98

4.2 Evidence for Functional Neuro-crest Contribution in Bioengineered Heart Muscle102

4.3 Modeling of Faulty Cardiogenesis Induced by BMP Inhibition ................................ 103

4.4 Proof-of-concept for the Application of BHM in Drug Screening ............................ 104 
4.5 Enhanced Functional Maturation of Bioengineered Heart Muscle ......................... 105

4.6 Stimulation of Mesoderm Induction with a Small Molecule BMP Mimic ............... 107

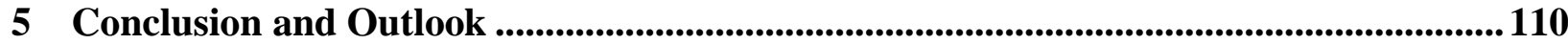

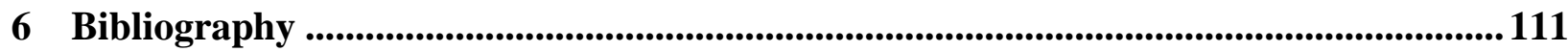

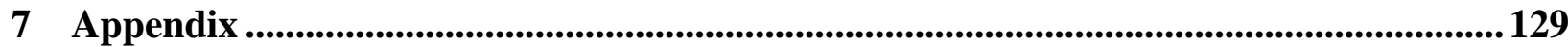




\section{Acknowledgements}

I became part of Prof. Wolfram H. Zimmermann's group in 2011 and ever since then the people I have encountered and got to know became part of me. For that, I am thankful for this experience which helped me both at the personal and intellectual level.

First I would like to express my sincere gratitude to my advisor Prof. Dr. Wolfram H. Zimmermann for the continuous support of my $\mathrm{PhD}$ study and related research, for his patience, motivation and immense knowledge. His guidance helped me in all the time of research and writing of this thesis. Besides my advisor, I would like to thank the rest of my thesis committee: Prof. Dr. Dr. h.c. Lutz F. Tietze and Prof. Andreas Wodarz for their insightful comments and encouragement, but also for the hard question which incented me to widen my research from various perspectives. I would also like to thank Prof. Susanne Lutz, Prof. Thomas Meyer and Dr. Katrin Streckfuß-Bömeke for the willingness to be my examination referees.

My sincere thanks go to Dr. Angelica Roa, Dr. James E. Hudson, Dr. Maria-Patapia Zafeiriou and Dr. Laura Zelarayan-Behrend for their constant guidance, support and advice related to science as well as personal matters.

I am very grateful to have met wonderful people from different parts of the world which taught me a lot about life and culture. These same people are not only my friends but also like a family to me in the good and bad times. I would like to thank Dr. Poh Long Soong, Dr. Norman Liaw, Dr. Buntaro Fujita, Dr. Mei-Ling Liao, Elif Levent, Eriona Heta, Monique Wölfer and Satish Galla. Thank you for being there.

A huge gratitude goes to my family back in Lebanon especially to my mom and my future husband Mohamad Fakih for their continuous support and encouragement to achieve the best in life. Last but not least, I am very grateful to have my sister Dr. Nour Raad beside me in all situations. I do not know what would have I done without her. 


\section{Abstract}

Proof-of-concept for the generation of a bioengineered heart muscle (BHM) directly from human pluripotent stem cells (hPSCs) as a model to simulate human heart muscle development was introduced recently by our lab. BHMs undergo stage-specific directed differentiation and self-assembly guided by the sequential addition of growth factors and small molecules to support (1) mesoderm induction (3 days), (2) cardiac specification (10 days) and (3) cardiac maturation (up to 50 days studied). By culture day 22, initial findings revealed homogeneously contracting BHMs with robust inotropic responses to increasing extracellular calcium and $\beta$ adrenergic stimulation. In this thesis, a detailed characterization on the molecular, cellular and functional level revealed that BHMs (1) do indeed traverse through defined in-utero like developmental stages with characteristic transcriptome profiles, (2) are composed of mainly mesodermal cells (cardiomyocytes and fibroblast-like cells), (3) display continuous functional maturation over time with enhanced contractile performance on the cellular level and (4) develop in late cultures (day 60) a functional neural crest component with resemblance to the cardiac sympathetic nervous system. Assessments of drug responses revealed the utility of BHM in disease modeling and drug screening. Modulation of one of the pivotal signaling pathways implicated in early cardiac induction, such as the BMP pathway during BHM development revealed that low concentrations of BMP4 are needed for the optimal differentiation of hPSCs to both cardiomyocytes and fibroblast-like cells. In a first effort to reduce all protein stimuli from the BHM culture format, screening for BMP4 replacements was performed and identified two hit molecules (4'-hydroxychalcone and 4-fluoro-4'-methoxychalcone). Both successfully induced cardiac differentiation in monolayer culture when added as BMP4 replacements, but not in the BHM culture format. Collectively, this is the first detailed characterization of a novel cardiac organoid model (BHM), generated by a single step tissue engineering approach directly from hPSCs, with organotypic contractile functionality. Applications in drug screening and disease modeling are demonstrated. Further improvements may be achieved by the replacement of all protein culture medium supplements by bioactive small molecules, such as chalcones to replace BMP4. 


\section{List of Figures}

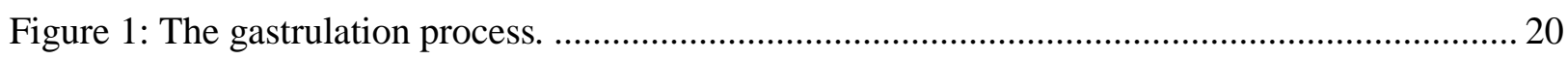

Figure 2: Overview of the formation of the four-chambered heart................................................. 22

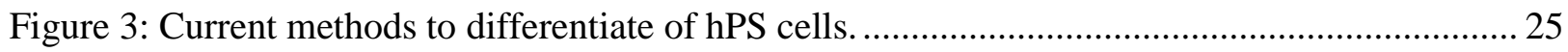

Figure 4: Overview of tissue engineering approaches, cell sources and contractility evaluation. ..... 29

Figure 5: Morphological and functional assessment of BHM at day 22........................................... 32

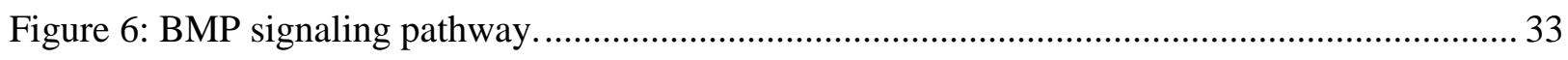

Figure 7: Signaling pathways and gene regulators governing cardiomyogenesis............................... 36

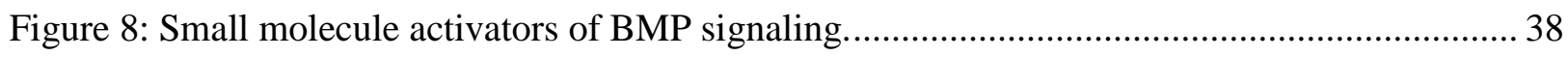

Figure 9: Backbone of the chalcone family and examples of biological activities. .............................4 40

Figure 10: Schematic overview of the work program. ………..................................................... 42

Figure 11: Outline of the cardiac differentiation of HES2 cells in monolayer cultures...................... 44

Figure 12: Casting mold and silicone poles for BHM generation and loading. .................................. 46

Figure 13: Outline of HES2-BHM generation and differentiation.................................................. 47

Figure 14: Action potential traces and membrance currents from ventricular and pacemaker cells.. 50

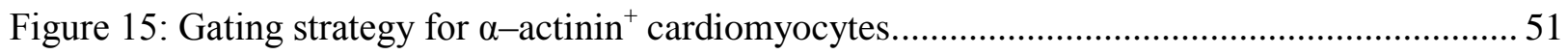

Figure 16: Molecular profiles during BHM development.............................................................. 58

Figure 17: Changes of cell number and composition of BHM. ......................................................... 60

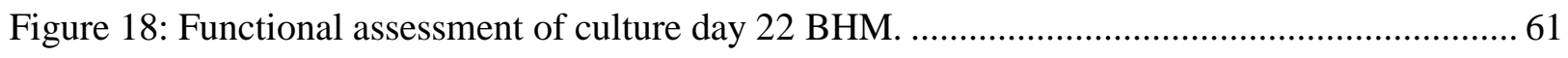

Figure 19: Electrophysiological properties of cardiomyocytes in BHM at culture day 22.................62 62

Figure 20: Expression profile for cardiac ion channels and calcium homeostasis associated genes. 63

Figure 21: Functional, morphological and cell count analyses of BHMs in long-term cultures......... 65

Figure 22: Further functional and morphological maturation in long-term BHM cultures................. 66

Figure 23: Correlation of cardiomyocyte content and cardiac actin (ACTC1) in BHM......................67 67

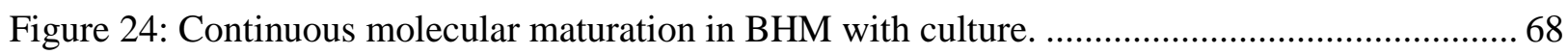

Figure 25: Enhanced cardiac ion channel and calcium homeostasis associated gene expression...... 69

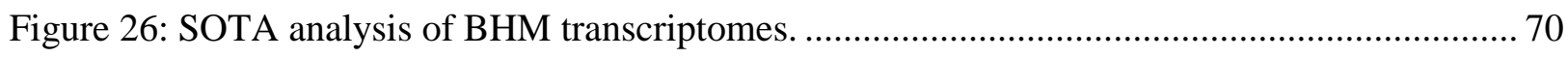

Figure 27: Molecular and morphological evidence for neural crest development in BHM................ 72

Figure 28: Evidence for a sympathetic component in day 60 BHM. ................................................. 74

Figure 29: Identification of faulty cardiomyogenesis in BHM under BMP inhibition...................... 76 
Figure 30: Identification of the biological activity of reference compounds in BHM.................... 78

Figure 31: Functional maturation of BHMs by transient TGF $\beta-1$ stimulation. ............................... 81

Figure 32: Cross sectional area of BHM under TGF $\beta-1$ stimulation. ........................................... 82

Figure 33: Stabilization of long-term outcome by transient TGF $\beta-1$ supplementation. .................. 83

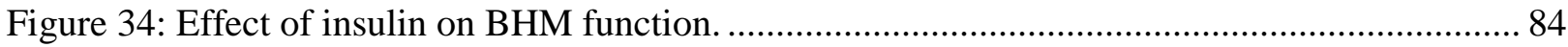

Figure 35: Consequences of basal medium on BHM function and cellularity............................... 85

Figure 36: Effects of BMP4 on mesoderm induction in monolayer cultures.................................. 87

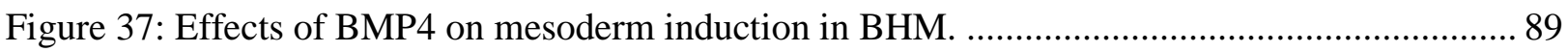

Figure 39: Preliminary screen of chalcones as BMP4 mimics for mesoderm induction. ................. 92

Figure 40: Small scale screen of chalcones as BMP4 mimics during mesoderm induction. ............. 93

Figure 41: SMAD and ERK phosphorylation in response to BMP4 ......................................... 94

Figure 42: Schematic outline of the optimized long-term BHM culture/maturation protocol......... 107 


\section{List of Tables}

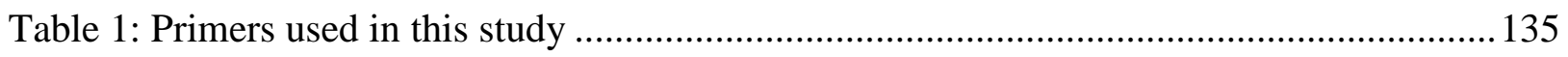

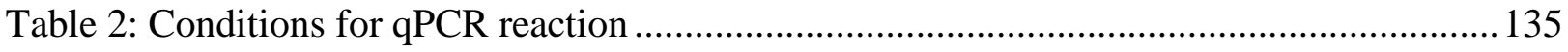

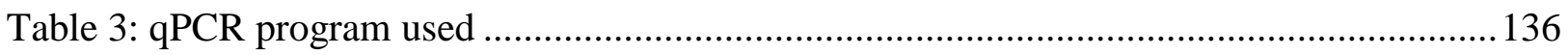

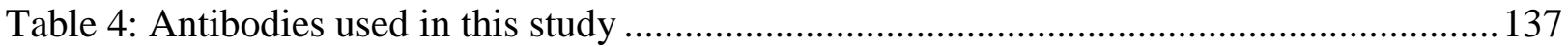




\begin{tabular}{|c|c|}
\hline \multicolumn{2}{|c|}{ Abbreviations } \\
\hline$\overline{\alpha-M E M}$ & $\alpha$-Minimum Essential Medium \\
\hline$\alpha$-SMA & $\alpha$-Smooth Muscle Actin \\
\hline$\alpha-\mathrm{MHC}$ & $\alpha$-Myosin Heavy Chain \\
\hline ACTN2 & Actinin alpha 2 \\
\hline ACTC1 & Actin alpha Cardiac Muscle 1 \\
\hline ALDH1A2 & Aldehyde Dehydrogenase 1 Family Member A2 \\
\hline ATP2A2 & ATPase Sarcoplasmic/Endoplasmic Reticulum Calcium Transporting 2 \\
\hline ALK2 & Activin A Receptor Type 1 \\
\hline ActR-II & Activin Receptor Type II \\
\hline Asc-2P & Ascorbic Acid 2-Phosphate \\
\hline AP & Action Potential \\
\hline APD & Action Potential Duration \\
\hline APA & Action Potential Amplitude \\
\hline $\mathrm{Akt} / \mathrm{PKB}$ & Akt Protein Kinase B \\
\hline BMP & Bone Morphogenetic Protein \\
\hline BHM & Bioengineered Heart Muscle \\
\hline BMPR(I/II) & Bone Morphogenetic Protein Receptor type I/II \\
\hline bHLH & Basic Helix-Loop-Helix \\
\hline BAMBI & BMP and Activin Membrane-Bound Inhibitor \\
\hline BSA & Bovine Serum Albumin \\
\hline BDM & 2,3-Butanedione Monoxime \\
\hline $\mathrm{Cx} 40$ & Connexin 40 \\
\hline $\mathrm{Cx} 43$ & Connexin 43 \\
\hline cTNT & Cardiac Troponin $\mathrm{T}$ \\
\hline CD90 & Thy-1 Cell Surface Antigen \\
\hline CD117 & KIT Proto-Oncogene Receptor Tyrosine Kinase \\
\hline CD45 & Leukocyte Common Antigen \\
\hline CD31 & Platelet/Endothelial Cell Adhesion Molecule \\
\hline CACNA1C & Calcium Voltage-gated Channel Subunit alpha1 C \\
\hline CASQ2 & Calsequestrin 2 \\
\hline
\end{tabular}




\begin{tabular}{|c|c|}
\hline cDNA & Complementary DNA \\
\hline Co-SMAD & Cooperating SMAD \\
\hline CHD & Congenital Heart Disease \\
\hline $\mathrm{CM}$ & Cardiomyocyte \\
\hline CBP & CREB- Binding Protein \\
\hline Ctrl & Control \\
\hline DDR & Diastolic Depolarization Rate \\
\hline DNA & Deoxyribonucleic Acid \\
\hline DKK1 & Dickkopf WNT signaling pathway inhibitor 1 \\
\hline DMH1 & Dorsomorphin Homologue 1 \\
\hline DMEM & Dulbecco's Modified Eagle Medium \\
\hline DEPC water & Diethylpyrocarbonate-treated Water \\
\hline EHM & Engineered Heart Muscle \\
\hline ERK1/2 & Extracellular Signal-regulated Kinase $1 / 2$ \\
\hline EB & Embryoid Body \\
\hline EDTA & Ethylenediaminetetraacetic Acid \\
\hline FOXA2 & Forkhead box A2 \\
\hline FGF & Fibroblast Growth Factor \\
\hline FYVE & Four Cysteine-rich Proteins: Fab1, YOTB, Vac 1 and EEA1 \\
\hline FHF & First Heart Field \\
\hline FBS & Fetal Bovine Serum \\
\hline FPKM & Fragments Per Kilobase of Exon per Million Fragments \\
\hline FOC & Force of Contractilition (systolic force amplitude) \\
\hline GATA4 & GATA Binding Protein 4 \\
\hline GDF & Growth Differentiation Factor \\
\hline GS domain & Glycine and Serine-rich Sequence Domain \\
\hline $\mathrm{GABA}_{\mathrm{B}}$ & Receptor for Gamma-aminobutyric Acid \\
\hline GAPDH & Glyceraldehyde-3-Phosphate-Dehydrogenase \\
\hline GO & Gene Ontology \\
\hline GSK3 $\beta$ & Glycogen Synthase Kinase $3 \beta$ \\
\hline hPSC & Human Pluripotent Stem Cells \\
\hline
\end{tabular}




$\begin{array}{ll}\text { hESC } & \text { Human Embryonic Stem Cells } \\ \text { hIPSC } & \text { Human Induced Pluripotent Stem Cells } \\ \text { HAND2 } & \text { Heart and Neural Crest Derivatives Expressed 2 } \\ \text { HCN4 } & \text { Hyperpolarization Activated Cyclic Neucleotide-gated Potassium Channel } 4 \\ \text { HFF } & \text { Human Foreskin Fibroblast } \\ \text { ISL1 } & \text { Islet 1 } \\ \text { IWR-1 } & \text { Inhibitor of Wnt Response-1 } \\ \text { Id1-3 } & \text { Inhibitor of Differentiation 1-3 } \\ \text { I-SMAD } & \text { Inhibitory- SMAD } \\ \text { IR-HFF } & \text { Irradiated HFF } \\ \text { IWP4 } & \text { Inhibitor of Wnt Production- 4 } \\ \text { IGF-1 } & \text { Insulin-like Growth Factor 1 } \\ \text { IgG } & \text { Immunoglobulin G } \\ \text { IMDM } & \text { Iscove's Modified Dulbecco's Medium } \\ \text { K } 2.1 & \text { Potassium Voltage Gated Channel } \\ \text { KOSR } & \text { Knock-Out Serum Replacement } \\ \text { KEGG } & \text { Kyoto Encyclopedia of Genes and Genomes } \\ \text { KCNH2 } & \text { Potassium Voltage-gated Channel Subfamily H Member 2 } \\ \text { MDP } & \text { Maximal Diastolic Potential } \\ \text { MESP1 } & \text { Mesoderm Posterior Basic Helix-Loop-Helix Transcription Factor 1 } \\ \text { MEF2C } & \text { MADS Box Transcription Enhancer Factor 2, Polypeptide C } \\ \text { MLC2a } & \text { Atrial Myosin Light Chain 2 } \\ \text { MLC2v } & \text { Ventricular Myosin Light Chain 2 } \\ \text { MH domain } & \text { Mad Homology Domain } \\ \text { MIXL1 } & \text { Mix Paired-Like Homeobox 1 } \\ \text { MYH6 } & \text { Myosin Heavy Chain 6 (gene encoding for } \alpha-\text { MHC protein) } \\ \text { MYH7 } & \text { Myosin Heavy Chain 7 (gene encoding for } \beta \text {-MHC protein) } \\ \text { MYL2 } & \text { Myosin Light Chain 2 (gene encoding for MLC2v protein) } \\ \text { NKX2-5 } & \text { NK2 Transcription Factor Related, Locus 5 } \\ \text { NANOG } & \text { NPPA }\end{array}$




$\begin{array}{ll}\text { NE } & \text { Norepinepherin } \\ \text { NET } & \text { Norepinephrin Plasma Membrane Transporter } \\ \text { NEUROD1 } & \text { Neuronal Differentiation 1 } \\ \text { OFT } & \text { Out Flow Tract } \\ \text { OCT4 } & \text { Octamer-binding Transcription Factor 4 (POU Class 5 Homeobox 1) } \\ \text { OASIS } & \text { CREB3L2 cAMP Responsive Element Binding Protein 3-like 1 } \\ \text { PRx2 } & \text { Paired Related Homeobox 2 } \\ \text { P/S } & \text { Penicillin/Streptomycin } \\ \text { PBS } & \text { Phosphate Buffered Saline } \\ \text { PDMS } & \text { Polydimethylsiloxane } \\ \text { PCR } & \text { Polymerase Chain Reaction } \\ \text { PP } & \text { Pacemaker Potential } \\ \text { PVDF } & \text { Polyvinylidene Flouride } \\ \text { PLN } & \text { Phospholamban } \\ \text { Pax6 } & \text { Paired Box 6 } \\ \text { qPCR } & \text { Quantitative PCR } \\ \text { R-SMAD } & \text { Receptor-regulated SMAD } \\ \text { RGM } & \text { Repulsive Guidance Molecule } \\ \text { RPMI } & \text { Rosewell Park Memorial Institute Medium } \\ \text { RPM } & \text { Resting Membrane Potential } \\ \text { RNA } & \text { Ribonucleic Acid } \\ \text { RT } & \text { Room Temperature } \\ \text { RYR2 } & \text { Ryanodine Receptor 2 } \\ \text { SV } & \text { Sinus Venous } \\ \text { SOX17 } & \text { SRY (Sex Determining Region Y)-Box 17 } \\ \text { SHF } & \text { Second Heart Field } \\ \text { SBE } & \text { SMAD- Binding Element } \\ \text { Snai1 } & \text { Snail Family Zinc Finger 1 } \\ \text { Smurf1 } & \text { SMAD Specific E3 Ubiquitin Protein Ligase 1 } \\ \text { SMAD } & \text { SCN5A }\end{array}$




$\begin{array}{ll}\text { SERCA } & \text { Sarco/Endoplasmic Reticulum Calcium-ATPase } \\ \text { SOTA } & \text { Self-organizing Tree Algorithm } \\ \text { SDS-PAGE } & \text { Sodium Dodecyl Sulfate-Polyacrylamide Gel Electrophoresis } \\ \text { SEM } & \text { Standord Error of Mean } \\ \text { SSC } & \text { Side Scatter Intensity } \\ \text { TBX } & \text { T-box Transcription Factor } \\ \text { TGF } \beta-1 & \text { Transforming Growth Factor } \beta \text {-1 } \\ \text { TIEG } & \text { Also Known as Kruppel-like factor 10 (KLF10) } \\ \text { TNNT2 } & \text { Troponin T Type 2 } \\ \text { TNNI3 } & \text { Troponin I3, Cardiac Type } \\ \text { Trb3 } & \text { Tribbles Homologue 3 } \\ \text { TBST } & \text { Tris-Buffered Saline and Tween 20 } \\ \text { TH } & \text { Tyrosine Hydroxylase } \\ \text { Tyr } & \text { Tyrosine } \\ \text { VMAT } & \text { Vesicular Monoamine Transporter } \\ \text { VEGF } & \text { Vascular Endothilial Growth Factor } \\ \text { VE-Cadherin } & \text { Vascular Endothelin - Cadherin } \\ \text { WNT } & \text { Wingless } \\ \text { WT1 } & \text { Wilms Tumor 1 }\end{array}$




\section{Introduction}

Congenital heart defects (CHDs) are the leading cause of birth-related deaths affecting 1214/1000 births in the Western industrialized world (Hoffman and Kaplan, 2002). The anatomical malformations may affect the walls, chambers or adjacent blood vessels. The etiology of most CHDs is unknown with approximately $20 \%$ being attributed to genetic mutations. Modeling of human embryonic cardiogenesis in vitro may help to go in mechanistic insight in early cardiac developmental events including events causing cardiac malformations.

The discovery of human embryonic stem (hES) cells (Thomson et al., 1998) and human induced pluripotent stem (hIPS) cells (Takahashi et al., 2007; Yu et al., 2007) has revolutionized our toolkit to study embryogenesis and subsequent organogenesis and significant effort has been invested in improving methods for efficient and reproducible stem cell differentiation towards a cardiac lineage.

\subsection{Embryonic Heart Development}

\subsubsection{Morphological changes during heart development}

Five days post fertilization, the blastocyst is formed, containing the inner cell mass from which the embryo arises, and the trophoblast (an outer layer of cells that later forms the syncytiotrophoblast to support the implantation of the embryo into the uterine wall). The proliferation of cells within the inner cell mass gives rise to the hypoblast and the epiblast; the former forms the yolk sac which does not contribute to any part of the newborn while the latter splits to separate the embryonic epiblast from the amniotic ectoderm. The formed amniotic cavity is then filled with amniotic fluid which is thought to operate as a "shock absorber" for the developing embryo (Gilbert, 2000). The embryonic plate initially known as the bilaminar germ disc is oval-shaped and situated between the yolk sac and the amnionic cavity (Fig. 1A). In the middle of this disc, the primitive streak that originated from the anterior epiblast is found with a rounded node (Hensen's node) at its cranial end (Moorman et al., 2003). Formation of this primitive streak is the first hallmark feature of gastrulation. 


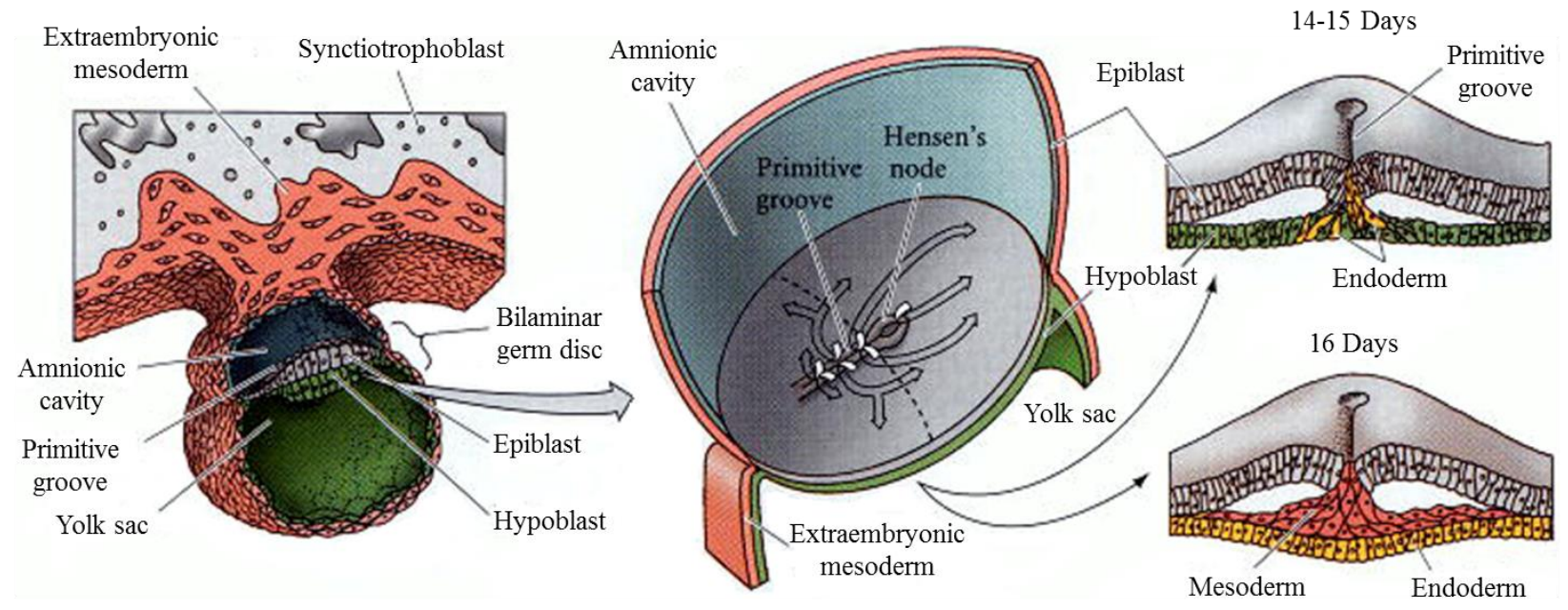

Figure 1: The gastrulation process.

(A) The embryonic plate initially known as the bilaminar germ disc is oval-shaped and situated between the yolk sac and the amnionic cavity. In the middle of this disc, the primitive streak that originated from the anterior epiblast is found with a rounded node (Hensen's node) at its cranial end. Gastrulation begins at the posterior end of the embryo, when epiblast cells migrate through the primitive streak. (B) Formation of the primary germ layers after gastrulation. Image taken from Gilbert (2000).

In humans, this process commences around the third week post-fertilization when the embryonic epiblast cells migrate through the streak and form the three germ layers: the ectoderm, the mesoderm and the endoderm. The first wave of cells migrating through the streak displaces the hypoblast cells and forms the endoderm which gives rise to the future gut derivatives (such as gastro-intestinal tract, lung and liver). The second wave of cells populates the mesodermal layer which is situated between the endoderm and upper layer of the epiblast (Fig. 1B). The mesoderm spreads laterally and cranially within the newly formed tri-laminar embryonic disc ultimately giving rise to three subpopulations: (1) the paraxial mesoderm as precursors for the somites, bone and cartilage, (2) intermediate mesoderm as precursors for the reproductive system and kidney and (3) the lateral plate mesoderm as precursors for blood, smooth muscle, heart and spleen. The remaining epiblast cells form the ectoderm which will form the surface ectoderm (epidermis) and the neural ectoderm (such as sensory organs, neural tube and neural crest).

With the formation of the lateral plate mesoderm, the cells migrate cranially and laterally to form two bilateral heart fields which achieve a crescentic shape at the midline of the embryonic 
disc. As the ectoderm transforms to a folded neural tube which will give rise to the developing brain coupled with invagination of the endoderm to form the foregut, the heart field precursors move ventrally and also fold into the primary endocardial tube of the heart enveloped by a myocardial epithelium (Harvey, 2002; Moorman et al., 2003). Note that the folding inverts the orientation of the developing heart, taking the shape of an inverted "Y" with the inflow region located caudally and the outflow region positioned cranially (Fig. 2). Next, the linear tube elongates as second heart field cells derived from the cardiac crescent populate both poles of the tube forming cranially the outflow tract (Takasato et al., 2014) and the primordium of the right ventricle and caudally the sinus venosus (Uhlen et al., 2015), which later becomes associated with the newly incorporated primary atrium (Kelly et al., 2001; Mjaatvedt et al., 2001; Waldo et al., 2001). The extensive elongation of the tube causes the developing heart to adopt a spiral shape and sweep rightwards in a process termed cardiac looping. This, in turn, forces the atria to migrate cranially and ultimately become positioned above the ventricles.

By human embryonic day 32, the heart tube is composed of atrial and ventricular components along with an outflow tract after which complex remodeling and heart division by septation and valve development forms the four-chambered heart; briefly, endocardial cushions arise which are precursors of the various valves of the adult heart (bicuspid and tricuspid valves in the atrioventricular canal as well as the aortic and pulmonary valves). These cushions also divide the outflow tract into the aorta and pulmonary artery via the aorticopulmonary septum (Harvey, 2002). Other features of remodeling involve the projection of a spongi-like layer of myocytes also known as trabeculae carneae from the inner surface of the ventricles along with the fusion of the inter-atria and inter-ventricular septae with the atrioventricular septum therefore forming the definitive cardiac chambers. This complex nature of cardiac development (differentiation and morphogenesis) poses the need to address the fundamental molecular determinants underlying this process. 


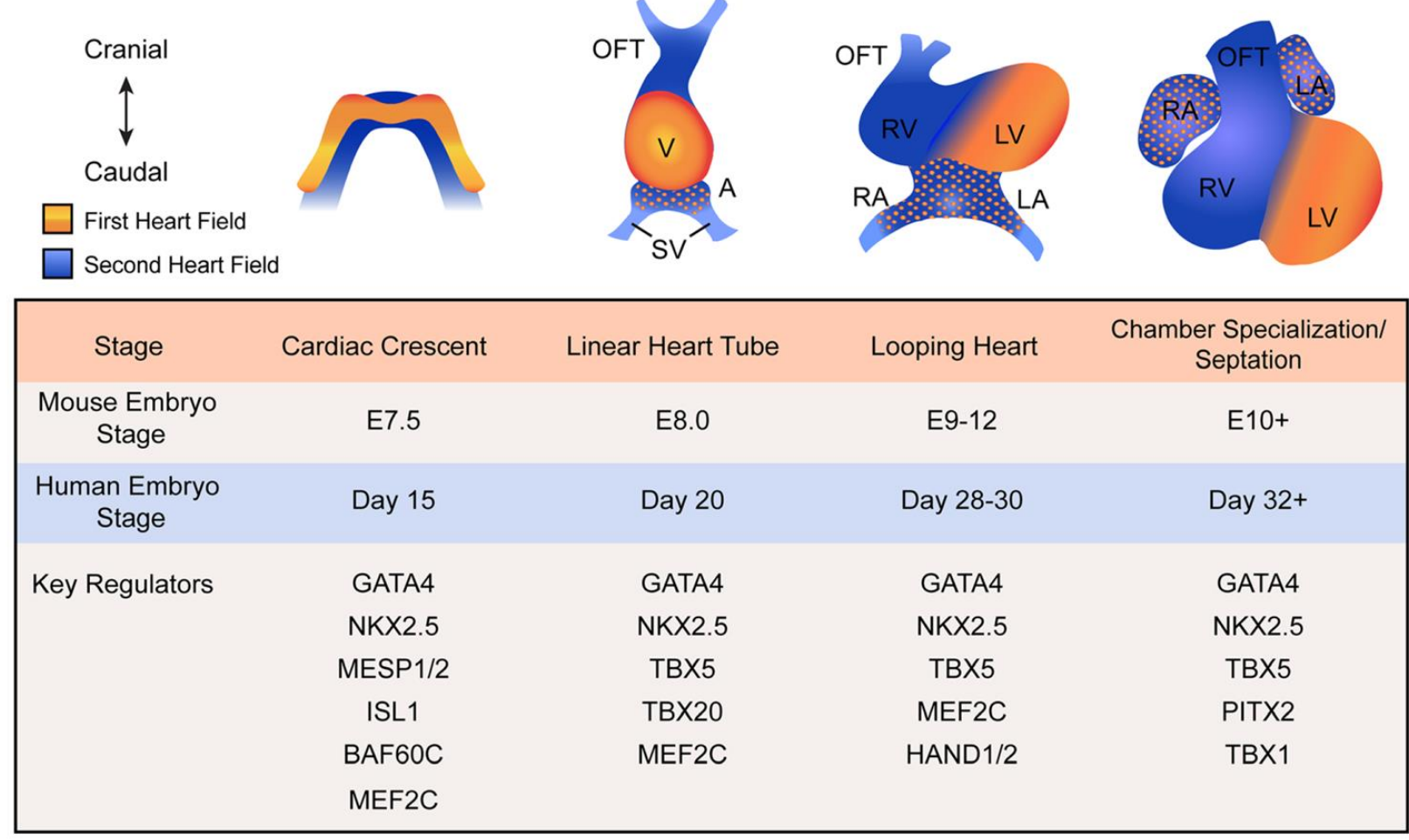

Figure 2: Overview of the formation of the four-chambered heart.

The cardiac crescent is formed from the first and second heart fields (FHF and SHF, respectively) at the end of gastrulation. Differentiation of FHF progenitors forms the linear heart tube, which gives rise to the left ventricle and a part of the atria. Meanwhile, SHF progenitors contribute to the formation of the right ventricle and the outflow tract. The extensive elongation of the tube causes cardiac looping which forces the atria to migrate cranially and ultimately become positioned above the ventricles. Complex remodeling and heart division by septation and valve development forms the four-chambered heart. Key gene regulators governing this complex process are listed. LA: left atria; LV: left ventricle; OFT: outflow tract; RA: right atrium; RA: right atrium; SV: sinus venosus. Image taken from Paige et al. (2015).

\subsubsection{Molecular changes during heart development}

As aforementioned, shortly after gastrulation the three primary germ layers form the endoderm, ectoderm and mesoderm (Fig. 1). Studies in mice have shown that cells from the primitive streak, marked by the T-box transcription factor $T$ (Brachyury), migrate through the anterior sections of the streak to generate cardiac mesoderm (Murry and Keller, 2008). These precursor cells express the basic helix-loop-helix transcription factor mesoderm posterior 1 (Mesp1) (Bondue et al., 2008; Burridge et al., 2012), which is in turn activated by the expression of the T-box transcription factor Eomesodermin (Costello et al., 2011; Paige et al., 2015; van den Ameele et al., 2012). Being the master switch for early cardiac specification, the transient expression of Mespl rapidly activates the expression of cardiac transcription factors such as 
NK2 homeobox $5(N k x 2-5)$, myocyte enhancer factor 2C (Mef2c), heart and neural crest derivatives expressed 2 (Hand2), and GATA binding protein 4 (Gata4). It also appears to be involved in the downregulation of genes implicated in either maintaining pluripotency such as octamer-binding transcription factor 4 (Oct4) and nanog homeobox (Nanog) or in other endodermal cell fate determination such as SRY (sex determining region Y)-box 17 (Sox17) and forkhead box A2 (Foxa2) (Bondue et al., 2008; Paige et al., 2015).

Mespl is only transiently expressed as an early cardiac fate regulator. Subsequently, cardiac progenitor cells continue to migrate and form the cardiac crescent with the expression of T-box transcription factor $(T b x) 5$ and hyperpolarization activated cyclic nucleotide gated potassium channel 4 (Hcn4), marking the first heart field (FHF) cells (Liang et al., 2013; Takeuchi et al., 2003). Conversely, Islet LIM homeodomain 1 (Isl1) labels the second heart field (SHF) cells (Cai et al., 2003; Kelly, 2012). The elongation of the linear heart tube via the addition of SHFprecursors to both poles appears to be primarily mediated by Tbxl expression (Rana et al., 2014), which in turn activates fibroblast growth factors (Fgf) 3, 8 and 10 (Park et al., 2006; Watanabe et al., 2012; Zaffran and Kelly, 2012).

Following the formation of the primitive heart tube, cardiac chamber morphogenesis and specification is induced. This is regulated by multiple transcription factors including $N k x 2-5$, Gata4 and in a narrower pattern the T-box family; briefly, Tbx2 and Tbx3 are expressed in the outflow tract and atrioventricular canal (Rana et al., 2013). Tbx5 has a peak expression in the inflow tract and primitive atrium (Bruneau et al., 1999). It has been shown to interact with Tbx20 (Hoogaars et al., 2007) and other cardiac transcription factors to promote induction of atrial natriuretic factor (Nppa) and gap junction proteins connexin 40 and 43 (Cx40 and Cx43 respectively). With further myocardial chamber specification, myosin light chain (Mlc) $2 \mathrm{a}$ and $2 \mathrm{v}$ are transcribed marking the atria and ventricles, respectively (Small and Krieg, 2004), along with other cardiac structural proteins such as $\alpha$-actinin, $\alpha$-myosin heavy chain $(\alpha-\mathrm{MHC})$, or cardiac Troponin-T (cTnT) (Mummery et al., 2012).

The information obtained from embryonic heart development, turned out to be instrumental for the development of protocols to direct differentiation of pluripotent stem cells to cardiomyocytes in the dish. 


\subsection{Directed Cardiac Differentiation of Stem Cells}

After the first report on the isolation and culture of hES cells in 1998 (Thomson et al., 1998), it was demonstrated that hES would spontaneously differentiate in suspension to embryoid bodies (EBs), containing in $\sim 8 \%$ of the EBs beating cardiomyocytes (Kehat et al., 2001). Currently, two approaches are widely utilized for the cardiac differentiation of hES cells (Fig. 3): (1) differentiation in EB comprised typically of defined cell numbers or (2) 2-dimensional (2D) cultures (Burridge et al., 2012). The low cardiomyocyte yield is a key limitation of the EB protocol and cultures containing 20\% fetal bovine serum (FBS) (Kehat et al., 2001). The differentiation efficiency improved up to $50 \%$ with the addition of growth factors such as bone morphogenetic protein (BMP)-4 (Takei et al., 2009) or wingless-type (Wnt)-3A (Tran et al., 2009) during the mesodermal induction period. 


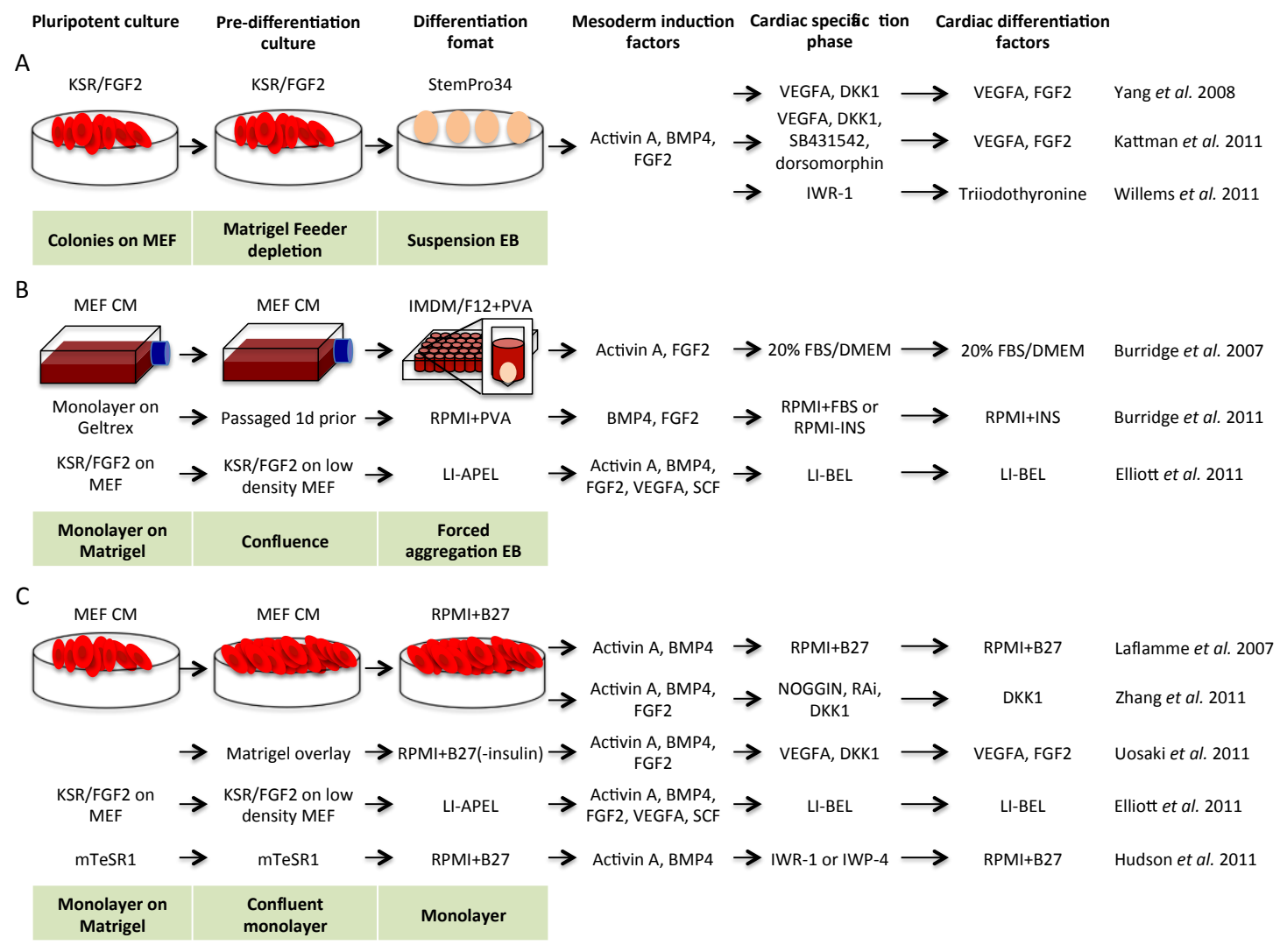

Figure 3: Current methods to differentiate of hPS cells.

Overview includes the cell culture and differentiation format with the addition of cardiac induction and specification factors. (A) Suspension of EBs; (B) forced aggregation of EBs; (C) monolayer differentiation. KSR: knock serum replacement; FGF2: fibroblast growth factor-2; BMP4: bone morphogenetic protein 4; VEGF A: vascular endothelial growth factor A; DKK1: dickkopf homolog 1; SB431542: TGF $\beta$-1/Activin/NODAL signaling inhibitor [ALK4,5,7]; dorsomorphin: BMP signaling inhibitor [ALK2,3,6]; IWR-1: inhibitor of Wnt response-1; MEF CM: mouse embryonic fibroblast conditioned hESC medium; RPMI1640: rosewell park memorial institute 1640 basal medium; B27: media supplement; NOGGIN: BMP signaling inhibitor; Rai: retinoic acid signaling inhibitor; LIAPEL: low insulin albucult polyvinyl alcohol essential lipids media; SCF: stem cell factor KITLG; LIBEL: low insulin bovine serum albumin essential lipids media; Y27632: rho kinase inhibitor; CHIR99021: GSK3 inhibitor; IWP-2: inhibitor of Wnt production-2; FBS: fetal bovine serum; DMEM: dulbecco's modified eagle's medium; RPMI+PVA: RPMI based media supplemented with polyvinyl alcohol; RPMI-INS: RPMI based media without insulin. Image adapted from Burridge et al. (2012).

Determining the effect and timing of various growth factors on cardiac differentiation was further studied in serum free culture using the EB-based approach. The addition of low concentrations of BMP4, FGF-2 and Activin-A for mesodermal induction followed by cardiac specification with vascular endothelial growth factor (VEGF)-A and dickkopf Wnt signaling pathway inhibitor 1 (DKK1) produced contracting cardiomyocyte population whilst maintained under hypoxic conditions (Yang et al., 2008). The subsequent inhibition of TGF $\beta /$ Activin/Nodal 
and BMP signaling using small molecules (between day 3-day 5) or Wnt-inhibition (between day 4-day 10) enhanced the protocol (Fig. 3A) (Kattman et al., 2011; Willems et al., 2011). Another protocol introducing the forced-aggregation method followed by mesoderm induction using BMP4 and FGF-2 also increased the efficiency of cardiac differentiation to more than 90\% (Burridge et al., 2011). In a recent attempt to generate a homogeneous population of ventricular cardiomyocytes without genetic manipulation or cell sorting, chemical modulation of Wnt signaling using inhibitor of Wnt response (IWR)-1 increased expression of cardiac specific marker troponin T type 2 (TNNT2) to 89\% coupled with a 56-fold increase in MLC2v expression (Karakikes et al., 2014) in comparison to the DKK1-treated cells as described by Yang et al. (2008).

Culturing cells in monolayer has also been employed to reduce the complex microenvironment of EBs (Fig. 3C). The relatively uniform layer of cells permits better control of spatial cell arrangement (Hudson et al., 2012), and decreases diffusion barriers. Thus, the addition of growth factors and other interventions will have greater control and reproducibility (Mummery et al., 2012). This was effective in H7-hES cells that generated $>70 \%$ cardiomyocytes relative to $30 \%$ with the EB-based approach when exposed to Activin-A and BMP4 in serum-free conditions (Paige et al., 2010). The inhibition of Wnt signaling enhanced cardiac specification to primitive streak cells in monolayer (2D) culture compared to EB differentiation (Hudson et al., 2012). Moreover, in an attempt to decrease line to line variability, the same group has shown that single-cell culture of hES in a defined medium (mTeSR-1) allowed higher efficiency of cardiac differentiation (Hudson et al., 2012).

Despite improved differentiation efficiency, it was the use of defined differentiation protocols following known in vivo developmental pathways which has led to robust and efficient cardiac differentiation protocols free from contaminating cell types (Murry and Keller, 2008). This is highlighted by the importance of Activin-A to switch cell fate to a mesodermal lineage instead of to a definitive endodermal lineage when introduced at low concentrations. Concurrently, BMP4 enhanced mesodermal specification in CHIR99021-induced cardiomyogenesis (Kim et al., 2015). 
Currently known differentiation methodologies all report spontaneously beating areas composed of stem cell-derived cardiomyocytes with an immature phenotype on the morphological, molecular and functional levels (Rajala et al., 2011). The structural architecture reveals cells to be rarely elongated to a rod-shaped morphology, being the typical phenotype of adult cardiomyocytes (Snir et al., 2003). Despite showing sarcomeres with A-, I- and Z-bands and intercalated discs with gap junctions analogous to the adult cardiac cells, the sarcomeric organization remained immature (Snir et al., 2003) with a highly heterogeneous degree of myofibril bundle organization (Kehat et al., 2001). On the molecular level, the stem cell-derived cardiomyocytes reveal expression of transcription factors, including Nkx2.5, GATA-4, Isl-1 as well as structural proteins, including cardiac troponins T and I, $\alpha$-actinin, MHC, MLC2a and MLC2 $\mathrm{v}$ which suggests the presence of a mixture of ventricular and atrial-like cardiac cells with diverse ratios depending on the differentiation protocol utilized (Hudson et al., 2012; Kehat et al., 2001; Norstrom et al., 2006; Yoon et al., 2006).

Stem cell-differentiated cardiomyocytes reveal immature action potentials (AP) characterized by lower upstroke velocity and automaticity (He et al., 2003; Rajala et al., 2011). The absence of an inward rectifier potassium current, as detected with global expression profiling, may explain their spontaneous contractility (Xu et al., 2009). Voltage clamp studies also support these observations (Brito-Martins et al., 2008). Irregular intracellular calcium handling may be explained by the lack of phospholamban and calsequestrin proteins in the sarcoplasmic reticulum (Binah et al., 2007; Dolnikov et al., 2005; Sartiani et al., 2007). Additionally, hESderived cardiomyocytes display a negative force-frequency relationship as opposed to native adult myocytes (Kehat et al., 2002).

Additional research is needed to develop strategies for enhancing the maturity of stem cellderived cardiomyocytes in vitro. Several groups suggested the use of tissue engineering to better simulate heart muscle development and maturation over monolayer cultures (Zimmermann, 2011). 


\subsection{Tissue Engineering: Basis for Modeling Heart Development in a Dish}

Tissue engineering has many potential applications, including organ repair, disease modeling and drug development. The first macroscopic engineered myocardium was constructed using embryonic chick cardiomyocytes mixed with a collagen hydrogel and utilized as a test bed for genetic manipulation (Eschenhagen et al., 1997). Matrigel ${ }^{\mathrm{TM}}$ for the provision of additional extracellular matrix components and the application of mechanical strain were identified as essential factors for the translation of the avian to a mammalian model of engineered heart muscle (Zimmermann et al., 2000). Other tissue engineering approaches include cardiac cell seeding on preformed scaffolds (Carrier et al., 1999) and stacking of myocyte sheets to form a multi-layered muscle (Shimizu et al., 2002) (Fig. 4).

Ideally, these engineered tissues should mimic the native myocardial environment. A key feature of advanced myocardial tissue engineering formats is their anisotropic contractile performance. This is typically coupled with advanced sarcomere organization and longitudinal cardiomyocyte orientation in comparison to $\mathrm{EB}$ and $2 \mathrm{D}$ cultures. Enhanced maturation of neonatal rat cardiomyocyte-based engineered heart muscle (EHM) was evidenced by a shift from skeletal to the cardiac actin isoform alongside MLC2v expression (Tiburcy et al., 2011). The presence of non-myocytes in EHM was found to be important for advanced functional maturation (Naito et al., 2006). This was confirmed in stem cell based engineered myocardium (Didié et al., 2013; Jackman et al., 2015; Kensah et al., 2012; Zhang et al., 2013). In addition to mechanical stimulation, it appeared that electrical stimulation can advance tissue maturation (Godier-Furnemont et al., 2015; Nunes et al., 2013; Radisic et al., 2004). 
Cell source

- mESC derived CM

- hESC derived CM

Modified cell composition

- $\quad$ +/- Preplating

- Endothelial cells

- Fibroblast/MSC

Mode of stretching

- Static

- Phasic

- Auxotonic

\section{Stretching device}

- Elastic silicone post on silicone frame

- Velcro frame

- Flexcell

- Laminin-coated braided silk suture

- Silicone stamps on bottom of dish

- Motorised phasic stretch of rings

- Rings hooked onto spring-mounted device

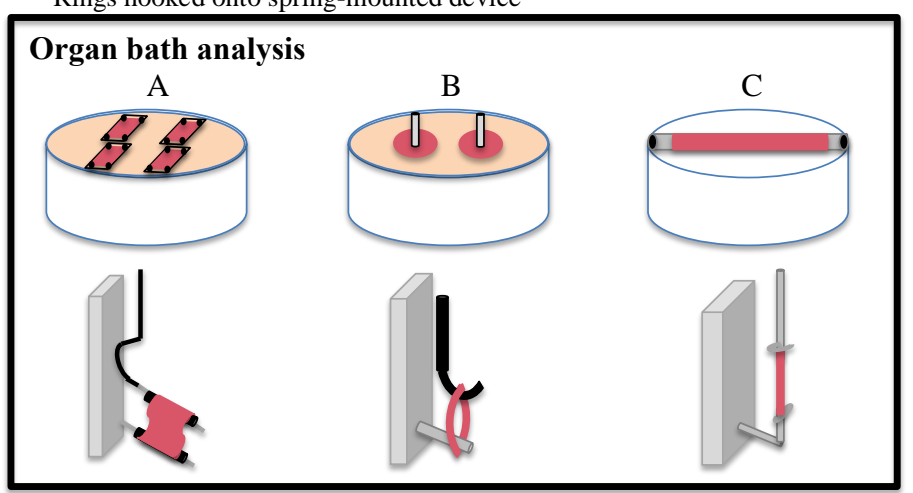

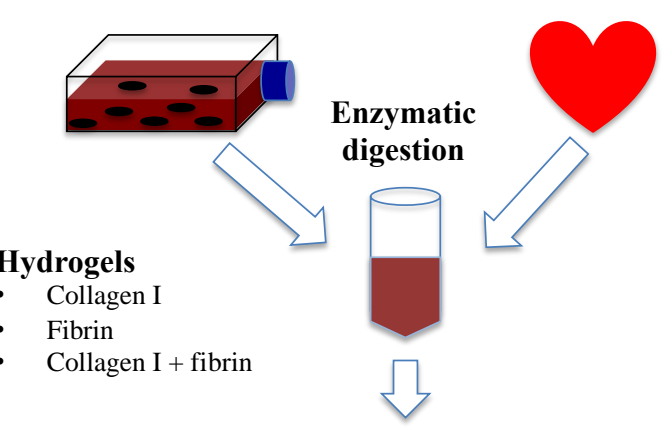

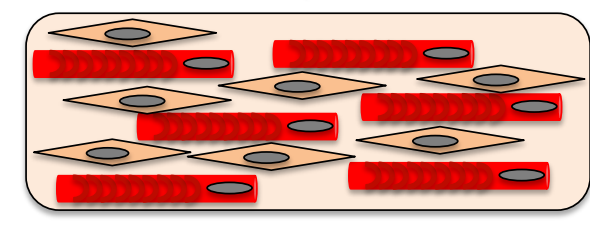

Quantification of contractile force
Optical analysis

D

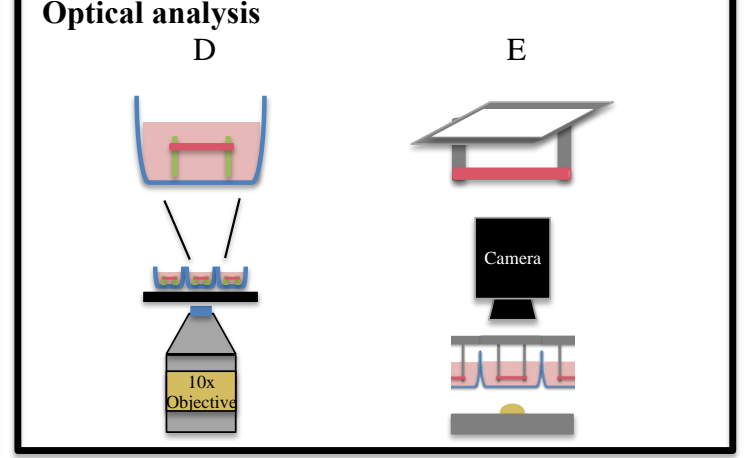

Cell source

- NRCM

- NMCM

- Embryonic chicken CM

Casting mold

- Ring format silicone/teflon mold

- Ring format teflon mold

- Strip format agarose

- Rectangle silicone

- $\quad$ Silicone mold with mesoscopic-sized posts

Silicone and laminin-coated 6 well cell culture dish

\section{Modifiers}

- Matrigel

- Ambient oxygen concentration

- Oxygen carrier

- $\quad$ Defined medium

Figure 4: Overview of tissue engineering approaches, cell sources and contractility evaluation.

Cells from various sources are mixed within hydrogels and cast into molds of several shapes and sizes. Tissue construct quality can be further enhanced using mechanical and electrical stimulation. Contractile force is either measured in (A, B and $\mathbf{C}$ ) an organ bath or using (D and E) optical recordings. NRCM: neonatal rat cardiomyocyte; NMCM: neonatal mouse cardiomyocyte; hESC: human embryonic stem cells; CM: cardiomyocytes; MSC: mesenchymal stem cells. Image adapted from Eschenhagen et al. (2012).

With the exciting progress of stem cell research and advances in tissue engineering approaches, active research has recently embraced the concept of the generation of stem-cell based organoids directly from undifferentiated pluripotent stem cells supported to self-assemble in a three-dimensional culture environment (Yin et al., 2016). A wide variety of organoids has been recently developed including liver (Huch et al., 2013; Takebe et al., 2013), kidney (Takasato et al., 2014), prostate (Karthaus et al., 2014) and brain (Lancaster et al., 2013). The latter, for instance, was generated as droplets of matrigel containing neuroectodermal tissues which further differentiated into distinct brain regions such as the dorsal cortex, ventral telencephalon and choroid plexus (Lancaster et al., 2013). Yet so far no data have been reported for the 
development of a macro-tissue with functional properties of bona fide myocardium (i.e.: cardiac organoid). The following section introduce the concept of a bioengineered heart muscle (BHM) developed by our lab as a model to simulate human heart development in a dish. 


\subsection{Bioengineered Heart Muscle: Concept and Preliminary Data}

Inspired by embryonic development and advances in tissue engineering, we postulated whether hPSCs can be dirrected to differentiate and self-assemble into a BHM. This process would eventually require the differentiation of hPSCs not only into cardiomyocytes, but also supportive stromal cell populations. Proof-of-concept for directed self-assembly of hPSC into BHM was developed prior to the start of the thesis (Hudson et al., in revision). Here it was demonstrated that hPSC after mixing with a collagen hydrogel could be subjected to a defined serum-free stage specific stimulation protocol to form spontaneously contracting BHMs by day 13 of culture. The stimuli (growth factors and small molecules) were chosen to manipulate key signaling pathways important to recapitulate native heart development. By day 22 of culture, these tissues developed networks of elongated and striated cardiomyocytes (Fig. 5A) and positive inotropic response to increasing extracellular calcium concentrations (Hudson et al., in revision; Fig. 5B).

BHM function was further enhanced with the addition of (1) ascorbic acid 2-phosphate (Asc2P), (2) TGF $\beta-1$, (3) adjusting extracellular calcium concentrations from $0.4 \mathrm{mmol} / \mathrm{L}$ to physiological levels and (4) dynamic mechanical stimulation (Hudson et al., in revision). This early data provides proof-of-concept that differentiation of stem cells into cardiomyocytes first is not necessary for cardiac tissue engineering and can be avoided if systemic control over the assembly and differentiation of hPSCs into cardiac organoids is possible. Yet, this model needed further characterization and optimization before it can be introduced as a robust tool for the simulation of heart development and disease phenotypes. 
A

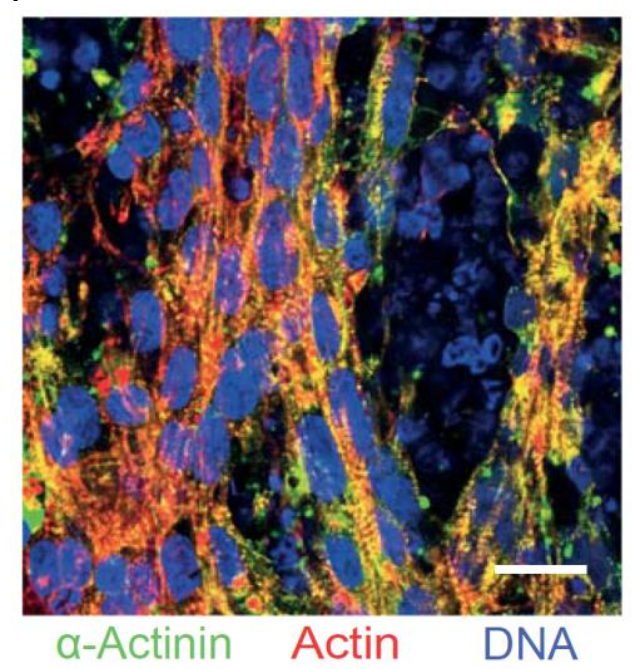

B

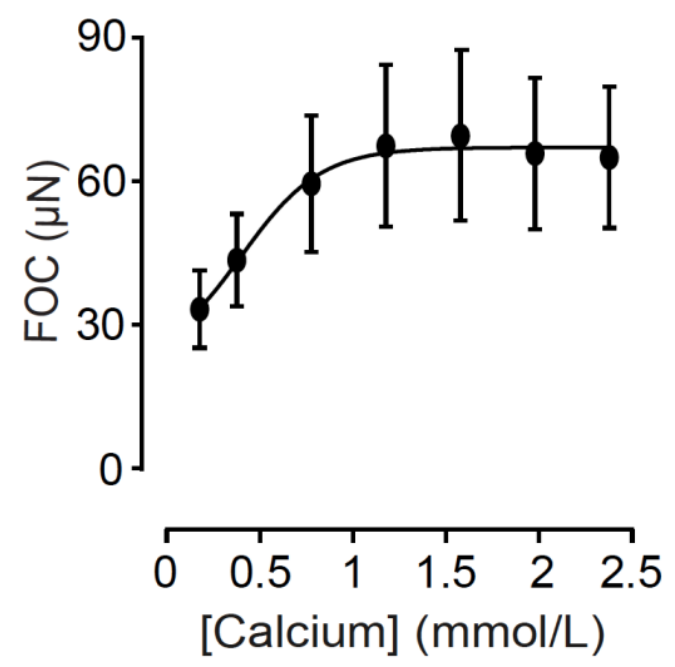

Figure 5: Morphological and functional assessment of BHM at day 22.

(A) Whole-mount staining for $\alpha$-actinin (green), actin (red) and DNA (blue) showing muscle bundle formation; scale bar: $20 \mu \mathrm{m}$. (B) Isometric force measurement of BHM showing a positive inotropic response to increasing extracellular calcium under $3 \mathrm{~Hz}$ electric stimulation $(n=7)$. FOC: force of contraction. Images taken from Hudson et al. (in revision).

We postulated that modulation of key signaling pathways during early heart development may influence BHM development. We sought to target four major pathways that are implicated in the early cardiac induction of stem cells including TGF $\beta /$ Activin/Nodal, BMP, WNT and FGF. From those factors, BMPs appear to be particularly crucial for the induction of cardiac mesoderm (Burridge et al., 2011; Hudson et al., 2012; Laflamme et al., 2007; Yang et al., 2008; Yao et al., 2006). 


\subsection{Bone Morphogenetic Protein Signal Transduction}

\subsubsection{BMP signaling cascade: ligand and pathway}

BMPs were first identified in the formation of bone and cartilage (Wozney et al., 1988). Ligands of this family can be further divided based on similarity in amino acid sequence, target genes and biological activity. However each ligand targets different genes, BMP2, for example, promotes extraembryonic endoderm differentiation, whilst BMP4 evokes differentiation into mesoderm in hES cultures (Okita and Yamanaka, 2006). In the absence of BMP, the type-II and type-I transmembrane serine/threonine kinase BMP receptors (BMPR-II and BMPR-I respectively) appear as preexisting homo and heterodimers on the cell membrane (Fig. 6)

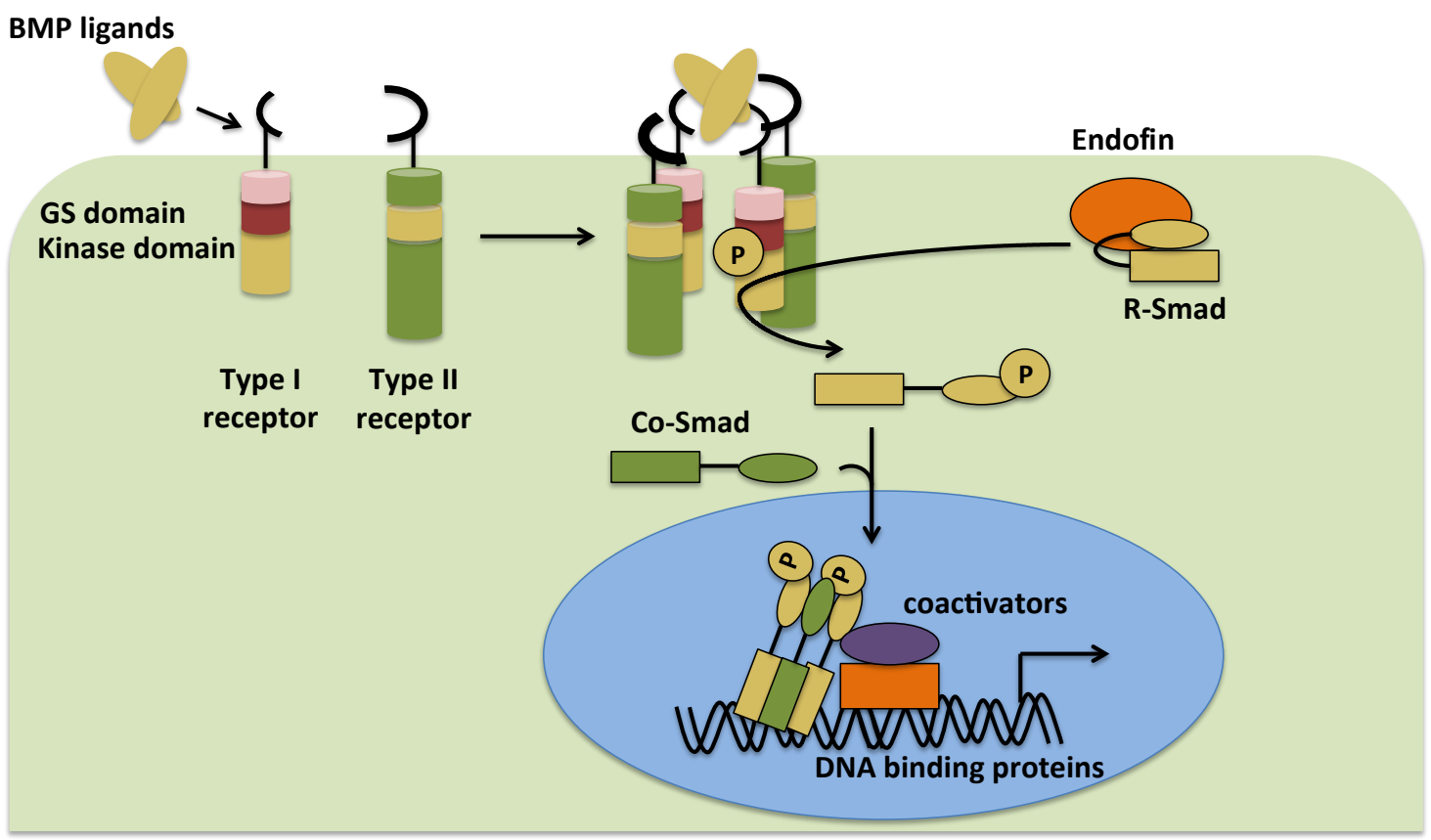

Figure 6: BMP signaling pathway.

BMP ligands bind to the type-II receptors and induce complex formation with type-I receptors which are consequently phosphorylated at their GS domain by the active kinase domain of the type II receptor. R-SMADs interact with membrane anchoring proteins (e.g. endofin). The activated BMP type-I receptors then phosphorylate R-SMADs, which then formed a complex with Co-SMAD, translocate to the nucleus and regulate gene transcription via interacting with DNA binding proteins. BMP: bone morphogenetic protein; GS domain: glycineserine amino acid rich domain; SMAD: small body size mothers against decapentaplegic; R-SMAD: receptorregulated SMAD; Co-SMAD: common-mediator SMAD. Image adapted from Miyazono et al. (2010). 
Upon ligand binding, BMPR-I recruits BMPR-II inducing receptor oligomerization and complex formation. Consequently, the active type-II receptor phosphorylates the glycine-serine (GS) amino acid rich domain of BMPR-I at serine and threonine residues (Souchelnytskyi et al., 2002). Endofin, an endosomal protein, binds to activated type-I receptor at its FYVE anchoring domain thus recruiting inactive receptor-regulated small body size mothers against decapentaplegic (R-SMADs) at its mad homology (MH) 2 domain to the kinase domain of BMPR-I for subsequent phosphorylation at two serine residues (Okita and Yamanaka, 2006). Post-activation, R-SMADs undergo conformational changes that disrupt their interaction with endofin and cause their dissociation from receptor kinases (Qin et al., 2001) to bind with SMAD-4 in a 2:1 ratio at its C-terminus (Kawabata et al., 1998). The SMAD complex then translocates to the nucleus, recognizes GC rich sequences (Karaulanov et al., 2004; Truksa et al., 2009) via SMAD binding element (Uhlen et al., 2015) on SMAD-4 (Miyazono et al., 2010) and interacts with DNA binding proteins at its MH1 domain. DNA binding proteins such as p300 and CREB-binding protein (CBP) help in loosening nucleosomal structure and increase accessibility to transcriptional factors (Dijke et al., 2006) that are thus recruited by the SMAD complex at the $\mathrm{MH} 2$ domain.

The transcriptional machinery formed is essential for the regulation of BMP target genes. Several studies have revealed that BMP signaling induces the transcription of $O A S I S, P R x 2$, TIEG, Snail and more importantly inhibitor of differentiation (Id)1-3 proteins during mesenchymal osteoblastic differentiation (Hayashi et al., 2008). In most cell types, Id proteins are thought to be the primary target of BMP signaling. The aforementioned proteins inhibit basic-helix-loop-helix (bHLH) transcription factors that are known to upregulate cell differentiation by binding at their HLH dimerization motif (Miyazono and Miyazawa, 2002). Other targets of BMP signaling include numerous extracellular and intracellular proteins that enhance or inhibit the BMP pathway for example, noggin (Groppe et al., 2002; Sebald et al., 2004; Yuasa et al., 2005), dorsomorphin (Hao et al., 2008), and BMP-activin membrane-bound inhibitor (BAMBI) (Miyazono et al., 2010). Additionally, intracellular proteins like inhibitorySMADs (I-SMADs) and SMAD ubiquitin regulatory factor 1 (Smurf1) hinder signal transduction via degradation or binding. Conversely, receptor guidance molecules (RGM) (Babitt et al., 2005; Corradini et al., 2009; Miyazono et al., 2010; Xia et al., 2007) dragon 
(Samad et al., 2005) and tribbles-like protein 3 (Trb3) (Chan et al., 2007) function to enhance BMP signaling via ligand binding to membrane receptors (i.e.: RGM and dragon) or degradation of Smurfs intracellularly (i.e.: Trb3 protein).

The regulation of BMP signaling is complex and depends on many extra- and intracellular molecules that can inhibit/enhance the overall mechanism. Its function can additionally be affected by alternate signaling pathways such as TGF $\beta /$ Activin/Nodal, WNT and FGF. These parallel pathways partake in various cardiac developmental decisions.

\subsubsection{Role of BMP signaling during cardiogenesis}

In 1965, Urist et al. identified BMPs first as molecules that help in the induction of endochondral osteogenesis in vivo (Urist, 1965). However, knock-out mouse models revealed that the role of individual BMPs in development is not restricted to bone and cartilage, but central for embryogenesis as a whole and the formation of many organ systems such as skeletal, urinary, neurological and most importantly cardiovascular system.

BMPs play a crucial role during embryonic gastrulation and specifically also for cardiac development. Accordingly, a knock-out of BMP2/4 is embryonically lethal and BMP1/7/11 knockout mice die shortly after birth (Wang et al., 2014). Deficiency of BMP2 alone led to cardiac malformations particularly in the development of heart valves and septa (RiveraFeliciano and Tabin, 2006). This similar phenotype was also observed in BMP6 and 7 double knock outs (Kim et al., 2001). Other knockout studies revealed a role for BMP10 in cardiomyocyte proliferation (Chen et al., 2004). Moreover, the lack of both extracellular (i.e.: receptors) and intracellular (i.e.: $\mathrm{R} / \mathrm{Co} / \mathrm{I}-\mathrm{SMADs}$ ) regulators in mice results in embryonic lethality (Wang et al., 2014). SMAD1 mutants have defects in extraembryonic structures and formation of germ cells (Mishina et al., 1995; Tremblay et al., 2001). SMAD5 knockouts exhibit defects in left-right asymmetry (Chang et al., 2000).

BMP4 and its type-I receptor deficient mice lack the ability of mesodermal differentiation (Lawson et al., 1999; Mishina et al., 1995). Later it was shown that during the formation of the 
three germ layers, BMP4 is released from the extra-embryonic ectoderm to the anterior medial mesoderm with cardiogenic properties (Arnold and Robertson, 2009; Burridge et al., 2012; Tam and Loebel, 2007). This process occurs in a narrow time window that permits cells to transiently express the mesodermal marker Brachyury T (Zhu et al., 2013). Further long term exposure to BMP4 can result in trophoblast and extra embryonic endoderm differentiation (Zhang et al., 2008).

\section{In-vivo Heart development}

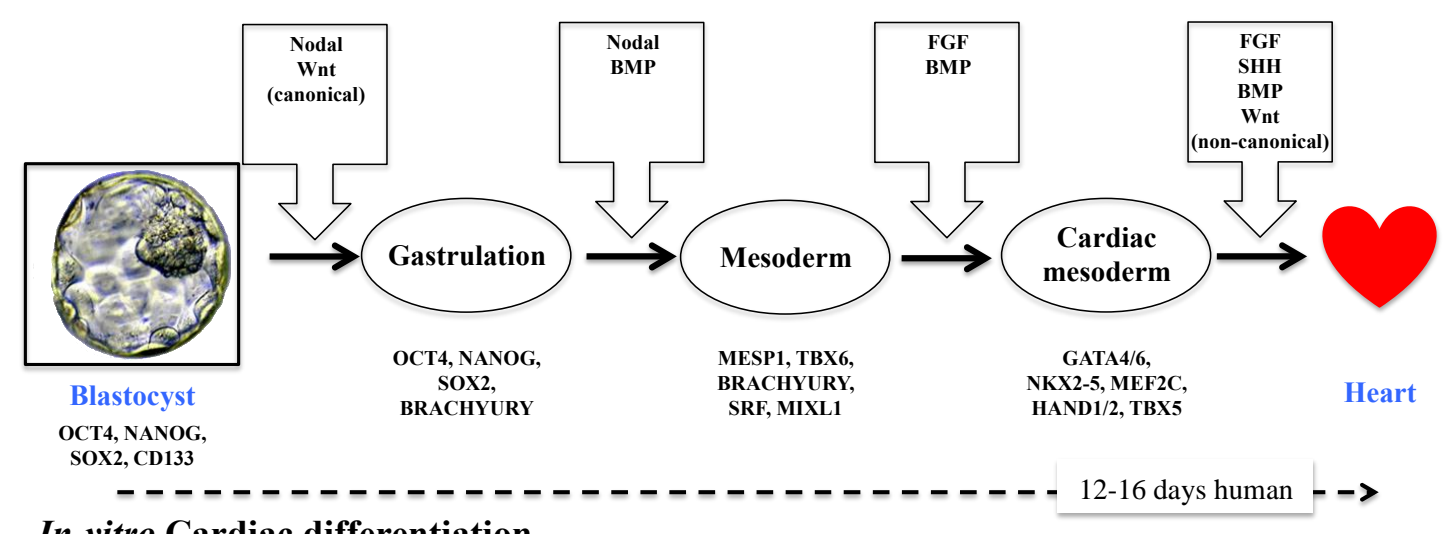

In-vitro Cardiac differentiation

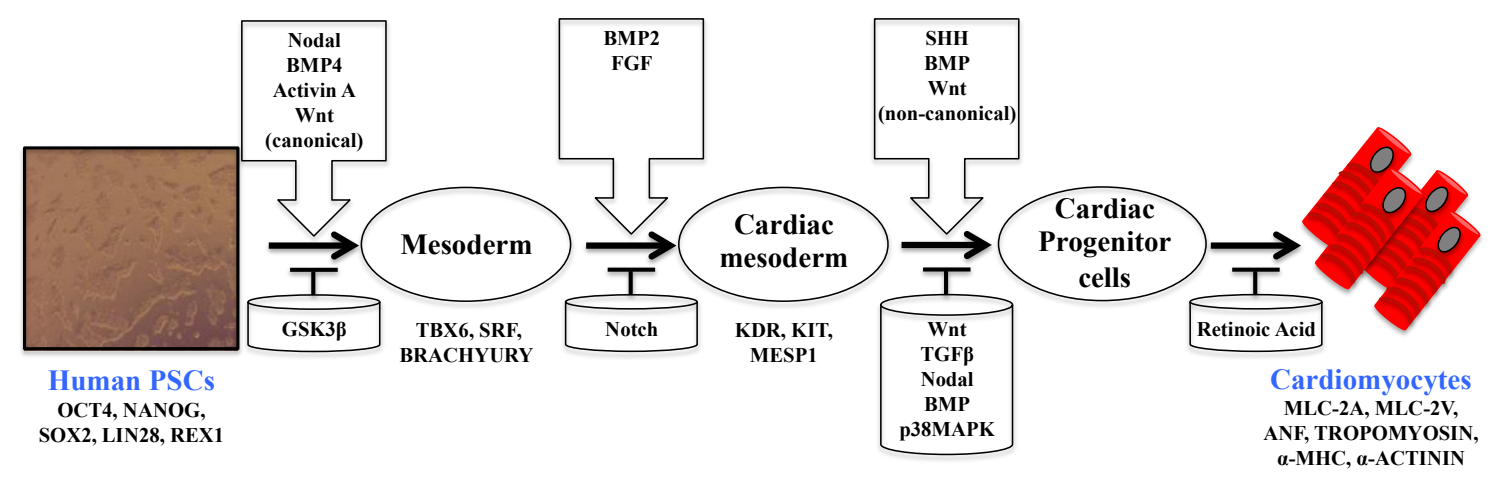

Figure 7: Signaling pathways and gene regulators governing cardiomyogenesis.

Wnt: Wingless signaling; BMP: bone morphogenetic protein signaling; FGF: fibroblast growth factor signaling; SHH: sonic hedgehog signaling; GSK3 $\beta$ : glycogen synthase kinase 3; TGF $\beta-1$ : transforming growth factor $\beta-1$; p38MAPK: p38 mitogen-activated protein kinases; PSCs: pluripotent stem cells. Image adapted from (Verma et al., 2013).

Stimulation of pre-cardiac mesoderm derived cell populations from hPSCs with BMP4 along with Wnt signaling inhibition induces cardiomyocyte specification. However, BMP4 activation 
alone causes the formation of epicardial cells expressing WT1 and TBX18 (Witty et al., 2014). Another role of BMP4 in cardiac lineage specification was demonstrated by Cagavi et al. (2014) where cardiac differentiation was enhanced upon the stimulation of cardiac progenitor cells expressing ISL1 with BMP4 via the activation of TBX5 and TBX20 normally expressed in the first and second heart fields (Cagavi et al., 2014). Additionally, and using mice knockouts it has been shown that following cardiac crescent formation and heart patterning, BMP signaling has a role in atrioventricular (Jiao et al., 2003) and outflow tract (Liu et al., 2004) septation. In zebra fish, BMP4 has a crucial role in directing cardiac asymmetry (Lenhart et al., 2013).

The recapitulation of cardiac differentiation in vitro at stage-specific windows has been made possible due to the extensive studies of the BMP pathway in animal models (Fig. 7). While the advancing differentiation methodologies are becoming highly defined and serum-free as discussed in section 1.2, challenges involving line-to-line variability between cells still remain, which ultimately affect the response to endogenous factors thus reducing the reproducibility and efficiency of cardiac differentiation. Several groups have demonstrated that the vast inconsistency in differentiation among pluripotent stem cell lines is likely due to the differences in initial state of pluripotency (Burridge et al., 2011; Hudson et al., 2012; Mummery et al., 2012) as well as genetic and epigenetic differences between hES cell lines (Adewumi O, 2007; Allegrucci and Young, 2007; Skottman et al., 2005). In addition, the protein factors used as culture medium supplement for directed differentiation can exhibit considerable batch-to-batch differences in biological activity. This technical challenge may be overcome by replacement of biological factors with small molecules that target the typically growth factor activated key pathways of cardiac differentiation.

Advantages of well characterized small molecules over protein-based regulators include their easy membrane diffusion, instant activation/inhibition of selective targets, reversible mode of action (Willems et al., 2009) and low cost in comparison to recombinant proteins (Wang et al., 2011). Furthermore, their potency, selectivity and solubility can be adapted as needed by chemical modulation (Willems et al., 2011). The synthesis and characterization of these discrete molecules remain the hope for generating a universal differentiation protocol that will help surpass cell-line variability and increase hPSC-derived cardiac output. 


\subsubsection{Chalcones: potential candidate for BMP4 replacement}

In a recent screen for small molecule activators of BMP signaling using luciferase assay under the Id1 promoter, four compounds out of 5600 molecules tested from different libraries belonging to the family of flavonoids induced luciferase activity greater than $50 \%$ relative to BMP4 (Vrijens et al., 2013). Two of these compounds were chalcones (isoliquiritigenin and 4'hydroxychalcone, Fig. 8A) and the other two were flavones (apigenin and diosmetin). Only the former compounds activated BMP signaling via the phosphorylation of SMAD 1/5 as well as the expression of Id 1 target gene of BMP pathway in a concentration dependent manner (Fig. $8 B)$.

A<smiles>O=C(/C=C/c1ccc(O)cc1)c1ccc(O)cc1O</smiles>

Isoliquiritigenin<smiles>O=C(/C=C/c1ccccc1)c1ccc(O)cc1</smiles>

4'-hydroxychalcone

B
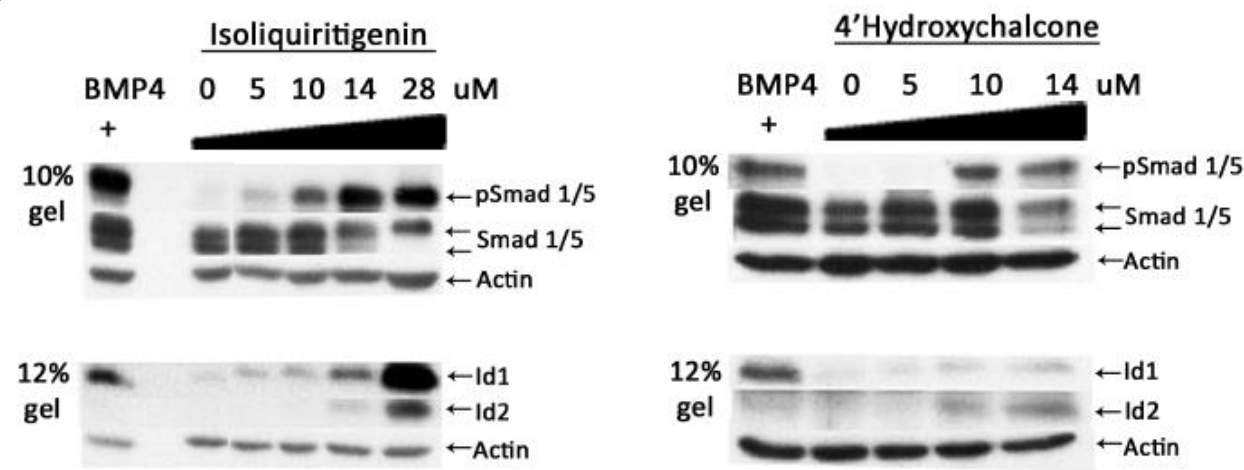

Figure 8: Small molecule activators of BMP signaling.

(A) Chemical structure of isoliquiritigenin (left) and 4'-hydroxychalcone (right). (B) Immunoblotting showing the activation of BMP signaling using antibodies for phosphorylated Smad 1/5, total Smad 1/5, Id1 and Id2 upon treating cells with increasing concentrations of isoliquiritigenin (left) and 4'-hydroxychalcone (right). Actin was used as a loading control. Id: inhibitor of differentiation; BMP4: bone morphogenetic protein 4; SMAD1/5: Small body size Mothers Against Decapentaplegic 1/5; pSMAD1/5: phosphorylated SMAD1/5. Image taken from Vrijens et al. (2013).

Chalcones or (E)-1,3-diphenyl-2-propen-1-ones are open chain flavonoids ubiquitously present in the plant kingdom. Due to their high abundance, easy synthesis and diverse bioactivities, 
chalcones are at a continuously growing interest for medicinal chemists. The $\alpha, \beta$-unsaturated ketone moiety allows the presence of cis and trans isomers of which the latter is more thermodynamically stable. It is also regarded as the main pharmacophore since studies have shown that bioactivity of chalcones is diminished with the full or partial loss of this moiety (Batovska and Todorova, 2010). However, it is also owing to the diverse phenyl substituents (such as hydroxyl, methoxy, alkyl, halogen, nitro-, amino-, etc.) that chalcones demonstrated a wide variety of bioactivities including anti-oxidant, anti-microbial, anti-cancer and antiinflammatory activities (Fig. 9).

In many cases, the same compound may have multiple biological properties. Isoliquiritigenin has recently shown to activate BMP signaling, but has also been used as an inhibitor of voltageoperated $\mathrm{K}^{+}$channels $\left(\mathrm{K}_{\mathrm{v}} 2.1\right)$ in rat cardiac myoblasts. This chalcone-induced positive inotropic response operated via increasing L-type calcium currents (Noguchi et al., 2008). Isoliquiritigenin can also inhibit the effects of cocaine by acting on the GABA $\mathrm{B}$ receptor (Jang et al., 2008) and exhibit anti-inflammatory activities (Batovska and Todorova, 2010; Zhou and Xing, 2015). The potential target profile of these chemotypes can present an obstacle to future clinical trials. However, their bioactivity in targeting signaling pathways (e.g. BMP) has yet to be comprehensively tested. 


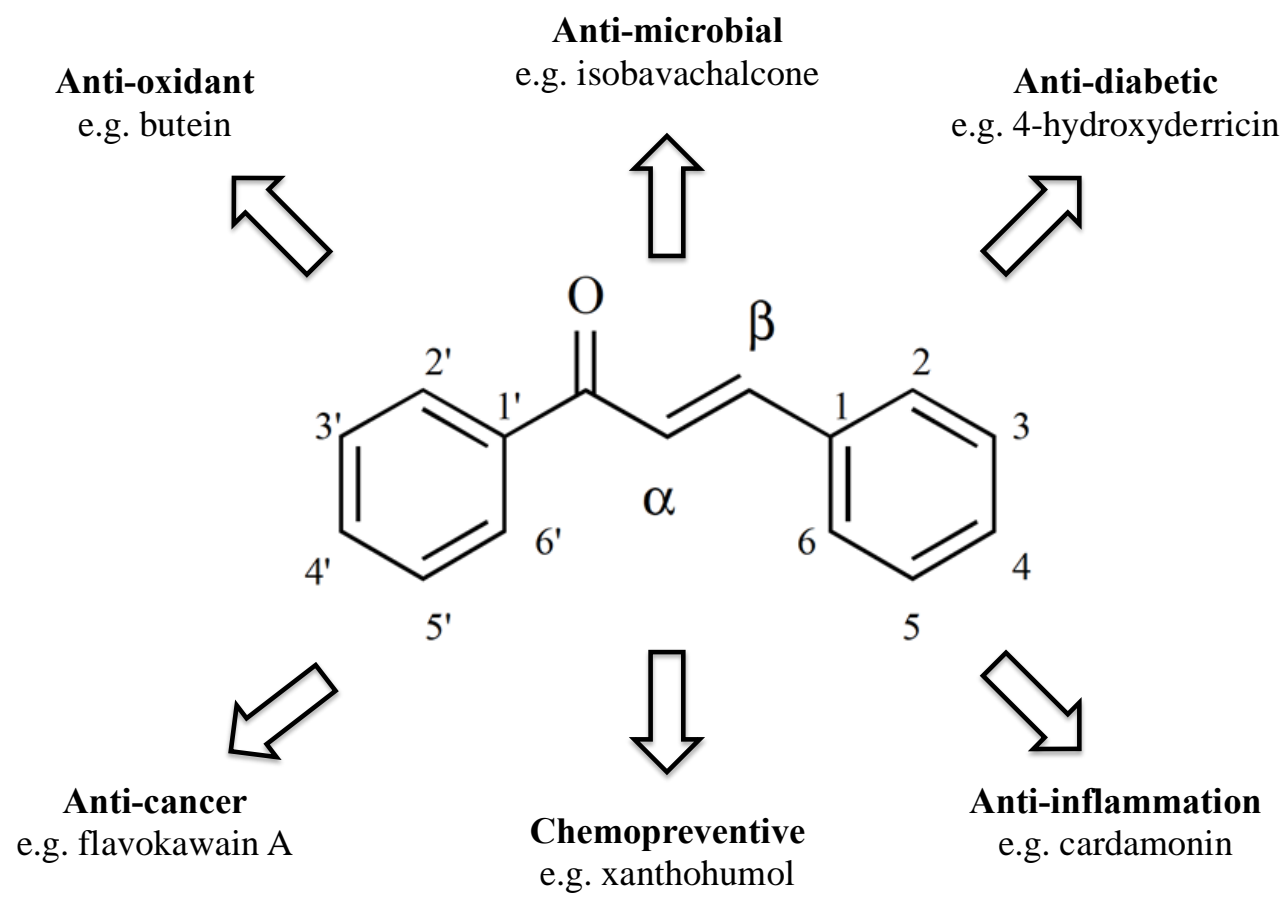

Figure 9: Backbone of the chalcone family and examples of biological activities.

Image adapted from Batavska and Todorova (2010). 


\subsection{Thesis Objectives and Hypotheses}

Building on the proof-of-concept for the directed self-organization of hPSC into cardiac organoids, introduced as bioengineered heart muscle (BHM) by our lab (Hudson et al., in revision), the objectives of this thesis entailed: (1: Chapter 3.1) a thorough characterization of the BHM model at the morphological, functional, and molecular level; (2: Chapter 3.2) the assessment of conditions that would allow for enhanced tissue maturation; (3: Chapter 3.3) identification of small molecule BMP4 mimics as a first step towards the development of protein-free directed differentiation and self-assembly of hPSCs into cardiac organoids. Accordingly, the following specific hypotheses were investigated:

1. BHM can be used to simulate human heart development.

2. Further functional maturation of BHM can be induced in long-term cultures.

3. Chalcones can be used to induce mesoderm formation in hPSCs.

A schematic outline of the working plan is depicted in Figure 10. 


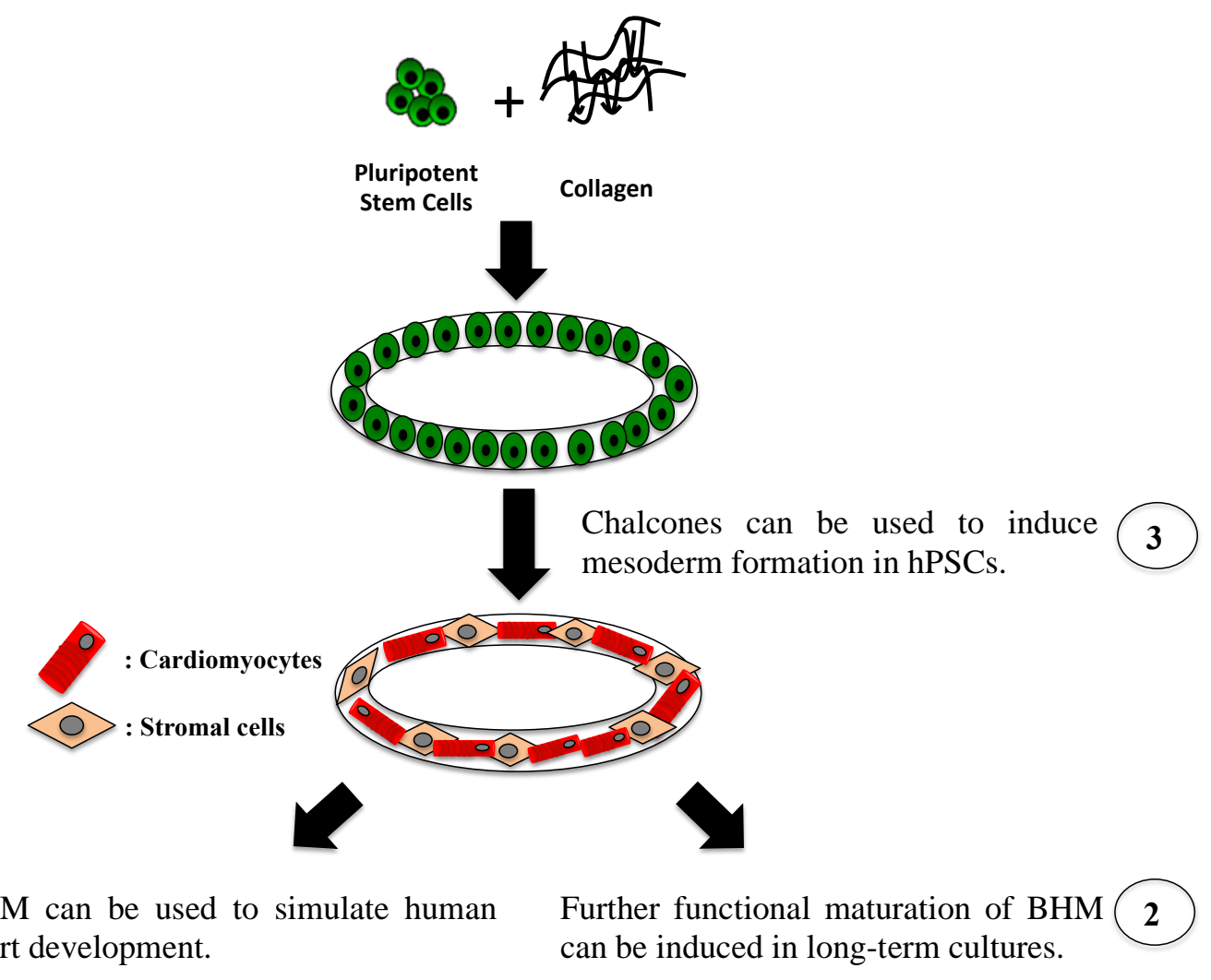

(1) BHM can be used to simulate human
heart development.

Figure 10: Schematic overview of the work program. 


\section{Materials and Methods}

\subsection{Cell Culture}

The import and experimentation with human embryonic stem cells (hESCs) was approved by the Robert-Koch-Institute (Az 1710-79-1-4-16-E1/2).

\subsubsection{Human feeder cell preparation}

Human foreskin fibroblasts (HFF) were cultured in fibroblast medium (Dulbecco's modified eagle medium (DMEM; Invitrogen) containing 20\% of fetal bovine serum (FBS; PAA) and 100 $\mathrm{IU} / \mathrm{mL}$ penicillin and $100 \mu \mathrm{g} / \mathrm{mL}$ streptomycin ([P/S]; Invitrogen) supplemented with $20 \mathrm{ng} / \mathrm{mL}$ of fibroblast growth factor-2 (FGF-2; Miltenyi Biotech). Irradiated HFFs (IR-HFFs) were obtained by exposing HFFs to 30 Gray $\gamma$-irradiation (STS Biobeam 8000, Germany) and subsequently plated down on a cell culture dish for further use in hESC maintenance (2.500 cells $/ \mathrm{cm}^{2}$ ).

\subsubsection{Human embryonic stem cell culture}

hESC cultures of HES2 cell line (originally obtained from ES-International, Singapore) were maintained on IR-HFF layers in HES medium (Dulbecco's modified eagle medium (DMEM; Invitrogen) containing $20 \%$ of Knock-out serum replacement (KOSR; Invitrogen) and FGF-2 was added at $10 \mathrm{ng} / \mathrm{mL}$. Passaging with TrypLE Express ( $3 \mathrm{~mL} / \mathrm{flask}$; Invitrogen) was performed at day 7 followed by splitting over several passages onto IR-HFFs to obtain single cell suspensions ready to be transferred to feeder-free conditions. This involved cells passaged and plated at $25.000 \mathrm{cells} / \mathrm{cm}^{2}$ in maintenance medium (HES and IR-HFF conditioned medium [1:1] supplemented with $20 \mathrm{ng} / \mathrm{ml} \mathrm{FGF-2)} \mathrm{for} \mathrm{three} \mathrm{days} \mathrm{on} \mathrm{plates} \mathrm{precoated} \mathrm{for} \mathrm{60-90} \mathrm{minutes}$ with Matrigel (BD Bioscience, 1:30 in phosphate buffered saline (PBS). The aforementioned step was done prior to initiation of differentiation (day-4). IR-HFF cultures $\left(2.000 \mathrm{cells} / \mathrm{cm}^{2}\right)$ were maintained in HES medium for 7 days and medium was collected every 48 hours (IR-HFF 
conditioned medium). hESC cultures were regularly tested for mycoplasma (Lonza Mycoalert ${ }^{\mathrm{TM}}$ Kit).

\subsection{D Cardiac Differentiation}

\subsubsection{Differentiation medium}

hESCs were cultured in basal medium: Rosewell Park Memorial Institute (RPMI 1640) supplemented with P/S, $1 \mathrm{mmol} / \mathrm{L}$ sodium pyruvate and 2\% B27 serum replacement supplement with insulin (all Gibco) in the presence of L-ascorbic acid 2 phosphate sesquimagnesium salt hydrate (ASC; Sigma; $200 \mu \mathrm{mol} / \mathrm{L}$ ). Factors were added to the medium as indicated in (Fig. 11).

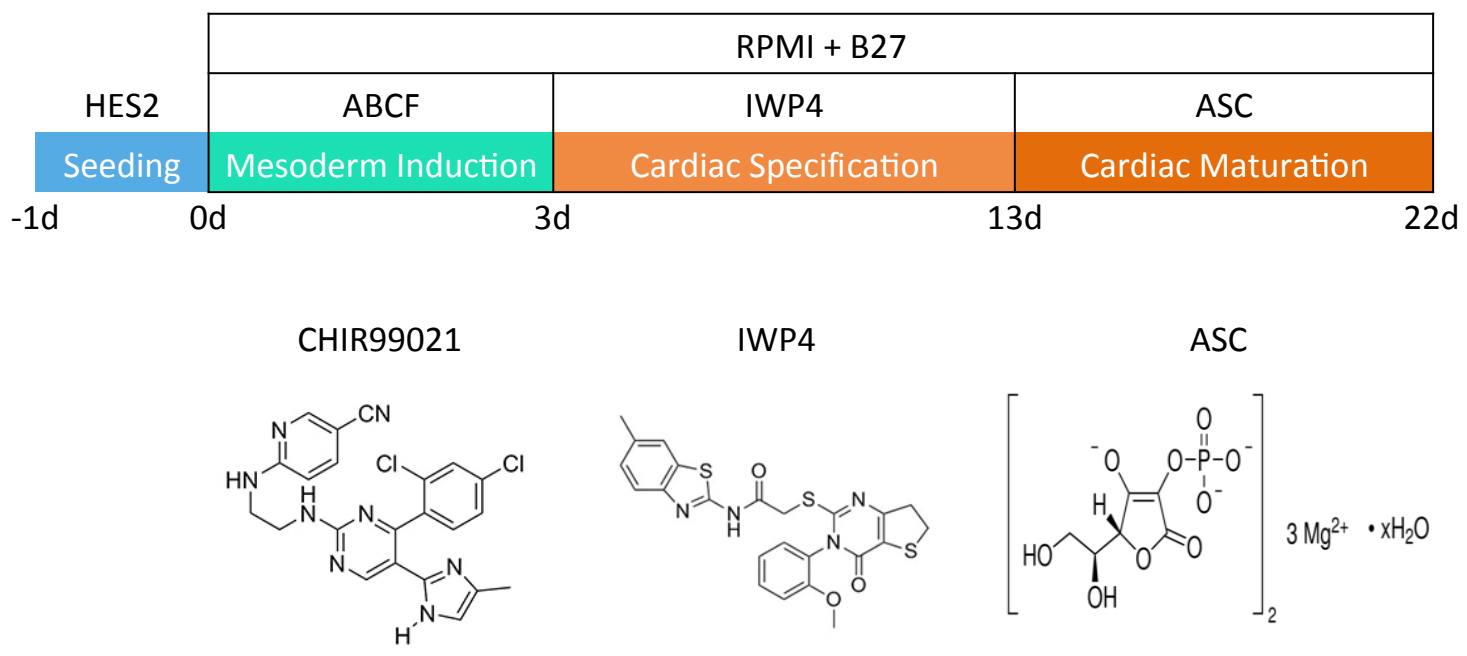

Figure 11: Outline of the cardiac differentiation of HES2 cells in monolayer cultures.

HES2: human embryonic stem cell line 2, A: activin-A, B: bone morphogenetic protein 4, C: CHIR99021, F: fibroblast growth factor-2, IWP4: inhibitor of Wnt production 4, ASC: L-ascorbic acid, RPMI: Rosewell Park Memorial Institute medium, B27: defined culture medium supplement, d: days. Image adapted from Hudson et al., in revision. 


\subsubsection{Monolayer differentiation}

At day -1 cells were passaged and plated at 50.000 cells $/ \mathrm{cm}^{2}$ on Matrigel ${ }^{\mathrm{TM}}$ coated multi-well plates in maintenance medium. 24 hours later, cells were washed with basal medium first $(0.5$ $\mathrm{mL} /$ well in a 24-well plate) before the start of the mesoderm induction phase (3 days); following growth factors were added to the basal medium for mesoderm induction: CHIR99021 (Stemgent; $1 \mu \mathrm{mol} / \mathrm{L}$ ), FGF-2 ( $5 \mathrm{ng} / \mathrm{mL}$ ), BMP4 (R\&D systems; $5 \mathrm{ng} / \mathrm{mL}$ ), Activin-A (R\&D systems; $9 \mathrm{ng} / \mathrm{mL}$ ). Culture medium was exchanged daily until day 3 (3 medium changes). Subesquently, cardiac specification was induced for 10 days with the addition of an inhibitor of Wnt production (IWP4; Stemgent; $5 \mu \mathrm{mol} / \mathrm{L}$ ) to the basal medium, which was exchanged every 2-3 days until day 13 (4 medium changes). Thereafter, cardiomyocytes were maintained in basal medium with medium exchanges every 2 days (4 medium changes). Beating was observed starting day 13 and the cells were harvested on day 22 .

\subsubsection{Dissociation of differentiated hESC}

Cells were rinsed with PBS first, followed by incubation for 15 minutes in Accutase solution (Millipore) supplemented with $0.025 \%$ Trypsin (2.5\%; Gibco) and 2\% DNase I (1 mg/mL stock solution; Calbiochem) at room temperature $(0.5 \mathrm{~mL} /$ well $)$. The cells were then collected in a tube, rinsed with 5\% FBS in PBS and pelleted at $300 \mathrm{x}$ g for 4 minutes. Following aspiration of the supernatant, the dissociated cells were then fixed in ethanol (70\%) for flow cytometry.

\subsection{Bioengineered Heart Muscle Generation}

\subsubsection{Preparation of casting molds and dynamic stretchers}

For the generation of BHMs, circular molds of inner/outer diameter $4 / 10 \mathrm{~mm}$ were fabricated using poly-dimethylsiloxane (PDMS; Sylgard, Dow Corning) and allowed to harden overnight at $55^{\circ} \mathrm{C}$ (Fig. 12A). The detailed protocol for the preparation of the casting molds has been described before (Soong et al., 2012; Tiburcy et al., 2014). Similarly, silicone based flexible stretchers (length: $12 \mathrm{~mm}$, thickness: $1.5 \mathrm{~mm}$, inner/outer distance: $6 / 7.5 \mathrm{~mm}$ ) were generated 
and used to facilitate auxotonic contractions of BHMs (Fig. 12B). Both the assembled casting molds and the flexible stretchers can be autoclaved and reused.

A

(i)

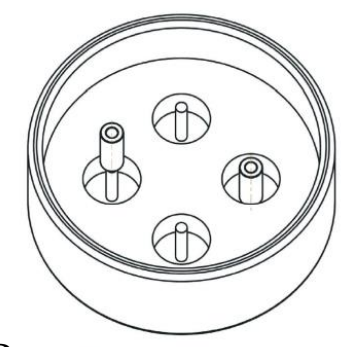

B (ii)

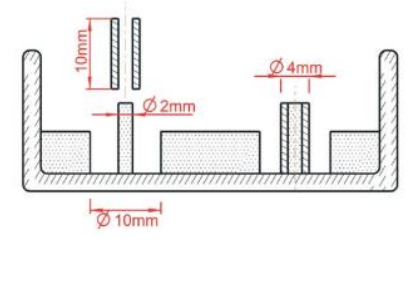

(ii)

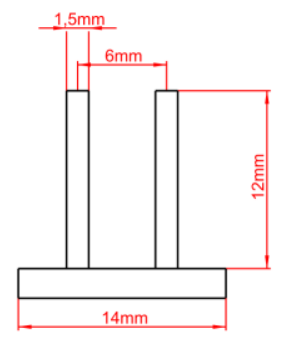

(iii)

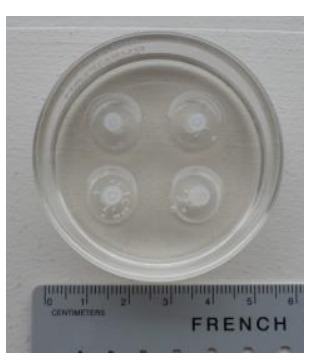

(iii)

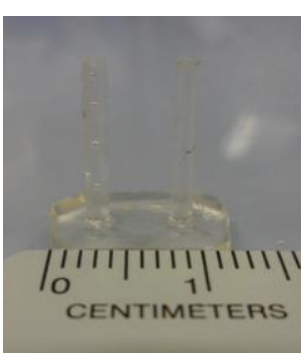

Figure 12: Casting mold and silicone poles for BHM generation and loading.

(A) Glass culture dish filled with transparent silicone and 4 circular recesses with central spacers. Each circular recesses $($ diameter $=10 \mathrm{~mm})$ has a central silicone core $($ diameter $=2 \mathrm{~mm})$ which has a thin silicone tubing (length= $10 \mathrm{~mm}$ ) placed over it to provide an inner spacer during BHM generation. (B) Dynamic silicone poles (length= 12 $\mathrm{mm}$, thickness $=1.5 \mathrm{~mm}$, inner/outer distance $=6 / 7.5 \mathrm{~mm}$ ). Images adapted from Tiburcy et al. $(2014)$.

\subsubsection{Bioengineered heart muscle assembly and differentiation}

BHMs were prepared from HES2 cells maintained for 3 days in feeder-free conditions (see section 2.1.2). At day -1 cells were dissociated into single cells using TrypLE and $5 \times 10^{5}$ HES2 cells suspended in maintenance medium were mixed with the BHM reconstitution mixture composed of 1:1 v/v mix of acid solubilized bovine collagen I (LLC collagen solutions) and 2X DMEM (Gibco) neutralized by titration with $0.1 \mathrm{~mol} / \mathrm{L}$ sodium hydroxide. The final collagen I concentration was $1 \mathrm{mg} / \mathrm{mL}$. $170 \mu \mathrm{l}$ of the BHM reconstitution mixture was cast into the molds and allowed to consolidate for one hour at $37^{\circ} \mathrm{C}$ after which maintenance medium (1.25 
mL/BHM) was added (Fig. 13). 24 hours later, BHMs were first rinsed with RPMI medium then induced to differentiate to cardiac lineage with temporal addition of growth factors and small molecules as outlined in the schematic below. At day 13, BHMs were transferred on to dynamic stretchers and cultured for 9 days in basal medium with adjusted calcium concentration from 0.4 $\mathrm{mmol} / \mathrm{L}$ to physiological concentration of $1.2 \mathrm{mmol} / \mathrm{L}$. TGF $\beta-1(1 \mathrm{ng} / \mathrm{mL})$ or other factors were screened for further BHM maturation. Analysis and characterization of BHMs were performed at day 22 unless indicated otherwise.

\begin{tabular}{|c|c|c|c|}
\hline \multirow[b]{3}{*}{ HES2 } & \multicolumn{3}{|c|}{$\mathrm{RPMI}+\mathrm{B} 27+\mathrm{ASC}$} \\
\hline & \multicolumn{2}{|c|}{$0.4 \mathrm{mmol} / \mathrm{L}$ Calcium } & $1.2 \mathrm{mmol} / \mathrm{L}$ Calcium \\
\hline & $\mathrm{ABCF}$ & IWP4 & Dynamic Stretching + TGF $\beta-1$ \\
\hline Casting & Mesoderm Induction & Cardiac Specification & Cardiac Maturation \\
\hline$\frac{-1 d}{(i)}$ & & & $22 c$ \\
\hline
\end{tabular}

Figure 13: Outline of HES2-BHM generation and differentiation.

HES2 cells were mixed with collagen type I and cast (i) into empty circular molds. (ii) After 24 hours the tissue constructs condensed and exposed to sequential addition of growth factors and small molecules to enhance mesoderm induction, cardiac specification and cardiac maturation. BHMs are subjected to (iii) mechanical loading at day 13 and cultured further till day 22. HES2: human embryonic stem cell line 2; A: activin-A; B: bone morphogenetic protein 4; C: CHIR99021; F: fibroblast growth factor-2; IWP4: inhibitor of Wnt production 4; TGF $\beta-1$ : transforming growth factor $\beta-1$; RPMI: Rosewell Park Memorial Institute basal medium; B27: defined culture medium supplement; ASC: ascorbic acid; d: days. Image adapted from Hudson et al. in revision.

\subsubsection{Bioengineered heart muscle dissociation}

Collagenase ( $0.2 \%$; Sigma) prepared in PBS (with calcium/magnesium) with $20 \%$ fetal bovine serum (FBS; Gibco) was added (1 mL/BHM) for $60-90$ minutes at $37^{\circ} \mathrm{C}$ after which the supernatant was collected in a falcon tube $(15 \mathrm{~mL})$ and the remaining large pieces from each digested BHM were resuspended in $1 \mathrm{~mL}$ of Accutase solution (Millipore) supplemented with 0.025\% Trypsin (2.5\%; Gibco) and 2\% DNase I (1 mg/mL stock solution; Calbiochem) for 30 minutes at room temperature. To stop the digestion, 5\% FBS in PBS (1 mL/BHM) was added 
and the whole mixture was pipetted and transferred to the same falcon tube. Single cells obtained from the dissociated BHMs were then either fixed in ethanol (70\%) for staining of intracellular markers or formaldehyde solution (4\%; Roth) for subsequent labelling of cell surface markers.

\subsection{Analysis}

\subsubsection{Isometric force measurement}

BHMs were suspended in organ baths at $37^{\circ} \mathrm{C}$ in gassed $\left(5 \% \mathrm{CO}_{2} / 95 \% \mathrm{O}_{2}\right)$ Tyrode's solution (mmol/L: $\mathrm{NaCl} 126.7, \mathrm{KCl} 5.4, \mathrm{CaCl}_{2}$ 0.2, $\mathrm{MgCl}_{2}$ 1.05, $\mathrm{NaHCO}_{3} 22, \mathrm{NaH}_{2} \mathrm{PO}_{4}$ 0.42, glucose 5.6, ascorbic acid 0.56; $\mathrm{pH}$ 7.4). Calcium concentration was adjusted using $\mathrm{CaCl}_{2}$ solution (0.2 $\mathrm{mol} / \mathrm{L})$ diluted from stock solution $(2.25 \mathrm{~mol} / \mathrm{L})$. After calibration and reaching equilibrium, BHMs were stimulated with electric field pulses ( $2 \mathrm{~Hz}, 5 \mathrm{~ms}, 200 \mathrm{~mA}$ ) at $2 \mathrm{mmol} / \mathrm{L}$ calcium and stretched stepwise by $125 \mu \mathrm{m}$ until the twitch tension reached a maximum value according to the Frank-Starling mechanism (this encompassed typically 4 x $125 \mu \mathrm{m}$ stretches). All subsequent analyses were performed at $\mathrm{L}_{\max }$, defined as the tissue length with maximal detected force of contraction (twitch amplitude). The maximal inotropic capacity was assessed by investigating the inotropic response to cumulatively increasing calcium concentrations (0.2-4 $\mathrm{mmol} / \mathrm{L}$ unless indicated otherwise). Response to $\beta$-adrenergic stimulation using Isoprenaline (1 $\mu \mathrm{mol} / \mathrm{L})$ was investigated at $\mathrm{EC}_{50}$ calcium concentration.

\subsubsection{Action potential recordings}

With the help of Dr. Mei-Ling Liao (Institute of Pharmacology and Toxicology), impaling electrode measurements on the BHMs were performed. BHMs were pinned down in a customized chamber and perfused with Tyrode's solution $\left(1 \mathrm{~mL} / \mathrm{minute}, 35-37^{\circ} \mathrm{C}\right.$; mmol/L: $\mathrm{NaCl} 150, \mathrm{KCl} 5.4, \mathrm{MgCl}_{2} 2, \mathrm{CaCl}_{2}$ 1.8, HEPES 10, glucose 10; pH 7.4; $100 \% \mathrm{O}_{2}$ ). Microelectrodes with 30-50 $\mathrm{M} \Omega$ tip resistance were filled with $2 \mathrm{~mol} / \mathrm{L} \mathrm{KCl}$ and used to impale cardiomyocytes in BHM. Signals were acquired (LIH 8+8 Interface, HEKA) and amplified (SEC-05X, NPI). Data analysis was performed with LabChart software (ADInstruments). 
Cardiomyocyte action potentials can be grossly subdivided into action potentials typically observed in ventricular and pacemaker myocytes (Fig. 14A). The differences in action potential kinetics are determined by the expression and phase specific activation of specific ion channels and currents (Grant, 2009) (Fig. 14 B and C). 
A<smiles>C1CCCCC1</smiles>
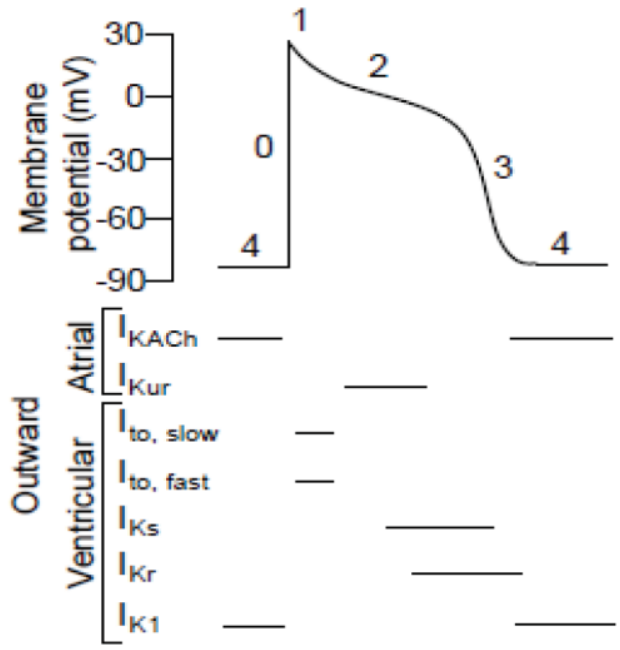

B

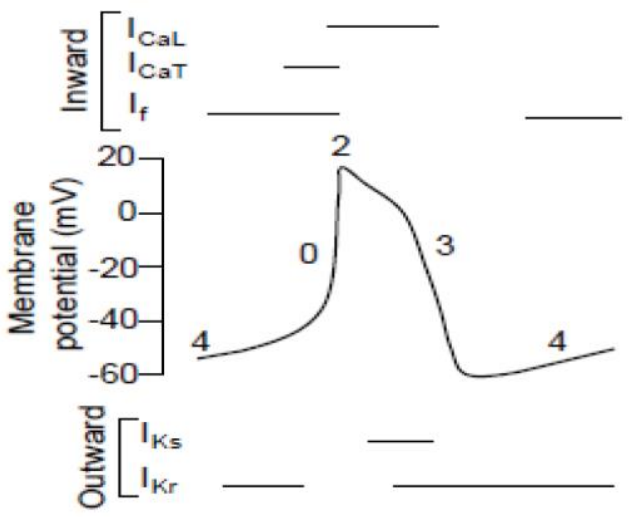

$\mathrm{C}$

\begin{tabular}{|c|c|c|c|c|c|}
\hline Current & Description & AP Phase & Activation Mechanism & Clone & Gene \\
\hline \multicolumn{6}{|c|}{$\alpha$-subunit of action potential inward current channels } \\
\hline ha & Sodium current & Phase 0 & Voltage, depolarization & $\mathrm{Na}_{\mathrm{v}} 1.5$ & SCN5A \\
\hline$I_{\mathrm{Ca}, \mathrm{L}}$ & Calcium current, L-type & Phase 2 & Voltage, depolarization & $\mathrm{Ca}_{\mathbf{y}} 1.2$ & CACNA1C \\
\hline $\mathrm{ICa}, \mathrm{T}_{\mathrm{T}}$ & Calcium current, T-type & Phase 2 & Voltage, depolarization & $\mathrm{Ca}_{\mathrm{g}} 3.1 / 3.2$ & CACNA1G \\
\hline \multicolumn{6}{|c|}{$\alpha$-subunit of action potential outward $\left(\mathrm{K}^{+}\right)$current channels } \\
\hline Ito,f & Transient outward current, fast & Phase 1 & Voltage, depolarization & KV $4.2 / 4.3$ & $\mathrm{KCND} 2 / 3$ \\
\hline \multirow[t]{3}{*}{ Ito,s } & Transient outward current, slow & Phase 1 & Voltage, depolarization & KV $1.4 / 1.7 / 3.4$ & KCNA4 \\
\hline & & & & & KCNA7 \\
\hline & & & & & KCNC4 \\
\hline \multirow[t]{2}{*}{$l_{\text {Kur }}$} & Delayed rectifier, ultrarapid & Phase 1 & Voltage, depolarization & KV $1.5 / 3.1$ & KCNA5 \\
\hline & & & & & KCNC1 \\
\hline$l_{\mathrm{kr}}$ & Delayed rectifier, fast & Phase 3 & Voltage, depolarization & HERG & KCNH2 \\
\hline$l_{\mathrm{ks}}$ & Delayed rectifier, slow & Phase 3 & Voltage, depolarization & KVLOT1 & KCNQ1 \\
\hline$l_{\mathrm{K} 1}$ & Inward rectifier & Phase $3 \& 4$ & Voltage, depolarization & Kir $2.1 / 2.2$ & KCNJ2/12 \\
\hline$l_{\text {KATP }}$ & ADP activated $\mathrm{K}+$ current & Phase $1 \& 2$ & {$[\mathrm{ADP}] /[\mathrm{ATP}] \uparrow$} & Kir 6.2 (SURA) & KCNJ11 \\
\hline$l_{\text {KACh }}$ & Muscarinic-gated $\mathrm{K}+$ current & Phase 4 & Acetylcholine & Kir $3.1 / 3.4$ & $\mathrm{KCNJ} 3 / 5$ \\
\hline \multirow[t]{3}{*}{$l_{\mathrm{KP}}$} & Background current & All Phases & Metabolism, stretch & TWK-1/2 & KCNK1/6 \\
\hline & & & & TASK-1 & KCNK3 \\
\hline & & & & TRAAK & KCNK4 \\
\hline I & Pacemaker current & Phase 4 & Voltage, hyperpolarization & $\mathrm{HCN} 2 / 4$ & $\mathrm{HCN} 2 / 4$ \\
\hline
\end{tabular}

Figure 14: Action potential traces and membrance currents from ventricular and pacemaker cells. Image adapted from Hudson et al. in revision and Grant (2009). 


\subsubsection{Flow cytometry}

Cells dissociated from 2D/3D differentiation procedures and fixed with ethanol or $4 \%$ formaldehyde solution were resuspended in blocking buffer (PBS supplemented with 5\% FBS, $1 \%$ bovine serum albumin [BSA], $0.5 \%$ Triton $\mathrm{X}-100$ and sodium azide; used for internal markers) or 5\% FBS in PBS (for cell surface markers) and permeabilized for 10 minutes at $4^{\circ} \mathrm{C}$ then filtered using a cell strainer $(40 \mu \mathrm{m})$. Samples were incubated for 45 minutes at $4^{\circ} \mathrm{C}$ with primary antibodies (Table 4, Appendix), followed by two washing steps with blocking buffer prior to incubation with secondary antibodies in blocking buffer (Table 4, Appendix) and Hoechst 33342 for 30 minutes at $4^{\circ} \mathrm{C}$. Samples were then again washed with blocking buffer and resuspended in PBS for analysis with a BD LSRII flow cytometer (BS Biosystems). Viable cells were first gated based on Hoechst 33342 staining. The gating strategy for $\alpha$-actinin positive cells is shown in (Fig. 15); single cells were gated based on forward scatter signal, IgG controls were used to determine non-specific staining. BD FACSDiva Software (BD Biosciences) or Cyflogic Software were used for analysis.

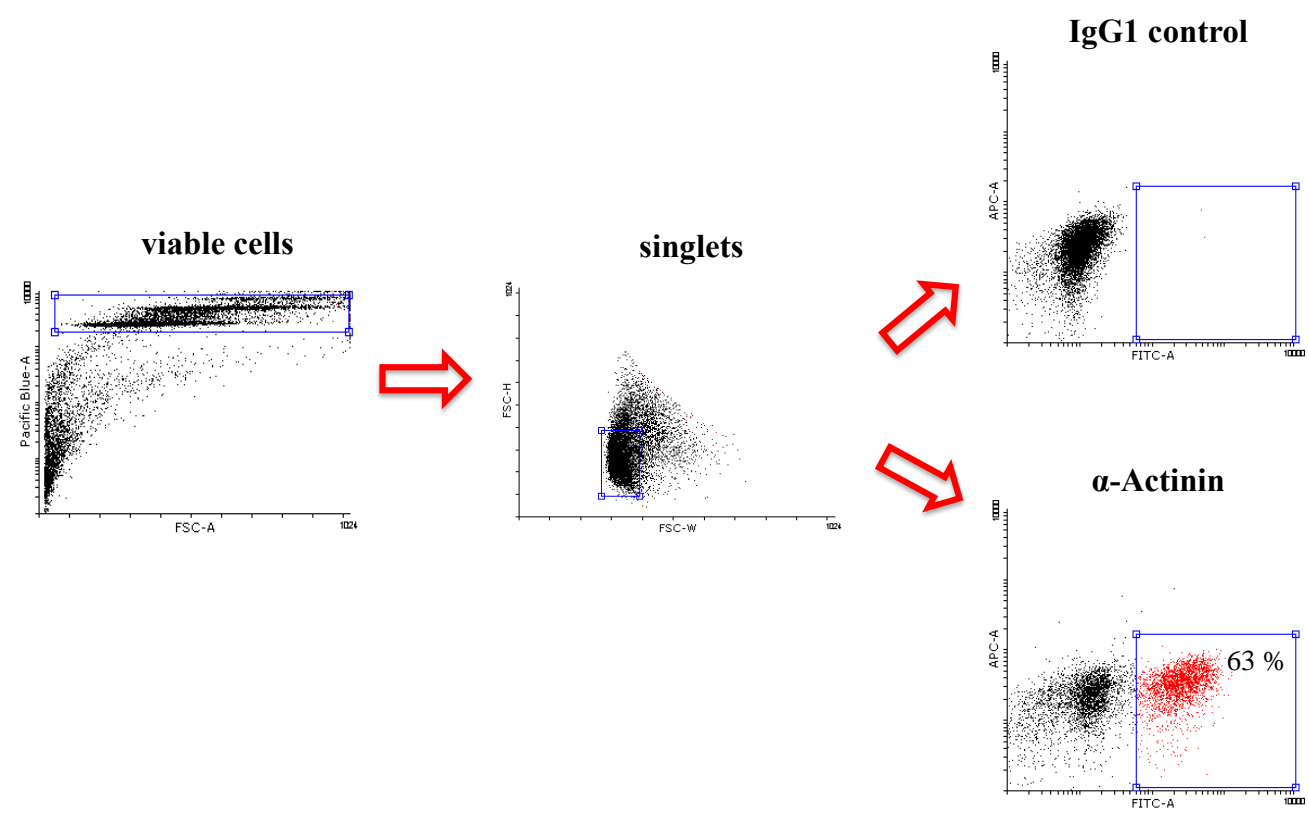

Figure 15: Gating strategy for $\alpha$-actinin ${ }^{+}$cardiomyocytes.

Viable cells in G1 + G2 were selected based on Hoechst3342 intensity. Doublets were excluded based on forward scatter width (FSC-W). Cardiomyocytes were distinguished based on actinin positivity. IgG1 was used as an isotype control for non-specific staining. 


\subsubsection{Quantification of cardiomyocyte size using flow cytometry}

Cardiomyocyte size in the different experimental conditions was assessed using the median side scatter intensity (SSC) in the gated $\alpha$-actinin positive cell population.

\subsubsection{Whole mount staining and confocal imaging}

To visualize BHM morphology and cellular composition, whole mount staining and imaging were performed. Prior to immunostaining, BHMs were fixed with formaldehyde solution (4\%; overnight, $4^{\circ} \mathrm{C}$ ). Samples were then permeabilized with $0.005 \%$ Triton X-100 for one hour at room temperature followed by three washing steps with PBS. Samples were blocked with Rotiblock ${ }^{\mathrm{TM}}$ (Roth) overnight at $4^{\circ} \mathrm{C}$ and subsequently incubated at $4^{\circ} \mathrm{C}$ with primary antibodies (Table 4, Appendix) in PBS with constant rotation for two nights. Prior to incubation with secondary antibodies for two nights at $4{ }^{\circ} \mathrm{C}$ in PBS, samples were washed 3 times in PBS. Subsequent washing with PBS proceeded mounting the BHMs with fluoromont (Southern Biotech) on microscope slides covered with a coverslip to compress BHM equally. Fluorescence was then visualized with a Zeiss 710 NLO confocal microscope.

\subsubsection{Transmission electron microscopy}

TEM measurements were performed in collaboration with Wiebke Moebius at the Max-PlanckInstitute for Experimental Medicine. The detailed procedure has been described before (Zimmermann et al., 2002). In short, BHM sections (1x2 mm) were fixed in $2.5 \%$ glutaraldehyde in PBS containing $1 \mathrm{mmol} / \mathrm{L} \mathrm{CaCl}_{2}$ and $30 \mathrm{mmol} / \mathrm{L}$ 2,3-Butanedione-2monoxime $(\mathrm{BDM})$ overnight at $4^{\circ} \mathrm{C}$. After an overnight wash in $0.1 \mathrm{~mol} / \mathrm{L}$ phosphate buffer $\mathrm{pH}$ 7.3 samples were post-fixed in $2 \% \mathrm{OsO}_{4}$ (Science Services) and $3 \%$ potassium ferricyanide (1:1) in $0.1 \mathrm{~mol} / \mathrm{L}$ phosphate buffer $\mathrm{pH} 7.3$ for 4 hours at $4^{\circ} \mathrm{C}$ and embedded in EPON (SERVA Electrophoresis $\mathrm{GmbH}$ ) after dehydration with ethanol and propylenoxide. Ultrathin sections of

$50 \mathrm{~nm}$ thickness were prepared with a Ultracut S ultramicrotome (Leica), stained with an aqueous solution of $4 \%$ uranyl acetate followed by lead citrate (Reynolds, 1963). Pictures were 
taken with a LEO EM912 omega electron microscope (Zeiss) using an on-axis 2k CCD camera (TRS, Moorenweis, Germany).

\subsubsection{Gene expression analysis}

\subsubsection{RNA preparation}

Total RNA was isolated from cells and BHM using the Trizol ${ }^{\mathrm{TM}}$ (Invitrogen) standard extraction protocol. Cells/BHMs were first washed in PBS. Subsequently, $1 \mathrm{~mL}$ of Trizol reagent was added for 5 minutes at room temperature. Cells were then scratched off the culture plates using cell scrapers and the supernatant was collected. BHMs were lysed using Tissue Lyser II (Qiagen) at $25 \mathrm{~Hz}$ for 5 minutes at $4^{\circ} \mathrm{C}$ in the presence of $7 \mathrm{~mm}$ stainless steel beads (Qiagen). Chloroform (200 $\mu \mathrm{L} /$ sample) was then added, followed by thorough vortexing. After incubation for 10 minutes at room temperature, phase-separation was facilitated by centrifuging at $12.000 \mathrm{x}$ $\mathrm{g}$ and $4^{\circ} \mathrm{C}$ for 10 minutes. The upper aqueous phase was transferred to RNase-free reaction tube ( $2 \mathrm{~mL}$; Eppendorf). The RNA was pelleted by incubation in isopropanol (500 $\mu \mathrm{L} / \mathrm{sample}$ ) for 10 minutes at room temperature and centrifuging at $12,000 \times \mathrm{g}$ (at $4^{\circ} \mathrm{C}$ for 10 minutes). Following removal of the supernatant, the RNA pellet was washed with $75 \%$ ethanol $(1 \mathrm{~mL} / \mathrm{sample})$ and the tubes were spun at $12,000 \mathrm{xg}$ (at $4^{\circ} \mathrm{C}$ for 5 minutes). The RNA pellet was air-dried for 10 minutes and resuspended in DEPC water (25 $\mu \mathrm{L} /$ sample, Sigma). RNA concentration was quantified using the Nanodrop spectrophotometer (Thermo Scientific) and samples were stored at $-80^{\circ} \mathrm{C}$. RNA samples had an average of $260 / 280$ ratio of 1.8 .

\subsubsection{2 cDNA synthesis}

To remove potential genomic DNA contamination, RNA samples were treated with DNase I (Roche) as follows; $0.2 \mu \mathrm{L}$ of DNase I (10 U/ $\mu \mathrm{L})$ was added to $1 \mu \mathrm{g}$ of RNA mixed with $1 \mu \mathrm{L}$ of DNase incubation buffer. The mix was then incubated for 20 minutes at $37^{\circ} \mathrm{C}$. To inactivate the enzyme, $1 \mu \mathrm{L}$ of EDTA $(0.2 \mathrm{mmol} / \mathrm{L}, \mathrm{pH} 8)$ was added to the mix which was incubated further for 10 minutes at $75^{\circ} \mathrm{C}$. The samples were further used for complementary DNA (cDNA) synthesis. 
High Capacity cDNA Reverse Transcription Kit (Applied Biosystems) was used for reverse transcription according to manufacturer's instructions. For one reverse transcription (RT) reaction, $25 \mathrm{X}$ dNTPs $(0.8 \mu \mathrm{L})$ were mixed with $10 \mathrm{X}$ RT random primers $(2 \mu \mathrm{L})$ in 10X RT buffer $(2 \mu \mathrm{L})$ and DEPC treated water $(2.2 \mu \mathrm{L})$. RNase inhibitor $(1 \mu \mathrm{L})$ was added to the master mix from which $8 \mu \mathrm{L}$ was transferred to each DNase treated samples. To control for unwanted genomic DNA amplification, a negative control from each condition was processed in which

RNase free water $(1 \mu \mathrm{L})$ replaced the MultiScribe ${ }^{\mathrm{TM}}$ Reverse Transcriptase. The samples were then transferred to the $\mathrm{T}$ gradient thermocycler (Biometra) and the following program was run $\left(25^{\circ} \mathrm{C}\right.$ for 10 minutes, $37^{\circ} \mathrm{C}$ for 120 minutes, $85^{\circ} \mathrm{C}$ for 5 minutes then paused at $4^{\circ} \mathrm{C}$ ). After cDNA synthesis, the samples were stored at $-20^{\circ} \mathrm{C}$ to be used later for semiquantitative or quantitative polymerase chain reaction (PCR).

\subsubsection{Quantitative RT-PCR ( $q P C R)$}

qPCR was done using Fast SYBR Green Master Mix (PCR Biosystems) on a 384-well format AB7900 HT Fast Real-Time PCR system (Applied Biosystems). For product specificity no template and no reverse transcriptase served as control. Expression profile was analyzed using the standard curve of gene of interest with known concentration $\left(10^{3}-10^{8}\right.$ molecules $\left./ \mu \mathrm{L}\right)$ by SDS2.4 software. Glyceraldehyde 3-phosphate dehydrogenase (GAPDH) was used as a house keeping gene for normalization. Primer details are listed in the Appendix.

\subsubsection{RNA sequencing and bioinformatics analyses}

RNA sequencing was performed at the Microarray and Deep-Sequencing Facility of the University Medical Center Goettingen (head: Dr. Gabriela Salinas-Reisters). As described above, total RNA was isolated and integrity was assessed with the Agilent Bioanalyzer 2100. Only samples exhibiting a RNA integrity number (RIN) of $>7$ were subjected to RNA sequencing. Total RNA ( $n=4$ from 3 independent experimental series for each investigated timepoint) was subjected to library preparation (TruSeq Stranded Total RNA Sample Prep Kit from Illumina) and RNA-sequencing on an Illumina HighSeq-2000 platform (SR 50 bp; >25 Mio reads/sample). With the help from our collaborators at the Institute of Cardiovascular 
Regeneration in Goethe-University Frankfurt (Dr. Shizuka Uchida), the raw data (FASTQ) were mapped to GRCh38/hg38 using TopHat2 (Kim et al., 2013) and analyzed by Cufflinks (Trapnell et al., 2012) to calculate Fragments Per Kilobase of Exon per Million Fragments (FPKM) values. Data with the biotype "protein_coding" and FPKM $>1$ were considered for further analysis. Self-organizing tree algorithm (SOTA) (Dopazo and Carazo, 1997; Herrero et al., 2001) was assessed using MultiExperiment Viewer (MeV) (Saeed et al., 2003). Gene ontology (GO) and Kyoto Encyclopedia of Genes and Genomes (KEGG) pathway analysis was performed through DAVID (Huang da et al., 2009). All RNA-seq data sets have been deposited in the Gene Expression Omnibus.

\subsubsection{Western blot}

\subsubsection{Protein extraction from monolayer cells}

To obtain cell lysates from 2D cultures, HES2 cells were first rinsed with ice-cold PBS then scratched off from the culture dish using a cell scraper, collected in a $1.5 \mathrm{~mL}$ reaction tube (Eppendorf) and pelleted at 12,000 x g for 4 minutes. Next, $200 \mu \mathrm{L}$ of CytoBuster ${ }^{\mathrm{TM}}$ lysis buffer (Novagen) supplemented with phosphatase and protease inhibitors was added to the cell pellet and incubated at room temperature for 15 minutes while mixing thoroughly to homogenize. The supernatant was subsequently transferred to new polypropylene collection tubes (Eppendorf). The cell debris in samples from monolayer cultures was then pelleted at $12,000 \times \mathrm{g}$ for 20 minutes at $4^{\circ} \mathrm{C}$. The resulting supernatant was then referred to as protein lysate and transferred to a new collection tube (Eppendorf) and stored at $-20^{\circ} \mathrm{C}$ until further use.

\subsubsection{Western blotting}

Prior to Western Blotting, protein concentration from each sample was quantified using Bradford assay with BSA (Sigma-Aldrich) as a standard. Sodium dodecyl sulfate polyacrylamide gel electrophoresis (SDS-PAGE) was used to separate proteins from a protein lysate according to their molecular mass. Negatively charged SDS binds to proteins and leads to their repulsion of each other. The reagents used for the gel preparation are listed in the 
Appendix. Briefly, $15 \mu \mathrm{g}$ of protein lysates were loaded on a $6 \%$ collecting gel (Appendix) after mixing for 5 minutes at $95^{\circ} \mathrm{C}$ in $4 \times$ Laemmli buffer. Proteins were then resolved on a $10 \%$ separating gel (Appendix). Protein collection was done at $70 \mathrm{~V}$ for 20 minutes and seperated at $140 \mathrm{~V}$ for 50 minutes. Proteins were transferred from the PAGE to polyvinylidene fluoride (PVDF) membrane in a vertical electric field at $140 \mathrm{~mA}$ for one hour. Next, the membrane was blocked in 5\% BSA in TBST buffer (Tris-HCl [20 mmol/L, pH 7.6] containing 0.1\% Tween-20 [Tween ${ }^{\circledR} 20$, Sigma-Aldrich] and $\mathrm{NaCl}$ [165 mmol/L, Roth]) for one hour at room temperature then incubated overnight at $4{ }^{\circ} \mathrm{C}$ in primary antibody diluted in blocking buffer $(5 \%$ BSA in $1 \mathrm{x}$ TBST). Following three washing steps with TBST, the membrane was incubated with secondary antibody (also diluted in blocking buffer) for one hour at room temperature. The list of primary and secondary antibodies used in this study and their respective dilution factor are listed in the Appendix. The membrane was again washed three times with TBST prior to incubation with

horseradish peroxidase substrate (SuperSignal West Femto kit, Pearce, Thermo Scientific) for 5 minutes. Protein bands were detected using VersaDoc ${ }^{\mathrm{TM}}$ XR Imaging System and analyzed using Image Lab (BioRad).

\subsubsection{Statistical analyses}

All data were analyzed using GraphPad Prism software (GraphPad Software Inc) and displayed as mean \pm standard error of the mean. $n$ indicates the number of samples whether monolayer or BHM analyzed. Statistical analyses used were t-test, one-way or two-way ANOVA unless stated otherwise. Results showing $\mathrm{P}<0.05$ were considered significant.

For more details about the constituents of the reagents used, please refer to the Appendix. 


\section{Results}

The results of this thesis are divided into three main chapters as indicated in the Introduction.

\subsection{Characterization of a Bioengineered Heart Muscle}

\subsubsection{Directed self-assembly of bioengineered heart muscle}

hPSC can differentiate and self-assemble into BHM in a collagen hydrogel when subjected to sequential addition of growth factors and small molecules as described in 2.3.2. BHM development is characterized by (1) mesoderm induction for 3 days in the presence of a small molecule Wnt signaling activator CHIR99021 (1 $\mu \mathrm{mol} / \mathrm{L})$, FGF-2 (5 ng/mL), Activin-A (9 $\mathrm{ng} / \mathrm{mL})$ and BMP4 (5 ng/mL), (2) cardiac specification for 10 days in the presence of the Wnt signaling inhibitor IWP4 (5 $\mu \mathrm{mol} / \mathrm{L})$ and (3) cardiac maturation from culture day 13 on under mechanical stimulation, TGF $\beta-1(1 \mathrm{ng} / \mathrm{mL})$ and increase in extracellular calcium from 0.4 $\mathrm{mmol} / \mathrm{L}$ to physiological concentrations of $1.2 \mathrm{mmol} / \mathrm{L}$. Cardiac maturation was maintained for up to 50 days in this study. BHM developed under these conditions served as controls unless indicated otherwise. The notable difference from other directed differentiation protocols is the parallel development of cardiomyocytes and stroma cells in a three dimensional growth context from the same starting cell source, namely human pluripotent stem cells.

In the following sections molecular, functional and morphological properties of the BHM model are presented along with proof-of-concept that this model can be used for applications in simulations of cardiac development, disease modeling and drug screening.

\subsubsection{Gene expression profiling of developing bioengineered heart muscle}

BHMs at different time points of development were subjected to RNA extraction. RNA was then subjected to sequencing followed by a bioinformatics analysis of the resulting data. RNA sequencing data revealed that BHM followed the expected developmental patterns of mesoderm 
induction with the reduction of the pluripotency markers such as OCT4 and a transient induction of mesodermal markers (i.e. MESPI and MIXL1) peaking at day 3. Cardiac specification was characterized by the expression of cardiac progenitor markers including NKX2-5 and ISLI (peak at day 8) with concurrent increase in the expression of TBX5. The cardiac maturation phase was characterized by the expression of definitive cardiac markers MYH6, MYH7 and MYL2 (Fig. 16). The increase of the MYH7/MYH6 ratio, although still not at adult heart levels $(28 \pm 5$ for adult heart [kindly provided by Dr. Karl Toicher] vs. $0.4 \pm 0.2$ for BHM at day $22 ; n=4$ /group), may be interpreted as a molecular sign of BHM maturation.
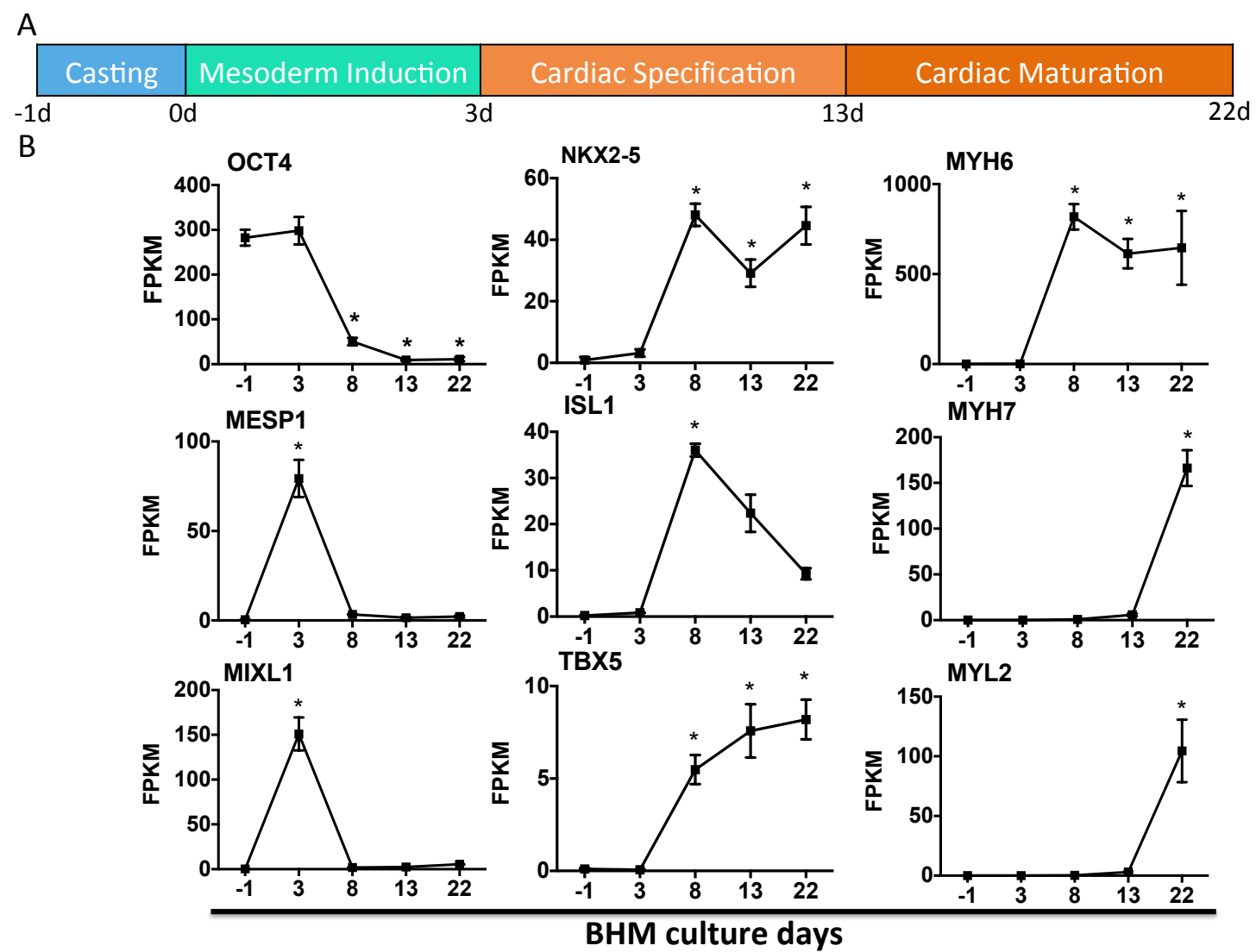

Figure 16: Molecular profiles during BHM development.

(A) Outline of the BHM differentiation protocol as developed by Hudson et al. (in revision). (B) Analysis of RNA sequencing data for pluripotency (OCT4) and early mesoderm (MIXL1, MESP1), cardiac progenitor (NKX2-5, ISL1, TBX5) and cardiomyocyte markers (MYH6, MYH7, MYL2). n=4 BHMs/time point from three independent experiments. $* P<0.05$ vs. $-1 \mathrm{~d}$ by ANOVA with Dunnett's multiple comparison post-hoc test. BHM: bioengineered heart muscle; OCT4: octamer-binding transcription factor 4; MIXL1: mix paired-like homeobox 1; MESP1: mesoderm posterior basic helix-loop-helix transcription factor 1; NKX2-5: NK2 transcription factor related locus 5; ISL1: islet 1; TBX5: T-box transcription factor 5; MYH6: myosin heavy chain 6; MYH7: myosin heavy chain 7; MYL2: myosin light chain 2; d: days; FPKM: fragments per kilobase of exon per million fragments. 


\subsubsection{Cell composition of bioengineered heart muscle}

BHMs were subjected to enzymatic digestion at different time points of differentiation to determine the total cell count. The analyses showed a gradual increase in cell number reaching more than three times of the input cell number by day $13\left(2.3 \pm 0.2 \times 10^{6}\right.$ cells at day 13 vs. 0.5 $\mathrm{x} 10^{6}$ at day $-1 ; n=4 ; \mathrm{p}<0.05$; Fig. $17 \mathrm{~A}$ ), after which this number dropped again markedly to $0.8 \pm 0.1 \times 10^{6}$ cells by culture day 22 (Fig. 17A). Flow cytometry for different markers at culture day 22 revealed that BHMs were composed mainly of $43 \pm 2 \%$ cardiomyocytes marked by $\alpha$ actinin and $29 \pm 2 \%$ stroma cells marked by CD90 with minimal presence of hematopoietic cells (marked by CD117 and CD45) and endothelial cells (marked by CD31) (Fig. 17B). Further analysis of RNA-sequencing data demonstrated transcriptional activity suggesting epicardial (WT1 and ALDH1A2) and endocardial (CD31 and VE-Cadherin) developmental processes starting at the cardiac specification phase and continuing until day 22 (Fig. 17C and E). This was also observed upon staining for the epicardial marker WT1 at both culture days 13 and 22 showing random distribution of WT1 positive cells within the BHM (Fig. 17D). 
A

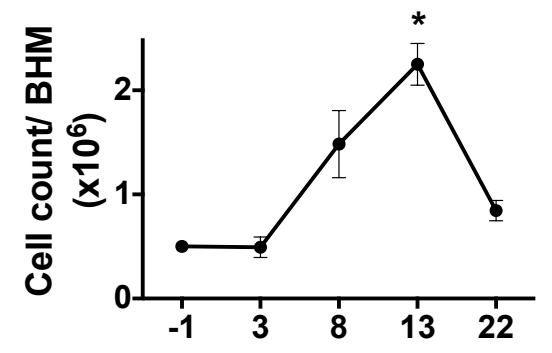

C

Epicardial Development

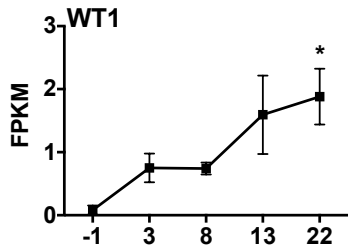

E Endocardial Development
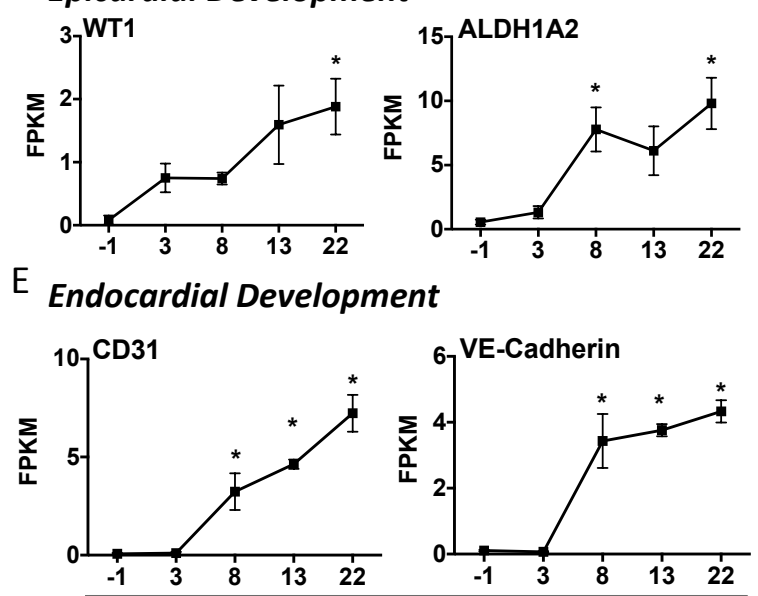

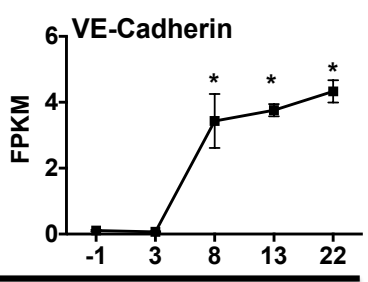

22
B

\begin{tabular}{|c|c|c|}
\hline Marker & Cell type & $\begin{array}{c}\% \text { of total } \\
\text { cells in BHM }\end{array}$ \\
\hline Actinin & Cardiomyocytes & $43 \pm 2 \%$ \\
\hline CD90 & Stroma cells & $29 \pm 2 \%$ \\
\hline CD117 & Hematopoetic cells & $8 \pm 1 \%$ \\
\hline CD45 & Hematopoetic cells & $4 \pm 0.2 \%$ \\
\hline CD31 & Endothelial cells & $2 \pm 0.2 \%$ \\
\hline
\end{tabular}

D

\section{Day 13}
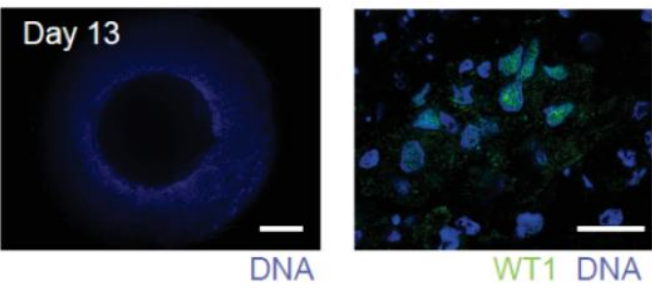

\section{Day 22}

BHM culture days

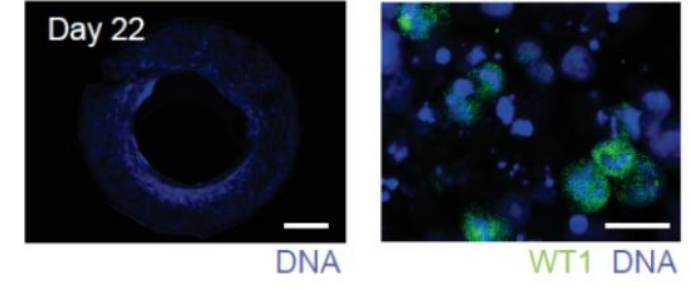

Figure 17: Changes of cell number and composition of BHM.

(A) Total cell count per BHM during differentiation ( $n=4$ BHMs/time point). $* P<0.05$ vs. $-1 \mathrm{~d}$ by ANOVA with Dunnett's multiple comparison post-hoc test. (B) Flow cytometry analysis of the BHM cell composition at culture day 22. (C) RNA sequencing data analyses for canonical epicardial markers (WT1, ALDH1A2); $n=4$ BHMs/time point from three independent experiments. ${ }^{*} P<0.05$ vs. $-1 \mathrm{~d}$ by ANOVA with Dunnett's multiple comparison post-hoc test (D) Whole-mount staining for WT1 (green) and DNA (blue) marking putative epicardial cells in BHMs isolated at day 13 and day 22; Overview of BHM (left panel; scale bar: $2 \mathrm{~mm}$ ) and magnified region (right panel; scale bar: $20 \mu \mathrm{m}$ ) are displayed. (E) RNA sequencing data showing endocardial-like activity (CD31 and $V E$ Cadherin gene expression) in BHMs ( $n=4 \mathrm{BHMs} / \mathrm{time}$ point from three independent experiments). ${ }^{*} P<0.05$ vs. 1d by ANOVA with Dunnett's multiple comparison post-hoc test. BHM: bioengineered heart muscle; CD90: thy-1 cell surface antigen; CD117: kit proto-oncogene receptor tyrosine kinase; CD45: leukocyte common antigen; CD31: platelet/endothelial cell adhesion molecule; WT1: Wilms tumor 1; ALDH1A2: aldehyde dehydrogenase 1 family member A2; VE-Cadherin: vascular endothelin-cadherin; DNA: deoxyribonucleic acid; FPKM: fragments per kilobase of exon per million fragments. 


\subsubsection{Functional properties of bioengineered heart muscle}

\subsubsection{Isometric force measurement}

Spontaneous beating of BHMs was detected by culture day 13 prior to mechanical stretching and continued so until functional analysis at day 22. Isometric force measurements revealed positive inotropic response to increasing extracellular calcium and $\beta$-adrenergic stimulation with isoprenaline $(1 \mu \mathrm{mol} / \mathrm{L})$ as shown in Fig. $18 \mathrm{~A}$ and $\mathrm{B}$, respectively. The apparent half maximal effective concentration $\left(\mathrm{EC}_{50}\right)$ of calcium was calculated to be $0.4 \pm 0.1 \mathrm{mmol} / \mathrm{L}(n=20)$.

A

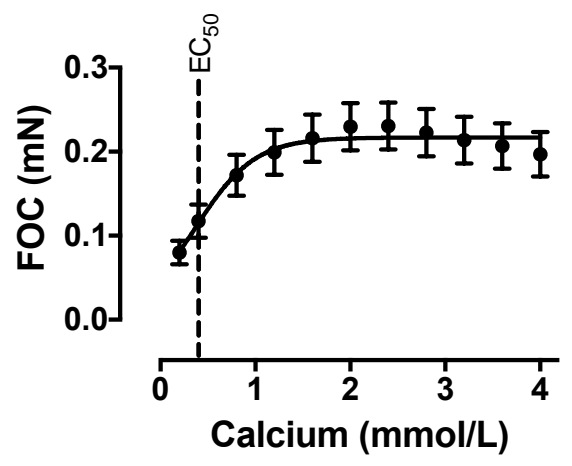

B

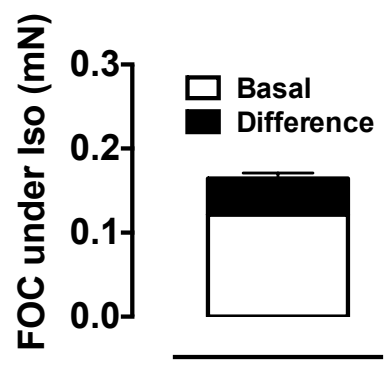

Figure 18: Functional assessment of culture day 22 BHM.

Isometric force measurements showing positive inotropic response of BHMs to (A) increasing extracellular calcium with an $\mathrm{EC}_{50}=0.4 \pm 0.1 \mathrm{mmol} / \mathrm{L}$ (striped line) and $(\mathbf{B}) \beta$-adrenergic stimulation under isoprenaline $(1 \mu \mathrm{mol} / \mathrm{L})$ at 0.6 $\mathrm{mmol} / \mathrm{L}$ calcium is represented in the bar graph $(n=20 \mathrm{BHMs})$. FOC: force of contraction; $\mathrm{EC}_{50}$ : effective concentration at half maximal effect; Iso: isoprenaline.

\subsubsection{Electrophysiological characterization}

In order to see which of the cardiac specific cell types is predominant in the BHM, action potentials were recorded via impaling the cardiomyocytes in the BHM at day 22. Results showed that a majority of the cardiac cells present a ventricular action potential phenotype with however a high resting membrane potential of $-42 \pm 2 \mathrm{mV}$ along with fewer atrial and pacemaker 
cells (Fig. 19). Ventricular and atrial cardiomyocytes were distinguished by their missing spontaneous depolarization in phase 4 of the action potential and their characteristic repolarization kinetics. Pacemaker cells demonstrated a clear spontaneous depolarization during phase 4 of the action potential.

\begin{tabular}{|c|c|c|c|c|}
\hline$-80^{-1}$ & $1 \mathrm{sec}$ & $1 \mathrm{sec}$ & $1 \mathrm{sec}$ & $1 \mathrm{sec}$ \\
\hline Cell type & Ventricular & Atrial & Pacemaker & Intermediate \\
\hline RMP (mV) & $-42 \pm 2$ & {$[-37,-32]$} & & \\
\hline $\mathbf{V}_{\text {threshold }}(\mathrm{mV})$ & & & {$[-25,-19]$} & $-27 \pm 5$ \\
\hline MDP (mV) & & & {$[-41,-30]$} & $-39 \pm 5$ \\
\hline PP (mV) & & & {$[16,11]$} & $14 \pm 1$ \\
\hline $\mathrm{DDR}(\mathrm{mV} / \mathrm{s})$ & & & {$[99,53]$} & $19 \pm 4$ \\
\hline APA $(m V)$ & $64 \pm 2$ & {$[54,44]$} & {$[62,45]$} & $58 \pm 6$ \\
\hline $\mathrm{APD}_{20}(\mathrm{~ms})$ & $82 \pm 5$ & {$[38,63]$} & {$[73,95]$} & $116 \pm 7$ \\
\hline $\mathrm{APD}_{50}(\mathrm{~ms})$ & $112 \pm 6$ & {$[71,89]$} & {$[93,137]$} & $147 \pm 8$ \\
\hline$A P D_{90}(\mathrm{~ms})$ & $138 \pm 8$ & {$[107,113]$} & {$[108,166]$} & $175 \pm 9$ \\
\hline Frequency $(\mathrm{Hz})$ & $1 \pm 0,1$ & {$[1.3,1.0]$} & {$[3.4,1.8]$} & $1.1 \pm 0,2$ \\
\hline $\mathrm{dV} / \mathrm{dt}_{\max }(\mathrm{V} / \mathrm{s})$ & $13 \pm 3$ & {$[16,6]$} & {$[7,6]$} & $8 \pm 1$ \\
\hline$n$ & 17 & 2 & 2 & 6 \\
\hline
\end{tabular}

Figure 19: Electrophysiological properties of cardiomyocytes in BHM at culture day 22.

Action potential recordings demonstrated predominant ventricular development in BHMs with some evidence for the presence of atrial and pacemaker phenotypes ( $n=27$ cells in total from 9 BHMs). RMP: resting membrane potential; $\mathrm{V}_{\text {threshold }}$ : threshold potential; MDP: maximum diastolic potential; PP: pacemaker potential; DDR: diastolic depolarization rate; APA: action potential amplitude; APD: action potential duration; n: number of cells demonstrating respective action potential phenotype.

\subsubsection{Cardiac ion channel and calcium homeostasis associated gene expression}

RNA-sequencing data revealed the expression of not only most common cardiac ion channels (SCN5A, HCN4, CACNA1C, and KCNH2), but also of canonical cardiac calcium homeostasis associated genes (RYR2, ATP2A2, PLN and CASQ2) in BHMs at day 22 (Fig. 20). 
A
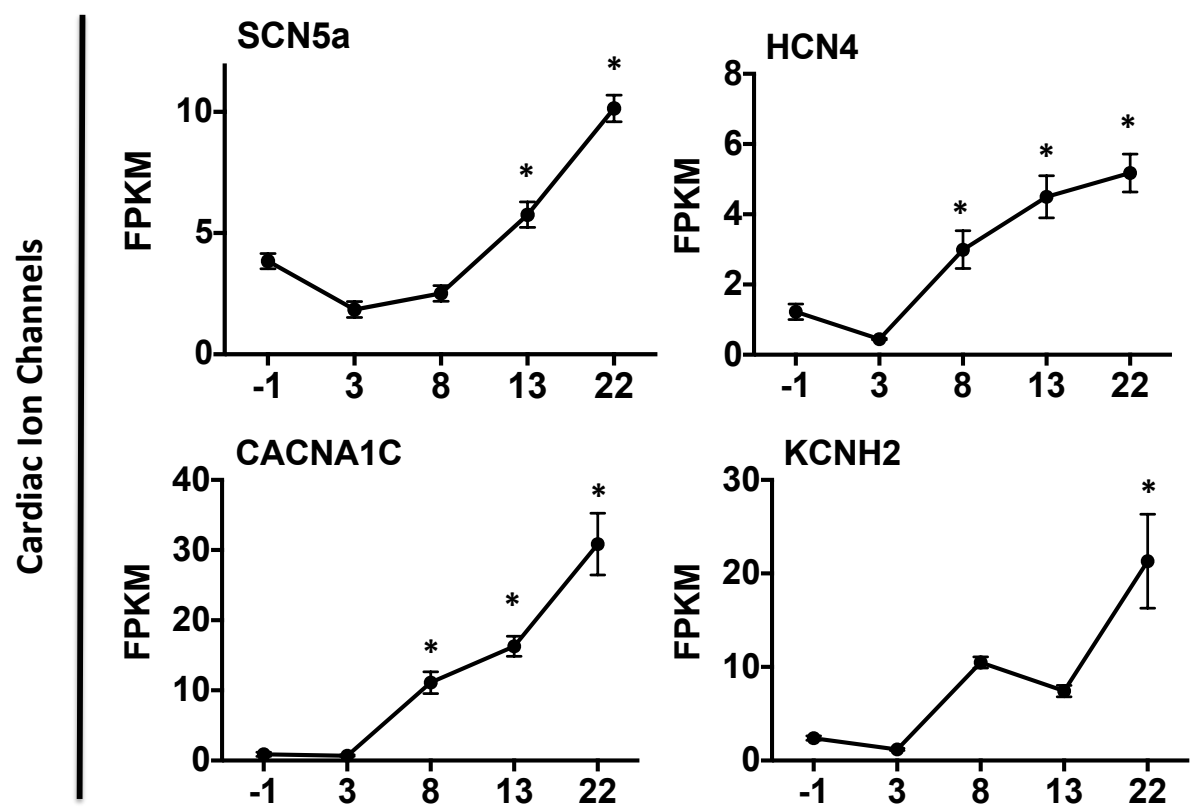

B

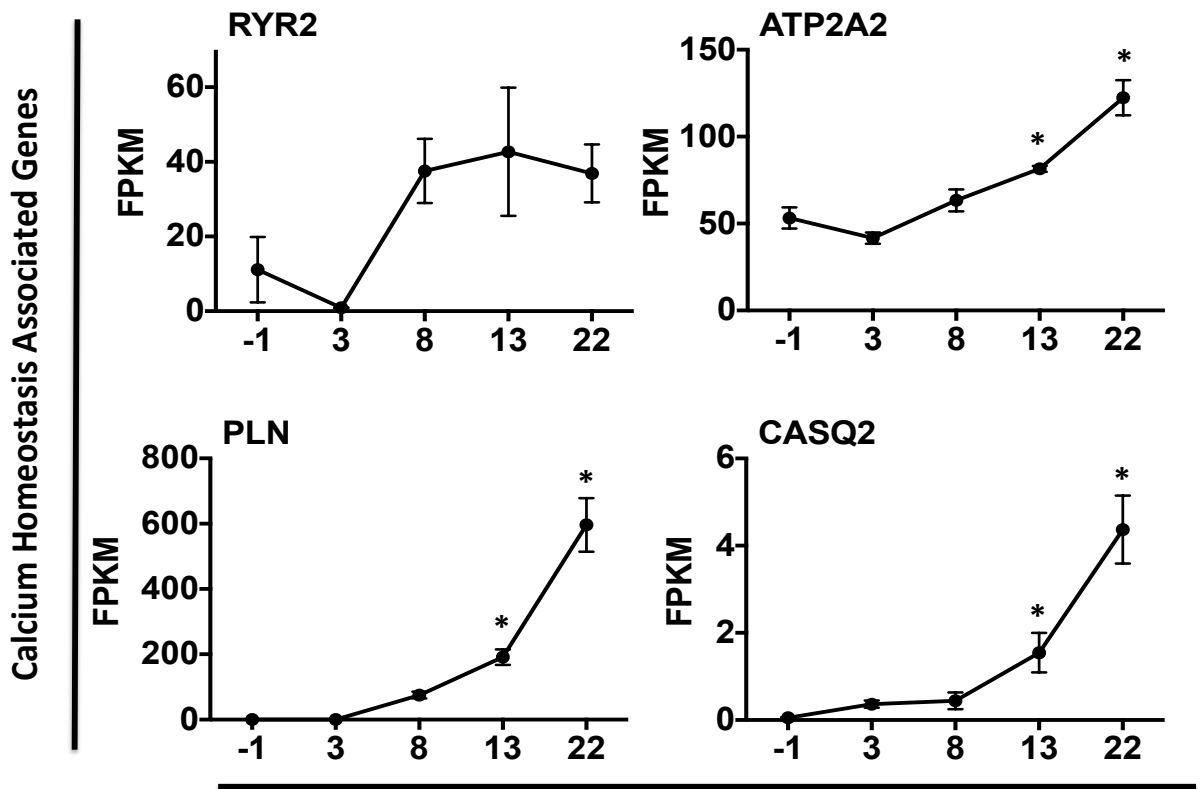

BHM culture days

Figure 20: Expression profile for cardiac ion channels and calcium homeostasis associated genes.

RNA-sequencing data analyses showed an increased abundance of transcripts encoding (A) cardiac ion channels (SCN5a, HCN4, CACNAIC, KCNH2) and (B) calcium homeostasis associated genes (RYR2, ATP2A2, PLN, $C A S Q 2)$ in BHM ( $n=4 \mathrm{BHMs} /$ time point from three independent experiments). $* P<0.05$ vs. $-1 \mathrm{~d}$ by ANOVA with Dunnett's multiple comparison post-hoc test. SCN5a: sodium voltage-gated channel alpha subunit 5; HCN4: hyperpolarization activated cyclic nucleotide gated-potassium channel 4; CACNA1C: calcium voltage-gated channel subunit alpha1 C; KCNH2: potassium voltage gated-channel subfamily H member 2; RYR2: ryanodine receptor 2; ATP2A2: ATPase sarcoplasmic/endoplasmic reticulum calcium transporting 2; PLN: phospholamban; CASQ2: calsequestrin; BHM: bioengineered heart muscle; FPKM: fragments per kilobase of exon per million fragments. 


\subsubsection{Continuous maturation of bioengineered heart muscle by day 60}

To investigate whether maturation in BHM is progressing with time, BHM from culture days 29 and 60 were subjected to functional, morphological and molecular analyses and compared to day 22 BHM cultures. These extended cultures demonstrated the following functional properties: (1) cellular calcium (maximal FOC: $0.31 \pm 0.18 \mathrm{mN}$ at day 60 vs. $0.21 \pm 0.07 \mathrm{mN}$ at day $22 ; n=20 ; \mathrm{p}<0.05$ ) and $\beta$-adrenergic stimulation (increase in FOC: $65 \pm 4 \%$ at day 60 vs. $37 \pm 3 \%$ at day $22 ; n=20)$ as well as a right-shift of the calcium response curve $\left(\mathrm{EC}_{50} \mathrm{in} \mathrm{mmol} / \mathrm{L}\right.$ : $0.6 \pm 0.2$ at day 60 vs. $0.4 \pm 0.1$ at day $22 ; n=20$; Fig. $21 \mathrm{~A}$ and B); (2) decrease in spontaneous beating rate from $80 \pm 3 \mathrm{bpm}$ at day 22 to $65 \pm 3 \mathrm{bpm}$ at day 60 ( $n=20$ respectively; Fig. $21 \mathrm{C}$ ); (3) a significant drop not only in cross sectional area (CSA: $1 \pm 0.2 \mathrm{~mm}^{2}$ at day 60 vs. $1.9 \pm 0.2 \mathrm{~mm}^{2}$ at day 22; $n=8-10 ; \mathrm{p}<0.05 ;$ Fig. 21D), but also in total BHM cell count and specifically the cardiomyocyte content $(18 \pm 2 \% ; n=15-20 ; \mathrm{p}<0.05$ vs. day 22 ; Fig. $21 \mathrm{E})$. 
A

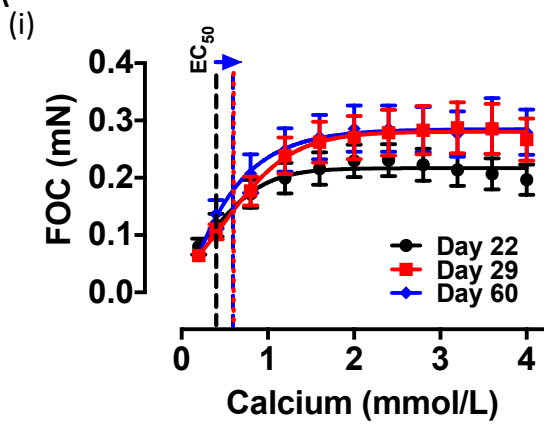

(ii)

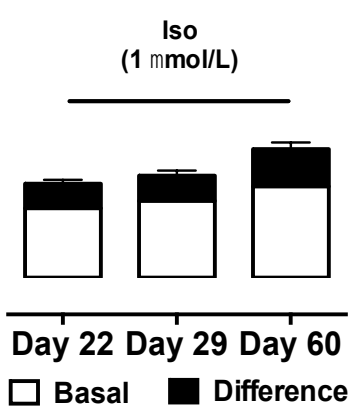

B

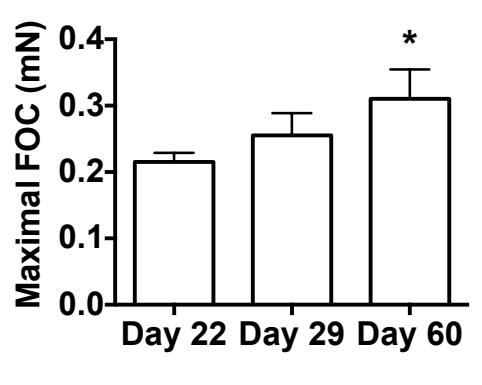

E

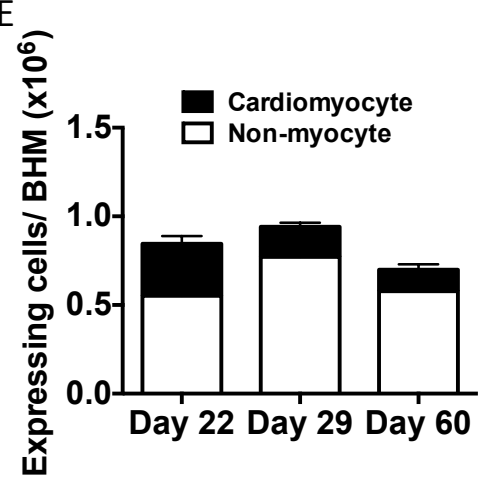

Figure 21: Functional, morphological and cell count analyses of BHMs in long-term cultures.

(A) (i) Force of contraction (FOC) in response to increasing extracellular calcium concentrations measured under isometric conditions after 22, 29 and 60 days of culture; calcium concentrations with half maximal effect $\left(\mathrm{EC}_{50}\right)$ are indicated with dotted lines $(n=20 \mathrm{BHMs} /$ time point). (ii) Inotropic response to $\beta$-adrenergic stimulation with isoprenaline $(1 \mu \mathrm{mol} / \mathrm{L})$ at $0.6 \mathrm{mmol} / \mathrm{L}$ calcium is represented in the bar graph $(n=20 \mathrm{BHMs} /$ time point $)$. (B) Maximal FOC generated by BHMs after 22, 29, and 60 days of culture ( $n=20 \mathrm{BHMs} /$ time point). $* P<0.05$ vs. Day 22 by ANOVA with Dunnett's multiple comparison post-hoc test $(\mathbf{C})$ Beating rate recorded for BHMs after 22, 29, and 60 days of culture ( $n=20 \mathrm{BHMs} /$ time point). (D) Analysis of BHM cross sectional area after 22, 29, and 60 days of culture ( $n=8 \mathrm{BHMs} /$ time point). $* P<0.05$ by ANOVA with Tukey's multiple comparison post-hoc test (E) Cardiomyocyte ( $\alpha$-actinin) and non-myocyte (CD90) content in BHMs after 22, 29, and 60 days of culture ( $n=15-20 \mathrm{BHMs} /$ time point). FOC: force of contraction; CSA: cross sectional area; Iso: isoprenaline.

The drop in cardiomyocyte content and cross sectional area along with the increase in maximal force of contraction generated by the BHMs at day 60 suggested a markedly enhanced contractile performance of individual cardiomyocytes $(4.6 \pm 1.3 \mathrm{nN} /$ cardiomyocyte at day 60 vs. $1.1 \pm 0.5 \mathrm{nN} /$ cardiomyocyte at day $22 ; n=20 ; \mathrm{p}<0.001 ;$ Fig. $22 \mathrm{~A})$ on the cellular level as well as per muscle bundle area on the tissue level $\left(0.35 \pm 0.28 \mathrm{mN} / \mathrm{mm}^{2}\right.$ at day 60 vs. $0.13 \pm 0.10$ $\mathrm{mN} / \mathrm{mm}^{2}$ at day $22 ; n=8-11 ; \mathrm{p}<0.05$; Fig. $\left.22 \mathrm{~B}\right)$. At the ultrastructural level, no obvious differences of sarcomere assembly could be observed; at all time points well developed Z-, Iand A- bands were present (Fig. 22C). On the gross structural level, it appeared that day 60 BHM contained better aligned muscle networks in comparison to a 22 day-old BHM (Fig. 22D). 
A

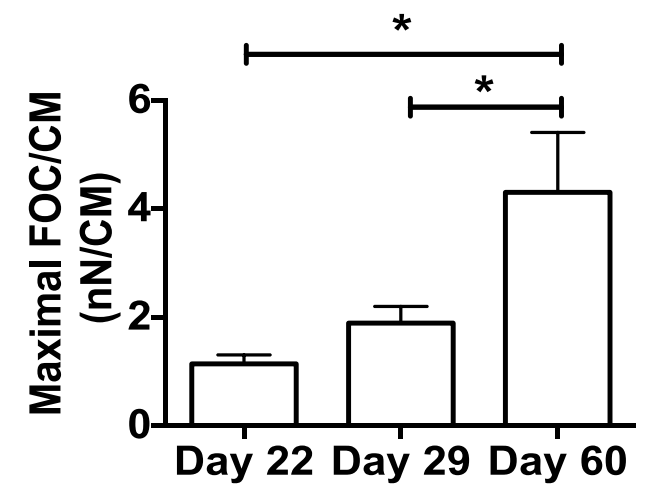

B

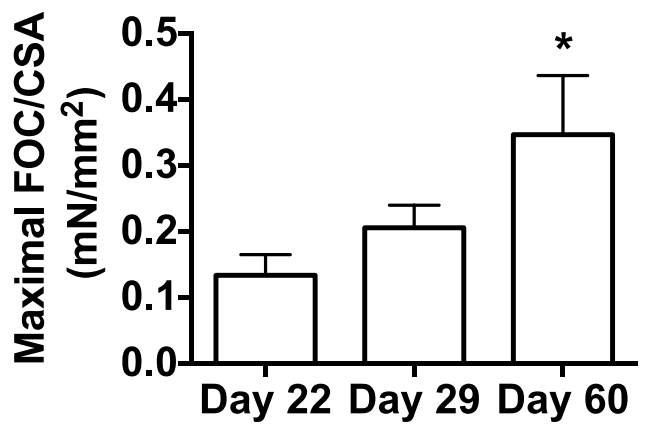

C

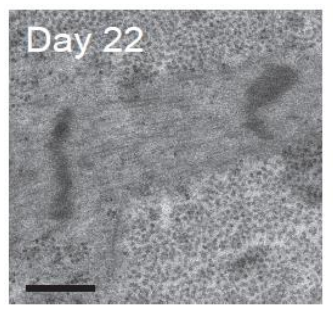

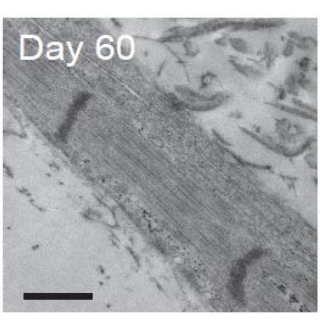

D
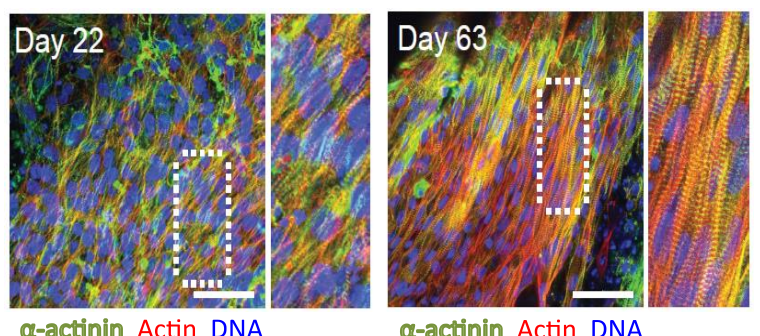

a-actinin Actin DNA

Figure 22: Further functional and morphological maturation in long-term BHM cultures.

(A) Maximal FOC per individual BHM cardiomyocyte $(n=20 \mathrm{BHMs} /$ time point). $* P<0.05$ by ANOVA with Tukey's multiple comparison post-hoc test. (B) Maximal FOC per cross sectional area (CSA; $n=8-11$ BHMs/time point) after 22, 29 and 60 days of culture. $* P<0.05$ vs. Day 22 by ANOVA with Dunnett's multiple comparison post-hoc test. (C) Ultrastructural analysis of sarcomeres in 22 and 60 day-old BHMs by transmission electron microscopy; scale bar: $500 \mathrm{~nm}$. (D) Whole mount immunostaining of BHMs on day 22 and 63 ; $\alpha$-actinin (green), actin (red) and DNA (blue); scale bar: $50 \mu \mathrm{m}$. Confocal images were recorded by James E. Hudson. FOC: force of contraction; CM: cardiomyocyte.

Next, RNA-sequencing was performed on BHMs on day 29 and day 60 to investigate the transcriptional activity of cardiac genes possibly involved in the enhanced force of contraction. This analysis is challenging because of the differences in cardiomyocyte content in BHM at culture days 22, 29 and 60. Thus, we investigated the RNAseq data sets and found that ACTC1 (cardiac actin) correlated closely with cardiomyocyte content (Fig. 23). For this reason, we used ACTC1 as a cardiomyocyte housekeeping gene to correct for differences in cardiomyocyte content in the following analysis of cardiomyocyte specific transcripts in BHM from culture days 22, 29 and 60 (Fig. 24). 


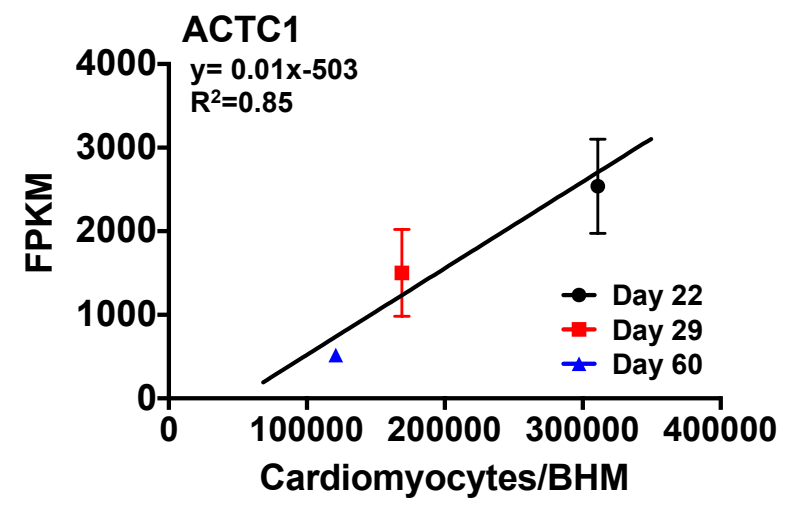

Figure 23: Correlation of cardiomyocyte content and cardiac actin (ACTC1) in BHM.

ACTC1 transcript abundance assessed by RNAseq is displayed on the ordinate. Cardiomyocyte content in BHM assessed after enzymatic dispersion on culture days 22, 29 and 60 is displayed on the abscissa. A correlation analysis by linear regression was performed ( $n=4 \mathrm{BHMs} /$ time point). ACTC1: actin alpha cardiac muscle 1; BHM: bioengineered heart muscle; FPKM: fragments per kilobase of exon per million fragments.

This analysis did indeed suggest, in agreement with the functional and morphological data, that further molecular maturation of cardiomyocytes in BHM occurred between culture days 22 and 29 with stable levels of expression until day 60; evidenced by enhanced expression of TNNI3 and MYH7 (Fig. 24). Expression of the latter gene was markedly enhanced while MYH6 decreased, resulting in a further increase in $M Y H 7 / M Y H 6$ transcript ratio (from $0.3 \pm 0.2$ at day 22 to $1.1 \pm 0.04$ at day $60 ; n=4 ; \mathrm{p}<0.05 ;$ Fig. 24$)$ towards ratios observed in the adult heart $(28 \pm 5$; [kindly provided by Dr. Karl Toicher]) 


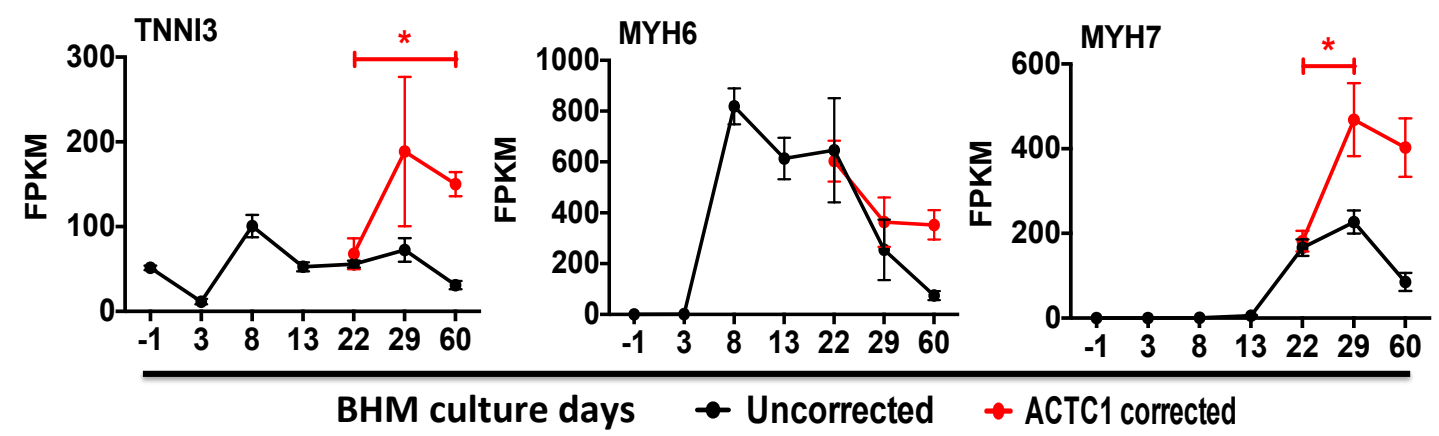

Figure 24: Continuous molecular maturation in BHM with culture.

Individual trajectory of genes transcribed in cardiomyocytes (TNNI3, MYH6 and MYH7). Correction of cardiomyocyte abundance was done by correlation of transcripts to ACTC1 as cardiomyocyte housekeeping gene for the data from BHM culture days 22-60 (red curve); uncorrected transcript abundance is depicted for comparison (black curve; $n=4 \mathrm{BHMs} /$ time point from three independent experiments). $* P<0.05$ vs. $22 \mathrm{~d}$ by ANOVA with Tukey's multiple comparison post-hoc test. TNNI3: troponin I3 cardiac type; MYH6: myosin heavy chain 6; MYH7: myosin heavy chain 7; FPKM: fragments per kilobase of exon per million fragments.

Similar analyses of transcripts encoding for proteins involved in cardiac muscle contraction (SCN5a, KCNH2 and HCN4; Fig. 25A) and calcium homeostasis (RYR2, CASQ2, and ATP2A2;

Fig. 25B) confirmed the findings above and suggest that BHM maturation is a continuous process in BHM culture. 
A

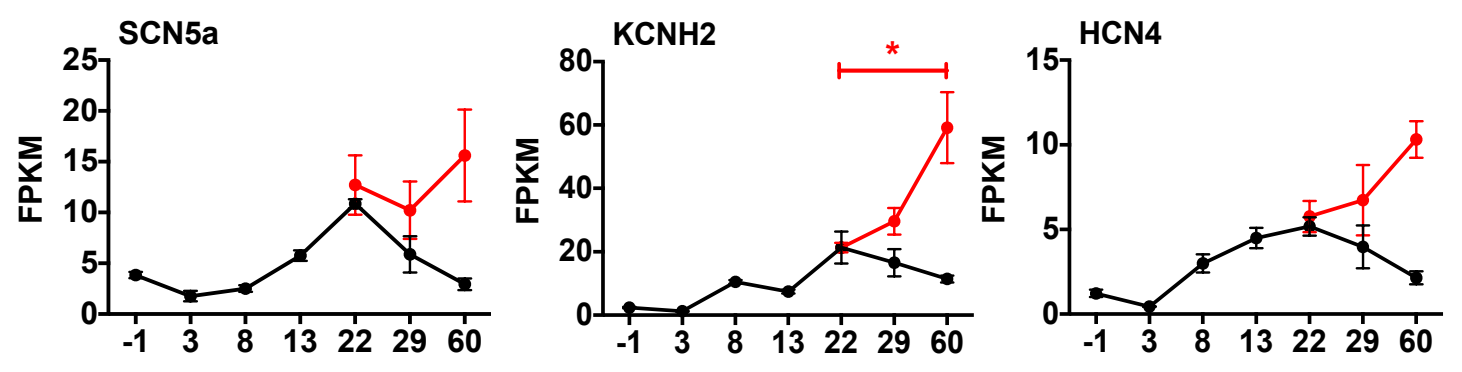

B
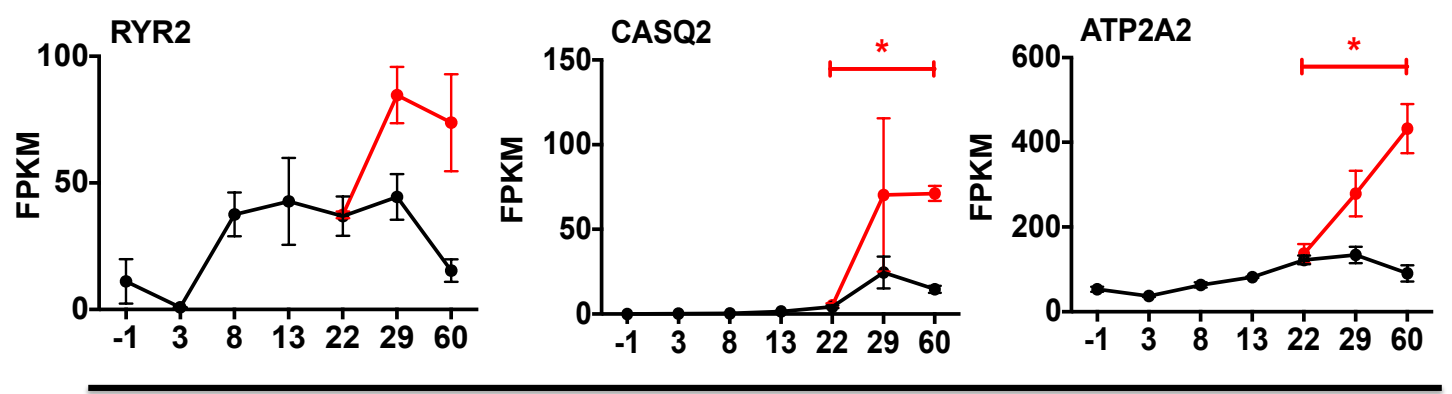

BHM culture days

- Uncorrected

ACTC1 corrected

Figure 25: Enhanced cardiac ion channel and calcium homeostasis associated gene expression.

Individual trajectories of genes transcribed in cardiomyocytes involved in (A) cardiac ion channel for function (SCN5a, KCNH2 and HCN4) and (B) calcium homeostasis (RYR2, CASQ2, ATP2A2). Correction cardiomyocyte abundance was done by correlation of transcripts to ACTCl as cardiomyocyte housekeeping gene for the data from BHM culture days 22-60 (red curve); uncorrected transcript abundance is depicted for comparison (black curve) ( $n=4 \mathrm{BHMs} /$ time point from three independent experiments). ${ }^{*} P<0.05$ vs. $22 \mathrm{~d}$ by ANOVA with Dunnett's multiple comparison post-hoc test. SCN5a: sodium voltage-gated channel alpha subunit 5; KCNH2: potassium voltage gated-channel subfamily $\mathrm{H}$ member 2; HCN4: hyperpolarization activated cyclic nucleotide gatedpotassium channel 4; RYR2: ryanodine receptor 2; CASQ2: calsequestrin; ATP2A2: ATPase sarcoplasmic/endoplasmic reticulum calcium transporting 2; ACTC1: actin alpha cardiac muscle 1; BHM: bioengineered heart muscle; FPKM: fragments per kilobase of exon per million fragments. 


\subsubsection{Evidence for cardiac-neuro crosstalk during BHM development}

In an effort to determine cell composition and associated biological processes in BHM, we evaluated our RNAseq data by an unbiased bioinformatic algorithm (SOTA; self-organizing tree algorithm; Hudson et al., in revision). This analysis identified 4 major processes, according to Kyoto Encyclopedia of Genes and Genomes (KEGG) pathways, associated with BHM development: (1) cardiomyogenesis, (2) loss of potency, (3) neural crest development and (4) metabolism (Fig. 26).
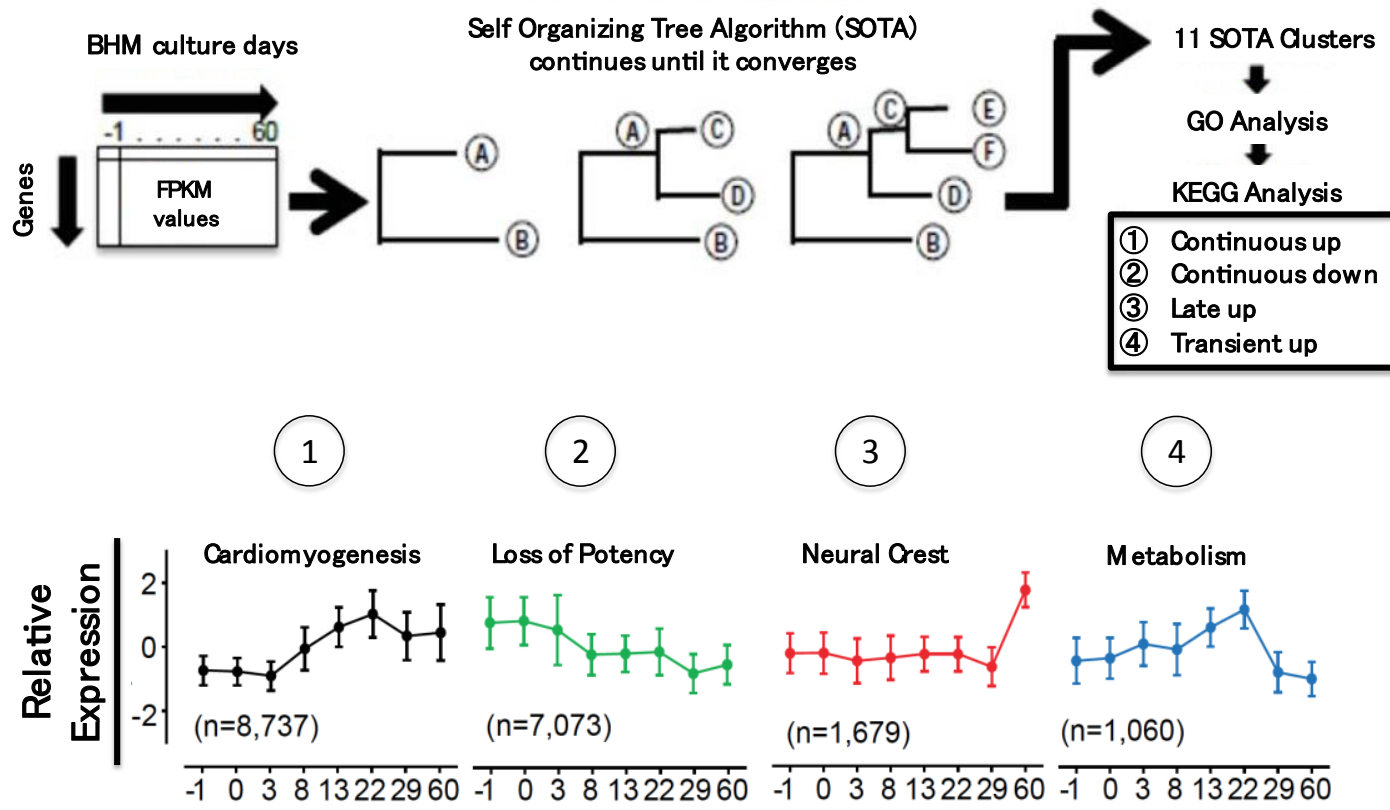

BHM culture days

Figure 26: SOTA analysis of BHM transcriptomes.

Bioinformatics analyses using self-organizing tree algorithm (SOTA) identified clusters with similar gene ontologies according to 4 biological processes: (1) cardiomyogenesis, (2) loss of potency, (3) neural crest and (4) metabolism. BHM: bioengineered heart muscle; FPKM: fragments per kilobase of exon per million fragments; SOTA: self-organizing tree algorithm; GO: gene ontology; KEGG: kyoto encyclopedia of genes and genomes; $\mathrm{n}$ : number of transcripts assigned specifically to individual clusters.

The evidence of neural crest development in late BHM cultures was particularly interesting and prompted us to pay attention to the transcription of factors involved in neural development such 
as NEUROD1, PAX6 and TH (Fig. 27A); all were upregulated and in particular the upregulation of $T H$ as a pivotal enzyme in catecholamine synthesis in the sympathetic system supported the notion that neural crest developed. This was subsequently confirmed by stainings for TH in culture day 60 BHM (Fig. 27B); note that we did not observe any morphological evidence of ectodermal induction at earlier time points of BHM culture. The slightly enhanced PAX6 transcription after day 8 in BHM development with concurrent increases in the $T H$ and NEUROD1 (Fig. 27A arrow) did however suggest early, and so far unnoted, ectodermal activity in parallel to the functional maturation of BHM. More detailed morphological studies identified neuro-spheres, especially at the outer surface of BHM in close proximity to networks of wellorganized striated cardiomyocyte bundles (Fig. 27C). 

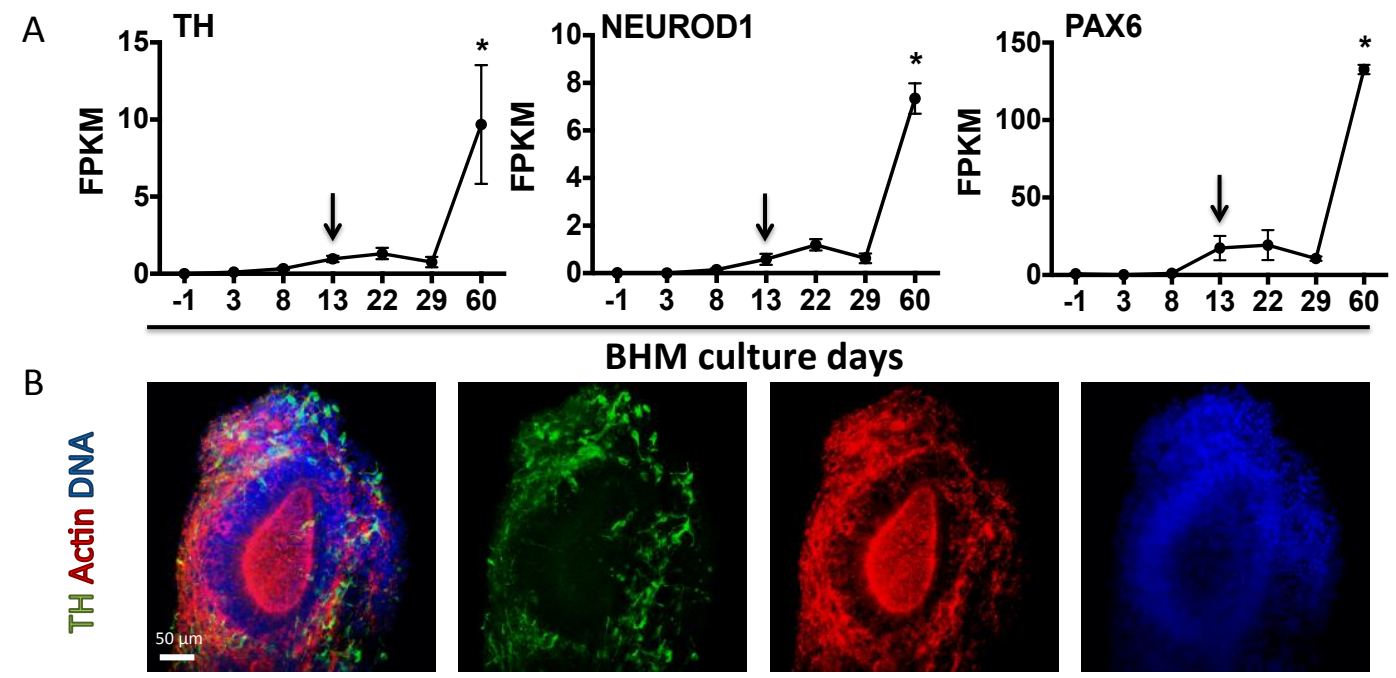

C

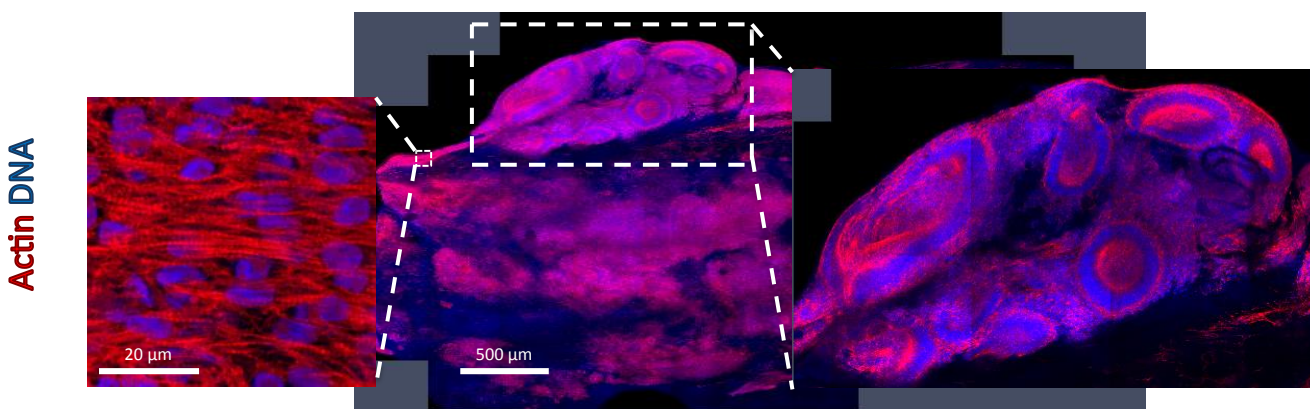

Figure 27: Molecular and morphological evidence for neural crest development in BHM.

(A) Gene expression profiling identified canonical markers of ectoderm and neural crest development as precursors for the sympathetic nerve system (TH, NEURODI and PAX6) with a first sign of expression after culture day 8 and markedly enhanced expression on culture day 60 ( $n=4 \mathrm{BHMs} /$ time point from three independent experiments). * $P$ $<0.05$ vs. $-1 \mathrm{~d}$ by ANOVA with Dunnett's multiple comparison post-hoc test. Immunofluorescence staining of (B) a neurosphere in a day 60 BHM (TH: green, actin: (red), DNA: blue). Scale bar: $50 \mu \mathrm{m}$. (C) Overview of surfacebound neurospheres in close proximity to well-organized cardiomyocyte bundles (actin: red, DNA: blue) in culture day 60 BHM. Scale bar: $500 \mu \mathrm{m}$. Boxes with striped lines indicate magnified regions. Scale bar: $20 \mu \mathrm{m}$. TH: tyrosine hydroxylase; NEUROD1: neuronal differentiation 1; PAX6: paired box 6; BHM: bioengineered heart muscle; DNA: deoxyribonucleic acid; FPKM: fragments per kilobase of exon per million fragments.

In an attempt to investigate whether the identified neural crest-like structures could contribute functionally to BHM development, we sought to block the vesicular monoamine transporter (VMAT2) with reserpine to (1) prevent catecholamine reuptake and thus (2) enhance catecholamine release from functional sympathetic neurons; note that VMAT2 showed a similar expression profiles as the other neuronal markers (Fig. 28B). BHMs were first treated with reserpine for two weeks from culture day 46 to culture day 60 to deplete catecholamine stores and investigate whether its depletion would have a detrimental effect on BHM development. 
Isometric force measurements demonstrated no differences in BHM contractility and thus did not confirm our hypothesis ( $n=6 \mathrm{BHMs} / g r o u p ; ~ F i g . ~ 28 C)$. However upon acute addition of reserpine to the tissues suspended in the organ baths, a progressive drop in beating rate was observed only in the chronically pretreated BHMs (red trace, $n=3$ BHMs/group; Fig. 28D) as opposed to the control tissues, which showed an increase in beating frequency (blue trace, $n=3$ BHMs/group; Fig. 28D). Beating rate was stable in BHM that were niether chronically nor acutely treated with reserpine (black trace; $n=3$ BHMs/group; Fig. 28D). The increase in beating frequency in the not catecholamine depleted BHM suggests off-target effects or catecholamine leak. Conversely, the decrease in beating frequency in the catecholamine depleted BHM suggest a functional sympathetic nerve/neural crest component with a depletion of catecholamine stores in day 60 BHM. Further studies are needed to clarify the neural crest contribution and whether chronic reserpine treatment resulted in "maturation" of the sympathetic nervous component in BHM. 
A

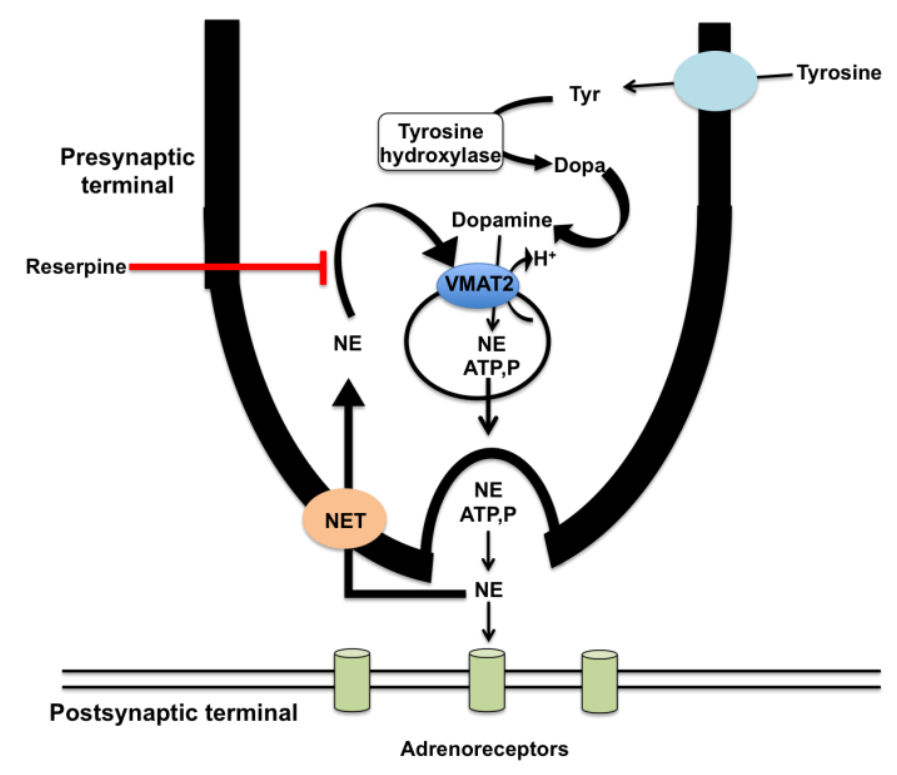

B

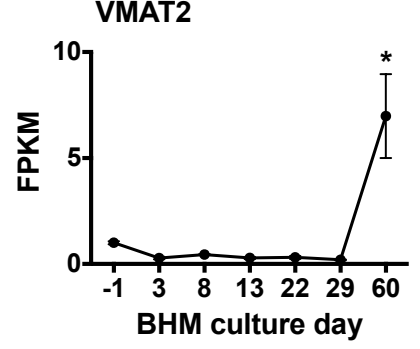

C
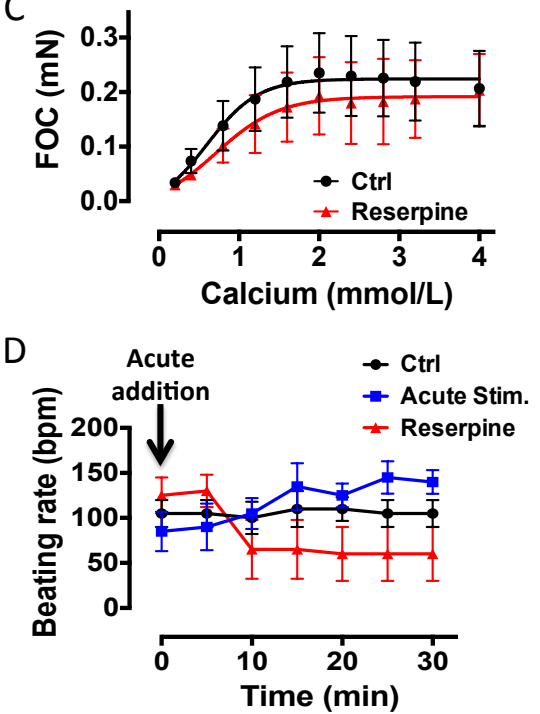

Figure 28: Evidence for a sympathetic component in day $60 \mathrm{BHM}$.

(A) Schematic overview of the action of reserpine by blocking VMAT2 and inhibiting vesicular reuptake at the presynaptic terminal. Image adapted from www.studyblue.com. (B) Transcriptome profiling identified the expression of the vesicular monoamine transporter (VMAT2) in day 60 BHM. $* P<0.05$ vs. $-1 \mathrm{~d}$ by ANOVA with Dunnett's multiple comparison post-hoc test. (C) Depletion of catecholamine storage vesicles by 2 week reserpine $(10 \mathrm{nmol} / \mathrm{L})$ did not result in obvious differences in contractile function of BHM on culture day 60 ( $n=6$ BHMs/group). (D) Acute depletion of catecholamine stores while isometrically suspended in Tyrode's solution at $37^{\circ} \mathrm{C}$ resulted in a decreased spontaneous beating frequency in reserpine pretreated BHM (red line) and an increase in beating frequency in untreated BHM (blue line); controls are in black $(0.001 \%$ DMSO treated). $(n=3$ BHMs/group). Tyr: tyrosine; VMAT2: vesicular monoamine transporter 2; NE: norepinephrin; ATP: adenosine triphosphate; NET: norepinephrin transporter; BHM: bioengineered heart muscle; FOC: force of contraction; FPKM: fragments per kilobase of exon per million fragments; Ctrl: control; Acute Stim: acute stimulation by reserpine. 


\subsubsection{Applications of the BHM model in disease modeling and drug screening}

\subsubsection{Disease modeling}

Studies from mice and chick models have demonstrated the importance of BMP signaling in cardiac cushion formation and myocardial differentiation of cardiac progenitors (Kruithof et al., 2012; Wang et al., 2010). To test whether BMP inhibition would indeed cause a cardiomyogenesis defect in the absence of hemodynamic stresses, we added dorsomorphin (2 $\mu \mathrm{mol} / \mathrm{L}$ ) and a more selective BMP-receptor (ALK2) inhibitor dorsomorphin homologue 1 (DMH1, $2 \mu \mathrm{mol} / \mathrm{L}$ ) during cardiac specification phase. The treatment started on culture day 6 when early cardiac markers were already detected and thus fundamental processes of mesoderm induction were not influenced. The treatment continued until day 22 of BHM culture with subsequent subjection of the BHMs to functional, cellular and structural analyses (Fig. 29A). 
A

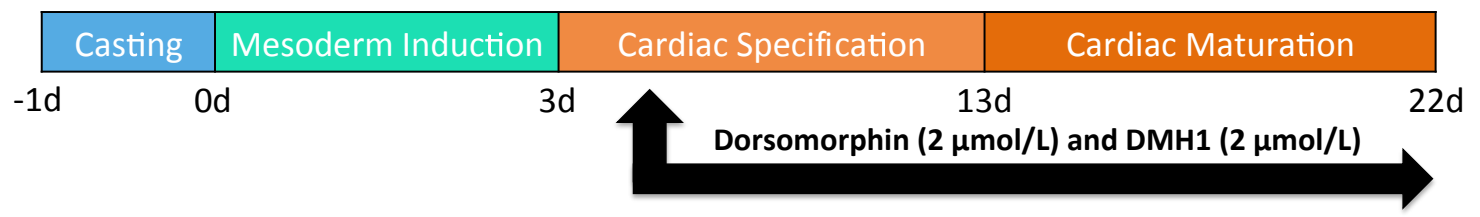

B

C

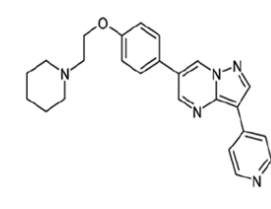

(i)

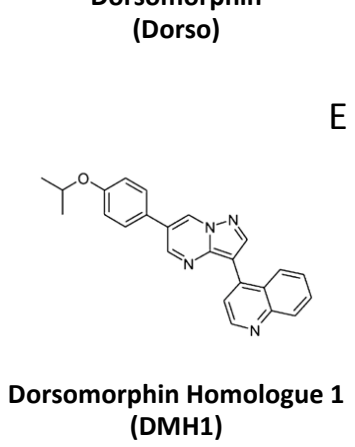

$E$
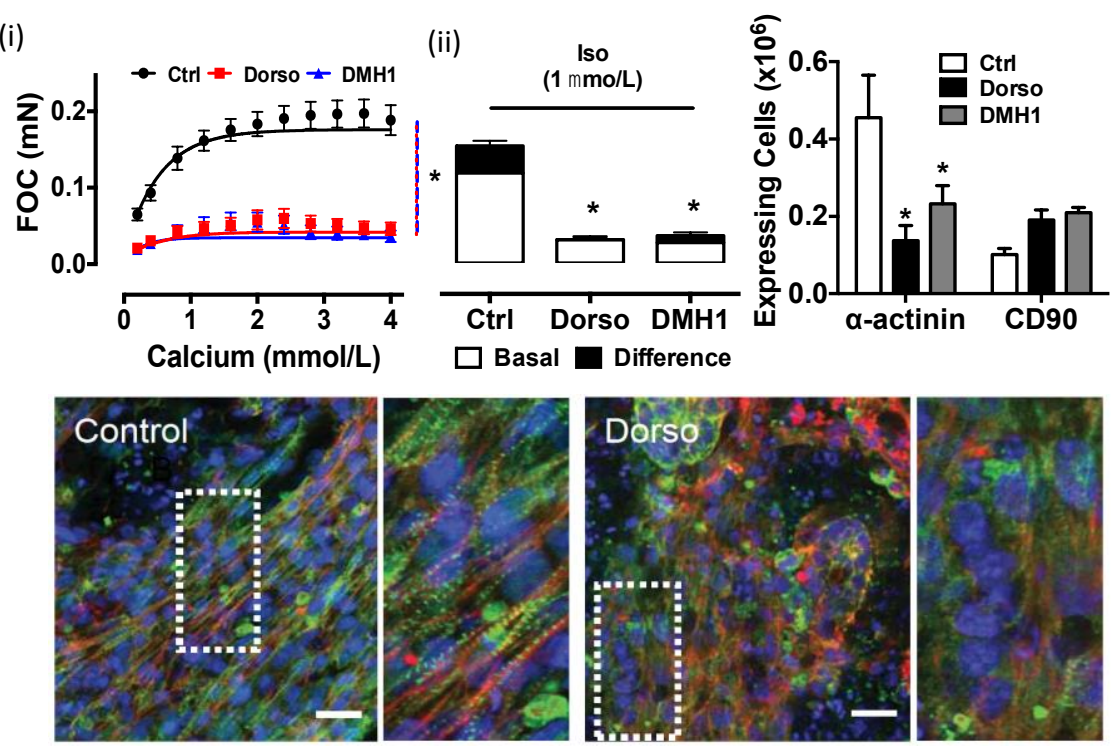

a-Actinin Actin DNA

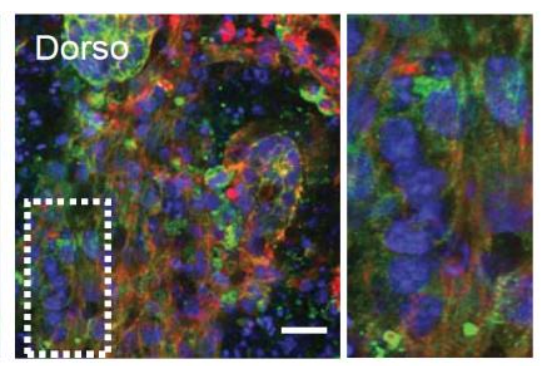

a-Actinin Actin DNA

Figure 29: Identification of faulty cardiomyogenesis in BHM under BMP inhibition.

(A) Outline of the protocol with BMP-signaling blockade using dorsomorphin $(2 \mu \mathrm{mol} / \mathrm{L})$ or DMH1 $(2 \mu \mathrm{mol} / \mathrm{L})$ during culture days 6 to 22 of BHM development. Blockers were applied at concentrations established previously to be effective in biological models (Hao et al. [2008]; Hao et al. [2010]). (B) Chemical structures of Dorso (top) and DMH1 (bottom). (C) FOC examined under (i) increasing extracellular calcium concentrations measured on culture day 22 ( $n=8-12$ BHMs/group). $* P<0.05$ vs. Ctrl by two-way ANOVA with Bonferroni's multiple comparison post-hoc test. (ii) Inotropic response to $\beta$-adrenergic stimulation with isoprenaline $(1 \mu \mathrm{mol} / \mathrm{L})$ at $0.6 \mathrm{mmol} / \mathrm{L}$ calcium is represented in the bar graph. $* P<0.05$ vs. Ctrl by two-way ANOVA with Dunnett's multiple comparison post-hoc test. (D) Flow cytometry for cardiomyocyte ( $\alpha$-actinin) and stromal cell (CD90) content on culture day 22 ( $n=8 \mathrm{BHMs}$ /group). $* P<0.05$ vs. Ctrl by one-way ANOVA with Dunnett's multiple comparison post-hoc test. (E) Immunofluorescence staining of cardiac muscle structures $(\alpha$-actinin: green, actin: red, DNA: blue) of Dorso-treated BHMs vs. Control at culture day 22. Boxes with striped lines indicate magnified regions. Scale bar: $20 \mu \mathrm{m}$. Confocal Image is taken by James E. Hudson. Ctrl: control; Dorso: dorsomorphin; DMH1: dorsomorphin homologue 1; FOC: force of contraction; Iso: isoprenaline; CD90: Thy-1 cell surface antigen; d: days.

On the functional level, at BHM culture day 22 both dorsomorphin and DMH1 treated tissues showed contractile dysfunction with a marked drop in force development (Maximal FOC in $\mathrm{mN}$ : $0.05 \pm 0.03$ and $0.06 \pm 0.03$ respectively vs. $0.2 \pm 0.04$ for Ctrl; $n=7-12 ; \mathrm{p}<0.0001$ vs. Ctrl; Fig. 29C(i)) and inotropic response to isoprenaline stimulation (increase in FOC: $8 \pm 3 \%$ for dorsotreated BHMs and $15 \pm 3 \%$ for DMH1-treated samples vs. $38 \pm 3 \%$ for Ctrl; $n=7-12 ; \mathrm{p}<0.0001$; 
Fig. 29C(ii)). Analyses of the cellular components showed a slight increase in the CD90 stromal cell population upon BMP inhibition as opposed to a significant decrease in cardiomyocyte number $\left(\alpha\right.$-actinin ${ }^{+}$cells: $0.14 \pm 0.05 \times 10^{6}$ and $0.23 \pm 0.07 \times 10^{6}$ upon treatment with Dorso or DMH1 respectively vs. $0.45 \pm 0.13 \times 10^{6}$ for Ctrl; $n=7-12$; p $<0.001$; Fig. 29D). Additionally, whole mount staining of BHM structures revealed impaired cardiac muscle formation upon BMP inhibition in line with the functional and cellular assessments (Fig. 29E).

\subsubsection{Drug screening}

Based on RNA sequencing data which revealed an increase in expression of many important cardiac ion channels by day 29 and 60, the next step was to test the functionality of these channels and consequently test whether BHM can be applied to identify the biological activity of classical ion channel blockers. Accordingly, BHMs at day 29 were treated with increasing concentrations (from $10^{-5}$ to $10 \mu \mathrm{mol} / \mathrm{L}$ ) of (1) digitoxin ( $\mathrm{Na} / \mathrm{K}$-ATPase inhibitor), (2) verapamil (L-Type Calcium channel blocker) and (3) E4031 (HERG blocker) and measured under isometric conditions in the presence/absence of electric field stimulation $(3 \mathrm{~Hz})$. BHM on culture day 29 were chosen due to the increase in overall tissue performance (i.e.: $\mathrm{FOC}, \mathrm{EC}_{50}$ shift in calcium response curve, elevated ion channel expression) compared to 22 day cultured BHM.

Analyses of the inotropic (FOC; Fig. 30A), lusitropic (time-to-50\% relaxation; Fig. 30B) and chronotropic (beating rate; Fig 30C) responses showed prototypic effects of the drugs; treatment with digitoxin lead to an increase in force development, time-to-50\% relaxation and beating rate. Blocking the L-type calcium channel with verapamil resulted in the anticipated negative inotropic response. HERG $\left(\mathrm{I}_{\mathrm{Kr}}\right)$ blockade with reference compound $\mathrm{E} 4031$ prolonged relaxation (Fig 30B) which resulted in a decreased beating rate (Fig. 30C) with no effect on force development (Fig. 30A). The latter observation was in line with action potential recordings of BHMs treated with E4031 (0.5 and $5 \mathrm{nmol} / \mathrm{L}$ ) which demonstrated that $\mathrm{I}_{\mathrm{Kr}}$ blockade resulted in significant increase in action potential duration $\left(\mathrm{APD}_{50}: 272 \pm 17 \mathrm{~ms}\right.$ with $0.5 \mathrm{nmol} / \mathrm{L} \mathrm{E} 4031$; $n=5 ;$ p $<0.05$ vs. basal, Fig. 30D). Higher action potential amplitude (APA) and duration (APD) 
observed under basal conditions are indicative of BHM maturation at culture day 29 (Fig. 30D) in comparison to day 22 (Fig. 19).

A

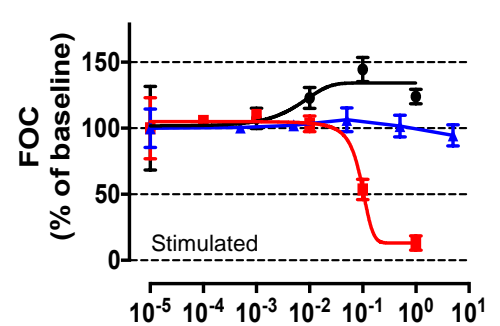

B

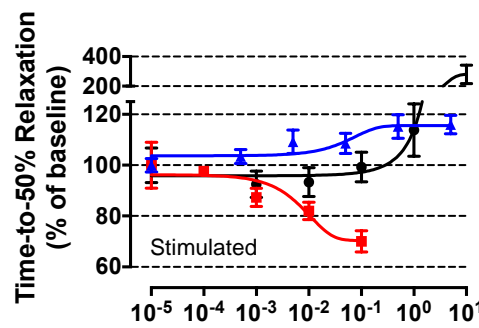

Compound Concentration ( $\mu \mathrm{mol} / \mathrm{L})$

Digitoxin + Verapamil + E4031

D
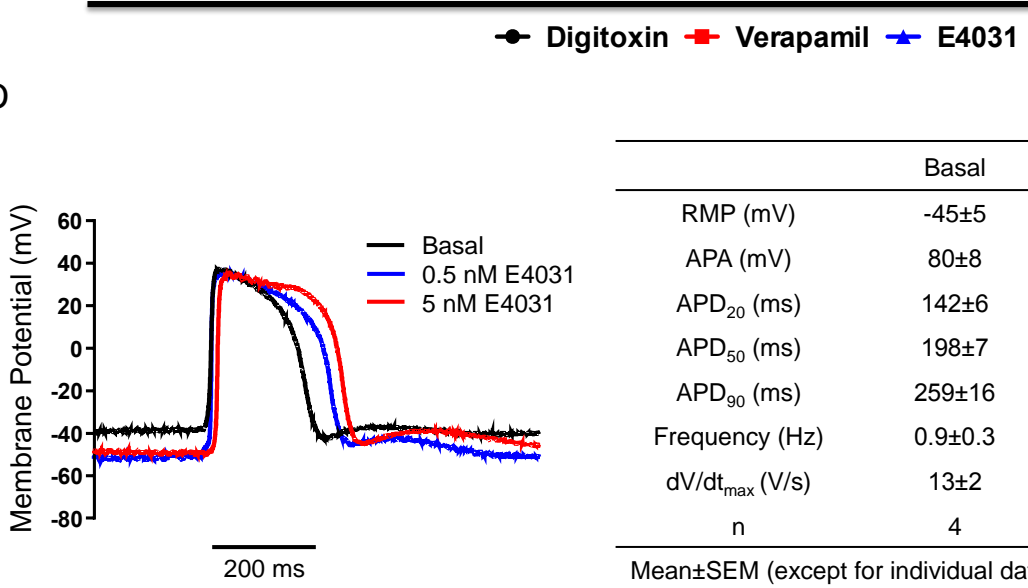

C

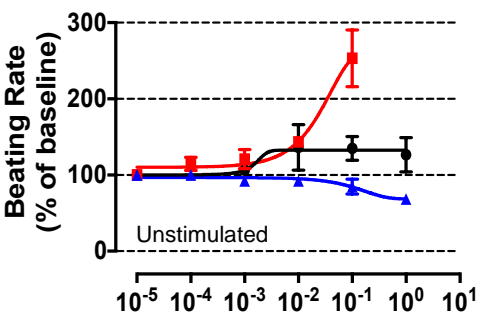

$10^{-5} \quad 10^{-4} 10^{-3} \quad 10^{-2} \quad 10^{-1} \quad 10^{0} 10^{1}$

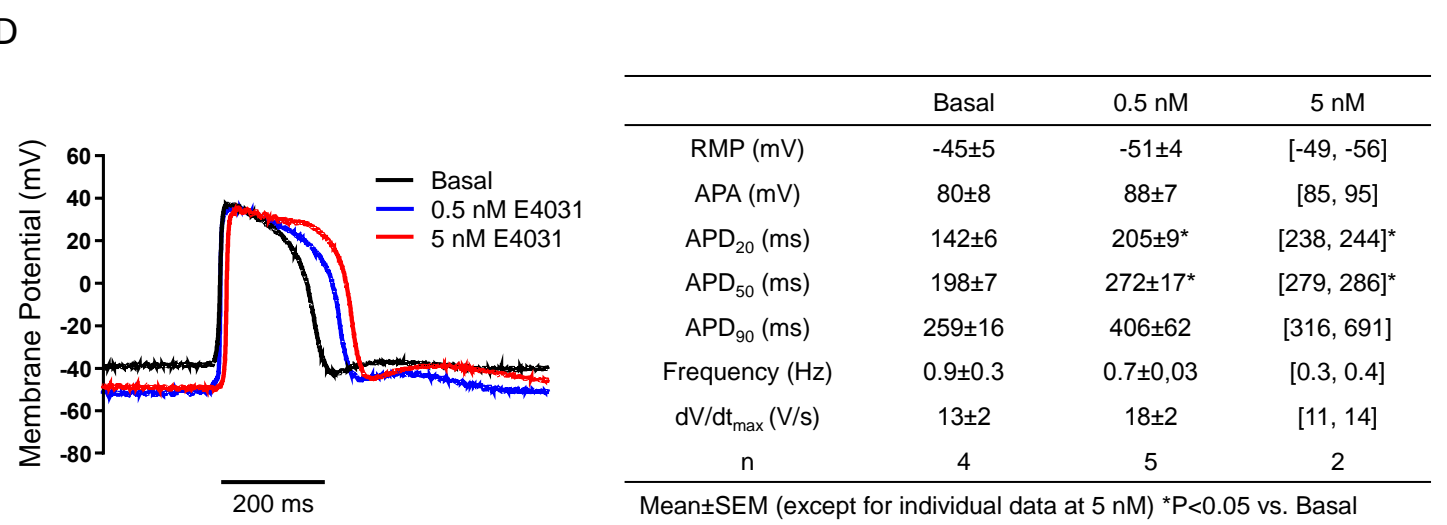

Figure 30: Identification of the biological activity of reference compounds in BHM.

Effects of the positive inotrope digitoxin, the negative inotrope verapamil and the HERG inhibitor E4031 on (A) FOC, (B) time-to-50\% relaxation, and (C) spontaneous beating rate in BHM at culture day 29 ( $n=4-6$ BHMs/group) (D) Impaling electrode measurements of cardiomyocyte action potentials on BHM culture day 29 under HERG blockade with E4031. FOC: force of contraction; RMP: resting membrane potential; APA: action potential amplitude; APD: action potential duration. 


\subsection{Enhancing Maturation of Bioengineered Heart Muscle}

The collected data so far demonstrates that cardiac maturation in BHM is reminiscent of embryonic development. With a focus on BHM culture beyond the established 22-29 days, it was the aim to further delineate specific factors or conditions that may advance maturation of BHM systematically beyond the embryonic state.

Since the time-course experiment confirmed progressive maturation of BHM, we hypothesized that BHM functionality can be further advanced by extended culture for up to 60 days with modulation of TGF $\beta-1$ and insulin signaling as well as the basal medium composition. For these studies, BHMs were generated as described in 2.3.2 and culture was continued with modifications in the designated cardiac maturation phase (cultures days 13-60).

\subsubsection{Variation of TGF $\beta$-1 stimulation during cardiac maturation}

Previously, we observed that TGF $\beta-1$ (1 ng/mL) enhances cardiac maturation in BHM, evidenced by improved contractile function and an increase in MYH7/MYH6 transcript ratio (Hudson et al., in revision). This finding was scrutinized by testing the effect of different TGF $\beta$ 1 concentrations, chronically added to the BHM culture (days 13 to day 22, 29 or 60) on BHM contractile function (Fig. 31A).

Isometric force measurements by BHM culture day 22 revealed an increase in FOC in a concentration dependent manner, with no effect in the $0.3 \mathrm{ng} / \mathrm{mL}$ TGF $\beta-1$ group and markedly enhanced contractility at higher TGF $\beta-1$ concentrations (1-10 ng/mL) (Fig. 31B(i)). BHM cell composition was also affected with a decrease in cardiomyocyte and a concurrent increase in CD90 (fibroblast-like) cell content (Fig. 31B(ii)). Total cell number was not altered (Fig. 31B(iii)), suggesting that TGF $\beta-1$ enhanced FOC per cardiomyocyte in a concentration dependent manner (Fig. 31B(iv)).

Next, we scrutinized whether extended TGF $\beta-1$ (days 13-22 vs. day 13-29 of BHM culture) stimulation would further enhance BHM contractility. Conversely to the positive effect of 
TGF $\beta-1$ addition during culture days 13-22, extended stimulation (days 13-29) resulted in a concentration dependent negative effect on BHM contractility (Fig. 31C(i)). Cardiomyocyte content appeared further decreased (Fig. 31C(ii)) compared to the days 13-22 TGF $\beta-1$ stimulation protocol with however unaltered CD90 cell content. FOC per cardiomyocte was lower in the days 13-29 stimulation protocol as compared to FOC per cardiomyocyte in the days 13-22 stimulation protocol. 


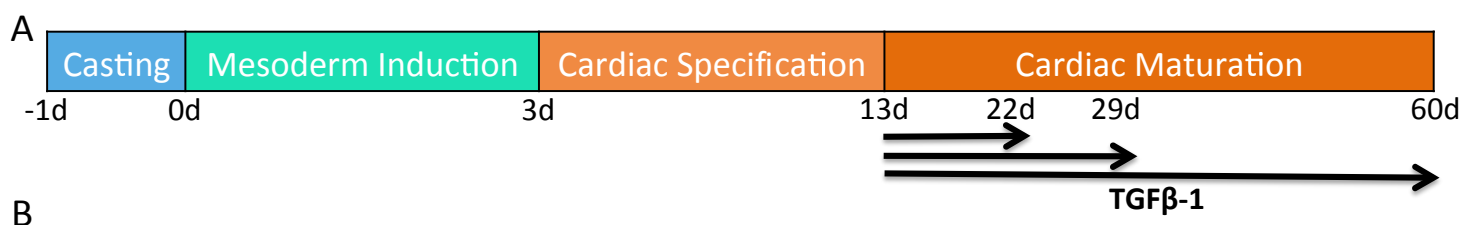

B

(i)

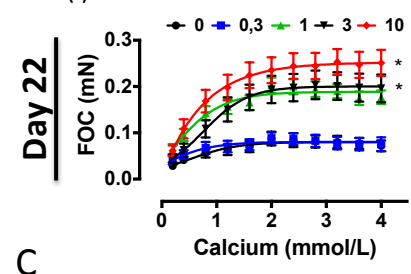

(i)

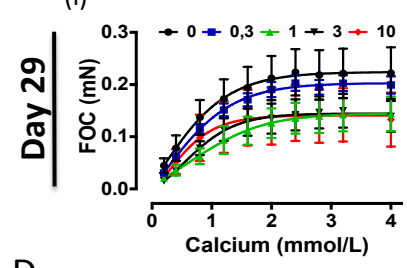

D (i)

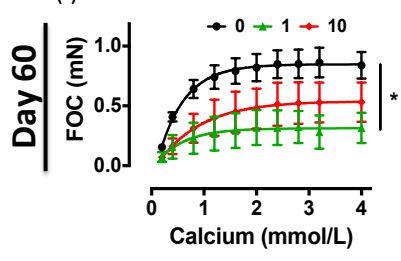

(ii)

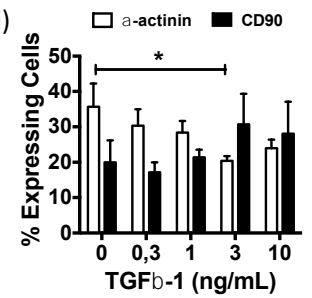

(ii)

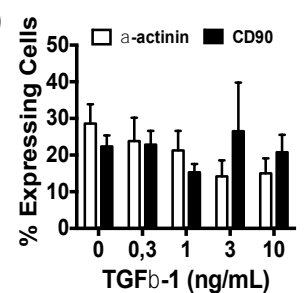

(ii)

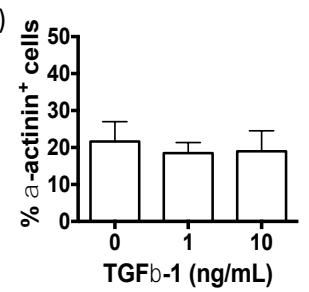

(iii) $\widetilde{\sigma}$

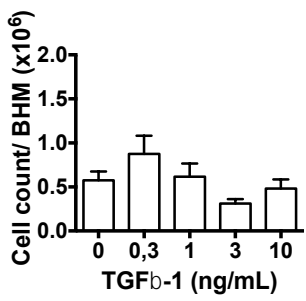

(iii)

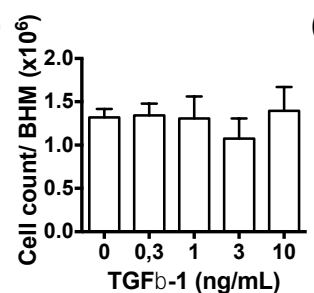

(iii)

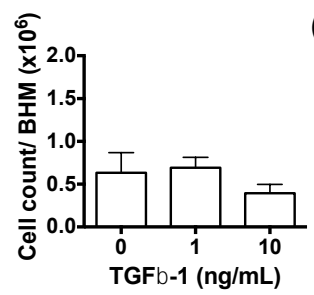

(iv)

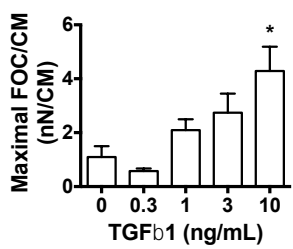

(iv)

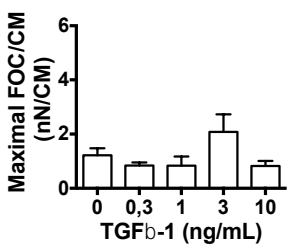

(iv)

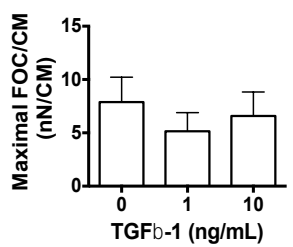

Figure 31: Functional maturation of BHMs by transient TGFß-1 stimulation.

(A) Outline of TGF $\beta-1$ treatment protocol $(0,0.3,1,3$ or $10 \mathrm{ng} / \mathrm{mL})$ in BHMs added with every medium exchange during culture days 13 to day 22, 29 or 60. (B) TGF $\beta-1$ stimulation during culture days 13-22: (i) FOC examined under increasing extracellular calcium concentrations ( $n=9-12 \mathrm{BHMs} /$ concentration). $* P<0.05 \mathrm{vs} .0 \mathrm{ng} / \mathrm{mL}$ by two-way ANOVA with Bonferroni's multiple comparison post-hoc test. (ii) Flow cytometry for cardiomyocyte $(\alpha$ actinin) and stromal cell (CD90) content on culture day 22 ( $n=10 \mathrm{BHMs} /$ concentration). ${ }^{*} P<0.05 \mathrm{vs} .0 \mathrm{ng} / \mathrm{mL}$ by ANOVA with Dunnett's multiple comparison post-hoc test. (iii) Total cell count (x10 $)$ isolated from culture day 22 BHM ( $n=11 \mathrm{BHMs} /$ concentration). (iv) Maximal FOC per BHM cardiomyocyte ( $n=10 \mathrm{BHMs} /$ concentration). $* P$ $<0.05$ vs. $0 \mathrm{ng} / \mathrm{mL}$ by ANOVA with Dunnett's multiple comparison post-hoc test. (C) TGF $\beta-1$ stimulation during culture days 13-29: (i) FOC examined under increasing extracellular calcium concentrations ( $n=8-11$ BHMs/concentration). (ii) Flow cytometry for cardiomyocyte ( $\alpha$-actinin) and stromal cell (CD90) content on culture day 29 ( $n=6 \mathrm{BHMs} /$ concentration). (iii) Total cell count $\left(\mathrm{x} 10^{6}\right)$ isolated from culture day $29 \mathrm{BHM}(n=6$ BHMs/concentration). (iv) Maximal FOC per BHM cardiomyocyte ( $n=6 \mathrm{BHMs} /$ concentration). (D) TGF $\beta-1(0,1$ or $10 \mathrm{ng} / \mathrm{mL}$ ) stimulation during culture days 13-60: (i) FOC examined under increasing extracellular calcium concentrations ( $n=4 \mathrm{BHMs} /$ concentration). ${ }^{*} P<0.05 \mathrm{vs} .0 \mathrm{ng} / \mathrm{mL}$ by two-way ANOVA with Bonferroni's multiple comparison post-hoc test. (ii) Flow cytometry for cardiomyocyte ( $\alpha$-actinin) content on culture day 60 ( $n=4 \mathrm{BHMs} /$ concentration). (iii) Total cell count $\left(\mathrm{x} 10^{6}\right)$ isolated from culture day 60 BHM $(n=4$ BHMs/concentration). (iv) Maximal FOC per BHM cardiomyocyte ( $n=4$ BHMs/concentration). TGF $\beta-1$ : transforming growth factor $\beta-1$; BHM: bioengineered heart muscle; CM: cardiomyocyte; FOC: force of contraction; CD90: Thy-1 cell surface antigen; d: days.

To further define the effect of TGF $\beta-1$ on BHM maturation, we next tested whether long-term stimulation from culture day 13 to culture day 60 with 1 or $10 \mathrm{ng} / \mathrm{mL}$ TGF $\beta-1$ would have a beneficial or detrimental effect on BHM contractility (Fig. 31D(i)). Surprisingly and in contrast 
to our earlier short (day 13-22) and intermediate (day 13-29) term studies, we observed the best contractile performance in BHM not treated with TGF $\beta-1$ at all. Cardiomyocyte content was in all conditions at $\sim 20 \%$ (Fig. 31D(ii)) with a similar BHM cell count as observed on culture day 22 and a $\sim 50 \%$ reduced cell count as compared to culture day 29 (Fig. 31D(iii)). FOC per cardiomyocyte was unaffected (Fig. 31D(iv)). On the morphological level, TGF $\beta-1$ treated tissues were significantly thinner as indicated by cross sectional area analyses (Fig. 32A), which is consistent with role of TGF $\beta-1$ in extracellular matrix production.

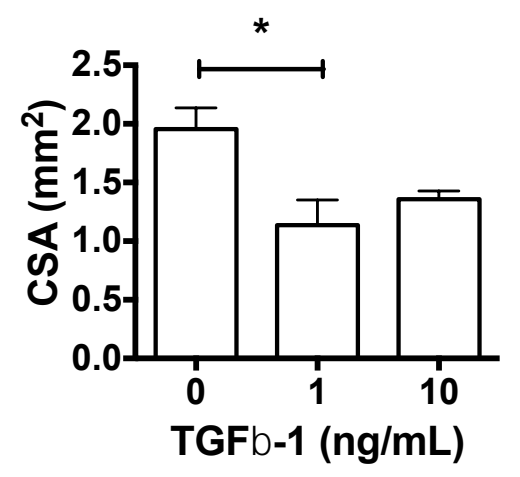

Figure 32: Cross sectional area of BHM under TGF $\beta-1$ stimulation.

Cross sectional area (CSA) of BHMs treated with TGF $\beta-1(0,1$ or $10 \mathrm{ng} / \mathrm{mL})$ during culture days $13-60(n=4$ BHMs/concentration). $* P<0.05$ vs. $0 \mathrm{ng} / \mathrm{mL}$ by one-way ANOVA with Tukey's multiple comparison post-hoc test. TGF $\beta-1$ : transforming growth factor $\beta-1$; CSA: cross sectional area; FOC: force of contraction.

Collectively, this series of experiments indicated the modulatory role of TGF $\beta-1$ in early BHM maturation. This effect appears to be balanced out in long-term cultures potentially by intrinsic TGF $\beta-1$ release. A pilot experiment with cultures for 29 and 60 days of transient TGF $\beta-1$ (10 $\mathrm{ng} / \mathrm{mL}$ ) stimulation only during culture days 13-22 was in agreement with this hypothesis (Fig. 33). It also suggested that TGF $\beta-1$ supplementation at the early maturation phase may stabilize long-term outcome. 


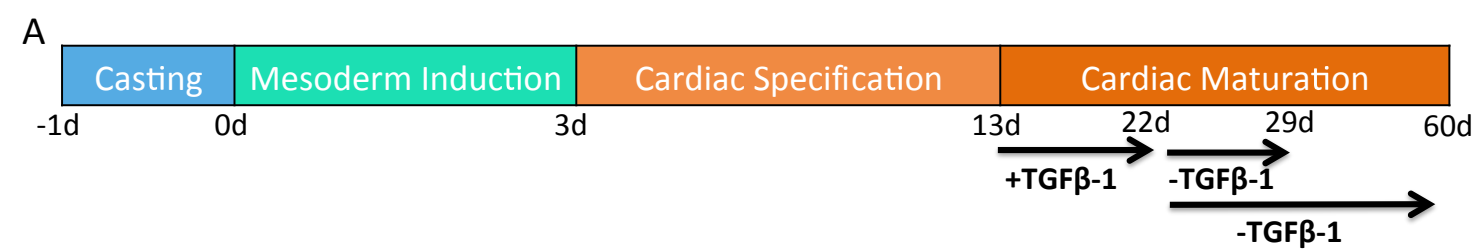

B
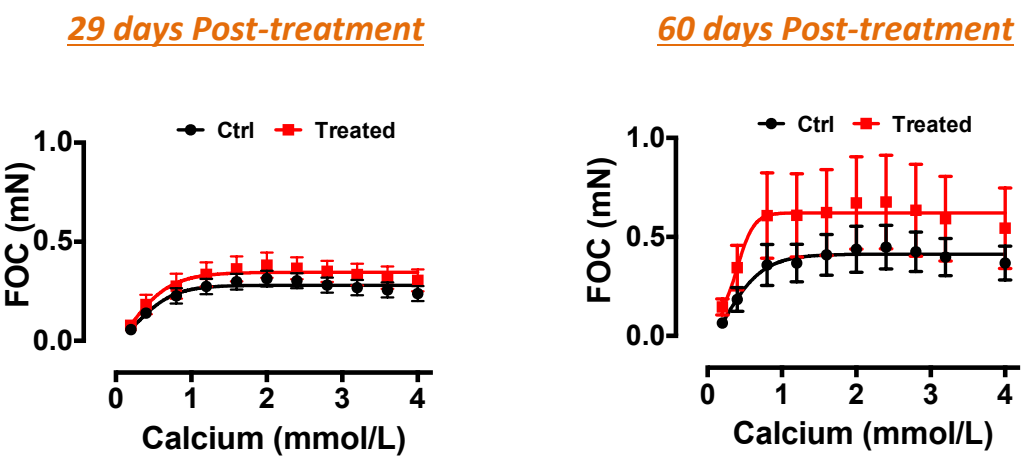

Figure 33: Stabilization of long-term outcome by transient TGF $\beta-1$ supplementation.

(A) Outline of TGF $\beta-1(10 \mathrm{ng} / \mathrm{mL})$ temporary treatment protocol (from day 13 to day 22). (B) FOC examined under increasing extracellular calcium concentrations measured under isometric conditions in 29 and 60 days culture BHM. ( $n=3$ BHMs/concentration). TGF $\beta-1$ : transforming growth factor $\beta-1$; BHM: bioengineered heart muscle; FOC: force of contraction, d: days; Ctrl: control.

\subsubsection{Omission of insulin during cardiac maturation}

Insulin and insulin-like growth factor (IGF) are implicated in cardiomyocyte proliferation and hypertrophic growth in vivo (mouse embryos) (Li et al., 2011) and in vitro (hESC derivedcardiomyocytes) (McDevitt et al., 2005). From previous studies in the lab we have learned that omitting insulin during mesoderm induction and cardiac specification (BHM culture days 0-13) leads to impaired cardiogenesis (Hudson et al., in revision). Thus, we tested whether insulin removal during "cardiac maturation" (BHM culture days 13 to 22, 29 or 60) would enhance or diminish BHM function. Isometric force measurements in these different groups did not identify differences in FOC (Fig. 34B/C/D (i)) despite a significant decrease in cardiomyocyte content in the absence of insulin (Fig. 34B/C/D (ii)). Total BHM cell content varied among these different groups depending on the BHM culture day with an overall increase by culture day 60 in the absence of insulin (Fig. 34B/C/D (iii)). Force per cardiomyocyte was markedly enhanced in the absence of insulin during short (day 13-22) and intermediate (day 13-29) term studies, but 
similar in day 60 insulin treated and day 13-60 without insulin BHMs (Fig. 34B/C/D (iv)). Based on this observation it may be reasonable to withdraw insulin during cardiac maturation.

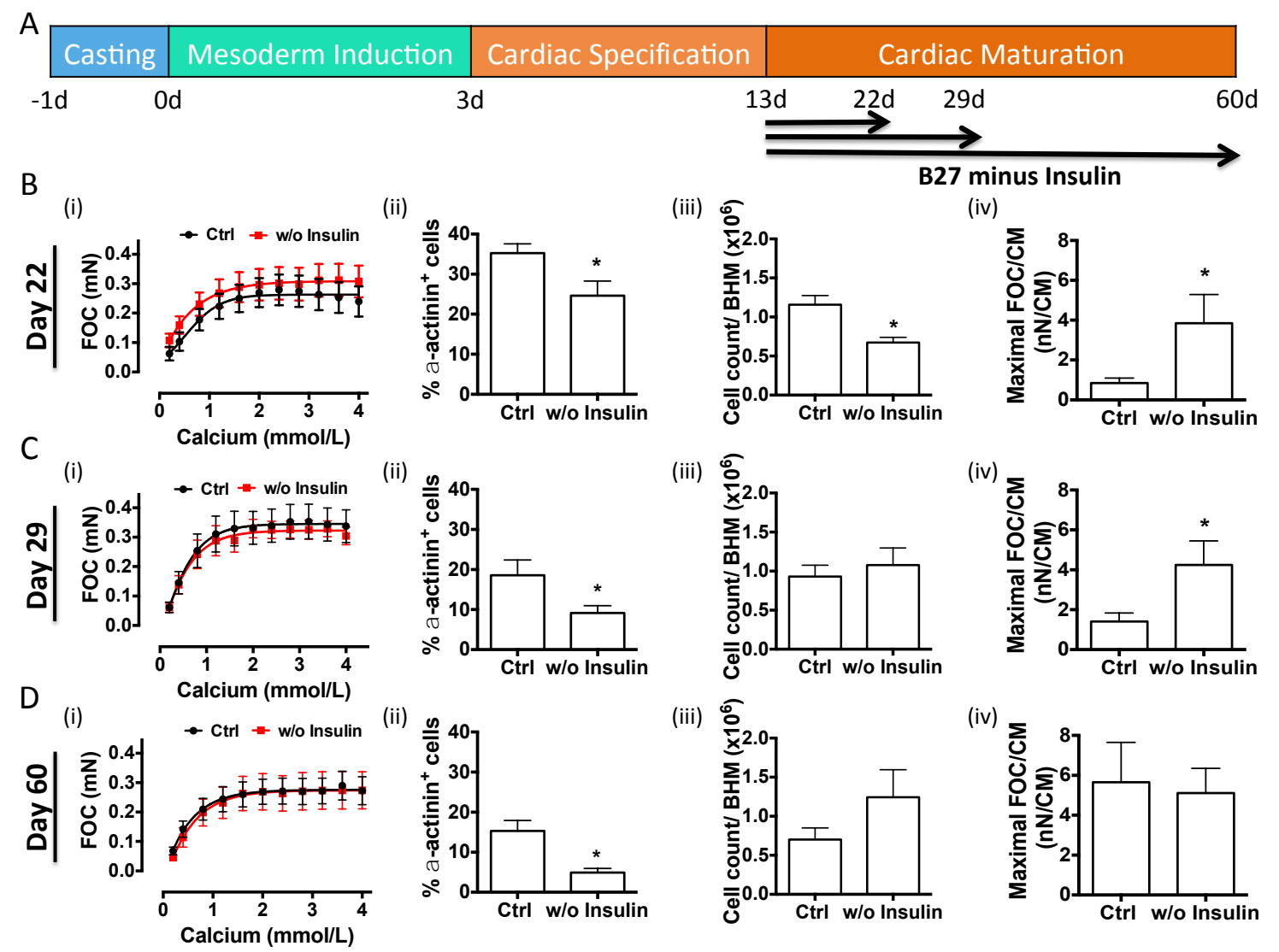

Figure 34: Effect of insulin on BHM function.

(A) Outline of insulin removal protocol in BHMs with every medium exchange during culture days 13-22, 13-29 or 13-60. (B) Insulin removal during culture days 13-22: (i) FOC examined under increasing extracellular calcium concentrations ( $n=9 \mathrm{BHMs} /$ group). (ii) Flow cytometry for cardiomyocyte ( $\alpha$-actinin) content on culture day 22 ( $n=8 \mathrm{BHMs}$ /group). $* P<0.05$ vs. Ctrl by unpaired t-test. (iii) Total cell count $\left(\mathrm{x} 10^{6}\right)$ isolated from culture day 22 BHM ( $n=9$ BHMs/group). $* P<0.05$ vs. Ctrl by unpaired t-test. (iv) Maximal FOC per BHM cardiomyocyte $(n=7$ BHMs/group). ${ }^{*} P<0.05$ vs. Ctrl by unpaired t-test. (C) Insulin removal during culture days 13-29: (i) FOC examined under increasing extracellular calcium concentrations ( $n=8 \mathrm{BHMs} /$ group). (ii) Flow cytometry for cardiomyocyte ( $\alpha$-actinin) content on culture day 29 ( $n=7$ BHMs/group). $* P<0.05$ vs. Ctrl by unpaired t-test. (iii) Total cell count $\left(\times 10^{6}\right)$ isolated from culture day 29 BHM ( $n=7$ BHMs/group). (iv) Maximal FOC per BHM cardiomyocyte ( $n=7$ BHMs/group). ${ }^{*} P<0.05$ vs. Ctrl by unpaired t-test. (D) Insulin removal during culture days 13-60: (i) FOC examined under increasing extracellular calcium concentrations ( $n=7$ BHMs/group). (ii) Flow cytometry for cardiomyocyte ( $\alpha$-actinin) content on culture day 60 ( $n=6$ BHMs/group). ${ }^{*} P<0.05$ vs. Ctrl by unpaired t-test. (iii) Total cell count $\left(\times 10^{6}\right)$ isolated from culture day 60 BHM ( $n=6$ BHMs/group). (iv) Maximal FOC per BHM cardiomyocyte ( $n=6 \mathrm{BHMs} /$ group). Ctrl: control; w/o Insulin: B27 minus insulin; BHM: bioengineered heart muscle; CM: cardiomyocyte; FOC: force of contraction; d: days. 


\subsubsection{Optimization of basal medium in long-term cultures}

RPMI1640 (composition in Appendix) has been described as the most commonly used medium in stem cell cultures and cardiomyocyte differentiation; it was also used in our original BHM protocol. In long term BHM cultures (>29 days) we frequently observed culture medium precipitations, which may be attributed to the high phosphate content and thus formation of insoluble calcium phosphate. We thus tested Iscove's medium (composition in Appendix) as an alternative with similar amino acid and vitamin components, but more suitable calcium levels (1.2 mmol/L) and lower phosphate concentration (Fig. 35A).
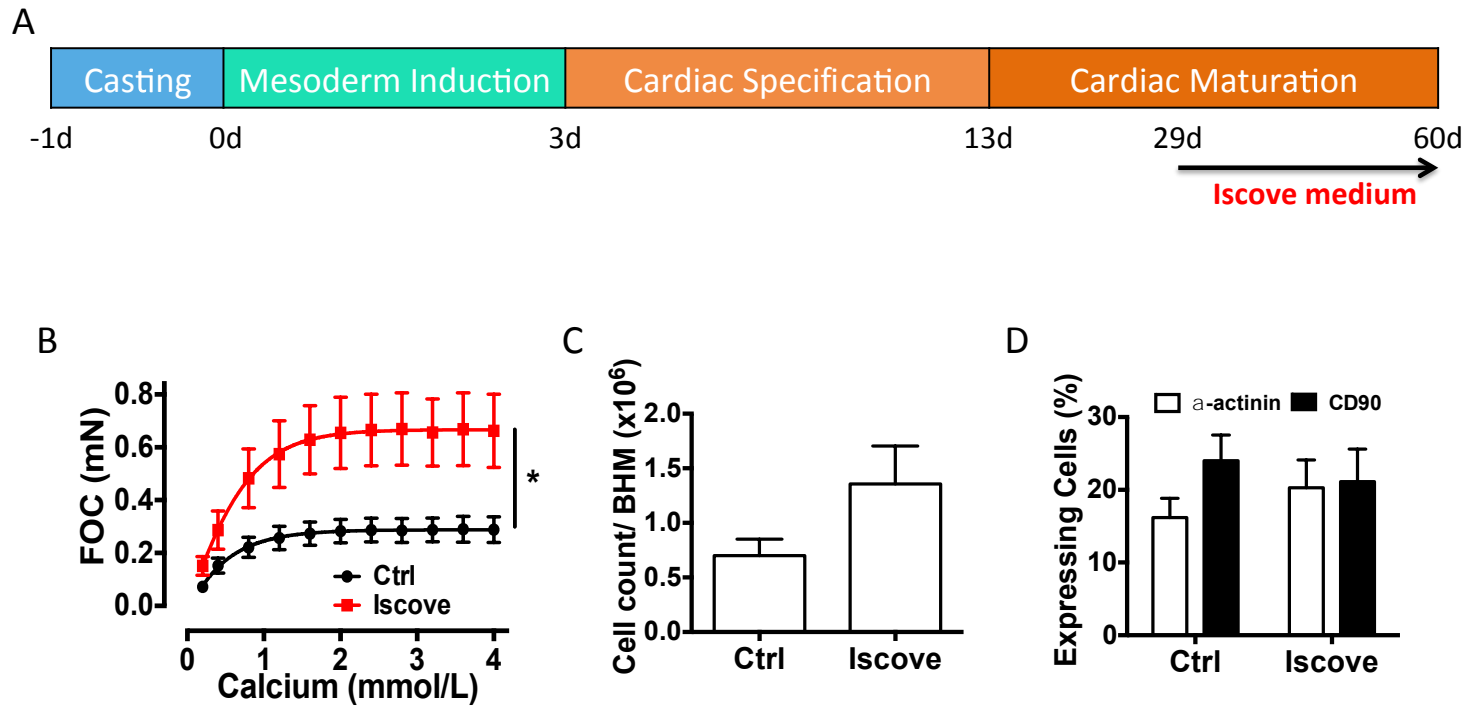

Figure 35: Consequences of basal medium on BHM function and cellularity.

(A) Outline of the BHM cultivation protocol with RPMI or in Iscove's medium during culture days 29-60. (B) FOC examined under increasing extracellular calcium concentrations measured under isometric conditions by culture day 60 ( $n=11 \mathrm{BHMs}$ /group). * $P<0.05$ by two-way ANOVA with Bonferroni's multiple comparison post-hoc test.

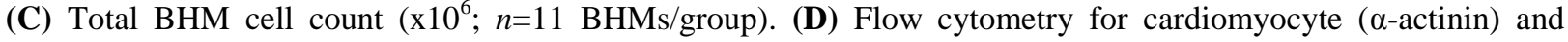
stromal cells (CD90) on culture day 60 ( $n=11 \mathrm{BHMs/group).} \mathrm{Ctrl:} \mathrm{control;} \mathrm{BHM:} \mathrm{bioengineered} \mathrm{heart} \mathrm{muscle;}$ CD90: Thy-1 cell surface antigen; d: days.

A medium change from RPMI to Iscove's between culture day 29 and 60 resulted in markedly higher contractile forces (Fig. 35B). Total cell count was higher (not significant; Fig. 35C) and cell composition remained unaffected in the Iscove's group (Fig. 35D). 
In summary, BHM maturation is a continuous process. A surprising observation was the decrease in cardiomyocyte content despite enhanced function. Addition of TGF $\beta-1$ (10 ng/mL) within a narrow time window (culture days 13-22), withdrawal of insulin during cardiac maturation (day 13 onwards) and replacement of RPMI with Iscove's basal medium in longterm cultures (day 29 onwards) were identified as useful modifications for robust BHM maturation.

\subsection{Replacement of BMP4 by a Small Molecule Mimic during Mesoderm Induction}

The BHM protocol was developed for robust application to multiple human PSC lines (Hudson et al., in revision). We however argued that replacing the protein growth factors with small molecules would further stabilize the BHM production process and focused on one of the growth factors, namely BMP4, with a pivotal role in mesoderm induction and known batch-tobatch variation of its biological activity (Burridge et al., 2011; Hudson et al., 2012; Laflamme et al., 2007; Yang et al., 2008; Yao et al., 2006). The first step was to re-evaluate the optimal time window and concentration of BMP4 for mesoderm induction. This was followed by the screening for small molecule derivatives of the chalcone family in monolayer and BHM differentiation.

\subsubsection{Re-evaluation of the role of BMP4 for mesoderm induction in monolayer culture}

The evaluation of the BMP4 effect on mesoderm induction involved the testing of 0,5 and 30 $\mathrm{ng} / \mathrm{mL}$ at different time-points of mesoderm induction (day 0 [24 hours]; day 0-1 [48 hours]; day 0-2 [72 hours]) with subsequent culture under standard conditions until day 22 (Fig. 36B). $\alpha$-Actinin protein expression was assessed by flow cytometry to evaluate cardiomyocyte differentiation efficiency under the different conditions. Consistent with previous results (Hudson et al., in revision), $5 \mathrm{ng} / \mathrm{mL}$ BMP4 for at least 48 hours was optimal for the induction of cardiac mesoderm (Fig. 36B). Morphologically, BMP4 supplementation (5 ng/mL) for 72 hours resulted in more synchronous beating activity. Subsequently, we defined the concentration dependent effect of BMP4 on concurrent cardiomyocyte and fibroblast-like cell (CD90 $)$ induction capacity (Fig 36C). The cardiomyocyte and $\mathrm{CD}^{+} 0^{+}$cell content populations were low 
(19 $\pm 3 \%$ and $8 \pm 1 \%$, respectively; $n=3)$ in the absence of BMP4. With the addition of $5 \mathrm{ng} / \mathrm{ml}$ $\mathrm{BMP} 4$, the population of both $\alpha$-actinin and $\mathrm{CD}^{+} 0^{+}$cells increased markedly to $54 \pm 2 \%$ and $20 \pm 5 \%$, respectively $(n=4)$. Upon a further increase of BMP4, cardiomyocyte number decreased significantly $(12 \pm 3 \%)$, while the percentage of stromal cells doubled $(39 \pm 4 \% ; n=4)$.

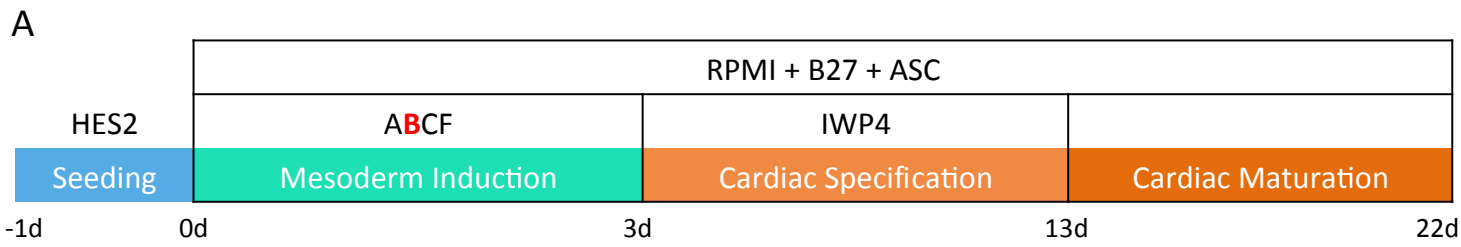

B

\begin{tabular}{|c|c|c|c|}
\hline BMP4 (ng/mL) & Day 0 & Day 1 & Day 2 \\
\hline 0 & - & - & - \\
\hline \multirow{2}{*}{5} & + & + & - \\
\cline { 2 - 4 } & + & + & + \\
\hline \multirow{3}{*}{30} & + & - & - \\
\cline { 2 - 4 } & + & + & - \\
\cline { 2 - 4 } & + & + & + \\
\hline
\end{tabular}

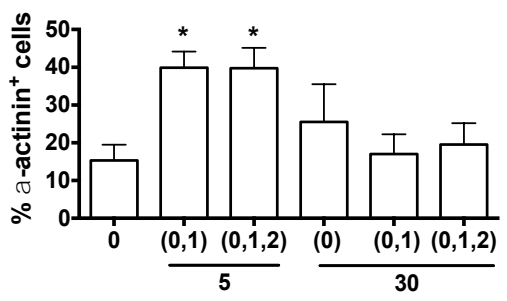

C

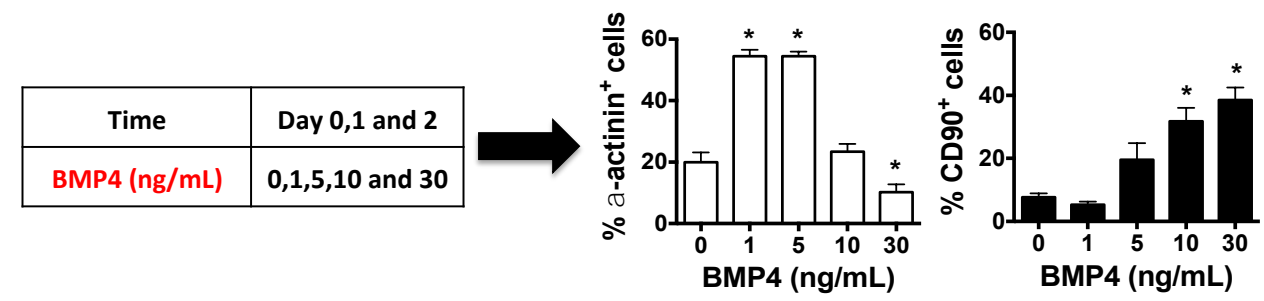

Figure 36: Effects of BMP4 on mesoderm induction in monolayer cultures.

(A) Outline of the differentiation protocol (B in red indicates the time window for BMP4 supplementation). (B) Overview of the experimental conditions tested and the resulting effects on cardiomyocyte differentiation assessed by flow cytometry for $\alpha$-actinin at monolayer culture day 22 ( $n=4$ samples/group). ${ }^{*} P<0.05 \mathrm{vs} .0 \mathrm{ng} / \mathrm{mL}$ of BMP4 by one-way ANOVA with Dunnett's multiple comparison post-hoc test. (C) Overview of the experimental conditions tested and the resulting effects on cardiomyocyte and fibroblast-like cell differentiation assessed by flow cytometry for $\alpha$-actinin and CD90 at monolayer culture day 22 ( $n=4$ samples/concentration). ${ }^{*} P<0.05$ vs. 0 $\mathrm{ng} / \mathrm{mL}$ of BMP4 by one-way ANOVA with Dunnett's multiple comparison post-hoc test. HES2: human embryonic stem cell line 2; A: activin-A; B: bone morphogenetic protein 4; C: CHIR99021; F: fibroblast growth factor-2; IWP4: inhibitor of Wnt production 4; RPMI: Rosewell Park Memorial Institute basal medium; ASC: 1-ascorbic acid; CD90: Thy-1 cell surface antigen; d: days. 


\subsubsection{Re-evaluation of the role of BMP4 for mesoderm induction in BHM}

To study the effect of BMP4 on BHM development and function, we added $0,1,5,10$ or 30 $\mathrm{ng} / \mathrm{mL}$ BMP4 during mesoderm induction (Fig. 37A). Isometric force measurements at day 22 revealed impaired functionality in the absence of BMP4 (Fig. 37B) associated with low cardiomyocyte content $\left(3 \pm 1 \% \quad \alpha\right.$-actinin ${ }^{+}$cells; $n=6-8$; Fig. 37C). Conversely, BHM demonstrated robust contractility upon addition of $5 \mathrm{ng} / \mathrm{mL}$ BMP4 during mesoderm induction (Fig. 37B) with a concurrently increased cardiomyocyte content (56 $\pm 3 \%$; $n=6-8$; Fig. 37C). Cardiomyocyte size was enhanced by 1.5 -fold upon BMP4 addition even at low concentrations (Fig. 37D). Interestingly, higher concentrations of BMP4 (30 ng/mL) resulted in impaired BHM function and cardiac output ( $7 \pm 1 \%$ of $\alpha$-actinin ${ }^{+}$cells; $n=6-8$; Fig. 37C) with a 2 -fold increase in $\mathrm{CD} 90^{+}$cells $(63 \pm 3$ vs $31 \pm 3 \%$ in 30 vs. $0 \mathrm{ng} / \mathrm{mL}$ BMP4 treated BHM, respectively; $n=6-8$; p $<0.01$; Fig. 37C). Collectively, this data confirmed the important role of mesoderm induction by BMP4 for the differentiation of HES into cardiomyocyte in monolayer and BHM culture. 
A

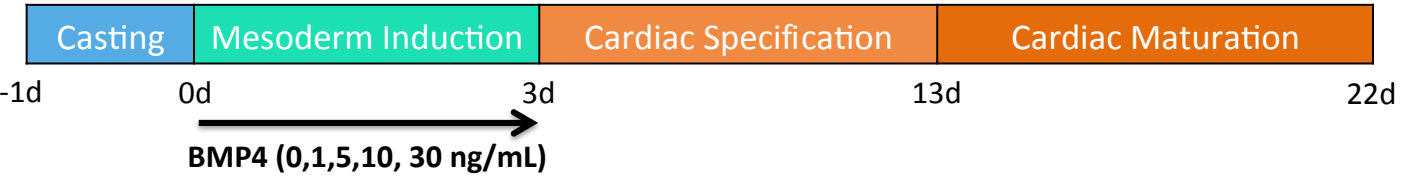

B

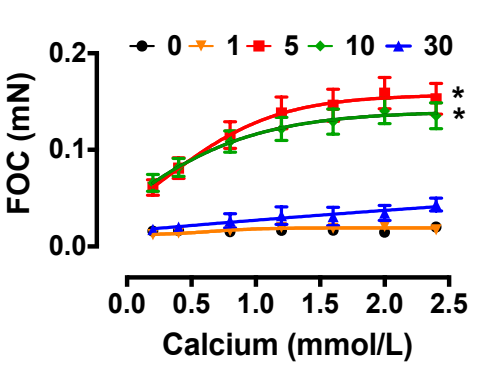

C

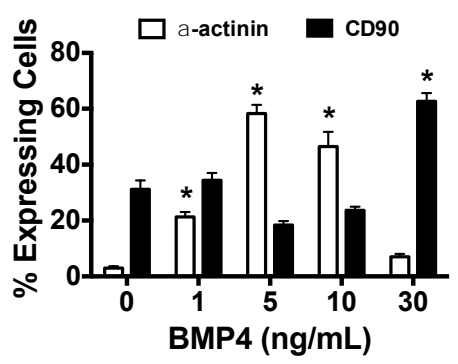

D

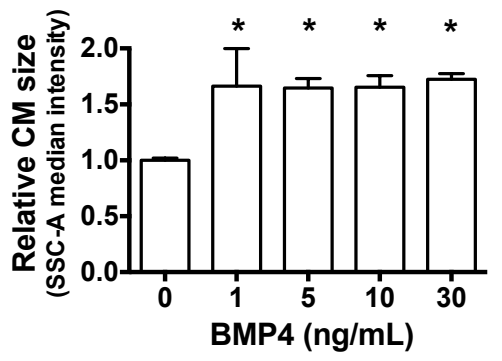

Figure 37: Effects of BMP4 on mesoderm induction in BHM.

(A) Outline of BMP4 $(0,1,5,10$ and $30 \mathrm{ng} / \mathrm{mL})$ stimulation protocol during mesoderm induction in BHM. (B) Inotropic response to increasing extracellular calcium measured under isometric conditions in BHMs treated with the indicated BMP4 concentrations for mesoderm induction ( $n=6-8 \mathrm{BHMs} /$ concentration). $* P<0.05 \mathrm{vs} .0 \mathrm{ng} / \mathrm{mL}$ of BMP4 by two-way ANOVA with Bonferroni's multiple comparison post-hoc test (C) Flow cytometry analysis of cardiomyocyte ( $\alpha$-actinin) and stromal cells (CD90) content in day 22 BHM treated with the indicated BMP4 concentrations during mesoderm induction ( $n=6-8 \mathrm{BHMs} /$ concentration). ${ }^{*} P<0.05 \mathrm{vs.} 0 \mathrm{ng} / \mathrm{mL}$ of BMP4 by oneway ANOVA with Dunnett's multiple comparison post-hoc test. (D) Cardiomyocyte (CM) size (side scatter median intensity normalized to $0 \mathrm{ng} / \mathrm{mL}$ BMP4) assessed by flow cytometry ( $n=6-8 \mathrm{BHMs} /$ concentration). $* P<0.05$ vs. 0 $\mathrm{ng} / \mathrm{mL}$ BMP4 by ANOVA with Dunnett's multiple comparison post-hoc test. BMP4: bone morphogenetic protein 4; CD90: Thy-1 cell surface antigen; CM: cardiomyocyte; FOC: force of contraction; SSC-A: side scatter median intensity; d: days.

\subsubsection{Effect of BMP4 on gene expression profiles during BHM development}

qPCR analyses were performed to elucidate the role of BMP signaling for mesoderm induction and subsequent cardiac specification and maturation on the molecular level (Fig. 38A). In-line with the observations above, we noted that BMP4 reduced pluripotent marker OCT4 expression and enhanced MESP1 expression at day 3 as a sign for mesodermal commitment (Fig. 38B). $N K X 2-5$ and ISLI were enhanced by day 8 followed by an increase in the late cardiac marker MYL2 at day 22. The latter effects were more pronounced in the $5 \mathrm{ng} / \mathrm{mL}$ BMP4 group. The failure to enhance $N K X 2-5$ in the presence of $30 \mathrm{ng} / \mathrm{mL}$ BMP4 was in line with the low cardiomyocyte content by day $22\left(7 \pm 1 \%\right.$ of $\alpha$-actinin ${ }^{+}$cells, Fig. 37C) and the failure of BHM to contract (Fig. 37B). Collectively, these data confirm the essential role of BMP4 during the 
narrow time window of mesoderm induction for long-term outcome in monolayer and BHM differentiation.
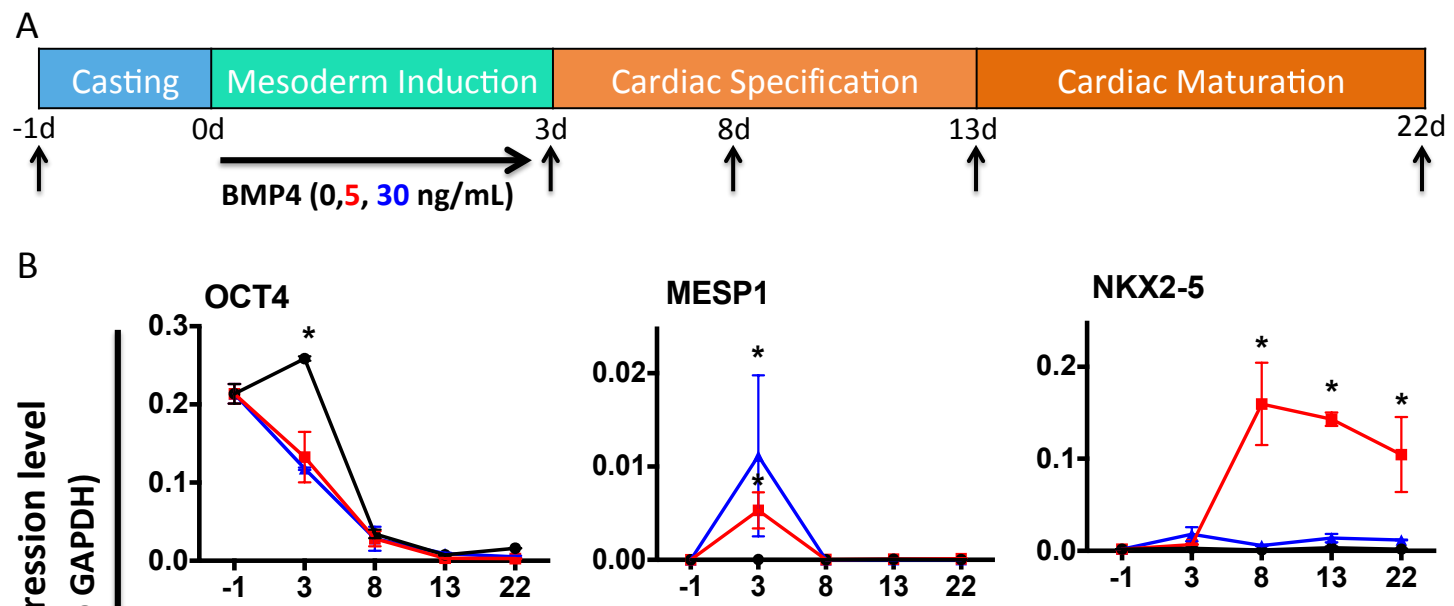

SL1
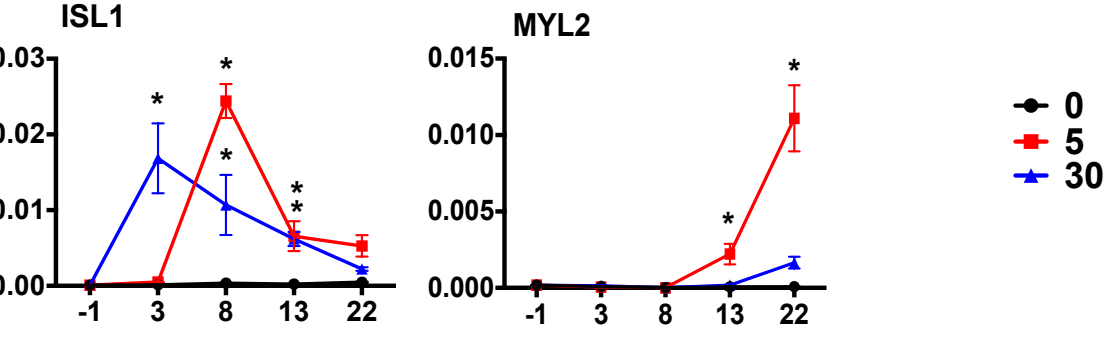

BHM culture days

Figure 38: Molecular consequences of BMP4 stimulation during mesoderm induction in BHM.

(A) Outline of the BMP4 stimulation and culture protocol. (B) Relative gene expression of pluripotent marker OCT4, mesodermal marker MESP1, early cardiac markers NKX2-5 and ISL1 as well as the late cardiac marker MYL2 using qPCR. All data were normalized to GAPDH transcript abundance ( $n=3 \mathrm{BHMs} /$ time point). ${ }^{*} P<0.05$ vs. $0 \mathrm{ng} / \mathrm{mL}$ BMP4 by two-way ANOVA with Dunnett's multiple comparison post-hoc test. BMP4: bone morphogenetic protein 4; d: days; OCT4: octamer-binding transcription factor 4; MESP1: mesoderm posterior basic helix-loop-helix transcription factor 1; NKX2-5: NK2 transcription factor related locus 5; ISL1: islet 1; MYL2: myosin light chain 2; GAPDH: glyceraldehyde-3-phosphate-dehydrogenase; BHM: bioengineered heart muscle.

\subsubsection{Chalcones as potential small molecule activators of BMP signaling}

In a recent screen for small molecule activators of BMP signaling using luciferase assay under the Id1 promoter, four hit compounds from the family of flavonoids induced luciferase activity 
greater than 50\% relative to BMP4 (Vrijens et al., 2013). Two compounds were chalcones (isoliquiritigenin and 4'-hydroxychalcone) and the other two were flavones (apigenin and diosmetin). Only the former compounds activated BMP signaling via the phosphorylation of SMAD $1 / 5$ as well as the expression of Id1 target genes in a concentration dependent manner. Based on these findings, we first investigated whether the two chalcones reported previously (4'-hydroxychalcone and isoliquiritigenin) could be used as BMP4 mimics in cardiac differentiation. We further performed an additional small scale efficacy screen with other available chalcones (cardamonin and hespiridine) and 15 in-house synthesized compounds (listed in Appendix) kindly provided by Prof. Dr. Dr. L. F. Tietze (Institute of Organic and Biomolecular Chemistry; Georg-August-Universität Göttingen).

\subsubsection{Screen for biological activity of commercially available chalcones and newly synthesized chalcones}

4'-hydroxychalcone and isoliquiritigenin were added at a concentration range from 0.0001 to $100 \mu \mathrm{mol} / \mathrm{L}$ during mesoderm induction instead of BMP4 in a monolayer HES2 differentiation format (Fig. 39A). DMSO (0.001\%) was added to the negative and positive (5 ng/mL BMP4) control to ensure comparability of the result (all chalcones were solubilized in DMSO). Cells were harvested at day 22 and analysed for the expression of $\alpha$-actinin cardiac marker using flow cytometry (Fig. 39B). Increasing concentration of 4'-hydroxychalcone led to a gradual increase in the cardiomyocyte population which peaked at $10 \mu \mathrm{mol} / \mathrm{L}$ with comparable result to BMP4 induction (\% $\alpha$-actinin ${ }^{+}$cells: $43 \pm 5 \%$ for $10 \mu \mathrm{mol} / \mathrm{L} 4$ '-hydroxychalcone vs. $40 \pm 3 \%$ for $5 \mathrm{ng} / \mathrm{mL}$ BMP4; n=4-9; Fig. 39B). Isoliquiritigenin, on the other hand, demonstrated a biphasic effect on cardiac output which decreased first from $34 \pm 5 \%$ at $0.0001 \mu \mathrm{mol} / \mathrm{L}$ to $8 \pm 2 \%$ at $0.01 \mu \mathrm{mol} / \mathrm{L}$ and then increased again to $39 \pm 3 \%$ at $10 \mu \mathrm{mol} / \mathrm{L}$. Note that both molecules induced cell death and consequential drop in cardiac cell population at $100 \mu \mathrm{mol} / \mathrm{L}$ indicating substance toxicity. In short, chalcones represent potential replacements of BMP4 during mesoderm induction with optimal concentration of $10 \mu \mathrm{mol} / \mathrm{L}$. This concentration was used as a reference for the subsequent small scale screen for BMP4 mimics. 


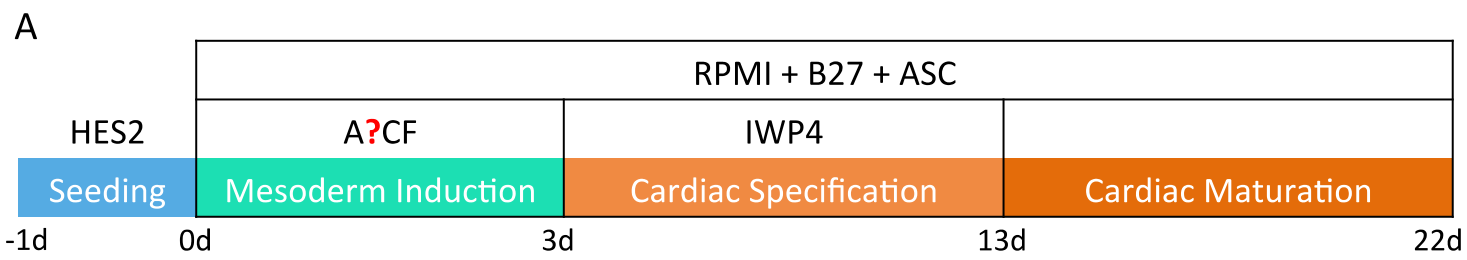

B

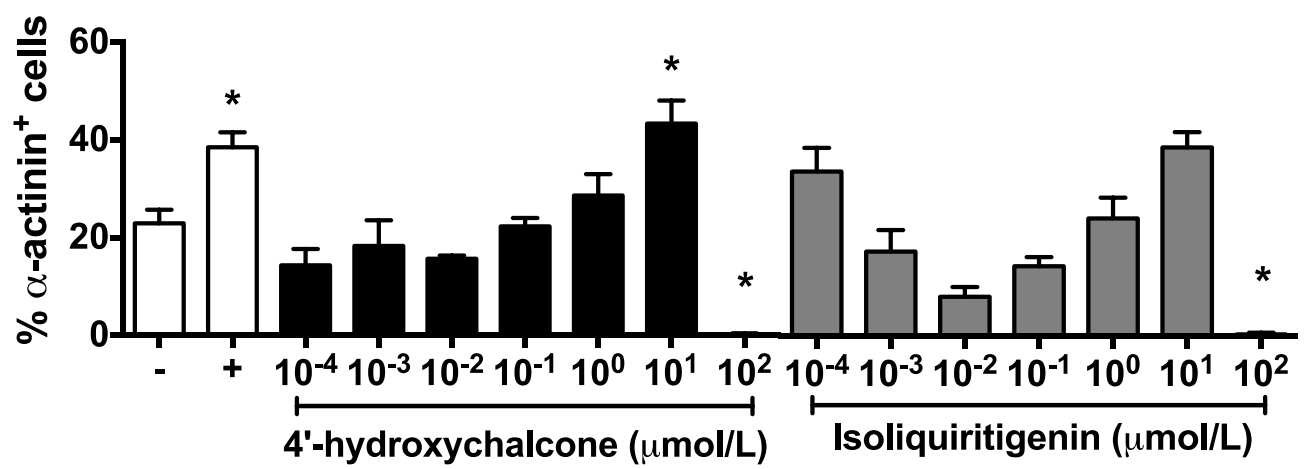

Figure 39: Preliminary screen of chalcones as BMP4 mimics for mesoderm induction.

(A) Outline of the experimental protocol with the use of 4'-hydroxychalcone and isoliquiritigenin as BMP4 replacements during mesoderm induction in a monolayer culture format. (B) Flow cytometry analysis of cardiomyocyte content ( $\alpha$-actinin) on culture day 22 (n=4-9 samples/concentration). Note that either chalcone exhibited cytotoxic effects at $100 \mu \mathrm{mol} / \mathrm{L}$. All compounds were solubilized in DMSO (100\%). DMSO alone served as negative (-) control. $5 \mathrm{ng} / \mathrm{mL}$ BMP4 with $0.001 \%$ DMSO served as positive (+) control. $* P<0.05$ vs. negative control by ANOVA with Dunnett's multiple comparison post-hoc test. HES2: human embryonic stem cell line 2; A: activin-A; B: bone morphogenetic protein 4; C: CHIR99021; F: fibroblast growth factor-2; IWP4: inhibitor of Wnt production-4; RPMI: Rosewell Park Memorial Institute basal medium; ASC: 1-ascorbic acid; d: days.

Next, other commercially available chalcones such as cardamonin and hesperidine as well as 15 newly synthesized chalcones (refer to Appendix for chemical structures) were tested at 10 $\mu \mathrm{mol} / \mathrm{L}$ for their capacity to induce cardiac differentiation when added for 72 hours during mesoderm induction (Fig. 40). Only isoliquiritigenin (in contrast to our first screen Fig. 39B) and hesperidin showed no effect on cardiac differentiation (Fig. 40). All other compounds including the newly snythesized compounds appeared to mimic BMP4 activity. 


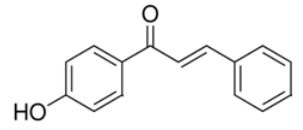

A: 4'-hydroxychalcone<smiles>COc1cc(O)cc(O)c1C(=O)/C=C/c1ccccc1</smiles>

C: Cardamonin

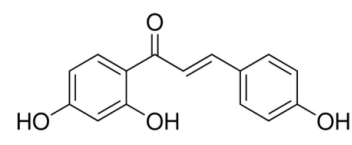

B: Isoliquiritigenin

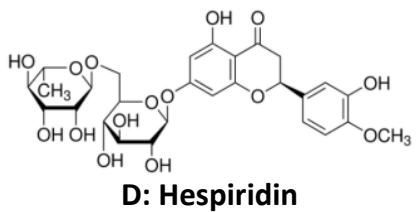

D: Hespiridin

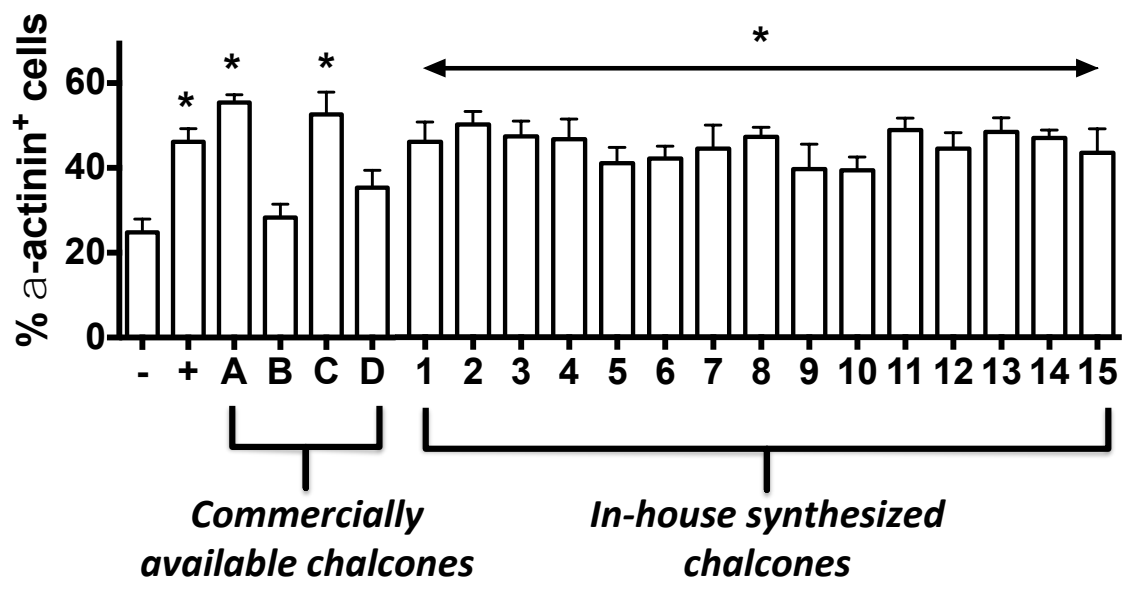

Figure 40: Small scale screen of chalcones as BMP4 mimics during mesoderm induction.

(Top) Chemical structures of compounds 4'-hydroxychalcone (A), isoliquiritigenin (B), cardamonin (C), and hesperidin (D). (Bottom) Flow cytometry analysis of cardiomyocyte ( $\alpha$-actinin) content in monolayer differentiation on culture day 22 ( $n=8-15$ samples/compound). $* P<0.05$ vs. - (negative control; DMSO $0.001 \%$ ) by ANOVA with Dunnett's multiple comparison post-hoc test. -: no BMP4; +: $5 \mathrm{ng} / \mathrm{mL}$ BMP4; compounds 1-15: Refer to Appendix for chemical structures. All compounds were solubilized in DMSO at $10 \mathrm{mmol} / \mathrm{L}$ and tested at $10 \mu \mathrm{mol} / \mathrm{L}$.

\subsubsection{Chalcones do not signal via SMAD or ERK phosphorylation}

BMP4 signals typically via SMAD1/5/8 phosphorylation (Fig. 41A). ERK phosphorylation was not affected by BMP4. Application of 4'-hydroxychalcone or 4-flouro-4'-methoxychalcone (compound 1 chosen randomly from the newly synthesized chalcones) did not phosphorylate SMAD1/5/8 and also did not alter ERK-phosphorylation. Thus, it appears that despite similar outcome with respect to cardiomyocyte differentiation, SMAD signaling may not be involved as mechanism of action associated with BMP4 mimics (Fig. 41). 


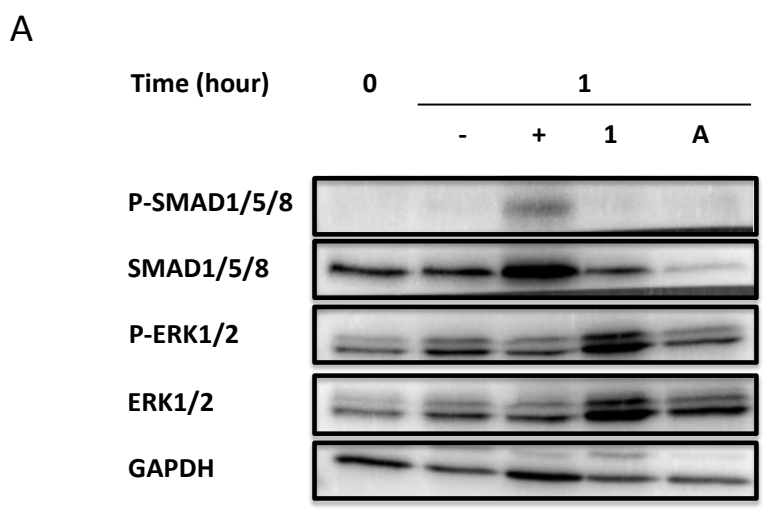

B

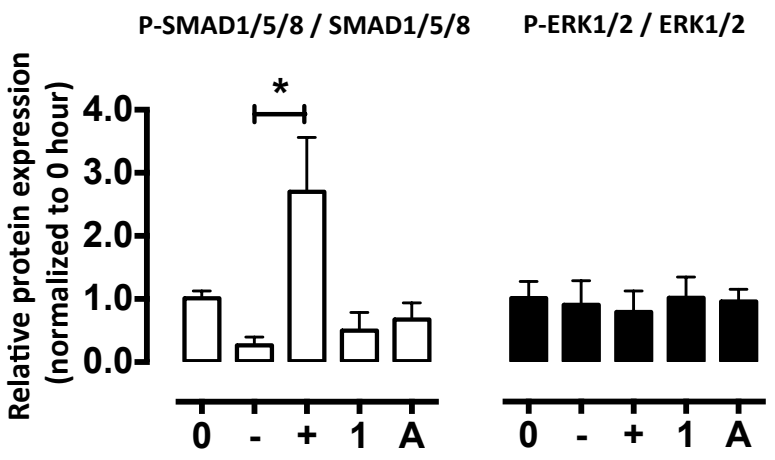

Figure 41: SMAD and ERK phosphorylation in response to BMP4.

HES2 cells were exposed to BMP4 (+; $5 \mathrm{ng} / \mathrm{mL})$, 4-flouro-4'-methoxychalcone (compound 1; $10 \mu \mathrm{mol} / \mathrm{L}$ ) and 4'hydroxychalcone (compound $\mathrm{A} ; 10 \mu \mathrm{mol} / \mathrm{L}$ ) during mesoderm induction in monolayer culture. (A) Western blot analyses of P-SMAD1/5/8, total SMAD1/5/8, P-ERK1/2 and total ERK1/2 in cell lysates at $\mathrm{t}=0$ and 1 hour posttreatment. GAPDH was used as a loading control. (B) Summary of Western blot data showing relative SMAD1/5/8 and ERK1/2 phosphorylation to total SMAD1/5/8 (left) and total ERK1/2 (right; $n=3$ samples/group). $* P<0.05$ vs. $\mathrm{t}=0$ by one-way ANOVA with Dunnett's multiple comparison post-hoc test. 0 : $\mathrm{t}=0$ hour; -: negative control; +: $5 \mathrm{ng} / \mathrm{mL}$ BMP4; 1: 4-flouro-4'-methoxychalcone; A: 4'-hydroxychalcone; SMAD1/5/8: small body size mothers against decapentaplegic 1/5/8; P-SMAD1/5/8: phosphorylated-SMAD1/5/8; ERK1/2: extracellular signal regulated kinase 1/2; P-ERK1/2: phosphorylated-ERK1/2; GAPDH: glyceraldehyde 3-phosphate-dehydrogenase.

\subsubsection{Formation of bioengineered heart muscle with BMP mimics}

Despite several attempts to recapitulate the findings from the monolayer differentiation protocol of BHM culture, it was so far not possible to replace BMP4 by chalcones during mesoderm induction (so far tested: 4'-hydroxychalcone and 4-flouro-4'-methoxychalcone at 10 and 30 $\mu \mathrm{mol} / \mathrm{L}$ ). This is surprising especially in light of the similar effects of BMP4 and the tested 
chalcones in monolayer differentiation. This observation may result from differences in the distribution of chalcones and BMP4 in tissue and will require further investigation and protocol refinement. 


\section{Discussion}

Finding the appropriate model systems to recapitulate body functions and signaling pathways on the molecular, cellular and tissue level have always intrigued researchers. For this reason, several model systems have been developed and employed yet each still presents its own limitations. For instance, animal models have long served their purposes to provide insights on complex human disease mechanisms when human experimentation has not been feasible, but they remain limited by the often fundamental differences in physiology, pathology and pharmacology between human and animal, mostly rodent, models (Shanks et al., 2009).

Cell cultures, and in particular monolayer cultures, reduce the complexity animal models and are thus excellent tools to study very specific molecular signaling events and their implications for cell behavior. However, traditional cell culture models lack the three-dimensionality of bona fide tissue, which creates a highly artificial context with questionable translatability to the relevant in vivo situation (Yin et al., 2016). Finally, the more recently developed 3D culture formats aim at overcoming the context dependent limitation of monolayer cultures, but also have to be considered (bio)artificial, albeit with in many cases closer resemblance to the target organ than classical cell cultures. With the introduction of human pluripotent stem cell models (Takahashi et al., 2007; Thomson et al., 1998), there are now great expectations that differentiation of those cells into defined lineages would advance our tool-set for studying human pathology in the dish.

Our lab, with a focus on myocardial tissue engineering, has introduced several models of engineered heart muscle (EHM), including human EHM from embryonic (Soong et al., 2012; Tiburcy et al., 2014) and induced pluripotent stem cells (Streckfuss-Bomeke et al., 2013). The EHM technology makes use of differentiated cell populations and their culture in a threedimensional collagen type I hydrogel to generate beating heart muscle. More recently, a variation of the initially introduced tissue engineering concept (Langer and Vacanti, 1993) has been introduced, which is termed organoid cultures. Organoids take advantage of the capacity of undifferentiated pluripotent stem cells to develop into differentiated progeny if suspended either at high density with or without a surrounding matrix to support self-assembly into three- 
dimensional tissue-like aggregates (Gjorevski et al., 2014; Lancaster and Knoblich, 2014; Yin et al., 2016). Particular examples for the new organoid approaches include the so called minibrains (Lancaster et al., 2013), liver (Huch et al., 2013; Takebe et al., 2013), kidney (Takasato et al., 2014), prostate (Karthaus et al., 2014). Yet so far no data have been reported for the development of a cardiac organoid.

This thesis builds on previous work by our group on the directed differentiation and selfassembly of mouse and human pluripotent stem cells to form bioengineered heart muscle (BHM) in a single-step organoid approach (Hudson et al., in revision) as a model to simulate human heart development in a dish. The BHM model was inspired by embryonic development, following the hypothesis that hPSCs can assemble BHM if directed to differentiate into predominantly mesodermal cells with a major cardiomyocyte and fibroblast-like cell component in a collagen type I hydrogel to facilitate self-assembly. Developmental stimuli included: (1) Activin-A, BMP4, the GSK-3 inhibitor CHIR99021 for activation of the canonical Wntpathway and FGF-2 (ABCF) for mesoderm induction, (2) the Wnt-inhibitor IWP4 for cardiac specification and (3) TGF $\beta$-1 for cardiac maturation by specifically supporting stroma cell function. Adaptation from 0.4 to $1.2 \mathrm{mmol} / \mathrm{L}$ extracellular calcium during cardiac maturation and ascorbic acid were identified as additional supporting factors. This protocol resulted in robustly contracting BHM after 22 days in culture regardless of the tested human or non-human primate pluripotent stem cell starting population (Hudson et al., in revision).

The main objectives of this thesis were: (1) a thorough characterization of the directed development in BHM, (2) the identification of interventions to further enhance BHM maturation and (3) a replacement of BMP4 with a small molecule BMP4 mimic. The main results of this thesis are summarized below:

- Simulation of human heart development in bioengineered heart muscle

- Evidence for functional neural crest contribution in long-term bioengineered heart muscle culture

- Modeling of faulty cardiogenesis induced by BMP inhibition

- Proof-of-concept for the application of bioengineered heart muscle in drug screening 
- Enhanced functional maturation of bioengineered heart muscle

- Stimulation of mesoderm induction with a small molecule BMP pathway activator

\subsection{Simulation of Human Heart Development in Bioengineered Heart Muscle}

The basic BHM model is comprised of pluripotent stem cells in a collagen type I hydrogel. After a seeding phase ( 24 hour from day -1 to 0 ), mesoderm induction for 3 days is followed by induced cardiac specification for 10 days and cardiac maturation of BHM up to 60 days in this study. The differentiation protocol was adapted from a serum-free monolayer differentiation protocol which took in consideration the synergistic effect of BMP4 and Activin-A in inducing mesoderm from hPSCs (Hudson et al., 2012; Kattman et al., 2011; Lee et al., 2009). Based on previous studies showing the importance of Wnt/ $\beta$-catenin signaling activation for optimal cardiac differentiation (Jackson et al., 2010; Lian et al., 2013; Paige et al., 2010), CHIR99021 was introduced during mesoderm induction phase. Our observation on directed cardiac induction and the importance of proper mesoderm induction are supported by recent studies showing a combinatorial effect of BMP/Activin-A and canonical Wnt signaling (Kim et al., 2015). Several studies have also demonstrated the implication of FGF signaling in the early cardiac induction of stem cells (Burridge et al., 2007; Elliott et al., 2011; Uosaki et al., 2011; Yang et al., 2008; Zhang et al., 2011) which appears to act primarily via ERK/MAPK pathway activation (Aouadi et al., 2006; Wu et al., 2010). Collectively, these observations and our own preliminary studies prompted us to define the ABCF protocol for mesoderm induction.

Studies in the mouse model revealed the importance of $\mathrm{Wnt} / \beta$-catenin deactivation during later stages of embryonic heart development (David et al., 2008; Kwon et al., 2007; Naito et al., 2006). This was further supported by data from in vitro experiments, demonstrating that differentiation of primitive streak cells was enhanced towards cardiac specification upon inhibition of Wnt signaling (Hudson et al., 2012; Karakikes et al., 2014; Yang et al., 2008). Accordingly, we implemented the addition of Wnt-signaling inhibitor IWP4 for cardiac specification, defined as the 10 days following mesoderm induction, in our differentiation protocol. 
Processes underlying cardiomyocyte maturation are not well defined and most likely also include biophysical inputs (Liaw and Zimmermann, 2016). Mechanical stimulation to simulate hemodynamic loading was implemented during cardiac maturation in BHM together with TGF $\beta$-1 stimulation to enhance ECM secretion by fibroblast-like HES progeny. The importance for dynamic mechanical loading for myocardial tissue engineering has been demonstrated previously (Zimmermann et al., 2006). Similarly, evidence for extracellular matrix/fibroblast stabilization by TGF $\beta-1$ stemmed from earlier observations in our lab (Zimmermann et al., 2002). The addition of ascorbic acid followed the rationale that collagen synthesis is dependent on its availability.

RNA-sequencing analyses from BHM culture days -1, 3, 8, 13, 22, 29 and 60 confirmed that BHMs do indeed traverse through defined developmental stages. Mesoderm induction was characterized by the loss of the pluripotent marker OCT4 (Hough et al., 2009; Laslett et al., 2007) and transient expression of early mesodermal markers such as MIXL1 (Tam and Loebel, 2007), which in turn induces the expression of the master regulator of cardiovascular lineage specification MESP1 (Bondue et al., 2008; Bondue et al., 2011; David et al., 2008; Paige et al., 2015). The transient expression of MESP1, peaking at day 3 in the BHM, appeared to be involved in the commitment of the mesodermally induced cells towards the cardiomyocyte lineage; this notion was supported by the expression of early cardiac progenitor markers including NKX2-5 and ISL1 (peak at day 8) and a concurrent increase in the expression of TBX5 and ACTC1 (Behrens et al., 2013; Cai et al., 2003; Dierickx et al., 2012; Elliott et al., 2011; Moretti et al., 2006; Wu et al., 2006). The cardiac maturation phase was finally marked with the upregulation of late cardiac markers MYH7 and MYL2 (Beqqali et al., 2006). Taken together, time course gene expression profiling confirmed also on the molecular level that BHMs recapitulate defined stages of cardiac development.

Enzymatic dissociation of BHMs at day 22 and subsequent analyses of cell composition using flow cytometry showed that our BHM differentiation protocol, unlike previous methodologies, did not involve directed differentiation of pluripotent stem cells to cardiomyocytes only (Burridge et al., 2015; Burridge et al., 2014; Burridge et al., 2011; Karakikes et al., 2014; Lian et al., 2015; Lian et al., 2013; Mummery et al., 2012), but also parallel development of stroma cells from the same starting cell source. Both cell types are required for proper myocardial tissue 
formation (Kensah et al., 2012; Naito et al., 2006; Nunes et al., 2013; Tiburcy et al., 2011; Zhang et al., 2013). Furthermore, traces of hematopoietic cells (marked by CD117 and CD45) and endothelial cells (marked by CD31) were observed, which may have originated from mesodermal cell population expressing $T$ and MIXL1 (Davis et al., 2008; Kennedy et al., 2007; Lim et al., 2013; Murry and Keller, 2008). It is important to note that the inhibition of Wnt signaling with IWP4 starting day 3 (the peak of mesodermal marker expression) was introduced to favor cardiac mesoderm formation over hematopoietic and vascular differentiation (Lian et al., 2014; Murry and Keller, 2008; Nostro et al., 2008; Zhu et al., 2013).

Knowing that myocardium, epicardium and endocardium are derived from a common precursor (Misfeldt et al., 2009; Witty et al., 2014), additional gene expression and morphological analyses were employed to screen for potential lineage diversification. Indeed, differential expression of CD31 and VE-Cadherin as well as WTI and ALDH1A2 during BHM development suggested distinct endocardial and epicardial activity. Their contribution to the BHM formation process will need further investigations.

BHMs started to beat by culture day 13 with continuous development into a highly synchronized contractile activity until culture day 22. Isometric force measurements revealed that BHMs displayed organotypic responses to preloading (Frank-Starling) and positive inotropy in response to increasing extracellular calcium concentrations and isoprenaline for $\beta$-adrenergic stimulation (Hirt et al., 2014; Schaaf et al., 2011; Soong et al., 2012; Streckfuss-Bomeke et al., 2013; Tulloch et al., 2011). Impaling electrode measurements identified mostly ventricular working myocardium like cells in BHM at culture day 22. The generation of a mixture of cardiac cell types has been demonstrated previously (Hudson et al., 2012; Kehat et al., 2001; Mummery et al., 2003; Norstrom et al., 2006; Yoon et al., 2006). Note, however, that BHMs at day 22 possess immature action potential parameters as opposed to an adult ventricular cardiomyocyte (AVCM); such as automaticity, slower upstroke velocity (13 V/s for BHM vs. $300 \mathrm{~V} / \mathrm{s}$ for AVCM), higher membrane potential ( $-42 \mathrm{mV}$ for BHM vs $-85 \mathrm{mV}$ for AVCM) and lower action potential amplitude ( $64 \mathrm{mV}$ for BHM vs. $110 \mathrm{mV}$ for AVCM). Yet the parameters recorded in BHM are higher than those recorded for fetal-like ventricular cardiomyocytes (He et al., 2003; Mummery et al., 2003; Rajala et al., 2011; Schram et al., 2002). Importantly, there was an upregulation of relevant cardiac ion channels (SCN5a, HCN4, CACNAlC and KCNH2) 
and cardiac calcium homeostasis associated genes (RYR2, ATP2A2, PLN and CASQ2) by culture day 22 suggesting maturation of the electromechanical machinery in cardiomyocytes. Currently refined differentiation methods may allow for directed differentiation also into predominantly atrial and pacemaker-like cell rich BHMs or even BHM composed of defined cell mixtures (Birket et al., 2015; Devalla et al., 2015; Schwach and Passier, 2016).

In line with the concept of continuous cardiomyocyte maturation, we observed a significant increase in maximal inotropic capacity and rightwards shift in calcium $\mathrm{EC}_{50}$ as well as responsiveness to $\beta$-adrenergic stimulation with concurrently enhanced network formation, comprised of elongated and striated cardiomyocytes. Collectively, we interpret the available data as evidence for enhanced cardiomyocyte maturation in BHM as compared to the maturation observed in extended monolayer cultures (Kamakura et al., 2013; Lundy et al., 2013).

It is important to acknowledge that BHMs in the present format present lower absolute force as compared to the classical tissue engineering approach, using differentiated cardiomyocytes supported by typically collagen or fibrin matrix (Hirt et al., 2014; Kensah et al., 2012; Nunes et al., 2013; Schaaf et al., 2011; Soong et al., 2012; Streckfuss-Bomeke et al., 2013; Tiburcy et al., 2011; Tulloch et al., 2011; Zhang et al., 2013). Also structurally, cardiomyocytes in BHM appear to be less mature as compared to cardiomyocytes in our classical engineered heart muscle (EHM) model (Soong et al., 2012; Tiburcy et al., 2014), which is developed from cell mixtures of different developmental stage (cardiomyocyte from 22 day monolayer differentiation as outlined here with fibroblasts from either foreskin [early postnatal] or skin from advanced aged donors). Despite this apparent technological shortcoming, BHM represent to our knowledge the only model, allowing for a recapitulation of embryonic to fetal human heart development from a homogenous pluripotent stem cell source. This may have particular advantages for example in the modeling and identification of embryonic heart toxicity, congenital heart disease and early potentially clinically not observable dysfunction in hereditary cardiomyopathies. Finally, also as an experimental tool for studies of physiological processes of heart development and maturation, BHM appear to be applicable. 


\subsection{Evidence for Functional Neuro-crest Contribution in Bioengineered Heart Muscle}

A surprising observation was the occurrence of a strong ectodermal molecular finger-print in 60 day BHM cultures. This coincided with the development of variable cyst-like structures at the BHM surface. Force of contraction and BHM inner structure was not obviously affected by these morphological perturbations. Bioinformatics analyses revealed a strong activation of an ectodermal transcription program in day $60 \mathrm{BHM}$ pointing to neural crest development. The neural crest contributes the sympathetic nerve system to the heart and is thought to invade the heart along vascular structures (Buitrago-Delgado et al., 2015; Donoghue et al., 2008; Hall and Gillis, 2013). BHM do not contain a vascular component, but exhibit neurosphere like structures only in day 60 cultures. These neurospheres co-stained with tyrosine hydroxylase (TH), being the rate limiting enzyme in catecholamine production in the sympathetic nerve system (Daubner et al., 2011; Molinoff and Axelrod, 1971).

To test for the functional relevance of catecholamine production and storage from sympathetic vesicles, reserpine was applied chronically (for two weeks) and acutely in long term BHM culture. While chronic treatment, did not impart a notable effect on BHM development and basal function, there was evidence for a negative chronotropic effect by reserpine mediated blockade of VMAT as anticipated (Mandela et al., 2010; Metzger et al., 2002; Yamamoto et al., 2007) likely as a consequence of depletion of catecholamine storage vesicles present only in the chronically conditioned BHM. Untreated BHM showed a trend towards a stable increase in beating rate which may be mediated by unspecific reserpine action on cholinergic stimulation or alternatively could suggest a constant catecholamine leak from less matured sympathetic neurons. This needs to be further investigated to elucidate the relevance of the "heart and brain" interaction in BHM.

Neuroectoderm differentiation from hPSCs has been shown to be inhibited by BMP, Wnt and Activin signaling (Aubert et al., 2002; Murry and Keller, 2008; Ying et al., 2003). Detailed transcriptome analyses confirmed that there were already hints for ectoderm activation after culture day 8 (after mesoderm induction). Thus, it may be concluded that inhibition of neuroectoderm differentiation was insufficient during mesoderm induction. However, it is 
notable that the surge in ectoderm expression with a strong neuro crest component occurred during a time window similar to in utero sympathetic nerve development (McCulley and Black, 2012). Despite the evidence for cardiac mesoderm formation primarily in our BHM differentiation, there was minimal ectodermal contamination by day 22 marked by the presence of NEUROD1 expressing cells. Here we hypothesize that neural crest precursors remained dormant when most cells were mesodermally differentiated with an activation in long-term BHM culture (Buitrago-Delgado et al., 2015; Donoghue et al., 2008; Hall and Gillis, 2013). Considering the role of TGF $\beta-1$ in regulation of neural crest cell proliferation (Chai et al., 2003; Sasaki et al., 2006), $1 \mathrm{ng} / \mathrm{mL}$ of TGF $\beta-1$ supplemented in our prolonged BHM culture may have promoted neural development marked by elevated expression of PAX3 (Donoghue et al., 2008), SOX5 (Buitrago-Delgado et al., 2015) and more importantly a neuroectodermal specific marker SOX1 (Ying et al., 2003) by BHM culture day 60 (RNA sequencing data).

These parallel interdependent processes of cardiac maturation and cardiac neurogenesis imply that BHMs follow a complex in utero-like development with a cardiac-neuro crosstalk. This needs to be further explored to dissect the role of neural crest cells in the functional maturation of cardiomyocytes not only in BHMs, but also in fetal heart development.

\subsection{Modeling of Faulty Cardiogenesis Induced by BMP Inhibition}

In an attempt to test the utility of BHMs in disease modeling, we sought to validate whether faulty cardiogenesis upon BMP inhibition can be induced in our BHM model. Studies in mouse and chick demonstrated the importance of BMP signaling in cardiac cushion formation (Kruithof et al., 2012) and myocardial differentiation of cardiac progenitors beyond mesoderm induction phase (Wang et al., 2010). Furthermore in collaboration with Dr. Zeidler and Prof. E. Wingender (Institute of Bioinformatics, University Medical Center Goettingen), we were able to collect evidence for enhanced SMAD signaling and inhibition of canonical Wnt signaling during cardiac specification of BHM development (Zeidler et al., 2016).

Accordingly, the application of BMP inhibitors including dorsomorphin (Hao et al., 2008; Yu et al., 2008) and the highly selective DMH1 (Hao et al., 2010) during cardiac specification (from 
BHM culture day 6) resulted in a block in cardiomyogenesis with subsequent contractile failure of BHM. Similar studies cannot be performed well in animal models, because a complete knock-out of BMP-receptors turned out to be embryonically lethal and conditional knock-outs tended to be confounded by hemodynamic disturbances likely due to malformation of the cardiac cushion and related heart valves (Gaussin et al., 2002; Kruithof et al., 2012; Yang et al., 2006).

Thus, we interpret our data as first evidence for a specific supportive effect of BMP-signaling at the cardiac progenitor cell stage for heart muscle formation. Note that BMP4 was only supplemented during mesoderm induction to stabilize endogenous BMP4 levels. BMP4 expression peaks at day 8 of BHM development (RNAseq data - not shown) and thus inhibition of BMP-signaling with dorsomorphin or DMH1 starting from day 6 of BHM culture should have been maximally effective. In addition to the inhibition of the canonical BMP4 receptors ACVR1 (ALK2), BMPR1A (ALK3) and BMPR1B (ALK6) dorsomorphin also inhibits AMPK $\left(\mathrm{K}_{\mathrm{i}}=109 \mathrm{nmol} / \mathrm{L}\right)$ and other signal pathways. The similar effect of the highly BMPR-selective inhibitor DMH1 and dorsomorphin suggests that indeed BMP-signaling is key for proper heart muscle specification and maturation in BHM.

\subsection{Proof-of-concept for the Application of BHM in Drug Screening}

Due to the finding that transcription of cardiac ion channel and calcium handling protein encoding genes and calcium sensitivity were more advanced towards the anticipated adult phenotype in culture day 29 BHM, we chose to investigate the biological activity of classical reference compounds (digitoxin, verapamil, E4031) with a focus on inotropy, lusitropy, and chronotropy in BHM of this stage and found that all investigated drugs caused the anticipated phenotype. This is in short: (1) for digitoxin an increase in contractile force; (2) for verapamil a decrease in contractile force and shortening of the action potentials with a concurrent increase in beating frequency; (3) for E4031 no effect on contractile force, but a prolongation of relaxation. The latter was associated with an increase in action potential duration (He et al., 2003). These data collectively suggest that BHM, despite its immaturity, can be used to identify the biological activity and drug associated risk, for example of HERG channel blockers (E4031). These studies 
need to be extended to further scrutinize and compare the responsiveness of BHM to responses observed in bona fide human myocardium.

\subsection{Enhanced Functional Maturation of Bioengineered Heart Muscle}

We next postulated that BHM function can be further enhanced by optimizing the existing differentiation protocol by (1) re-evaluating the optimal TGF $\beta$-1 stimulus (concentration and timing) for BHM formation, (2) omitting insulin stimulation, and (3) changing the basal medium from RPMI to Iscove's medium especially for cardiac maturation in long term BHM culture.

TGF $\beta$-1 has been found to improve cardiomyogenic differentiation of hPSCs and cardiac progenitor cells (Goumans et al., 2008; Li et al., 2005). Yet, being a multifunctional growth factor, TGF $\beta-1$ also stimulates synthesis of extracellular matrix components in stromal cells (Gooch et al., 2004; Muller-Pillasch et al., 1997; Nakamura et al., 1992; Sugiyama et al., 2013). This suggests a possible role of TGF $\beta-1$ on both the cardiomyocyte and stromal cell population in BHMs. Here we defined that TGF $\beta-1$ effectively supports BHM formation if added during culture days 13-22 at (1-10 ng/mL). Interestingly, long term stimulation for an additional week (until day 29) or an additional month (until day 60) resulted in inferior outcome with reduced contractile performance. A stronger compaction of BHM (reflected by a decrease in cross sectional area) under long term TGF $\beta-1(10 \mathrm{ng} / \mathrm{mL})$ suggests enhanced fibroblast functionality and potentially fibroblast to myofibroblast transformation. While the latter is interesting for example for disease modeling, additional studies would be needed to define the underlying mechanisms of the functional and structural deterioration.

Insulin and insulin-like growth factor (IGF) signaling activate and mediate prosurvival and hypertrophic growth effects via the protein kinase B (Akt/PKB) pathway (Freund et al., 2008). Insulin was shown to on the one hand direct early cardiomyogenesis in mouse (McDevitt et al., 2005; Naito et al., 2005) and on the other hand support EHM formation (Naito et al., 2006). Other groups reported that insulin inhibits early cardiomyogenesis (Freund et al., 2008; Lian et al., 2013; Lian et al., 2013; Xu et al., 2008). In a recent study (Lian et al., 2013), insulin indeed inhibited hPSC cardiac differentiation. The authors continue to demonstrate that this effect can 
be rescued in the presence of Wnt signaling modulators as CHIR99021 and IWP4. The inhibitory effect of insulin signaling reported above may have seem overcome during early BHM development by the presence of Wnt pathway modulators. Here we tested whether insulin would be supportive or not during later phases of BHM differentiation and found that insulin is not required during cardiac maturation, i.e., from culture day 13 onwards. This appears to contrast previous studies showing the positive effects of insulin signaling on the proliferation of differentiated cardiomyocytes (Li et al., 2011; McDevitt et al., 2005; Xin et al., 2011). In BHM we could not find any evidence for enhanced cell proliferation under insulin stimulation. Whether insulin or IGF-1 via AKT mediated pro-survival signaling would be protective under "pathological" stimuli remains to be tested.

In an attempt to reduce the precipitation of insoluble calcium phosphate in long-term cultures of RPMI treated BHMs, we investigated the use of Iscove's medium during late stages of BHM maturation (from BHM culture day 29 upto day 60). Indeed the presence of suitable calcium levels $(1.2 \mathrm{mmol} / \mathrm{L})$ and lower phosphate content in Iscove's medium resulted in enhaned contractile performance and increased total cell count in particular the cardiomyocyte content.

Based on the above data, we now propose a refined long-term BHM cultivation protocol as depicted in the following schematic (Fig. 42). 


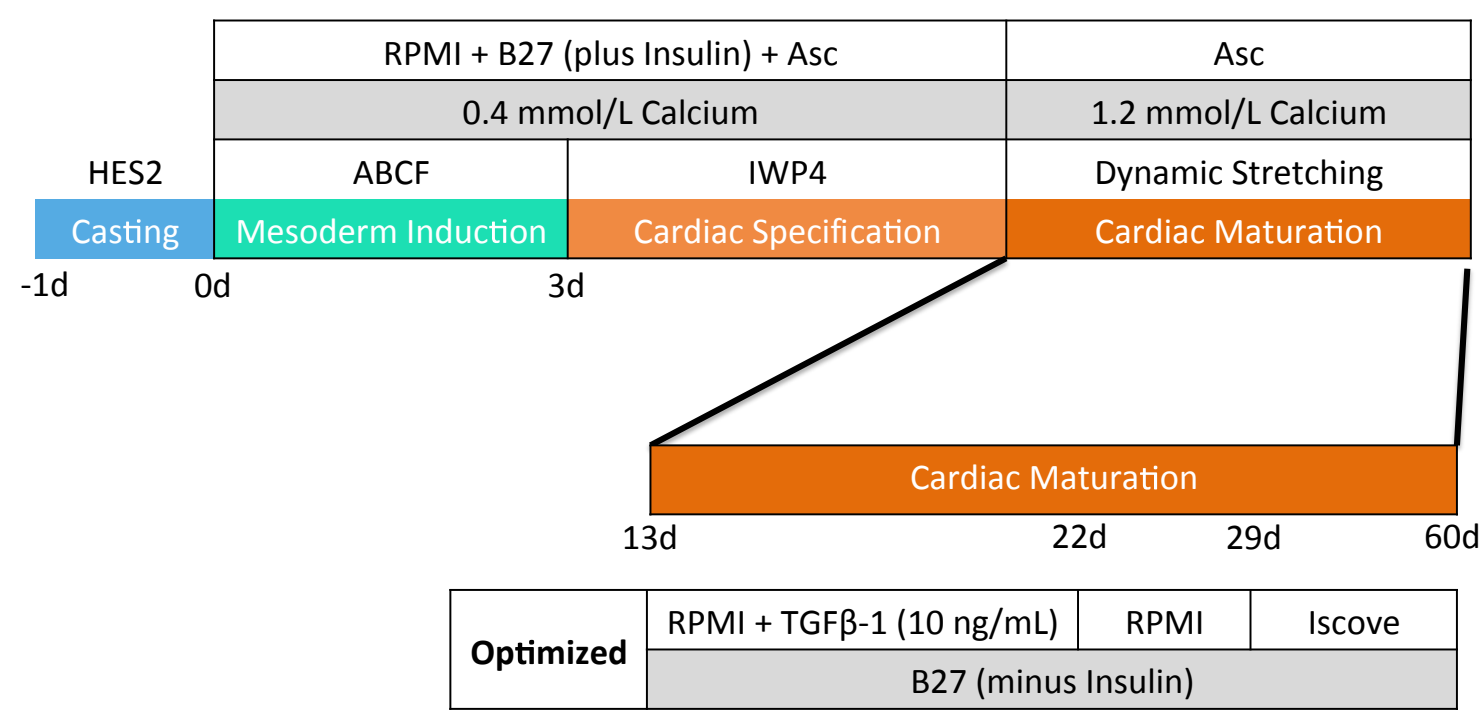

Figure 42: Schematic outline of the optimized long-term BHM culture/maturation protocol.

\subsection{Stimulation of Mesoderm Induction with a Small Molecule BMP Mimic}

During embryogenesis, many signaling pathways work simultaneously which can have a positive feedback on one another at one point and conflict each other at other time points. For this reason, understanding the underlying mechanisms of these pathways are the key steps to targeting them for a more precise control over stem-cell differentiation into a particular lineage. Four major pathways are implicated in the early induction of mesoderm including TGF $\beta /$ Activin/Nodal, BMP, WNT and FGF (Burridge et al., 2007; Elliott et al., 2011; Hudson et al., 2012; Jackson et al., 2010; Kattman et al., 2011; Kim et al., 2015; Paige et al., 2010; Yang et al., 2008; Zhang et al., 2011). Several groups have reported that introducing BMPs at specific time points is crucial for the induction of cardiac mesoderm (Burridge et al., 2011; Hudson et al., 2012; Laflamme et al., 2007; Yang et al., 2008; Yao et al., 2006). Yet BMP is a recombinant protein which is both expensive and has a significant batch-to-batch variability in its biological activity (Peterson and Loring, 2012). For this reason, we postulated that small molecule replacements of BMP4 will enable a better control in monolayer and BHM cultures. 
Prior to the screening for BMP4 mimics, we re-evaluated the temporal and concentration dependent effects of BMP4 on mesoderm induction and ultimately cardiomyocyte differentiation in a monolayer culture format. We first used the previously established concentration of $5 \mathrm{ng} / \mathrm{mL}$ BMP4 (Hudson et al., in revision) and varied the duration of stimulation from the first 24 to 48 and finally 72 hour of mesoderm induction. These experiments confirmed 48-72 hour as an optimal window for BMP4 stimulation. As to cardiomyocyte and concurrent fibroblast differentiation, $5 \mathrm{ng} / \mathrm{mL}$ were confirmed as optimally inductive, resulting in cell populations comprised of $\sim 50 \%$ cardiomyocytes and $\sim 20 \% \mathrm{CD}^{+}$ fibroblast-like cells. Importantly, higher BMP4 concentrations resulted in markedly decreased cardiomyocyte and increase fibroblast content. Accordingly, BHM formation was optimally supported by 5-10 ng/mL BMP4. The slightly higher "tolerated" BMP4 concentrations (10 $\mathrm{ng} / \mathrm{mL}$ ) in BHM may hint towards differences in monolayer vs. tissue availability of BMP4.

The presence of Activin A which acts as a surrogate for NODAL and the Wnt agonist CHIR99021 in our culture format was enough to induce loss of pluripotency but not cardiac mesoderm induction evidenced by no increase in MESP1 in the absence of BMP4 during the designated mesoderm induction phase. Consequently, identification of the precise differentiation events would require further transcriptome studies for example to identify early mesodermal marker $T$ and FLK1 for paraxial mesoderm which could give rise to hemopoetic and endothelial cell lineage as well as defintive endoderm in the absence of exogenous BMP4 (Martin-Puig et al., 2008; Murry and Keller, 2008; Orlova et al., 2016; Tam and Loebel, 2007). Moreover the induction of ectodermal cell population should be studied further by screens for NEUROD1, PAX6 and SOX1 expression (Aubert et al., 2002; Murry and Keller, 2008; Noisa et al., 2012; Ying et al., 2003).

Finally, the available transcriptome data (MESP1 and ISL1 induction, but no NKX2-5 induction) could argue for development of extraembryonic mesoderm (Arnold and Robertson, 2009; Orlova et al., 2016) under high BMP4 concentrations (30 ng/mL). Collectively, this data confirm the important role of BMP4 for cardiac mesoderm induction in monolayer and BHM formats. This effect was time and concentration dependent with $5 \mathrm{ng} / \mathrm{mL}$ BMP4 added for 3 days during the full mesoderm induction phase being identified as optimal for the induction of 
cardiomyocyte and stromal cell differentiation. Both cell components are crucial for the formation of tissue engineered myocardium (Naito et al., 2006).

The compelling evidence for the central role of BMP4 for mesoderm induction and subsequent cardiomyogenesis in vitro and in vivo (Burridge et al., 2015; Burridge et al., 2014; Hao et al., 2008; Hao et al., 2010; Hudson et al., 2012; Karakikes et al., 2014; Lian et al., 2014; Lian et al., 2015; Lian et al., 2013; Wang et al., 2011; Willems et al., 2009; Willems et al., 2011; Zhang et al., 2011) prompted us for the search for small molecule BMP4 signal pathway activators. A recent study, using a SMAD-response element to drive the expression of the luciferase gene, identified chalcones (Vrijens et al., 2013) as potential BMP signal stimulators.

We tested 4 commercially available and 15 newly synthesized chalcones as BMP4 replacements during mesoderm induction first in monolayer culture. This identified 4'-hydroxychalcone and 4-flouro-4'-methoxychalcone as equally effective at $10 \mu \mathrm{mol} / \mathrm{L}$ in the induction of cardiomyogenesis as BMP4 $(5 \mathrm{ng} / \mathrm{mL})$. Interestingly, both compounds apparently did not phosphorylate and thus activate SMAD1/5/8. Thus we concluded a differential so far undefined mechanism of action for the induction of BMP4 signaling in HES during mesoderm induction.

The ultimate goal of a more defined BHM using BMP4 replacements could however not be reached since the tested chalcones (4'-hydroxychalcone and 4-fluoro-4'-methoxychalcone) failed to induce cardiac differentiation during BHM development even at higher concentrations (30 $\mu \mathrm{mol} / \mathrm{L})$. This may be attributed to the diffusion limits in the BHM as compared to monolayer cultures, stability of the compound within the collagen hydrogel and hydrophilic/phobic properties of the compounds (El-Sayed and Gaber, 2015; Simmler et al., 2013). We will attempt to further modify the chemical structure of the identified hit molecules and continue to search for alternative replacements of BMP4 with better tissue permeability. 


\section{Conclusion and Outlook}

In this thesis, the first detailed characterization of a novel cardiac organoid model, namely of bioengineered heart muscle (BHM), at the morphological, functional and molecular level is provided. A key observation was that BHM traverse through defined developmental stages and thus recapitulate human cardiomyogenesis from the embryonic to an early fetal stage. This allowed for the investigation of proper and faulty cardiomyogenesis as well as the responsiveness to classical pharmacological interventions targeting the heart.

Despite not having reached adult-like maturity, we anticipate that BHM will be an attractive tool in mechanistic studies of congenital heart disease and hereditary cardiomyopathies. The guided differentiation into a multicellular mesodermal tissue with neuro-crest contribution at later stages of differentiation was an unanticipated observation, suggesting that advanced maturation may depend crucially on sympathetic inputs. Future studies will be directed to dissect the interplay of mesoderm and ectoderm in heart development and maturation. This will also include the further definition of "guiding" signaling pathways and the replacement of all growth factors by well-defined small molecules. The latter may finally also be of therapeutic relevance in heart disease. 


\section{Bibliography}

Adewumi O AB, Ahrlund-Richter L, Amit M, Andrews PW (2007) Characterization of human embryonic stem cell lines by the International Stem Cell Initiative. Nat Biotech. 25: 803816.

Allegrucci C, Young LE (2007) Differences between human embryonic stem cell lines. Hum Reprod Update. 13: 103-120.

Aouadi M, Bost F, Caron L, Laurent K, Le Marchand Brustel Y, Binétruy B (2006) p38 Mitogen-Activated Protein Kinase Activity Commits Embryonic Stem Cells to Either Neurogenesis or Cardiomyogenesis. Stem Cells. 24: 1399-1406.

Arnold SJ, Robertson EJ (2009) Making a commitment: cell lineage allocation and axis patterning in the early mouse embryo. Nat Rev Mol Cell Biol. 10: 91-103.

Aubert J, Dunstan H, Chambers I, Smith A (2002) Functional gene screening in embryonic stem cells implicates Wnt antagonism in neural differentiation. Nat Biotechnol. 20: 12401245.

Babitt JL, Zhang Y, Samad TA, Xia Y, Tang J, Campagna JA, Schneyer AL, Woolf CJ, Lin HY (2005) Repulsive Guidance Molecule (RGMa), a DRAGON Homologue, Is a Bone Morphogenetic Protein Co-receptor. Journal of Biological Chemistry. 280: 2982029827.

Batovska DI, Todorova IT (2010) Trends in utilization of the pharmacological potential of chalcones. Curr Clin Pharmacol. 5: 1-29.

Behrens AN, Iacovino M, Lohr JL, Ren Y, Zierold C, Harvey RP, Kyba M, Garry DJ, Martin CM (2013) Nkx2-5 Mediates Differential Cardiac Differentiation Through Interaction with Hoxa10. Stem Cells Dev. 22: 2211-2220.

Beqqali A, Kloots J, Ward-van Oostwaard D, Mummery C, Passier R (2006) Genome-wide transcriptional profiling of human embryonic stem cells differentiating to cardiomyocytes. Stem Cells. 24: 1956-1967.

Binah O, Dolnikov K, Sadan O, Shilkrut M, Zeevi-Levin N, Amit M, Danon A, Itskovitz-Eldor J (2007) Functional and developmental properties of human embryonic stem cellsderived cardiomyocytes. J Electrocardiol. 40: S192-196.

Birket MJ, Ribeiro MC, Verkerk AO, Ward D, Leitoguinho AR, den Hartogh SC, Orlova VV, Devalla HD, Schwach V, Bellin M, Passier R, Mummery CL (2015) Expansion and patterning of cardiovascular progenitors derived from human pluripotent stem cells. Nat Biotechnol. 33: 970-979.

Bondue A, Lapouge G, Paulissen C, Semeraro C, Iacovino M, Kyba M, Blanpain C (2008) Mesp1 acts as a master regulator of multipotent cardiovascular progenitor specification. Cell Stem Cell. 3: 69-84. 
Bondue A, Tannler S, Chiapparo G, Chabab S, Ramialison M, Paulissen C, Beck B, Harvey R, Blanpain C (2011) Defining the earliest step of cardiovascular progenitor specification during embryonic stem cell differentiation. J Cell Biol. 192: 751-765.

Brito-Martins M, Harding SE, Ali NN (2008) beta(1)- and beta(2)-adrenoceptor responses in cardiomyocytes derived from human embryonic stem cells: comparison with failing and non-failing adult human heart. Br J Pharmacol. 153: 751-759.

Bruneau BG, Logan M, Davis N, Levi T, Tabin CJ, Seidman JG, Seidman CE (1999) Chamberspecific cardiac expression of Tbx5 and heart defects in Holt-Oram syndrome. Dev Biol. 211: 100-108.

Buitrago-Delgado E, Nordin K, Rao A, Geary L, LaBonne C (2015) NEURODEVELOPMENT. Shared regulatory programs suggest retention of blastula-stage potential in neural crest cells. Science. 348: 1332-1335.

Burridge PW, Anderson D, Priddle H, Barbadillo Munoz MD, Chamberlain S, Allegrucci C, Young LE, Denning C (2007) Improved human embryonic stem cell embryoid body homogeneity and cardiomyocyte differentiation from a novel V-96 plate aggregation system highlights interline variability. Stem Cells. 25: 929-938.

Burridge PW, Holmstrom A, Wu JC (2015) Chemically Defined Culture and Cardiomyocyte Differentiation of Human Pluripotent Stem Cells. Curr Protoc Hum Genet. 87: 21.23.2115.

Burridge PW, Keller G, Gold JD, Wu JC (2012) Production of De Novo Cardiomyocytes: Human Pluripotent Stem Cell Differentiation and Direct Reprogramming. Cell Stem Cell. 10: 16-28.

Burridge PW, Matsa E, Shukla P, Lin ZC, Churko JM, Ebert AD, Lan F, Diecke S, Huber B, Mordwinkin NM, Plews JR, Abilez OJ (2014) Chemically defined generation of human cardiomyocytes. 11: 855-860.

Burridge PW, Thompson S, Millrod MA, Weinberg S, Yuan X, Peters A, Mahairaki V, Koliatsos VE, Tung L, Zambidis ET (2011) A Universal System for Highly Efficient Cardiac Differentiation of Human Induced Pluripotent Stem Cells That Eliminates Interline Variability. PLoS One. 6: e18293.

Cagavi E, Bartulos O, Suh CY, Sun B, Yue Z, Jiang Z, Yue L, Qyang Y (2014) Functional Cardiomyocytes Derived from Isl1 Cardiac Progenitors via Bmp4 Stimulation. PLoS One. 9.

Cai CL, Liang X, Shi Y, Chu PH, Pfaff SL, Chen J, Evans S (2003) Isl1 identifies a cardiac progenitor population that proliferates prior to differentiation and contributes a majority of cells to the heart. Dev Cell. 5: 877-889.

Carrier RL, Papadaki M, Rupnick M, Schoen FJ, Bursac N, Langer R, Freed LE, VunjakNovakovic G (1999) Cardiac tissue engineering: cell seeding, cultivation parameters, and tissue construct characterization. Biotechnol Bioeng. 64: 580-589. 
Chai Y, Ito Y, Han J (2003) TGF-beta signaling and its functional significance in regulating the fate of cranial neural crest cells. Crit Rev Oral Biol Med. 14: 78-88.

Chan MC, Nguyen PH, Davis BN, Ohoka N, Hayashi H, Du K, Lagna G, Hata A (2007) A novel regulatory mechanism of the bone morphogenetic protein (BMP) signaling pathway involving the carboxyl-terminal tail domain of BMP type II receptor. Mol Cell Biol. 27: 5776-5789.

Chang H, Zwijsen A, Vogel H, Huylebroeck D, Matzuk MM (2000) Smad5 is essential for leftright asymmetry in mice. Dev Biol. 219: 71-78.

Chen H, Shi S, Acosta L, Li W, Lu J, Bao S, Chen Z, Yang Z, Schneider MD, Chien KR, Conway SJ, Yoder MC, Haneline LS, Franco D, Shou W (2004) BMP10 is essential for maintaining cardiac growth during murine cardiogenesis. Development. 131: 2219-2231.

Corradini E, Babitt JL, Lin HY (2009) The RGM/DRAGON family of BMP co-receptors. Cytokine Growth Factor Rev. 20: 389-398.

Costello I, Pimeisl IM, Drager S, Bikoff EK, Robertson EJ, Arnold SJ (2011) The T-box transcription factor Eomesodermin acts upstream of Mesp1 to specify cardiac mesoderm during mouse gastrulation. Nat Cell Biol. 13: 1084-1091.

Daubner SC, Le T, Wang S (2011) Tyrosine Hydroxylase and Regulation of Dopamine Synthesis. Archives of biochemistry and biophysics. 508: 1-12.

David R, Brenner C, Stieber J, Schwarz F, Brunner S, Vollmer M, Mentele E, Müller-Höcker J, Kitajima S, Lickert H, Rupp R, Franz WM (2008) MesP1 drives vertebrate cardiovascular differentiation through Dkk-1-mediated blockade of Wnt-signalling. Nat Cell Biol. 10: 338-345.

Davis RP, Ng ES, Costa M, Mossman AK, Sourris K, Elefanty AG, Stanley EG (2008) Targeting a GFP reporter gene to the MIXL1 locus of human embryonic stem cells identifies human primitive streak-like cells and enables isolation of primitive hematopoietic precursors. Blood. 111: 1876-1884.

Devalla HD, Schwach V, Ford JW, Milnes JT, El-Haou S, Jackson C, Gkatzis K, Elliott DA, Chuva de Sousa Lopes SM, Mummery CL, Verkerk AO, Passier R (2015) Atrial-like cardiomyocytes from human pluripotent stem cells are a robust preclinical model for assessing atrial-selective pharmacology. EMBO Mol Med. 7: 394-410.

Didié M, Christalla P, Rubart M, Muppala V, Döker S, Unsöld B, El-Armouche A, Rau T, Eschenhagen T, Schwoerer AP, Ehmke H, Schumacher U, Fuchs S, Lange C, Becker A, Tao W, Scherschel JA, Soonpaa MH, Yang T, Lin Q, Zenke M, Han D, Schöler HR, Rudolph C, Steinemann D, Schlegelberger B, Kattman S, Witty A, Keller G, Field LJ, Zimmermann WH (2013) Parthenogenetic stem cells for tissue-engineered heart repair. The Journal of Clinical Investigation. 123: 1285-1298. 
Dierickx P, Doevendans PA, Geijsen N, van Laake LW (2012) Embryonic template-based generation and purification of pluripotent stem cell-derived cardiomyocytes for heart repair. Journal of cardiovascular translational research. 5: 566-580.

Dijke P, Heldin C, Miyazono K, Maeda S, Imamura T. (2006). Smad Transcriptional CoActivators and Co-Repressors Smad Signal Transduction (Vol. 5, pp. 277-293): Springer Netherlands.

Dolnikov K, Shilkrut M, Zeevi-Levin N, Danon AA, Gerecht-Nir S, Itskovitz-Eldor J, Binah O (2005) Functional Properties of Human Embryonic Stem Cell-Derived Cardiomyocytes. Annals of the New York Academy of Sciences. 1047: 66-75.

Donoghue PCJ, Graham A, Kelsh RN (2008) The origin and evolution of the neural crest. BioEssays : news and reviews in molecular, cellular and developmental biology. 30: 530-541.

Dopazo J, Carazo JM (1997) Phylogenetic reconstruction using an unsupervised growing neural network that adopts the topology of a phylogenetic tree. J Mol Evol. 44: 226-233.

El-Sayed YS, Gaber M (2015) Studies on chalcone derivatives: complex formation, thermal behavior, stability constant and antioxidant activity. Spectrochim Acta A Mol Biomol Spectrosc. 137: 423-431.

Elliott DA, Braam SR, Koutsis K, Ng ES, Jenny R, Lagerqvist EL, Biben C, Hatzistavrou T, Hirst CE, Yu QC, Skelton RJ, Ward-van Oostwaard D, Lim SM, Khammy O, Li X, Hawes SM, Davis RP, Goulburn AL, Passier R, Prall OW, Haynes JM, Pouton CW, Kaye DM, Mummery CL, Elefanty AG, Stanley EG (2011) NKX2-5(eGFP/w) hESCs for isolation of human cardiac progenitors and cardiomyocytes. Nat Methods. 8: 10371040.

Eschenhagen T, Fink C, Remmers U, Scholz H, Wattchow J, Weil J, Zimmermann W, Dohmen HH, Schafer H, Bishopric N, Wakatsuki T, Elson EL (1997) Three-dimensional reconstitution of embryonic cardiomyocytes in a collagen matrix: a new heart muscle model system. Faseb J. 11: 683-694.

Freund C, Ward-van Oostwaard D, Monshouwer-Kloots J, van den Brink S, van Rooijen M, Xu X, Zweigerdt R, Mummery C, Passier R (2008) Insulin redirects differentiation from cardiogenic mesoderm and endoderm to neuroectoderm in differentiating human embryonic stem cells. Stem Cells. 26: 724-733.

Gaussin V, Van de Putte T, Mishina Y, Hanks MC, Zwijsen A, Huylebroeck D, Behringer RR, Schneider MD (2002) Endocardial cushion and myocardial defects after cardiac myocyte-specific conditional deletion of the bone morphogenetic protein receptor ALK3. Proc Natl Acad Sci U S A. 99: 2878-2883.

Gilbert SF. (2000). Developmental Biology (6th edition ed.). Sunderland (MA): Sinauer Associates. 
Gjorevski N, Ranga A, Lutolf MP (2014) Bioengineering approaches to guide stem cell-based organogenesis. Development. 141: 1794-1804.

Godier-Furnemont AF, Tiburcy M, Wagner E, Dewenter M, Lammle S, El-Armouche A, Lehnart SE, Vunjak-Novakovic G, Zimmermann WH (2015) Physiologic forcefrequency response in engineered heart muscle by electromechanical stimulation. Biomaterials. 60: 82-91.

Gooch JL, Gorin Y, Zhang BX, Abboud HE (2004) Involvement of calcineurin in transforming growth factor-beta-mediated regulation of extracellular matrix accumulation. $J$ Biol Chem. 279: 15561-15570.

Goumans MJ, de Boer TP, Smits AM, van Laake LW, van Vliet P, Metz CHG, Korfage TH, Kats KP, Hochstenbach R, Pasterkamp G, Verhaar MC, van der Heyden MAG, de Kleijn D, Mummery CL, van Veen TAB, Sluijter JPG, Doevendans PA (2008) TGF- $\beta 1$ induces efficient differentiation of human cardiomyocyte progenitor cells into functional cardiomyocytes in vitro. Stem Cell Res. 1: 138-149.

Grant AO (2009) Cardiac ion channels. Circ Arrhythm Electrophysiol. 2: 185-194.

Groppe J, Greenwald J, Wiater E, Rodriguez-Leon J, Economides AN, Kwiatkowski W, Affolter M, Vale WW, Izpisua Belmonte JC, Choe S (2002) Structural basis of BMP signalling inhibition by the cystine knot protein Noggin. Nature. 420: 636-642.

Hall BK, Gillis JA (2013) Incremental evolution of the neural crest, neural crest cells and neural crest-derived skeletal tissues. J Anat. 222: 19-31.

Hao J, Daleo MA, Murphy CK, Yu PB, Ho JN, Hu J, Peterson RT, Hatzopoulos AK, Hong CC (2008) Dorsomorphin, a selective small molecule inhibitor of BMP signaling, promotes cardiomyogenesis in embryonic stem cells. PLoS One. 3.

Hao J, Ho JN, Lewis JA, Karim KA, Daniels RN, Gentry PR, Hopkins CR, Lindsley CW, Hong CC (2010) In vivo structure-activity relationship study of dorsomorphin analogues identifies selective VEGF and BMP inhibitors. ACS Chem Biol. 5: 245-253.

Harvey RP (2002) Patterning the vertebrate heart. Nat Rev Genet. 3: 544-556.

Hayashi M, Maeda S, Aburatani H, Kitamura K, Miyoshi H, Miyazono K, Imamura T (2008) Pitx2 prevents osteoblastic transdifferentiation of myoblasts by bone morphogenetic proteins. J Biol Chem. 283: 565-571.

He JQ, Ma Y, Lee Y, Thomson JA, Kamp TJ (2003) Human embryonic stem cells develop into multiple types of cardiac myocytes: action potential characterization. Circ Res. 93: 3239.

Herrero J, Valencia A, Dopazo J (2001) A hierarchical unsupervised growing neural network for clustering gene expression patterns. Bioinformatics. 17: 126-136. 
Hirt MN, Boeddinghaus J, Mitchell A, Schaaf S, Bornchen C, Muller C, Schulz H, Hubner N, Stenzig J, Stoehr A, Neuber C, Eder A, Luther PK, Hansen A, Eschenhagen T (2014) Functional improvement and maturation of rat and human engineered heart tissue by chronic electrical stimulation. J Mol Cell Cardiol. 74: 151-161.

Hoffman JI, Kaplan S (2002) The incidence of congenital heart disease. J Am Coll Cardiol. 39: 1890-1900.

Hoogaars WM, Barnett P, Moorman AF, Christoffels VM (2007) T-box factors determine cardiac design. Cell Mol Life Sci. 64: 646-660.

Hough SR, Laslett AL, Grimmond SB, Kolle G, Pera MF (2009) A Continuum of Cell States Spans Pluripotency and Lineage Commitment in Human Embryonic Stem Cells. PLoS One. 4.

Huang da W, Sherman BT, Lempicki RA (2009) Systematic and integrative analysis of large gene lists using DAVID bioinformatics resources. Nat Protoc. 4: 44-57.

Huch M, Bonfanti P, Boj SF, Sato T, Loomans CJM, van de Wetering M, Sojoodi M, Li VSW, Schuijers J, Gracanin A, Ringnalda F, Begthel H, Hamer K, Mulder J, van Es JH, de Koning E, Vries RGJ, Heimberg H, Clevers H (2013) Unlimited in vitro expansion of adult bi-potent pancreas progenitors through the Lgr5/R-spondin axis. Embo j. 32: 27082721.

Hudson J, Titmarsh D, Hidalgo A, Wolvetang E, Cooper-White J (2012) Primitive cardiac cells from human embryonic stem cells. Stem Cells Dev. 21: 1513-1523.

Jackman CP, Shadrin IY, Carlson AL, Bursac N (2015) Human Cardiac Tissue Engineering: From Pluripotent Stem Cells to Heart Repair. Curr Opin Chem Eng. 7: 57-64.

Jackson SA, Schiesser J, Stanley EG, Elefanty AG (2010) Differentiating embryonic stem cells pass through 'temporal windows' that mark responsiveness to exogenous and paracrine mesendoderm inducing signals. PLoS One. 5: e10706.

Jang EY, Choe ES, Hwang M, Kim SC, Lee JR, Kim SG, Jeon J, Buono RJ, Yang CH (2008) Isoliquiritigenin suppresses cocaine-induced extracellular dopamine release in rat brain through GABAB receptor. European Journal of Pharmacology. 587: 124-128.

Jiao K, Kulessa H, Tompkins K, Zhou Y, Batts L, Baldwin HS, Hogan BL (2003) An essential role of Bmp4 in the atrioventricular septation of the mouse heart. Genes Dev. 17: 23622367.

Kamakura T, Makiyama T, Sasaki K, Yoshida Y, Wuriyanghai Y, Chen J, Hattori T, Ohno S, Kita T, Horie M, Yamanaka S, Kimura T (2013) Ultrastructural maturation of humaninduced pluripotent stem cell-derived cardiomyocytes in a long-term culture. Circ J. 77: 1307-1314.

Karakikes I, Senyei GD, Hansen J, Kong CW, Azeloglu EU, Stillitano F, Lieu DK, Wang J, Ren L, Hulot JS, Iyengar R, Li RA, Hajjar RJ (2014) Small molecule-mediated directed 
differentiation of human embryonic stem cells toward ventricular cardiomyocytes. Stem Cells Transl Med. 3: 18-31.

Karaulanov E, Knochel W, Niehrs C (2004) Transcriptional regulation of BMP4 synexpression in transgenic Xenopus. Embo j. 23: 844-856.

Karthaus WR, Iaquinta PJ, Drost J, Gracanin A, van Boxtel R, Wongvipat J, Dowling CM, Gao D, Begthel H, Sachs N, Vries RG, Cuppen E, Chen Y, Sawyers CL, Clevers HC (2014) Identification of multipotent luminal progenitor cells in human prostate organoid cultures. Cell. 159: 163-175.

Kattman SJ, Witty AD, Gagliardi M, Dubois NC, Niapour M, Hotta A, Ellis J, Keller G (2011) Stage-Specific Optimization of Activin/Nodal and BMP Signaling Promotes Cardiac Differentiation of Mouse and Human Pluripotent Stem Cell Lines. Cell Stem Cell. 8: 228-240.

Kawabata M, Imamura T, Miyazono K (1998) Signal transduction by bone morphogenetic proteins. Cytokine Growth Factor Rev. 9: 49-61.

Kehat I, Gepstein A, Spira A, Itskovitz-Eldor J, Gepstein L (2002) High-Resolution Electrophysiological Assessment of Human Embryonic Stem Cell-Derived Cardiomyocytes. A Novel In Vitro Model for the Study of Conduction. Circ Res.

Kehat I, Kenyagin-Karsenti D, Snir M, Segev H, Amit M, Gepstein A, Livne E, Binah O, Itskovitz-Eldor J, Gepstein L (2001) Human embryonic stem cells can differentiate into myocytes with structural and functional properties of cardiomyocytes. The Journal of Clinical Investigation. 108: 407-414.

Kelly RG (2012) The second heart field. Curr Top Dev Biol. 100: 33-65.

Kelly RG, Brown NA, Buckingham ME (2001) The arterial pole of the mouse heart forms from Fgf10-expressing cells in pharyngeal mesoderm. Dev Cell. 1: 435-440.

Kennedy M, D'Souza SL, Lynch-Kattman M, Schwantz S, Keller G (2007) Development of the hemangioblast defines the onset of hematopoiesis in human ES cell differentiation cultures. Blood. 109: 2679-2687.

Kensah G, Roa Lara A, Dahlmann J, Zweigerdt R, Schwanke K, Hegermann J, Skvorc D, Gawol A, Azizian A, Wagner S, Maier LS, Krause A, Draeger G, Ochs M, Haverich A, Gruh I, Martin U (2012) Murine and human pluripotent stem cell-derived cardiac bodies form contractile myocardial tissue in vitro. Eur Heart $J$.

Kim D, Pertea G, Trapnell C, Pimentel H, Kelley R, Salzberg SL (2013) TopHat2: accurate alignment of transcriptomes in the presence of insertions, deletions and gene fusions. Genome Biol. 14: R36.

Kim MS, Horst A, Blinka S, Stamm K, Mahnke D, Schuman J, Gundry R, Tomita-Mitchell A, Lough J (2015) Activin-A and Bmp4 levels modulate cell type specification during CHIR-induced cardiomyogenesis. PLoS One. 10: e0118670. 
Kim RY, Robertson EJ, Solloway MJ (2001) Bmp6 and Bmp7 are required for cushion formation and septation in the developing mouse heart. Dev Biol. 235: 449-466.

Kruithof BP, Duim SN, Moerkamp AT, Goumans MJ (2012) TGFbeta and BMP signaling in cardiac cushion formation: lessons from mice and chicken. Differentiation. 84: 89-102.

Kwon C, Arnold J, Hsiao EC, Taketo MM, Conklin BR, Srivastava D (2007) Canonical Wnt signaling is a positive regulator of mammalian cardiac progenitors. Proc Natl Acad Sci U S A. 104: 10894-10899.

Laflamme MA, Chen KY, Naumova AV, Muskheli V, Fugate JA, Dupras SK, Reinecke H, Xu C, Hassanipour M, Police S, O'Sullivan C, Collins L, Chen Y, Minami E, Gill EA, Ueno S, Yuan C, Gold JD, Murry CE (2007) Cardiomyocytes derived from human embryonic stem cells in pro-survival factors enhance function of infarcted rat hearts. Nat Biotech. 25: 1015-1024.

Lancaster MA, Knoblich JA (2014) Organogenesis in a dish: Modeling development and disease using organoid technologies. Science. 345.

Lancaster MA, Renner M, Martin CA, Wenzel D, Bicknell LS, Hurles ME, Homfray T, Penninger JM, Jackson AP, Knoblich JA (2013) Cerebral organoids model human brain development and microcephaly. Nature. 501: 373-379.

Langer R, Vacanti JP (1993) Tissue engineering. Science. 260: 920-926.

Laslett AL, Grimmond S, Gardiner B, Stamp L, Lin A, Hawes SM, Wormald S, NikolicPaterson D, Haylock D, Pera MF (2007) Transcriptional analysis of early lineage commitment in human embryonic stem cells. BMC Dev Biol. 7: 12.

Lawson KA, Dunn NR, Roelen BAJ, Zeinstra LM, Davis AM, Wright CVE, Korving J, Hogan BLM (1999) Bmp4 is required for the generation of primordial germ cells in the mouse embryo. Genes Dev. 13: 424-436.

Lee LH, Peerani R, Ungrin M, Joshi C, Kumacheva E, Zandstra P (2009) Micropatterning of human embryonic stem cells dissects the mesoderm and endoderm lineages. Stem Cell Res. 2: 155-162.

Lenhart KF, Holtzman NG, Williams JR, Burdine RD (2013) Integration of nodal and BMP signals in the heart requires FoxH1 to create left-right differences in cell migration rates that direct cardiac asymmetry. PLoS Genet. 9: e1003109.

Li P, Cavallero S, Gu Y, Chen TH, Hughes J, Hassan AB, Bruning JC, Pashmforoush M, Sucov HM (2011) IGF signaling directs ventricular cardiomyocyte proliferation during embryonic heart development. Development. 138: 1795-1805.

Li TS, Hayashi M, Ito H, Furutani A, Murata T, Matsuzaki M, Hamano K (2005) Regeneration of infarcted myocardium by intramyocardial implantation of ex vivo transforming growth factor-beta-preprogrammed bone marrow stem cells. Circulation. 111: 24382445. 
Lian X, Bao X, Al-Ahmad A, Liu J, Wu Y, Dong W, Dunn KK, Shusta EV, Palecek SP (2014) Efficient Differentiation of Human Pluripotent Stem Cells to Endothelial Progenitors via Small-Molecule Activation of WNT Signaling. Stem Cell Reports. 3: 804-816.

Lian X, Bao X, Zilberter M, Westman M, Fisahn A, Hsiao C, Hazeltine LB, Dunn KK, Kamp TJ, Palecek SP (2015) Chemically defined, albumin-free human cardiomyocyte generation. Nat Meth. 12: 595-596.

Lian X, Zhang J, Azarin SM, Zhu K, Hazeltine LB, Bao X, Hsiao C, Kamp TJ, Palecek SP (2013) Directed cardiomyocyte differentiation from human pluripotent stem cells by modulating Wnt/beta-catenin signaling under fully defined conditions. Nat Protoc. 8: $162-175$.

Lian X, Zhang J, Zhu K, Kamp TJ, Palecek SP (2013) Insulin inhibits cardiac mesoderm, not mesendoderm, formation during cardiac differentiation of human pluripotent stem cells and modulation of canonical Wnt signaling can rescue this inhibition. Stem Cells. 31: 447-457.

Liang X, Wang G, Lin L, Lowe J, Zhang Q, Bu L, Chen Y, Chen J, Sun Y, Evans SM (2013) HCN4 dynamically marks the first heart field and conduction system precursors. Circ Res. 113: 399-407.

Liaw NY, Zimmermann WH (2016) Mechanical stimulation in the engineering of heart muscle. Adv Drug Deliv Rev. 96: 156-160.

Lim WF, Inoue-Yokoo T, Tan KS, Lai MI, Sugiyama D (2013) Hematopoietic cell differentiation from embryonic and induced pluripotent stem cells. Stem Cell Research \& Therapy. 4: 71-71.

Liu W, Selever J, Wang D, Lu MF, Moses KA, Schwartz RJ, Martin JF (2004) Bmp4 signaling is required for outflow-tract septation and branchial-arch artery remodeling. Proc Natl Acad Sci U S A. 101: 4489-4494.

Lundy SD, Zhu WZ, Regnier M, Laflamme MA (2013) Structural and functional maturation of cardiomyocytes derived from human pluripotent stem cells. Stem Cells Dev. 22: 19912002.

Mandela P, Chandley M, Xu YY, Zhu MY, Ordway GA (2010) Reserpine-induced reduction in norepinephrine transporter function requires catecholamine storage vesicles. Neurochem Int. 56: 760-767.

Martin-Puig S, Wang Z, Chien KR (2008) Lives of a heart cell: tracing the origins of cardiac progenitors. Cell Stem Cell. 2: 320-331.

McCulley DJ, Black BL (2012) Transcription factor pathways and congenital heart disease. Curr Top Dev Biol. 100: 253-277. 
McDevitt TC, Laflamme MA, Murry CE (2005) Proliferation of cardiomyocytes derived from human embryonic stem cells is mediated via the IGF/PI 3-kinase/Akt signaling pathway. J Mol Cell Cardiol. 39: 865-873.

Metzger RR, Brown JM, Sandoval V, Rau KS, Elwan MA, Miller GW, Hanson GR, Fleckenstein AE (2002) Inhibitory effect of reserpine on dopamine transporter function. Eur J Pharmacol. 456: 39-43.

Misfeldt AM, Boyle SC, Tompkins KL, Bautch VL, Labosky PA, Baldwin HS (2009) Endocardial cells are a distinct endothelial lineage derived from Flk1+ multipotent cardiovascular progenitors. Dev Biol. 333: 78-89.

Mishina Y, Suzuki A, Ueno N, Behringer RR (1995) Bmpr encodes a type I bone morphogenetic protein receptor that is essential for gastrulation during mouse embryogenesis. Genes Dev. 9: 3027-3037.

Miyazono K, Kamiya Y, Morikawa M (2010) Bone morphogenetic protein receptors and signal transduction. Journal of Biochemistry. 147: 35-51.

Miyazono K, Miyazawa K (2002) Id: a target of BMP signaling. Sci STKE. 24.

Mjaatvedt CH, Nakaoka T, Moreno-Rodriguez R, Norris RA, Kern MJ, Eisenberg CA, Turner D, Markwald RR (2001) The outflow tract of the heart is recruited from a novel heartforming field. Dev Biol. 238: 97-109.

Molinoff PB, Axelrod J (1971) Biochemistry of catecholamines. Annu Rev Biochem. 40: 465500.

Moorman A, Webb S, Brown NA, Lamers W, Anderson RH (2003) Development of the heart: (1) formation of the cardiac chambers and arterial trunks. Heart. 89: 806-814.

Moretti A, Caron L, Nakano A, Lam JT, Bernshausen A, Chen Y, Qyang Y, Bu L, Sasaki M, Martin-Puig S, Sun Y, Evans SM, Laugwitz KL, Chien KR (2006) Multipotent embryonic isl1+ progenitor cells lead to cardiac, smooth muscle, and endothelial cell diversification. Cell. 127: 1151-1165.

Muller-Pillasch F, Gress TM, Yamaguchi H, Geng M, Adler G, Menke A (1997) The influence of transforming growth factor beta 1 on the expression of genes coding for matrix metalloproteinases and tissue inhibitors of metalloproteinases during regeneration from cerulein-induced pancreatitis. Pancreas. 15: 168-175.

Mummery C, Ward-van Oostwaard D, Doevendans P, Spijker R, van den Brink S, Hassink R, van der Heyden M, Opthof T, Pera M, de la Riviere AB, Passier R, Tertoolen L (2003) Differentiation of human embryonic stem cells to cardiomyocytes: role of coculture with visceral endoderm-like cells. Circulation. 107: 2733-2740.

Mummery CL, Zhang J, Ng ES, Elliott DA, Elefanty AG, Kamp TJ (2012) Differentiation of Human Embryonic Stem Cells and Induced Pluripotent Stem Cells to Cardiomyocytes. Circ Res. 111: 344-358. 
Murry CE, Keller G (2008) Differentiation of embryonic stem cells to clinically relevant populations: lessons from embryonic development. Cell. 132: 661-680.

Naito AT, Akazawa H, Takano H, Minamino T, Nagai T, Aburatani H, Komuro I (2005) Phosphatidylinositol 3-kinase-Akt pathway plays a critical role in early cardiomyogenesis by regulating canonical Wnt signaling. Circ Res. 97: 144-151.

Naito AT, Shiojima I, Akazawa H, Hidaka K, Morisaki T, Kikuchi A, Komuro I (2006) Developmental stage-specific biphasic roles of $\mathrm{Wnt} / \beta$-catenin signaling in cardiomyogenesis and hematopoiesis. Proceedings of the National Academy of Sciences. 103: 19812-19817.

Naito H, Melnychenko I, Didie M, Schneiderbanger K, Schubert P, Rosenkranz S, Eschenhagen T, Zimmermann WH (2006) Optimizing engineered heart tissue for therapeutic applications as surrogate heart muscle. Circulation. 114: I72-78.

Nakamura T, Miller D, Ruoslahti E, Border WA (1992) Production of extracellular matrix by glomerular epithelial cells is regulated by transforming growth factor-beta 1. Kidney Int. 41: 1213-1221.

Noguchi C, Yang J, Sakamoto K, Maeda R, Takahashi K, Takasugi H, Ono T, Murakawa M, Kimura J (2008) Inhibitory effects of isoliquiritigenin and licorice extract on voltagedependent $\mathrm{K}(+)$ currents in H9c2 cells. J Pharmacol Sci. 108: 439-445.

Noisa P, Ramasamy TS, Lamont FR, Yu JSL, Sheldon MJ, Russell A, Jin X, Cui W (2012) Identification and Characterisation of the Early Differentiating Cells in Neural Differentiation of Human Embryonic Stem Cells. PLoS One. 7: e37129.

Norstrom A, Akesson K, Hardarson T, Hamberger L, Bjorquist P, Sartipy P (2006) Molecular and pharmacological properties of human embryonic stem cell-derived cardiomyocytes. Exp Biol Med. 231: 1753-1762.

Nostro MC, Cheng X, Keller GM, Gadue P (2008) Wnt, activin, and BMP signaling regulate distinct stages in the developmental pathway from embryonic stem cells to blood. Cell Stem Cell. 2: 60-71.

Nunes SS, Miklas JW, Liu J, Aschar-Sobbi R, Xiao Y, Zhang B, Jiang J, Masse S, Gagliardi M, Hsieh A, Thavandiran N, Laflamme MA, Nanthakumar K, Gross GJ, Backx PH, Keller G, Radisic M (2013) Biowire: a platform for maturation of human pluripotent stem cellderived cardiomyocytes. Nat Methods. 10: 781-787.

Okita K, Yamanaka S (2006) Intracellular signaling pathways regulating pluripotency of embryonic stem cells. Curr Stem Cell Res Ther. 1: 103-111.

Orlova VV, Chuva de Sousa Lopes S, Valdimarsdottir G (2016) BMP-SMAD signaling: From pluripotent stem cells to cardiovascular commitment. Cytokine Growth Factor Rev. 27: 55-63. 
Paige SL, Osugi T, Afanasiev OK, Pabon L, Reinecke H, Murry CE (2010) Endogenous Wnt/beta-catenin signaling is required for cardiac differentiation in human embryonic stem cells. PLoS One. 5.

Paige SL, Plonowska K, Xu A, Wu SM (2015) Molecular regulation of cardiomyocyte differentiation. Circ Res. 116: 341-353.

Park EJ, Ogden LA, Talbot A, Evans S, Cai CL, Black BL, Frank DU, Moon AM (2006) Required, tissue-specific roles for Fgf8 in outflow tract formation and remodeling. Development. 133: 2419-2433.

Peterson S, Loring J. (2012). Human Stem Cell Manual: A Laboratory Guide (Second Edition ed.): ELSEVIER.

Qin BY, Chacko BM, Lam SS, de Caestecker MP, Correia JJ, Lin K (2001) Structural Basis of Smad1 Activation by Receptor Kinase Phosphorylation. Mol Cell. 8: 1303-1312.

Radisic M, Park H, Shing H, Consi T, Schoen FJ, Langer R, Freed LE, Vunjak-Novakovic G (2004) Functional assembly of engineered myocardium by electrical stimulation of cardiac myocytes cultured on scaffolds. Proc Natl Acad Sci U S A. 101: 18129-18134.

Rajala K, Pekkanen-Mattila M, Aalto-Set K (2011) Cardiac Differentiation of Pluripotent Stem Cells. Stem Cells International. 2011.

Rana MS, Christoffels VM, Moorman AF (2013) A molecular and genetic outline of cardiac morphogenesis. Acta Physiol (Oxf). 207: 588-615.

Rana MS, Theveniau-Ruissy M, De Bono C, Mesbah K, Francou A, Rammah M, Dominguez JN, Roux M, Laforest B, Anderson RH, Mohun T, Zaffran S, Christoffels VM, Kelly RG (2014) Tbx 1 coordinates addition of posterior second heart field progenitor cells to the arterial and venous poles of the heart. Circ Res. 115: 790-799.

Reynolds ES (1963) The use of lead citrate at high $\mathrm{pH}$ as an electron-opaque stain in electron microscopy. J Cell Biol. 17: 208-212.

Rivera-Feliciano J, Tabin CJ (2006) Bmp2 instructs cardiac progenitors to form the heart-valveinducing field. Dev Biol. 295: 580-588.

Saeed AI, Sharov V, White J, Li J, Liang W, Bhagabati N, Braisted J, Klapa M, Currier T, Thiagarajan M, Sturn A, Snuffin M, Rezantsev A, Popov D, Ryltsov A, Kostukovich E, Borisovsky I, Liu Z, Vinsavich A, Trush V, Quackenbush J (2003) TM4: a free, opensource system for microarray data management and analysis. Biotechniques. 34: 374378.

Samad TA, Rebbapragada A, Bell E, Zhang Y, Sidis Y, Jeong S, Campagna JA, Perusini S, Fabrizio DA, Schneyer AL, Lin HY, Brivanlou AH, Attisano L, Woolf CJ (2005) DRAGON, a Bone Morphogenetic Protein Co-receptor. Journal of Biological Chemistry. 280: 14122-14129. 
Sartiani L, Bettiol E, Stillitano F, Mugelli A, Cerbai E, Jaconi ME (2007) Developmental changes in cardiomyocytes differentiated from human embryonic stem cells: a molecular and electrophysiological approach. Stem Cells. 25: 1136-1144.

Sasaki T, Ito Y, Bringas P, Jr., Chou S, Urata MM, Slavkin H, Chai Y (2006) TGFbetamediated FGF signaling is crucial for regulating cranial neural crest cell proliferation during frontal bone development. Development. 133: 371-381.

Schaaf S, Shibamiya A, Mewe M, Eder A, Stohr A, Hirt MN, Rau T, Zimmermann W-H, Conradi L, Eschenhagen T, Hansen A (2011) Human Engineered Heart Tissue as a Versatile Tool in Basic Research and Preclinical Toxicology. PLoS One. 6: e26397.

Schram G, Pourrier M, Melnyk P, Nattel S (2002) Differential distribution of cardiac ion channel expression as a basis for regional specialization in electrical function. Circ Res. 90: 939-950.

Schwach V, Passier R (2016) Generation and purification of human stem cell-derived cardiomyocytes. Differentiation.

Sebald W, Nickel J, Zhang JL, Mueller TD (2004) Molecular recognition in bone morphogenetic protein (BMP)/receptor interaction. Biol Chem. 385: 697-710.

Shanks N, Greek R, Greek J (2009) Are animal models predictive for humans? Philos Ethics Humanit Med. 4: 2.

Shimizu T, Yamato M, Isoi Y, Akutsu T, Setomaru T, Abe K, Kikuchi A, Umezu M, Okano T (2002) Fabrication of pulsatile cardiac tissue grafts using a novel 3-dimensional cell sheet manipulation technique and temperature-responsive cell culture surfaces. Circ Res. 90: e40.

Simmler C, Hajirahimkhan A, Lankin DC, Bolton JL, Jones T, Soejarto DD, Chen SN, Pauli GF (2013) Dynamic residual complexity of the isoliquiritigenin-liquiritigenin interconversion during bioassay. J Agric Food Chem. 61: 2146-2157.

Skottman H, Mikkola M, Lundin K, Olsson C, Stromberg AM, Tuuri T, Otonkoski T, Hovatta O, Lahesmaa R (2005) Gene expression signatures of seven individual human embryonic stem cell lines. Stem Cells. 23: 1343-1356.

Small EM, Krieg PA (2004) Molecular regulation of cardiac chamber-specific gene expression. Trends Cardiovasc Med. 14: 13-18.

Snir M, Kehat I, Gepstein A, Coleman R, Itskovitz-Eldor J, Livne E, Gepstein L (2003) Assessment of the ultrastructural and proliferative properties of human embryonic stem cell-derived cardiomyocytes. Am J Physiol Heart Circ Physiol. 285: H2355-2363.

Soong PL, Tiburcy M, Zimmermann WH (2012) Cardiac differentiation of human embryonic stem cells and their assembly into engineered heart muscle. Curr Protoc Cell Biol. Chapter 23: Unit23.28. 
Souchelnytskyi S, Moustakas A, Heldin CH. (2002). TGF-beta signaling from a threedimensional perspective: insight into selection of partners: Trends Cell Biol. 2002 Jul;12(7):304-7.

Streckfuss-Bomeke K, Wolf F, Azizian A, Stauske M, Tiburcy M, Wagner S, Hubscher D, Dressel R, Chen S, Jende J, Wulf G, Lorenz V, Schon MP, Maier LS, Zimmermann WH, Hasenfuss G, Guan K (2013) Comparative study of human-induced pluripotent stem cells derived from bone marrow cells, hair keratinocytes, and skin fibroblasts. Eur Heart J. 34: 2618-2629.

Sugiyama D, Kulkeaw K, Mizuochi C (2013) TGF-beta-1 up-regulates extra-cellular matrix production in mouse hepatoblasts. Mechanisms of Development. 130: 195-206.

Takahashi K, Tanabe K, Ohnuki M, Narita M, Ichisaka T, Tomoda K, Yamanaka S (2007) Induction of pluripotent stem cells from adult human fibroblasts by defined factors. Cell. 131: 861-872.

Takasato M, Er PX, Becroft M, Vanslambrouck JM, Stanley EG, Elefanty AG, Little MH (2014) Directing human embryonic stem cell differentiation towards a renal lineage generates a self-organizing kidney. Nat Cell Biol. 16: 118-126.

Takebe T, Sekine K, Enomura M, Koike H, Kimura M, Ogaeri T, Zhang R, Ueno Y, Zheng Y, Koike N, Aoyama S, Adachi Y, Taniguchi H (2013) Vascularized and functional human liver from an iPSC-derived organ bud transplant. Nature. 499: 481-484.

Takei S, Ichikawa H, Johkura K, Mogi A, No H, Yoshie S, Tomotsune D, Sasaki K (2009) Bone morphogenetic protein-4 promotes induction of cardiomyocytes from human embryonic stem cells in serum-based embryoid body development. Am J Physiol Heart Circ Physiol. 296: 10.

Takeuchi JK, Ohgi M, Koshiba-Takeuchi K, Shiratori H, Sakaki I, Ogura K, Saijoh Y, Ogura T (2003) Tbx5 specifies the left/right ventricles and ventricular septum position during cardiogenesis. Development. 130: 5953-5964.

Tam PP, Loebel DA (2007) Gene function in mouse embryogenesis: get set for gastrulation. Nat Rev Genet. 8: 368-381.

Thomson JA, Itskovitz-Eldor J, Shapiro SS, Waknitz MA, Swiergiel JJ, Marshall VS, Jones JM (1998) Embryonic stem cell lines derived from human blastocysts. Science. 282: 11451147.

Tiburcy M, Didie M, Boy O, Christalla P, Doeker S, Naito H, Karikkineth BC, El-Armouche A, Grimm M, Nose M, Eschenhagen T, Zieseniss A, Katschinksi DM, Hamdani N, Linke WA, Yin X, Mayr M, Zimmermann W-H (2011) Terminal Differentiation, Advanced Organotypic Maturation, and Modeling of Hypertrophic Growth in Engineered Heart Tissue / Novelty and Significance. Circ Res. 109: 1105-1114.

Tiburcy M, Meyer T, Soong PL, Zimmermann WH (2014) Collagen-based engineered heart muscle. Methods Mol Biol. 1181: 167-176. 
Tran TH, Wang X, Browne C, Zhang Y, Schinke M, Izumo S, Burcin M (2009) Wnt3a-induced mesoderm formation and cardiomyogenesis in human embryonic stem cells. Stem Cells. 27: 1869-1878.

Trapnell C, Roberts A, Goff L, Pertea G, Kim D, Kelley DR, Pimentel H, Salzberg SL, Rinn JL, Pachter L (2012) Differential gene and transcript expression analysis of RNA-seq experiments with TopHat and Cufflinks. Nat Protoc. 7: 562-578.

Tremblay KD, Dunn NR, Robertson EJ (2001) Mouse embryos lacking Smad1 signals display defects in extra-embryonic tissues and germ cell formation. Development. 128: 36093621.

Truksa J, Lee P, Beutler E (2009) Two BMP responsive elements, STAT, and bZIP/HNF4/COUP motifs of the hepcidin promoter are critical for BMP, SMAD1, and HJV responsiveness. Blood. 113: 688-695.

Tulloch NL, Muskheli V, Razumova MV, Korte FS, Regnier M, Hauch KD, Pabon L, Reinecke H, Murry CE (2011) Growth of engineered human myocardium with mechanical loading and vascular coculture. Circ Res. 109: 47-59.

Uhlen M, Fagerberg L, Hallstrom BM, Lindskog C, Oksvold P, Mardinoglu A, Sivertsson A, Kampf C, Sjostedt E, Asplund A, Olsson I, Edlund K, Lundberg E, Navani S, Szigyarto CA, Odeberg J, Djureinovic D, Takanen JO, Hober S, Alm T, Edqvist PH, Berling H, Tegel H, Mulder J, Rockberg J, Nilsson P, Schwenk JM, Hamsten M, von Feilitzen K, Forsberg M, Persson L, Johansson F, Zwahlen M, von Heijne G, Nielsen J, Ponten F (2015) Proteomics. Tissue-based map of the human proteome. Science. 347: 1260419.

Uosaki H, Fukushima H, Takeuchi A, Matsuoka S, Nakatsuji N, Yamanaka S, Yamashita JK (2011) Efficient and scalable purification of cardiomyocytes from human embryonic and induced pluripotent stem cells by VCAM1 surface expression. PLoS One. 6: e23657.

Urist MR (1965) Bone: formation by autoinduction. Science. 150: 893-899.

van den Ameele J, Tiberi L, Bondue A, Paulissen C, Herpoel A, Iacovino M, Kyba M, Blanpain C, Vanderhaeghen P (2012) Eomesodermin induces Mesp1 expression and cardiac differentiation from embryonic stem cells in the absence of Activin. EMBO Rep. 13: 355-362.

Verma V, Purnamawati K, Manasi, Shim W (2013) Steering signal transduction pathway towards cardiac lineage from human pluripotent stem cells: a review. Cell Signal. 25: 1096-1107.

Vrijens K, Lin W, Cui J, Farmer D, Low J, Pronier E, Zeng FY, Shelat AA, Guy K, Taylor MR, Chen T, Roussel MF (2013) Identification of Small Molecule Activators of BMP Signaling. PLoS One. 8.

Waldo KL, Kumiski DH, Wallis KT, Stadt HA, Hutson MR, Platt DH, Kirby ML (2001) Conotruncal myocardium arises from a secondary heart field. Development. 128: 31793188. 
Wang H, Hao J, Hong CC (2011) Cardiac induction of embryonic stem cells by a small molecule inhibitor of Wnt/beta-catenin signaling. ACS Chem Biol. 6: 192-197.

Wang J, Greene SB, Bonilla-Claudio M, Tao Y, Zhang J, Bai Y, Huang Z, Black BL, Wang F, Martin JF (2010) Bmp signaling regulates myocardial differentiation from cardiac progenitors through a MicroRNA-mediated mechanism. Dev Cell. 19: 903-912.

Wang RN, Green J, Wang Z, Deng Y, Qiao M, Peabody M, Zhang Q, Ye J, Yan Z, Denduluri S, Idowu O, Li M, Shen C, Hu A, Haydon RC, Kang R, Mok J, Lee MJ, Luu HL, Shi LL (2014) Bone Morphogenetic Protein (BMP) signaling in development and human diseases. Genes Dis. 1: 87-105.

Watanabe Y, Zaffran S, Kuroiwa A, Higuchi H, Ogura T, Harvey RP, Kelly RG, Buckingham M (2012) Fibroblast growth factor 10 gene regulation in the second heart field by Tbx 1 , Nkx2-5, and Islet1 reveals a genetic switch for down-regulation in the myocardium. Proc Natl Acad Sci U S A. 109: 18273-18280.

Willems E, Bushway PJ, Mercola M (2009) Natural and synthetic regulators of embryonic stem cell cardiogenesis. Pediatr Cardiol. 30: 635-642.

Willems E, Spiering S, Davidovics H, Lanier M, Xia Z, Dawson M, Cashman J, Mercola M (2011) Small-molecule inhibitors of the Wnt pathway potently promote cardiomyocytes from human embryonic stem cell-derived mesoderm. Circ Res. 109: 360-364.

Witty AD, Mihic A, Tam RY, Fisher SA, Mikryukov A, Shoichet MS, Li RK, Kattman SJ, Keller G (2014) Generation of the epicardial lineage from human pluripotent stem cells. Nat Biotechnol. 32: 1026-1035.

Wozney JM, Rosen V, Celeste AJ, Mitsock LM, Whitters MJ, Kriz RW, Hewick RM, Wang EA (1988) Novel regulators of bone formation: molecular clones and activities. Science. 242: $1528-1534$.

Wu J, Kubota J, Hirayama J, Nagai Y, Nishina S, Yokoi T, Asaoka Y, Seo J, Shimizu N, Kajiho H, Watanabe T, Azuma N, Katada T, Nishina H (2010) p38 Mitogen-activated protein kinase controls a switch between cardiomyocyte and neuronal commitment of murine embryonic stem cells by activating myocyte enhancer factor 2C-dependent bone morphogenetic protein 2 transcription. Stem Cells Dev. 19: 1723-1734.

Wu SM, Fujiwara Y, Cibulsky SM, Clapham DE, Lien CL, Schultheiss TM, Orkin SH (2006) Developmental origin of a bipotential myocardial and smooth muscle cell precursor in the mammalian heart. Cell. 127: 1137-1150.

Xia Y, Yu PB, Sidis Y, Beppu H, Bloch KD, Schneyer AL, Lin HY (2007) Repulsive Guidance Molecule RGMa Alters Utilization of Bone Morphogenetic Protein (BMP) Type II Receptors by BMP2 and BMP4. Journal of Biological Chemistry. 282: 18129-18140.

Xin M, Kim Y, Sutherland LB, Qi X, McAnally J, Schwartz RJ, Richardson JA, Bassel-Duby R, Olson EN (2011) Regulation of insulin-like growth factor signaling by Yap governs cardiomyocyte proliferation and embryonic heart size. Sci Signal. 4: ra70. 
Xu XQ, Graichen R, Soo SY, Balakrishnan T, Rahmat SN, Sieh S, Tham SC, Freund C, Moore J, Mummery C, Colman A, Zweigerdt R, Davidson BP (2008) Chemically defined medium supporting cardiomyocyte differentiation of human embryonic stem cells. Differentiation. 76: 958-970.

Xu XQ, Soo SY, Sun W, Zweigerdt R (2009) Global expression profile of highly enriched cardiomyocytes derived from human embryonic stem cells. Stem Cells. 27: 2163-2174.

Yamamoto H, Kamegaya E, Hagino Y, Imai K, Fujikawa A, Tamura K, Enokiya T, Yamamoto T, Takeshima T, Koga H, Uhl GR, Ikeda K, Sora I (2007) Genetic deletion of vesicular monoamine transporter-2 (VMAT2) reduces dopamine transporter activity in mesencephalic neurons in primary culture. Neurochem Int. 51: 237-244.

Yang L, Cai CL, Lin L, Qyang Y, Chung C, Monteiro RM, Mummery CL, Fishman GI, Cogen A, Evans S (2006) Isl1Cre reveals a common Bmp pathway in heart and limb development. Development. 133: 1575-1585.

Yang L, Soonpaa MH, Adler ED, Roepke TK, Kattman SJ, Kennedy M, Henckaerts E, Bonham K, Abbott GW, Linden RM, Field LJ, Keller GM (2008) Human cardiovascular progenitor cells develop from a KDR+ embryonic-stem-cell-derived population. Nature. 453: 524-528.

Yao S, Chen S, Clark J, Hao E, Beattie GM, Hayek A, Ding S (2006) Long-term self-renewal and directed differentiation of human embryonic stem cells in chemically defined conditions. Proceedings of the National Academy of Sciences. 103: 6907-6912.

Yin X, Mead BE, Safaee H, Langer R, Karp JM, Levy O (2016) Engineering Stem Cell Organoids. Cell Stem Cell. 18: 25-38.

Ying QL, Stavridis M, Griffiths D, Li M, Smith A (2003) Conversion of embryonic stem cells into neuroectodermal precursors in adherent monoculture. Nat Biotechnol. 21: 183-186.

Yoon BS, Yoo SJ, Lee JE, You S, Lee HT, Yoon HS (2006) Enhanced differentiation of human embryonic stem cells into cardiomyocytes by combining hanging drop culture and 5azacytidine treatment. Differentiation. 74: 149-159.

Yu J, Vodyanik MA, Smuga-Otto K, Antosiewicz-Bourget J, Frane JL, Tian S, Nie J, Jonsdottir GA, Ruotti V, Stewart R, Slukvin, II, Thomson JA (2007) Induced pluripotent stem cell lines derived from human somatic cells. Science. 318: 1917-1920.

Yu PB, Hong CC, Sachidanandan C, Babitt JL, Deng DY, Hoyng SA, Lin HY, Bloch KD, Peterson RT (2008) Dorsomorphin inhibits BMP signals required for embryogenesis and iron metabolism. Nat Chem Biol. 4: 33-41.

Yuasa S, Itabashi Y, Koshimizu U, Tanaka T, Sugimura K, Kinoshita M, Hattori F, Fukami S, Shimazaki T, Ogawa S, Okano H, Fukuda K. (2005). Transient inhibition of BMP signaling by Noggin induces cardiomyocyte differentiation of mouse embryonic stem cells: Nat Biotechnol. 2005 May;23(5):607-11. Epub 2005 May 1. 
Zaffran S, Kelly RG (2012) New developments in the second heart field. Differentiation. 84: 1724.

Zeidler S, Meckbach C, Tacke R, Raad FS, Roa A, Uchida S, Zimmermann WH, Wingender E, Gultas M (2016) Computational Detection of Stage-Specific Transcription Factor Clusters during Heart Development. Front Genet. 7: 33.

Zhang D, Shadrin IY, Lam J, Xian HQ, Snodgrass HR, Bursac N (2013) Tissue-engineered cardiac patch for advanced functional maturation of human ESC-derived cardiomyocytes. Biomaterials. 34: 5813-5820.

Zhang P, Li J, Tan Z, Wang C, Liu T, Chen L, Yong J, Jiang W, Sun X, Du L, Ding M, Deng H (2008) Short-term BMP-4 treatment initiates mesoderm induction in human embryonic stem cells. Blood. 111: 1933-1941.

Zhang Q, Jiang J, Han P, Yuan Q, Zhang J, Zhang X, Xu Y, Cao H, Meng Q, Chen L, Tian T, Wang X, Li P, Hescheler J, Ji G, Ma Y (2011) Direct differentiation of atrial and ventricular myocytes from human embryonic stem cells by alternating retinoid signals. Cell Res. 21: 579-587.

Zhou B, Xing C (2015) Diverse Molecular Targets for Chalcones with Varied Bioactivities. Med Chem (Los Angeles). 5: 388-404.

Zhu MX, Zhao JY, Chen GA (2013) Mesoderm is committed to hemato-endothelial and cardiac lineages in human embryoid bodies by sequential exposure to cytokines. Exp Cell Res. 319: 21-34.

Zimmermann W-H, Fink C, Kralisch D, Remmers U, Weil J, Eschenhagen T (2000) Threedimensional engineered heart tissue from neonatal rat cardiac myocytes. Biotechnol Bioeng. 68: 106-114.

Zimmermann W-H, Melnychenko I, Wasmeier G, Didie M, Naito H, Nixdorff U, Hess A, Budinsky L, Brune K, Michaelis B, Dhein S, Schwoerer A, Ehmke H, Eschenhagen T (2006) Engineered heart tissue grafts improve systolic and diastolic function in infarcted rat hearts. Nat Med. 12: 452-458.

Zimmermann W-H, Schneiderbanger K, Schubert P, Didie M, Maenzel F, Heubach JF, Kostin S, Neuhuber WL, Eschenhagen T (2002) Tissue Engineering of a Differentiated Cardiac Muscle Construct. Circ Res. 90: 223-230.

Zimmermann WH (2011) Embryonic and embryonic-like stem cells in heart muscle engineering. J Mol Cell Cardiol. 50: 320-326. 


\section{Appendix}

\section{$\underline{\text { Cell culture reagents }}$}

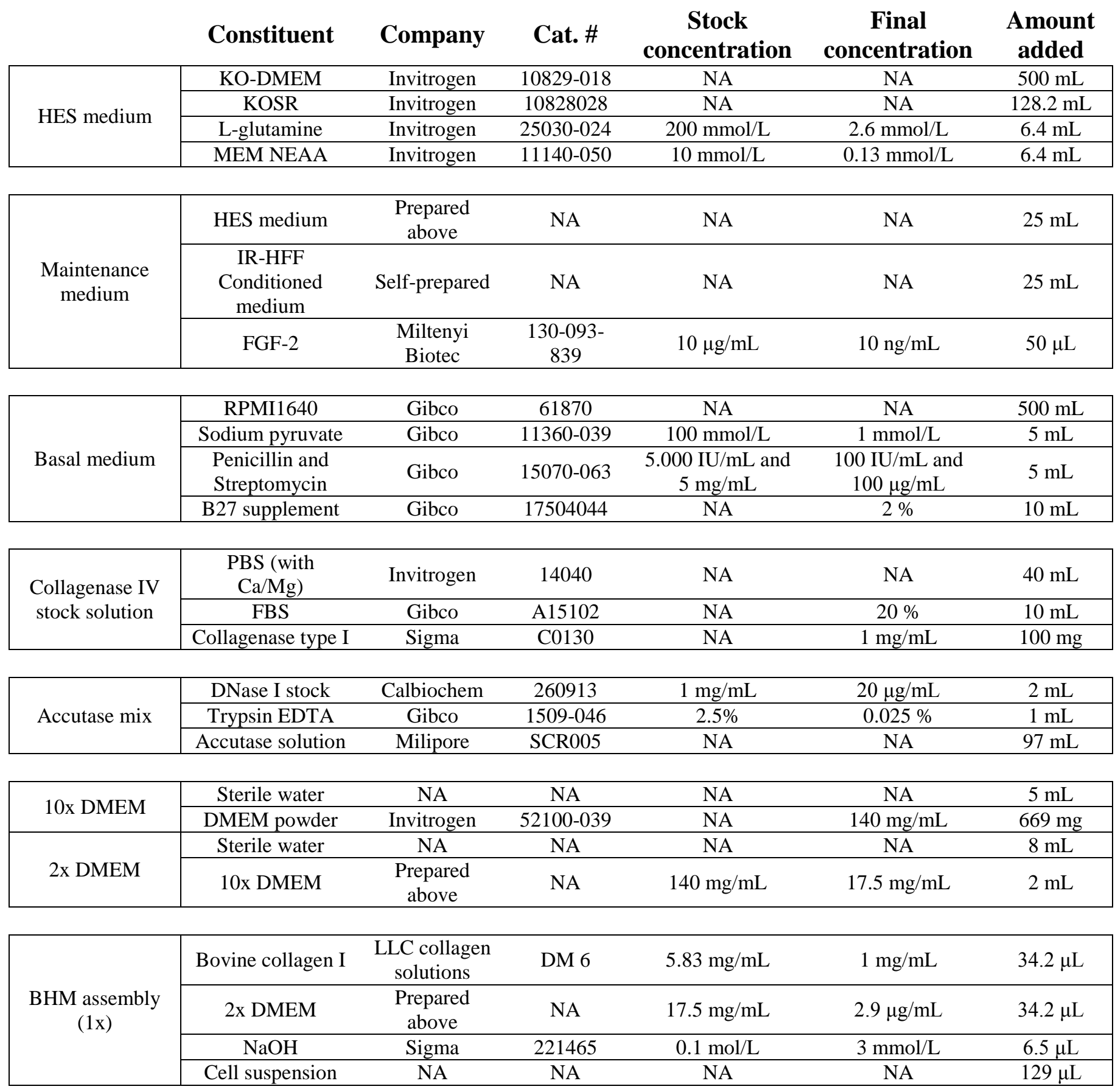


$R P M I$ and Iscove media components

\begin{tabular}{|c|c|c|c|}
\hline Components (mg/mL) & $\begin{array}{l}\text { Molecular Weight } \\
(\mathrm{g} / \mathrm{mol})\end{array}$ & $\begin{array}{l}\text { RPMI1640 } \\
\text { GlutaMax }^{\mathrm{TM}}\end{array}$ & $\begin{array}{l}\text { Iscove } \\
\text { medium }\end{array}$ \\
\hline \multicolumn{4}{|l|}{ Aminoacids } \\
\hline Glycine & 75 & 10 & 30 \\
\hline L-Alanyl-Glutamine & 217 & 446 & 584 \\
\hline L-Alanine & 89 & - & 25 \\
\hline L-Arginine & 174 & 200 & 84 \\
\hline L-Asparagine & 132 & 50 & 25 \\
\hline L-Aspartic acid & 133 & 20 & 30 \\
\hline L-Cystine & 240 & 50 & - \\
\hline L-Glutamic Acid & 147 & 20 & 75 \\
\hline L-Histidine & 155 & 15 & 42 \\
\hline L-Hydroxyproline & 131 & 20 & - \\
\hline L-Isoleucine & 131 & 50 & 105 \\
\hline L-Leucine & 131 & 50 & 105 \\
\hline L-Lysine hydrochloride & 183 & 40 & 146 \\
\hline L-Methionine & 149 & 15 & 30 \\
\hline L-Phenylalanine & 165 & 15 & 66 \\
\hline L-Proline & 115 & 20 & 40 \\
\hline L-Serine & 105 & 30 & - \\
\hline L-Threonine & 119 & 20 & 95 \\
\hline L-Tryptophan & 204 & 5 & 16 \\
\hline L-Tyrosine & 181 & 20 & 104.2 \\
\hline L-Valine & 117 & 20 & - \\
\hline \multicolumn{4}{|l|}{ Vitamins } \\
\hline Biotin & 244 & 0.2 & 0.013 \\
\hline Choline chloride & 140 & 3 & 4 \\
\hline D-Calcium pantothenate & 477 & 0.25 & 4 \\
\hline Folic acid & 441 & 1 & 4 \\
\hline Niacinamide & 122 & 1 & 4 \\
\hline p-Aminobenzoic acid & 137 & 1 & - \\
\hline Pyridoxine hydrochloride & 206 & 1 & 4 \\
\hline Riboflavin & 376 & 0.2 & 0.4 \\
\hline Thiamine hydrochloride & 337 & 1 & 4 \\
\hline Vitamin B12 & 1355 & 0.005 & 0.013 \\
\hline i-Inositol & 180 & 35 & - \\
\hline myo-Inositol & 180 & - & 7.2 \\
\hline \multicolumn{4}{|l|}{ Inorganic salts } \\
\hline Calcium nitrate & 236 & 100 & - \\
\hline Calcium chloride dihydrate & 147 & - & 218.6 \\
\hline Magnesium sulfate & 246 & 100 & 200 \\
\hline Potassium chloride & 75 & 400 & 330 \\
\hline Potassium nitrate & 101 & - & 0.076 \\
\hline Sodium bicarbonate & 84 & 2000 & 3025 \\
\hline
\end{tabular}


Sodium chloride

Sodium phosphate dibasic anhydrous

Sodium pyruvate

Sodium selenite

Other Components

D-Glucose (Dextrose)

Glutathione (reduced)

Phenol Red

HEPES

\section{8}

142

110

263

180

307

376.4

238
6000

800

$-$

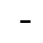

2000

1

5

$-$
4505

125

110

0.0173

4500

15

5958

$\underline{\text { Reagents and solutions for isometric force measurements }}$

\begin{tabular}{|c|c|c|c|c|}
\hline Stock solutions & Molar mass & Mass & Volume of water & Final concentration \\
\hline $\mathrm{CaCl}_{2}$ & $147.02 \mathrm{~g} / \mathrm{mol}$ & $165.57 \mathrm{~g}$ & $500 \mathrm{~mL}$ & $2.25 \mathrm{~mol} / \mathrm{L}$ \\
\hline $\mathrm{MgCl}_{2}$ & $203.01 \mathrm{~g} / \mathrm{mol}$ & $106.83 \mathrm{~g}$ & $500 \mathrm{~mL}$ & $1.05 \mathrm{~mol} / \mathrm{L}$ \\
\hline
\end{tabular}

Three separate stocks are prepared and stored at $4{ }^{\circ} \mathrm{C}$ to be used for the preparation of the working Tyrode's solution.

$\underline{\text { Stock } 1}$

Tyrode's Calcium

$0.2 \mathrm{mmol} / \mathrm{L}$

$\mathrm{NaCl}$

$58.44 \mathrm{~g} / \mathrm{mol}$

$175 \mathrm{~g}$

$\mathrm{KCl}$

$74.56 \mathrm{~g} / \mathrm{mol}$

$10 \mathrm{~g}$

$\mathrm{CaCl}_{2}$ stock

$2.25 \mathrm{~mol} / \mathrm{L}$

$2.22 \mathrm{~mL}$

$\mathrm{MgCl}_{2}$ stock

$1.05 \mathrm{~mol} / \mathrm{L}$

$25 \mathrm{~mL}$

Adjusted with ddH2O

$1000 \mathrm{~mL}$

$\underline{\text { Stock II }}$

$\mathrm{NaHCO}_{3}$

$84.01 \mathrm{~g} / \mathrm{mol} \quad 50 \mathrm{~g}$

$\mathrm{ddH}_{2} \mathrm{O}$

$18 \mathrm{~g} / \mathrm{mol}$

$1000 \mathrm{~mL}$

$\underline{\text { Stock III }}$

$\mathrm{NaH}_{2} \mathrm{PO}_{4}$

$137.99 \mathrm{~g} / \mathrm{mol} \quad 5.8 \mathrm{~g}$

$\mathrm{ddH}_{2} \mathrm{O}$

$18 \mathrm{~g} / \mathrm{mol}$

$1000 \mathrm{~mL}$ 
Tyrode's working solution

\begin{tabular}{|l|c|c|}
\hline Stock I & $80 \mathrm{~mL}$ & $200 \mathrm{~mL}$ \\
\hline Stock II & $76 \mathrm{~mL}$ & $190 \mathrm{~mL}$ \\
\hline Stock III & $20 \mathrm{~mL}$ & $50 \mathrm{~mL}$ \\
\hline Glucose & $2 \mathrm{~g}$ & $5 \mathrm{~g}$ \\
\hline Ascorbic acid & $100 \mathrm{mg}$ & $0.5 \mathrm{mg}$ \\
\hline Adjust with $\mathrm{ddH}_{2} \mathrm{O}$ & $2 \mathrm{~L}$ & $5 \mathrm{~L}$ \\
\hline
\end{tabular}

Tyrode's working solution is prepared as indicated above just prior to isometric force measurements. It is important to note that $50 \%$ of the final $\mathrm{ddH}_{2} \mathrm{O}$ should be added first to prevent precipitations in the stock I-III (e.g. $\mathrm{CaHPO}_{4}$ or $\mathrm{MgHPO}_{4}$ ). 
$\underline{\text { Reagents for polyacrylamide gel electrophoresis (PAGE) }}$

\begin{tabular}{|c|c|c|c|}
\hline Buffer & Constituent & Company & Amount \\
\hline \multirow{6}{*}{ Laemmli buffer } & Tris $\mathrm{HCl}, \mathrm{pH} 6.8$ & Carl Roth & $2 \mathrm{~mL}$ \\
\hline & Bromophenol Blue $0.5 \%$ & AppliChem & $0.5 \mathrm{~mL}$ \\
\hline & SDS & AppliChem & $0.8 \mathrm{~g}$ \\
\hline & Glycerin $100 \%$ & AppliChem & $1 \mathrm{~mL}$ \\
\hline & $\beta$-Mercaptoethanol & AppliChem & $1 \mathrm{~mL}$ \\
\hline & Adjusted with $\mathrm{ddH}_{2} \mathrm{O}$ & & $10 \mathrm{~mL}$ \\
\hline \multirow{6}{*}{ Separating gel $(10 \%, 2 x)$} & $\mathrm{ddH}_{2} \mathrm{O}$ & & $4 \mathrm{~mL}$ \\
\hline & Tris $\mathrm{HCl}(1.5 \mathrm{~mol} / \mathrm{L}) \mathrm{pH} 8.8$ & Carl Roth & $2.5 \mathrm{~mL}$ \\
\hline & Acrylamide $30 \%$ & AppliChem & $3.34 \mathrm{~mL}$ \\
\hline & SDS $10 \%$ & AppliChem & $0.1 \mathrm{~mL}$ \\
\hline & Ammonium phosphate $10 \%$ & Life technologies & $0.1 \mathrm{~mL}$ \\
\hline & TEMED & AppliChem & $0.01 \mathrm{~mL}$ \\
\hline \multirow{6}{*}{ Collecting gel $(6 \%, 2 x)$} & $\mathrm{ddH}_{2} \mathrm{O}$ & & $3.12 \mathrm{~mL}$ \\
\hline & Tris $\mathrm{HCl}(0.5 \mathrm{~mol} / \mathrm{L}) \mathrm{pH} 6.8$ & Carl Roth & $1.5 \mathrm{~mL}$ \\
\hline & Acrylamide $30 \%$ & AppliChem & $1.2 \mathrm{~mL}$ \\
\hline & SDS $10 \%$ & AppliChem & $0.06 \mathrm{~mL}$ \\
\hline & Ammonium phosphate $10 \%$ & Life technologies & $0.06 \mathrm{~mL}$ \\
\hline & TEMED & AppliChem & $0.006 \mathrm{~mL}$ \\
\hline \multirow{4}{*}{$\begin{array}{l}\text { Running buffer (10x) } \\
\text { (diluted to } 1 \mathrm{x} \text { before use) }\end{array}$} & Tris base & Carl Roth & $30.3 \mathrm{~g}$ \\
\hline & Glycine & AppliChem & $144 \mathrm{~g}$ \\
\hline & SDS & AppliChem & $10 \mathrm{~g}$ \\
\hline & $\mathrm{ddH}_{2} \mathrm{O}$ & & $1 \mathrm{~L}$ \\
\hline
\end{tabular}


$\underline{\text { Western Blot }}$

\begin{tabular}{|c|c|c|c|}
\hline Buffer & Constituent & Company & Amount \\
\hline \multirow{4}{*}{ Transfer Buffer (10x) } & Tris base & Carl Roth & $75.6 \mathrm{~g}$ \\
\cline { 2 - 4 } & Glycine & AppliChem & $74.4 \mathrm{~g}$ \\
\cline { 2 - 4 } & SDS & AppliChem & $15 \mathrm{~g}$ \\
\cline { 2 - 4 } & Adjust with ddH $\mathrm{H}_{2} \mathrm{O}$ & & $1 \mathrm{~L}$ \\
\hline \multirow{2}{*}{$\begin{array}{c}\text { Transfer buffer with } \\
\text { Methanol }\end{array}$} & Transfer buffer $(10 \mathrm{x})$ & & $10 \mathrm{~mL}$ \\
\cline { 2 - 4 } & Methanol & Carl Roth & $20 \mathrm{~mL}$ \\
\cline { 2 - 4 } & Adjusted with $\mathrm{ddH}_{2} \mathrm{O}$ & & $100 \mathrm{~mL}$ \\
\hline \multirow{3}{*}{ TBST } & Tris $\mathrm{HCl}(1 \mathrm{~mol} / \mathrm{L}) \mathrm{pH} 7.6$ & Carl Roth & $20 \mathrm{~mL}$ \\
\cline { 2 - 4 } & NaCl (5 mol/L) & Carl Roth & $33 \mathrm{~mL}$ \\
\cline { 2 - 4 } & Tween-20 & Sigma-Aldrich & $1 \mathrm{~mL}$ \\
\cline { 2 - 4 } & Adjusted with ddH $\mathrm{H}_{2} \mathrm{O}$ & & $1 \mathrm{~L}$ \\
\hline \multirow{2}{*}{\begin{tabular}{c} 
Blocking solution \\
\cline { 2 - 4 }
\end{tabular}} & BSA & Sigma Aldrich & $5 \mathrm{~g}$ \\
\cline { 2 - 4 } & TBST & & $100 \mathrm{~mL}$ \\
\hline
\end{tabular}


$\underline{\text { RT-PCR and qPCR }}$

Table 1: Primers used in this study

\begin{tabular}{|c|c|c|c|c|c|}
\hline Gene & $\mathbf{F}$ & $\mathbf{R}$ & Purpose & $\begin{array}{c}\text { Size } \\
\text { (bp) }\end{array}$ & Acc \# \\
\hline OCT4 & $\begin{array}{c}\text { CAGTGCCCGAAACCC } \\
\text { ACAC }\end{array}$ & $\begin{array}{c}\text { GGAGACCCAGCAGC } \\
\text { CTCAAA }\end{array}$ & qPCR & 161 & $\begin{array}{c}\text { NM_002701 } \\
\text { NM_203289 } \\
\text { NM_001173531 }\end{array}$ \\
\hline NKX2-5 & $\begin{array}{c}\text { ACAACTTCGTGAACT } \\
\text { TCGGCG }\end{array}$ & $\begin{array}{c}\text { GTGGACACTCCCGAG } \\
\text { TTGCTCT }\end{array}$ & qPCR & 82 & $\begin{array}{c}\text { NM_001166175.1 } \\
\text { NM_001166176.1 } \\
\text { NM_004387.3 }\end{array}$ \\
\hline ISL1 & $\begin{array}{c}\text { CGCCTTGCAGAGTGA } \\
\text { CATAG }\end{array}$ & $\begin{array}{c}\text { GGACTGGCTACCATG } \\
\text { CTGTT }\end{array}$ & qPCR & 147 & NM_002202.2 \\
\hline MESP-1 & $\begin{array}{c}\text { TGTGAGCACCGAGG } \\
\text { CTTTT }\end{array}$ & $\begin{array}{c}\text { CTCCTGCTTGCCTCA } \\
\text { AAGTG }\end{array}$ & qPCR & 82 & NM_018670.3 \\
\hline $\begin{array}{c}\text { MYL2 } \\
\text { MLC2v) }\end{array}$ & $\begin{array}{c}\text { GGCGCCAACTCCAAC } \\
\text { GTGTT }\end{array}$ & $\begin{array}{c}\text { ACGTTCACTCGCCCA } \\
\text { AGGGC }\end{array}$ & qPCR & 149 & NM_000432.2 \\
\hline GAPDH & $\begin{array}{c}\text { CCTAAGATCATCAGC } \\
\text { AATGCC }\end{array}$ & $\begin{array}{c}\text { ATGTTCTGGAGAGCC } \\
\text { CCGC }\end{array}$ & qPCR & 189 & NM_002046.3 \\
\hline
\end{tabular}

Table 2: Conditions for $\mathrm{qPCR}$ reaction

\begin{tabular}{|c|c|c|}
\cline { 2 - 3 } \multicolumn{1}{c|}{} & For 1x reaction & Final Concentration \\
\hline DEPC water & $3.9 \mu \mathrm{L}$ & NA \\
\hline 2x Syber Green & $5 \mu \mathrm{L}$ & NA \\
\hline Primer (F) & $0.05 \mu \mathrm{L}$ & $50 \mathrm{nmol} / \mathrm{L}$ \\
\hline Primer (R) & $0.05 \mu \mathrm{L}$ & $50 \mathrm{nmol} / \mathrm{L}$ \\
\hline cDNA & $1 \mu \mathrm{L}$ & NA \\
\hline Working volume/well & $10 \mu \mathrm{L}$ & NA \\
\hline
\end{tabular}


Table 3: qPCR program used

\begin{tabular}{|c|c|c|c|}
\hline Cycle Step & Temperature & Time & Cycles \\
\hline & $50^{\circ} \mathrm{C}$ & 2 minutes & $1 \mathrm{X}$ \\
\hline Initial Denaturation & $95^{\circ} \mathrm{C}$ & 10 minutes & \multirow{2}{*}{$40 \mathrm{X}$} \\
\hline Denaturation & $95^{\circ} \mathrm{C}$ & 15 seconds & 1 minute \\
\hline Annealing & $60^{\circ} \mathrm{C}$ & 15 seconds & \multirow{2}{*}{$1 \mathrm{X}$} \\
\hline Extension & $95^{\circ} \mathrm{C}$ & 15 seconds & \\
\hline Final extension & $60^{\circ} \mathrm{C}$ & 15 seconds & \\
\hline Inactivation & $95^{\circ} \mathrm{C}$ & & \\
\hline
\end{tabular}


Table 4: Antibodies used in this study

(FC-Flow cytometry, IF-Immunofluorescence, WB-Western blot)

\begin{tabular}{|c|c|c|c|c|}
\hline Antibody/Stain & Company & Cat No. & Marker & $\begin{array}{l}\text { Dilution } \\
\text { Factor }\end{array}$ \\
\hline $\operatorname{IgG}_{1}$ & RnD systems & MAB002 & Control & $\begin{array}{l}1: 100 \mathrm{FC} \\
1: 100 \mathrm{IF}\end{array}$ \\
\hline $\begin{array}{c}\alpha \text {-actinin (mouse } \\
\operatorname{IgG} \text { ) }\end{array}$ & Sigma & A7811 & Cardiomyocyte & $\begin{array}{l}\text { 1:4000 FC } \\
1: 1000 \mathrm{IF}\end{array}$ \\
\hline CD90 (mouse IgG) & RnD systems & MAB2067 & Stromal cell & $1: 500 \mathrm{FC}$ \\
\hline WT-1 (rabbit IgG) & Abcam & Ab89901 & Epicardial cell & $1: 200 \mathrm{IF}$ \\
\hline $\mathrm{TH}$ & Millipore & Ab152 & Neurons & $1: 1000 \mathrm{IF}$ \\
\hline GAPDH (mouse) & Zytomed systems & $607-0140$ & $\begin{array}{l}\text { GAPDH protein (37 } \\
\mathrm{kDa})\end{array}$ & $1: 50000 \mathrm{WB}$ \\
\hline SMAD1 (rabbit) & Cell Signaling & 9743 & $\begin{array}{c}\text { Total SMAD } \\
\text { protein }(60 \mathrm{kDa})\end{array}$ & 1:1000 WB \\
\hline $\begin{array}{c}\text { Phospho- } \\
\text { SMAD1/5/8 (rabbit) }\end{array}$ & Cell Signaling & 13820 & $\begin{array}{l}\text { Phosphorylated } \\
\text { SMAD1/5/8 (60 } \\
\text { kDa) }\end{array}$ & 1:1000 WB \\
\hline ERK1/2 (rabbit) & Cell Signaling & 9102 & $\begin{array}{c}\text { Total ERK1/2 } \\
\text { protein }(44 / 42 \mathrm{kDa})\end{array}$ & 1:1000 WB \\
\hline $\begin{array}{l}\text { Phospho-ERK1/2 } \\
\text { (rabbit) }\end{array}$ & Cell Signaling & 9101 & $\begin{array}{c}\text { Phosphorylated } \\
\text { ERK1/2 (44/42 } \\
\mathrm{kDa})\end{array}$ & 1:1000 WB \\
\hline $\begin{array}{c}\text { Goat anti-mouse } \\
\text { Alexafluor } 488\end{array}$ & Invitrogen & A-11001 & NA & $\begin{array}{c}1: 1000 \mathrm{FC} \\
1: 400 \mathrm{IF}\end{array}$ \\
\hline $\begin{array}{c}\text { Phalloidin } \\
\text { Alexafluor } 546 \\
\end{array}$ & Invitrogen & A22283 & NA & $1: 50 \mathrm{IF}$ \\
\hline Hoechst 33342 & Invitrogen & $\mathrm{H} 3570$ & NA & $\begin{array}{l}1: 1000 \mathrm{FC} \\
1: 1000 \mathrm{IF}\end{array}$ \\
\hline $\begin{array}{l}\text { Anti-mouse-IgG } \\
\text { HRP conjugated }\end{array}$ & Dako & P 0161 & NA & $1: 10000 \mathrm{WB}$ \\
\hline $\begin{array}{l}\text { Anti-rabbit IgG } \\
\text { HRP conjugated }\end{array}$ & Dako & 0448 & NA & 1:5000 WB \\
\hline
\end{tabular}


Chemical structure of 15 newly-synthesized chalcones (kindly provided by Prof. Dr. Dr. L. F. Tietze at the Institute of Organic and Biomolecular Chemistry, Georg-August-Universität Göttingen):<smiles>COc1ccc(C(=O)/C=C/c2ccc(F)cc2)cc1</smiles>

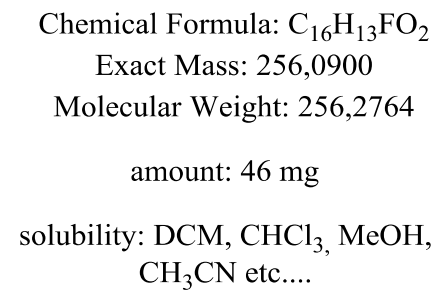<smiles>COc1ccc(/C=C/C(=O)c2ccc(OC)cc2)cc1</smiles>

Chemical Formula: $\mathrm{C}_{17} \mathrm{H}_{16} \mathrm{O}_{3}$ Exact Mass: 268,1099

Molecular Weight: 268,3120 amount: $43 \mathrm{mg}$

solubility: $\mathrm{DCM}, \mathrm{CHCl}_{3}, \mathrm{MeOH}$, $\mathrm{CH}_{3} \mathrm{CN}$ etc....<smiles>COc1ccc(C(=O)/C=C/c2ccc(NC(C)=O)cc2)cc1</smiles><smiles>Cc1ccc(C(=O)/C=C/c2ccc(F)cc2)cc1</smiles>

Chemical Formula: $\mathrm{C}_{16} \mathrm{H}_{13} \mathrm{FO}$ Exact Mass: 240,0950

Molecular Weight: 240,2774 amount: $30 \mathrm{mg}$ solubility: DCM, $\mathrm{CHCl}_{3}, \mathrm{MeOH}$, $\mathrm{CH}_{3} \mathrm{CN}$ etc....<smiles>COc1ccc(C(=O)/C=C/c2ccc(C(=O)OCC(C)(C)C)cc2)cc1</smiles> 
<smiles>COc1ccc(/C=C/C(=O)c2ccc([N+](=O)[O-])cc2)cc1</smiles><smiles>CC(=O)c1ccc(/C=C/C(=O)c2ccc(F)cc2)cc1</smiles>

Chemical Formula: $\mathrm{C}_{16} \mathrm{H}_{13} \mathrm{NO}_{4}$ Exact Mass: 283,0845

Molecular Weight: 283,2830 amount: $21 \mathrm{mg}$ solubility: $\mathrm{DCM}, \mathrm{CHCl}_{3}, \mathrm{MeOH}$, $\mathrm{CH}_{3} \mathrm{CN}$ etc....

Chemical Formula: $\mathrm{C}_{17} \mathrm{H}_{13} \mathrm{FO}_{3}$ Exact Mass: 284,0849

Molecular Weight: 284,2864 amount: $35 \mathrm{mg}$ solubility: DCM, $\mathrm{CHCl}_{3}, \mathrm{MeOH}$, $\mathrm{CH}_{3} \mathrm{CN}$ etc....

Chemical Formula: $\mathrm{C}_{15} \mathrm{H}_{10} \mathrm{FNO}_{3}$ Exact Mass: 271,0645

Molecular Weight: 271,2474 amount: $17 \mathrm{mg}$ solubility: $\mathrm{DCM}, \mathrm{CHCl}_{3}, \mathrm{MeOH}$, $\mathrm{CH}_{3} \mathrm{CN}$ etc....

Chemical Formula: $\mathrm{C}_{16} \mathrm{H}_{14} \mathrm{O}_{3}$ Exact Mass: 254,0943

Molecular Weight: 254,2850 amount: $23 \mathrm{mg}$ solubility: DCM, $\mathrm{CHCl}_{3}, \mathrm{MeOH}$, $\mathrm{CH}_{3} \mathrm{CN}$ etc....<smiles>CC(=O)Nc1ccc(/C=C/C(=O)c2ccc(O)cc2)cc1</smiles>

Chemical Formula: $\mathrm{C}_{17} \mathrm{H}_{15} \mathrm{NO}_{3}$ Exact Mass: 281,1052 Molecular Weight: 281,3110 amount: $9 \mathrm{mg}$ solubility: DMSO, $\mathrm{CH}_{3} \mathrm{CN}$ 
<smiles>[R16][13CH2]/C=C/c1ccc(O)cc1</smiles><smiles>[R16][13CH2]c1ccc(C)cc1</smiles><smiles>COc1ccc(C(=O)/C=C/c2cc(F)ccc2Br)cc1</smiles><smiles>COc1cccc(/C=C/C(=O)c2ccc(F)cc2)c1</smiles>

15.<smiles></smiles>

Chemical Formula: $\mathrm{C}_{15} \mathrm{H}_{11} \mathrm{NO}_{4}$ Exact Mass: 269,0688

Molecular Weight: 269,2560 amount: $9 \mathrm{mg}$ solubility: $\mathrm{DCM}, \mathrm{CHCl}_{3}, \mathrm{MeOH}$, $\mathrm{CH}_{3} \mathrm{CN}$ etc....

Chemical Formula: $\mathrm{C}_{16} \mathrm{H}_{13} \mathrm{NO}_{3}$ Exact Mass: 267,0895

Molecular Weight: 267,2840 amount: $33 \mathrm{mg}$ solubility: $\mathrm{DCM}, \mathrm{CHCl}_{3}, \mathrm{MeOH}$, $\mathrm{CH}_{3} \mathrm{CN}$ etc....

Chemical Formula: $\mathrm{C}_{16} \mathrm{H}_{12} \mathrm{BrFO}_{2}$ Exact Mass: 334,0005

Molecular Weight: 335,1724 amount: $59 \mathrm{mg}$ solubility: $\mathrm{DCM}, \mathrm{CHCl}_{3}, \mathrm{MeOH}$, $\mathrm{CH}_{3} \mathrm{CN}$ etc....

\footnotetext{
Chemical Formula: $\mathrm{C}_{16} \mathrm{H}_{13} \mathrm{FO}_{2}$ Exact Mass: 256,0900 Molecular Weight: 256,2764 amount: $50 \mathrm{mg}$ solubility: DCM, $\mathrm{CHCl}_{3}, \mathrm{MeOH}$, $\mathrm{CH}_{3} \mathrm{CN}$ etc....
}

\footnotetext{
Chemical Formula: $\mathrm{C}_{15} \mathrm{H}_{11} \mathrm{FO}_{2}$ Exact Mass: 242,0743

Molecular Weight: 242,2494

amount: $32 \mathrm{mg}$ solubility: DCM, $\mathrm{CHCl}_{3}, \mathrm{MeOH}$, $\mathrm{CH}_{3} \mathrm{CN}$ etc....
} 


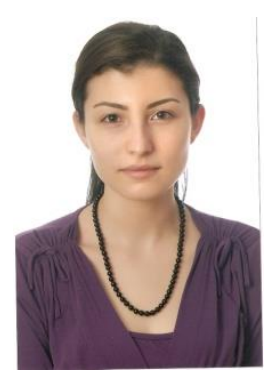

\title{
Curriculum Vitae
}

\author{
Farah S. Raad
}

\section{Personal Information}

\author{
Name: $\quad$ Farah S. Raad \\ Nationality: Lebanese \\ Gender: $\quad$ Female \\ Date of Birth: October $9^{\text {th }}, 1988$ \\ Address: University Medical Center Göttingen \\ Georg-August Universität Göttingen \\ Robert-Koch str.40 \\ 37075 Göttingen \\ Deutschland \\ Phone \#: $\quad 004915736369981$ \\ Email: $\quad$ farah.raad@med.uni-goettingen.de
}

\section{$\underline{\text { Education }}$}

October 2011 - present

September 2009-August2011

September 2006-June 2009
Georg-August Universität Göttingen, Department of Pharmacology, Heart Research Center Göttingen (HRCG), University Medical Center Göttingen. Göttingen Germany

Pursuing PhD in Molecular Medicine, Expected Date of Graduation: June 2016

Jacobs University Bremen, Bremen Germany M. Sc. in Nanomolecular Science (Chemistry)

American University of Beirut, Beirut Lebanon B.S in Chemistry 


\section{$\underline{\text { Research Experience }}$}

July 2009-September 2009

October 2007- June 2009

\section{University of Windsor, Windsor Canada}

Intern in the Department of Chemistry

Project based on the synthesis and characterization of Liquid Cyrstalline structures

\section{American University of Beirut, Beirut Lebanon}

Undergraduate Research Assistant in the Department of

Chemistry

Project based on

- Synthesis and characterization of novel organic semiconductors.

- Experimental analysis of the fluorescence effect of different anions on the fluorescence and absorbance of various sensors.

- Synthesis and binding studies of novel organic sensors for anions.

\section{$\underline{\text { Skills }}$}

Chemistry-related skills:

- Separation, purification, cystallization, isolation and identification of organic and inorganic compounds.

- Titration by fluorescence and UV-Vis.

- Characterization using analytical techniques, e.g. NMR, IR, XRD, TGA.

Biology-related skills:

- Cell culture, molecular biology, cell biology, tissue engineering

- Characterization using: flow cytometry, immunohistochemistry, western blotting, qPCR

Computer skills: Good Knowledge of Microsoft Office, SciFinder, EndNote, Origin, Diamond, Graph Pad Prism, Adobe Illustrator

Language skills: Arabic (mother tongue)

English (fluent)

French and German (basic knowledge) 


\section{Publications}

1. Raad FS, Moustafa RM, Jradi FM, Al-Sayah MH, Kaafarani BR (2010) Synthesis and Anion-Binding Studies of A Novel Pyrene Discotic. Tetrahedron. 66:2944-2952.

2. Banerjee A, Raad FS, Vankova N, Bassil BS, Heine T, Kortz U (2011) Polyoxomolybdodiphosphonates: Examples Incorporating Ethylidenepyridines. Inorg. Chem. 50:11667.

3. Eichhorn SH, Chen S, Ahmida M, Demenev A, Kayal H, Raad FS, Kaafarani BR, Patwardhan S, Grozema FC, Siebbeles LDA, Taerum T, Perepichka DF, Klenkler R (2011) A combined study of mesomorphism, optical, and electronic properties of donoracceptor columnar liquid crystals. Proceedings of SPIE. 8114:811402\1.

4. Chen S, Raad FS, Ahmida M, Kaafarani BR, Eichhorn SH (2013) Columnar Mesomorphism of Fluorescent Broad-Shaped Quinoxalinophenanthrophenazine Derivatives with Donor-Acceptor Structure. Org. Lett. 15 (3):558-561.

5. Al-Kadamany G, Bassil BS, Raad FS, Kortz U (2014) The Oxalato-TitaniumContaining Tungstophosphate(V) Dimers, $\left[\mathrm{Ti}_{8}\left(\mathrm{C}_{2} \mathrm{O}_{4}\right)_{8} \mathrm{P}_{2} \mathrm{~W}_{18} \mathrm{O}_{76}\left(\mathrm{H}_{2} \mathrm{O}\right)_{4}\right]^{18-}$ and $\left[\mathrm{Ti}_{6}\left(\mathrm{C}_{2} \mathrm{O}_{4}\right)_{4} \mathrm{P}_{4} \mathrm{~W}_{32} \mathrm{O}_{124}\right]^{20-}$. Journal of Cluster Science. $25: 3$

6. El-Ballouli AO, Kayal H, Shuai C, Zeidan TA, Raad FS, Leng S, Wex B, Cheng S, Eichhorn SH, Kaafarani BR (2014) Lateral extension induces columnar mesomorphism in crucifix shaped quinoxalinophenanthrophenazines. Tetrahedron, 71:308-314.

7. Zeidler S, Meckbach C, Tacke R, Raad FS, Roa A, Uchida S, Zimmermann WH, Wingender E and Gueltas M (2016). Computational Detection of Stage-Specific Transcription Factor Clusters during Heart Development. Front. Genet. 7:33.

8. Hudson JE, Tiburcy M, Raad FS, Roa A, Muppala V, Döker S, Bartels I, Dressel R, Ziebolz D, Behr R, Zimmermann WH (2016) Directed Assembly of Human and NonHuman Primate Heart Muscle from Embryonic and Induced Pluripotent Stem Cells. In Revision.

9. Tiburcy M, Hudson JE, Balfanz P, Schlick S, Meyer T, Chang Liao ML, Levent E, Raad FS, Zeidler S, Wingender E, Riegler S, Wang M, Gold JD, Kehat I, Wettwer E, Ravens U, Dierickx P, Van Laake L, Goumans MJ, Toischer K, Hasenfuss G, Couture LA, Unger A, Linke W, Araki T, Neel B, Keller G, Gepstein L, Wu J and Zimmerman WH. (2016). Directed Maturation of Engineered Human Myocardium for Applications in Heart Failure Modelling and Repair. In Revision. 


\section{$\underline{\text { Abstracts }}$}

1. Raad FS, Jradi FM, Mustafa RM, Al-Sayah MH, Kaafarani BR (2008) Quinoxalino$\left[2^{\prime}, 3^{\prime}: 9,10\right]$ phenanthro[4,5-abc]phenazine Fluorescent Sensors for Cations and Anions. $4^{\text {th }}$ International Conference on Molecular Electronics (ElecMol08), Grenoble, France.

2. Raad FS, Jradi FM, Mustafa RM, Al-Sayah MH, Kaafarani BR (2009) Fluorescent and Colorimetric Organic Sensors for Cations and Anions. Taibah International Chemistry Conference, Al-Madinah, Saudi Arabia.

3. Kaafarani BR, Moustafa RM, Jradi FM, Degheili JA, Raad FS, El-Ballouli AO, Lucas L, Leng S, Zeidan TA, Wex B, Patra D, Al-Sayah MH, DeLongchamp DM, Jabbour GE, Cheng SZD (2009) Novel Pyrene-Based Discoids Exploited: Discovering Materials For Sensors, Electronic and Optoelectronic Devices. $238^{\text {th }}$ ACS National Meeting, Washington, DC, USA.

4. Raad FS, Roa A, Hudson JE, Zimmermann WH (2014) Altering Tissue Functionality Using Growth Factors and Small Molecules in a Bioengineered Heart Muscle. Basic Science Meeting-DGK, Dusseldörf, Germany.

5. Raad FS, Tiburcy M, Roa A, Zimmermann WH (2015) Simulating Human Myocardium in vitro. Fondation Leducq Meeting 2015, Trieste, Italy.

6. Raad FS, Roa A, Hudson JE, Tiburcy M, Uchida S, Zimmermann WH (2016) Modeling Human Heart Development and Faulty Cardiogenesis in Bioengineered Heart Muscle. DGK-HKK Mannheim 2016. Mannheim, Germany. 


\section{$\underline{\text { References }}$}

Prof. Dr. Bilal R. Kaafarani

Department of Chemistry

American University of Beirut

Beirut, Lebanon

E-mail: bilal.kaafarani@ aub.edu.lb

Prof. Dr. S. Holger Eichhorn

Department of Chemistry and Biochemistry

University of Windsor

Windsor, ON Canada

E-mail: eichhorn@uwindsor.ca

Prof. Dr. Ulrich Kortz

Professor of Chemistry

School of Engineering and Science

Jacobs University Bremen

Bremen, Germany

E-mail; u.kortz@jacobs-university.de

Prof. Dr. Wolfram-Hubertus Zimmermann

Department of Pharmacology and Toxicology

Heart Research Center Göttingen (HRCG)

University Medical Center Göttingen

Georg-August Universität Göttingen

Göttingen, Germany

E-mail: w.zimmermann@med.uni-goettingen.de 